ERNEST DRLANDQ LAWRENLE BERKELEY NATIGNAL LAB

\title{
Electron-Capture Delayed Fission Properties of Neutron-Deficient Einsteinium Nuclei
}

Dawn A. Shaughnessy

Nuclear Science Division

January 2000

Ph.D. Thesis 


\section{DISCLAIMER}

This document was prepared as an account of work sponsored by the United States Government. While this document is believed to contain correct information, neither the United States Government nor any agency thereof, nor The Regents of the University of California, nor any of their employees, makes any warranty, express or implied, or assumes any legal responsibility for the accuracy, completeness, or usefulness of any information, apparatus, product, or process disclosed, or represents that its use would not infringe privately owned rights. Reference herein to any specific commercial product, process, or service by its trade name, trademark, manufacturer, or otherwise, does not necessarily constitute or imply its endorsement, recommendation, or favoring by the United States Government or any agency thereof, or The Regents of the University of California. The views and opinions of authors expressed herein do not necessarily state or reflect those of the United States Government or any agency thereof, or The Regents of the University of California.

Ernest Orlando Lawrence Berkeley National Laboratory is an equal opportunity employer. 


\section{DISCLAIMER}

Portions of this document may be illegible in electronic image products. Images are produced from the best available original document. 


\title{
Electron-Capture Delayed Fission Properties of Neutron-Deficient Einsteinium Nuclei
}

\author{
Dawn A. Shaughnessy \\ Ph.D. Thesis \\ Department of Chemistry \\ University of California, Berkeley \\ and \\ Nuclear Science Division \\ Ernest Orlando Lawrence Berkeley National Laboratory \\ University of California \\ Berkeley, CA 94720
}

January 2000

This work was supported by the Director, Office of Science, of the U.S. Department of Energy under Contract No. DE-AC03-76SF00098. 


\title{
Electron-Capture Delayed Fission Properties of Neutron-Deficient Einsteinium Nuclei
}

\author{
Copyright 1999
}

by

\section{Dawn Angela Shaughnessy}

The U.S. Department of Energy has the right to use this document for any purpose whatsoever including the right to reproduce all or any part thereof. 


\title{
Abstract \\ Electron-Capture Delayed Fission Properties of Neutron-Deficient \\ Einsteinium Nuclei
}

by

\author{
Dawn Angela Shaughnessy \\ Doctor of Philosophy in Chemistry \\ University of California, Berkeley \\ Professor Darleane C. Hoffman, Chair
}

Electron-capture delayed fission (ECDF) properties of neutron-deficient einsteinium isotopes were investigated using a combination of chemical separations and on-line radiation detection methods. ${ }^{242} \mathrm{Es}$ was produced via the ${ }^{233} \mathrm{U}\left({ }^{14} \mathrm{~N}, 5 \mathrm{n}\right){ }^{242} \mathrm{Es}$ reaction at a beam energy of $87 \mathrm{MeV}$ (on target) in the lab system, and was found to decay with a half-life of $11 \pm 3$ seconds. The ECDF of ${ }^{242}$ Es showed a highly asymmetric mass distribution with an average pre-neutron emission total kinetic energy (TKE) of 183 $\pm 18 \mathrm{MeV}$. The probability of delayed fission $\left(\mathrm{P}_{\mathrm{DF}}\right)$ was measured to be $0.006 \pm 0.002$. In conjunction with this experiment, the excitation functions of the ${ }^{233} U\left({ }^{14} N, x n\right){ }^{247-x} E s$ and ${ }^{233} \mathrm{U}\left({ }^{15} \mathrm{~N}, \mathrm{xn}\right){ }^{248-\mathrm{x}}$ Es reactions were measured for ${ }^{243} \mathrm{Es},{ }^{244} \mathrm{Es}$ and ${ }^{245}$ Es at projectile energies between $80 \mathrm{MeV}$ and $100 \mathrm{MeV}$.

The ECDF of ${ }^{244}$ Es, produced via the ${ }^{237} \mathrm{~Np}\left({ }^{12} \mathrm{C}, 5 \mathrm{n}\right){ }^{244}$ Es reaction at an energy of $81 \mathrm{MeV}$ (on target) in the lab system, was also observed to have a highly asymmetric mass distribution with an average pre-neutron emission TKE of $186 \pm 19 \mathrm{MeV}$. The $\mathrm{P}_{\mathrm{DF}}$ was determined to be $(1.2 \pm 0.4) \times 10^{-4}$. The average TKE values measured for ${ }^{242}$ Es and 
${ }^{244}$ Es follow the trend observed in other ECDF systems where the TKE values are lower than for spontaneous fission.

${ }^{246} \mathrm{Es}$ and ${ }^{248}$ Es were both produced via the ${ }^{249} \mathrm{Cf}(\mathrm{p}, \mathrm{xn}){ }^{250-\mathrm{x}}$ Es reaction at energies of $37 \mathrm{MeV}$ and $18 \mathrm{MeV}$, respectively. Not enough fission events were recorded to determine the fission properties of these nuclides, but their $\mathrm{P}_{\mathrm{DF}}$ values were measured to be $(3.7 \pm 3.7) \times 10^{-5}$ and $(3.5 \pm 1.8) \times 10^{-6}$ for ${ }^{246}$ Es and ${ }^{248}$ Es, respectively. The $P_{D F}$ values for all four einsteinium isotopes show the same trend of exponentially increasing $P_{D F}$ with increasing electron-capture Q-value as previously observed.

The excitation function for the ${ }^{249} \mathrm{Cf}(\mathrm{p}, \mathrm{xn})^{250-\mathrm{x}}$ Es reaction was also studied at several different energies. The production cross sections of ${ }^{245} \mathrm{Es},{ }^{246} \mathrm{Es},{ }^{247} \mathrm{Es},{ }^{248} \mathrm{Es}$ and ${ }^{249} \mathrm{Es}$ were measured at proton energies between $21 \mathrm{MeV}$ and $40 \mathrm{MeV}$ and were consistent with previously reported values. 


\section{Dedication}

This dissertation is dedicated to my husband David for all of the love and support he has given me during my years in graduate school. Thank you for all of your help and encouragement and for just being the way you are. I would have never been able to do it without you. I will love you always and forever. 


\section{Table of Contents}

1. Introduction

$1.1 \quad$ Discovery of Einsteinium $\ldots \ldots \ldots \ldots \ldots \ldots \ldots \ldots \ldots \ldots \ldots \ldots \ldots$

1.2 Electron-Capture Delayed Fission $\ldots \ldots \ldots \ldots \ldots \ldots \ldots \ldots \ldots \ldots$

$1.3 \quad$ Isotope Production $\ldots \ldots \ldots \ldots \ldots \ldots \ldots \ldots \ldots \ldots \ldots \ldots \ldots$

2. Theory of Delayed Fission

2.1 Calculations of the Delayed Fission Probability ...............12

$2.2 \quad$ Requirements for $\mathrm{ECDF} \ldots \ldots \ldots \ldots \ldots \ldots \ldots \ldots \ldots \ldots \ldots \ldots \ldots \ldots$

3. Previous Work

3.1 Previous Delayed Fission Studies $\ldots \ldots \ldots \ldots \ldots \ldots \ldots \ldots \ldots .21$

3.2 Einsteinium Production and Excitation Functions $\ldots \ldots \ldots \ldots \ldots 23$

4. Targets and Irradiations

$4.1 \quad$ Target Configurations . . . . . . . . . . . . . . . . . . . . . 28

4.1.1 Single Target Bombardments . . . . . . . . . . . . . 28

4.1.2 Multiple Target Bombardments ................. 30

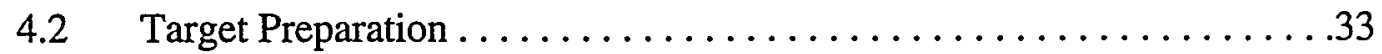

4.2.1 Uranium Targets $\ldots \ldots \ldots \ldots \ldots \ldots \ldots \ldots \ldots \ldots \ldots \ldots \ldots \ldots$

4.2.2 Neptunium Targets . . . . . . . . . . . . . . . . . . 36

4.2.3 Californium Targets ....................... 37

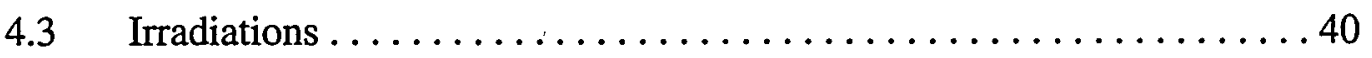


5. Experimental Procedures

$5.1 \quad$ On-Line Procedures .................................4 47

5.2 Chemical Separations and X-Ray/Fission Correlation Studies . . . . . . 54

$5.3 \quad$ Excitation Function Procedures ...................... 57

5.3.1 ${ }^{14,15} \mathrm{~N}+{ }^{233} \mathrm{U}$ Excitation Functions ................. 57

5.3.2 $\mathrm{p}+{ }^{249} \mathrm{Cf}$ Excitation Function . . . . . . . . . . . . . . 60

6. Results and Discussion

6.1 Electron-Capture Delayed Fission $\ldots \ldots \ldots \ldots \ldots \ldots \ldots \ldots \ldots, \ldots 3$

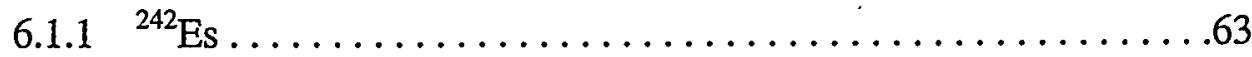

6.1.1.1 Fission Properties and Half-Life . ...........63

6.1.1.2 Search for $\alpha$-Decay in ${ }^{242}$ Es . ............. 71

6.1.1.3 $\mathrm{P}_{\mathrm{DF}}$ and $\log f t$ Values .................... 77

$6.1 .2{ }^{244}$ Es ..................................... 79

6.1.2.1 Initial Study Using the ${ }^{233} \mathrm{U}\left({ }^{15} \mathrm{~N}, 4 \mathrm{n}\right){ }^{244}$ Es Reaction . . . 79

6.1.2.2 ECDF Study Using the ${ }^{237} \mathrm{~Np}\left({ }^{12} \mathrm{C}, 5 \mathrm{n}\right){ }^{244}$ Es Reaction . . .82

6.1.2.2.1 Fission Properties ...............82

6.1.2.2.2 $\alpha$-Decay in ${ }^{244}$ Es . .............. 89

6.1.2.2.3 $\mathrm{P}_{\mathrm{DF}}$ and $\log f t$ Values ............. 93

$6.1 .3{ }^{246}$ Es and ${ }^{248}$ Es .............................. 95

6.1.3.1 $\alpha$-Decay .......................... 95

6.1.3.2 $\mathrm{P}_{\mathrm{DF}}$ and $\log f t$ Values $\ldots \ldots \ldots \ldots \ldots \ldots \ldots \ldots . .98$

6.1.3.3 X-ray/Fission Coincidence Measurements ......... 103

6.2 Einsteinium Excitation Functions $\ldots \ldots \ldots \ldots \ldots \ldots \ldots \ldots \ldots \ldots$ 
$6.2 .1{ }^{233} \mathrm{U}\left({ }^{14} \mathrm{~N}, \mathrm{xn}\right){ }^{247-\mathrm{x}}$ Es Reactions . . . . . . . . . . . . . . . 106

$6.2 .2{ }^{233} \mathrm{U}\left({ }^{15} \mathrm{~N}, \mathrm{xn}\right)^{248-\mathrm{x}}$ Es Reactions .......................114

$6.2 .3{ }^{249} \mathrm{Cf}(\mathrm{p}, \mathrm{xn})^{250-\mathrm{x}}$ Es Reactions . . . . . . . . . . . . . . 116

6.2.3.1 Efficiency of the LIM/Gas-Jet Transport System . . . . 116

6.2.3.1.1 Target Tests of the LIM System ..........116

6.2.3.1.2 Efficiency Measurement of the LIM System . 118

6.2.3.2 Effective Target Thickness ................122

6.2.3.3 MG Measurements .................. 125

6.2.3.4 Chemically Separated Samples .............. 134

6.2.3.5 Summary of Cross Sections ............... 141

6.2.4 The SPIT Code for Calculation of Cross Sections . . . . . . . . 148

6.2.4.1 The Model ...................... 148

6.2.4.2 Comparison With Experimental Data ........... 151

6.2.4.3 Another Example of a Cross Section Code ........ 156

\section{Summary}

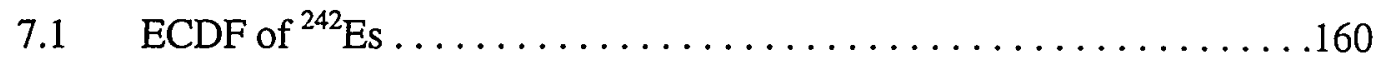

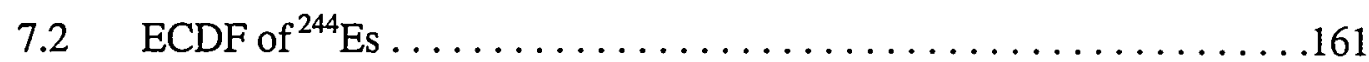

7.3 Fission Properties From ECDF Studies . ............... 162

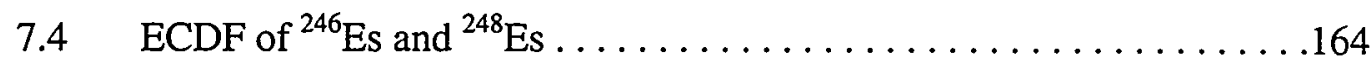

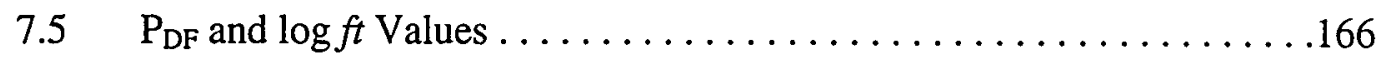

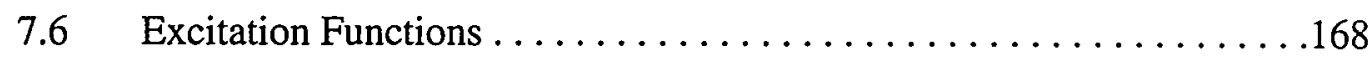

8. Conclusions and Future Research

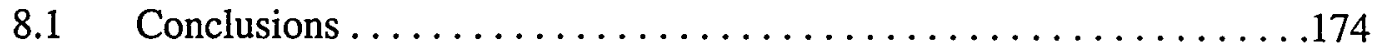


$8.2 \quad$ Future Research ............................. 176

References ............................... 179 


\section{List of Figures}

1.1 Two-dimensional drawing of the delayed fission process $\ldots \ldots \ldots \ldots \ldots$

1.2 Spontaneous fission half-lives $\ldots \ldots \ldots \ldots \ldots \ldots \ldots \ldots \ldots \ldots \ldots$

2.1 Portion of the table of isotopes showing nuclei likely to undergo ECDF . . . . 19

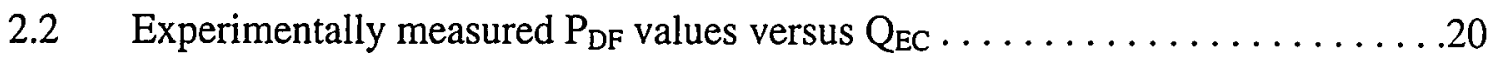

3.1 Excitation functions for the ${ }^{249} \mathrm{Cf}(\mathrm{p}, \mathrm{xn})^{250-\mathrm{x}}$ Es reactions $\ldots \ldots \ldots \ldots \ldots . \ldots 27$

4.1 Illustration of the single target recoil chamber $\ldots \ldots \ldots \ldots \ldots \ldots \ldots \ldots$

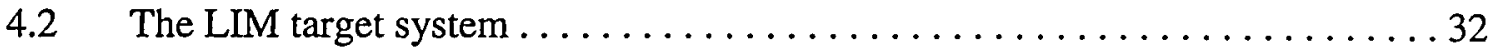

4.3 Schematic of the electroplating cell used for target production . . . . . . . 35

4.4 Flow chart of the HDEHP extraction procedure $\ldots \ldots \ldots \ldots \ldots \ldots \ldots$

4.5 Predicted cross sections for the ${ }^{233} U\left({ }^{14} \mathrm{~N}, \mathrm{xn}\right){ }^{247-\mathrm{x}}$ Es reactions $\ldots \ldots \ldots \ldots . .43$

4.6 Predicted cross sections for the ${ }^{233} U\left({ }^{15} N, x n\right)^{248-x}$ Es reactions . . . . . . . . 44

4.7 Predicted cross sections for the ${ }^{237} \mathrm{~Np}\left({ }^{12} \mathrm{C}, \mathrm{xn}\right){ }^{239-\mathrm{x}}$ Es reactions $\ldots \ldots \ldots \ldots 45$

4.8 Predicted cross sections for the ${ }^{249} \mathrm{Cf}(\mathrm{p}, \mathrm{xn}){ }^{250-\mathrm{x}} \mathrm{Es}$ reactions $\ldots \ldots \ldots \ldots \ldots 6$

5.1 Top view of the $\mathrm{MG}$ rotating wheel detection system $\ldots \ldots \ldots \ldots \ldots \ldots$

5.2 Cross-sectional view of the $\mathrm{MG}$ detector stations $\ldots \ldots \ldots \ldots \ldots \ldots \ldots$

$5.3 \quad \mathrm{MG} \alpha$-energy calibration using $\mathrm{a}^{212} \mathrm{~Pb}$ source $\ldots \ldots \ldots \ldots \ldots \ldots \ldots \ldots \ldots \ldots \ldots \ldots$

$5.4 \quad \mathrm{MG}$ fission energy calibration using a ${ }^{252} \mathrm{Cf}$ source $\ldots \ldots \ldots \ldots \ldots \ldots$

$5.5 \mathrm{X}$-ray/fission coincidence measurement setup $\ldots \ldots \ldots \ldots \ldots \ldots \ldots$

$5.6 \quad \mathrm{X}$-ray energy calibration using a ${ }^{249} \mathrm{Cf}$ source $\ldots \ldots \ldots \ldots \ldots \ldots$ 
6.1 Decay of coincident fission events from the ECDF of ${ }^{242}$ Es . . . . . . . . 64

6.2 Pre-neutron emission mass-yield distribution for the ECDF of ${ }^{242}$ Es . . . . . 66

6.3 Pre-neutron emission TKE distribution for the ECDF of ${ }^{242}$ Es . . . . . . . 68

6.4 Summed $\alpha$-spectrum from the ${ }^{233} \mathrm{U}\left({ }^{14} \mathrm{~N}, \mathrm{xn}\right){ }^{247-\mathrm{x}}$ Es reactions at $87 \mathrm{MeV} \ldots \ldots .73$

6.5 Summed $\alpha$-spectrum from the ${ }^{233} \mathrm{U}\left({ }^{14} \mathrm{~N}, \mathrm{xn}\right){ }^{247-\mathrm{x}}$ Es reactions at $87 \mathrm{MeV}$ (no

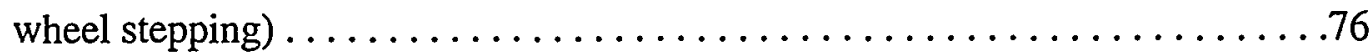

6.6 Summed $\alpha$-spectrum from the ${ }^{233} \mathrm{U}\left({ }^{15} \mathrm{~N}, \mathrm{xn}\right){ }^{248-\mathrm{x}}$ Es reactions at $86 \mathrm{MeV} \ldots \ldots .81$

6.7 Decay of coincident fission events from the ECDF of ${ }^{244} \mathrm{Es} \ldots \ldots \ldots \ldots . \ldots 85$

6.8 Pre-neutron emission mass-yield distribution for the ECDF of ${ }^{244}$ Es . . . . . .86

6.9 Pre-neutron emission TKE distribution for the ECDF of ${ }^{244}$ Es . . . . . . . . 87

6.10 Summed $\alpha$-spectrum from the ${ }^{237} \mathrm{~Np}\left({ }^{12} \mathrm{C}, \mathrm{xn}\right){ }^{249-\mathrm{x}}$ Es reactions at $81 \mathrm{MeV} \ldots \ldots 90$

6.11 Summed $\alpha$-spectrum from the ${ }^{237} \mathrm{~Np}\left({ }^{12} \mathrm{C}, \mathrm{xn}\right){ }^{249-\mathrm{x}}$ Es reactions at $81 \mathrm{MeV}$ (no

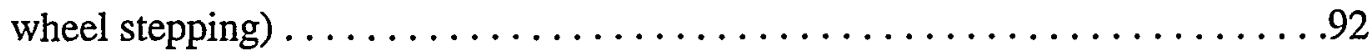

$6.12 \alpha$-spectrum from the ${ }^{249} \mathrm{Cf}(\mathrm{p}, \mathrm{xn})^{250-\mathrm{x}}$ Es reactions at $37 \mathrm{MeV} \ldots \ldots \ldots \ldots . .97$

$6.13 \alpha$-spectrum from the ${ }^{249} \mathrm{Cf}(\mathrm{p}, \mathrm{xn})^{250-\mathrm{x}}$ Es reactions at $18 \mathrm{MeV} \ldots \ldots \ldots \ldots . . .99$

$6.14 \mathrm{X}$-ray and $\alpha$-spectrum from the $\mathrm{x}$-ray/fission coincidence setup . . . . . . . 104

6.15 Decay of 7.9-MeV $\alpha$-particles from ${ }^{233} \mathrm{U}\left({ }^{14} \mathrm{~N}, \mathrm{xn}\right){ }^{247-\mathrm{x}}$ Es reactions at $80 \mathrm{MeV} .110$

6.16 Decay of 7.9-MeV $\alpha$-particles from ${ }^{233} \mathrm{U}\left({ }^{14} \mathrm{~N}, \mathrm{xn}\right){ }^{247-\mathrm{x}}$ Es reactions at $87 \mathrm{MeV} .111$

6.17 Experimentally determined cross sections for the ${ }^{233} \mathrm{U}\left({ }^{14} \mathrm{~N}, 4 \mathrm{n}\right){ }^{243}$ Es reaction . .113

$6.18 \alpha$-spectrum from the ${ }^{233} \mathrm{U}\left({ }^{15} \mathrm{~N}, \mathrm{xn}\right)^{248-\mathrm{x}} \mathrm{Es}$ reactions at $80 \mathrm{MeV} \ldots \ldots \ldots \ldots 115$

6.19 Estimation of recoil ranges for the ${ }^{250}$ Es compound nucleus in uranium . . . . 124

$6.20 \alpha$-spectrum from the ${ }^{249} \mathrm{Cf}(\mathrm{p}, \mathrm{xn})^{250-\mathrm{x}}$ Es reactions at $40 \mathrm{MeV} \ldots \ldots \ldots \ldots . .127$ 
6.21 Decay of 7.32-MeV and 7.73-MeV $\alpha$-particles from ${ }^{249} \mathrm{Cf}(\mathrm{p}, \mathrm{xn})^{250-\mathrm{x}} \mathrm{Es}$ reactions at $40 \mathrm{MeV}$

6.22 Decay of 7.3-MeV $\alpha$-particles from the ${ }^{249} \mathrm{Cf}(\mathrm{p}, \mathrm{xn})^{250-\mathrm{x}}$ Es reactions at $35 \mathrm{MeV}$

6.23 Decay of 6.75-MeV $\alpha$-particles from the ${ }^{249} \mathrm{Cf}(\mathrm{p}, \mathrm{xn})^{250-\mathrm{x}} \mathrm{Es}$ reactions at $40 \mathrm{MeV}$

6.24 Decay of 6.7-MeV $\alpha$-particles from the ${ }^{249} \mathrm{Cf}(\mathrm{p}, \mathrm{xn}){ }^{250-\mathrm{x}}$ Es reactions at

$21 \mathrm{MeV}$ and $28 \mathrm{MeV}$

6.25 Decay of 6.87-MeV $\alpha$-particles from the ${ }^{249} \mathrm{Cf}(\mathrm{p}, \mathrm{xn})^{250-\mathrm{x}} \mathrm{Es}$ reactions at $40 \mathrm{MeV}$ 140

6.26 Experimentally determined cross sections for the ${ }^{249} \mathrm{Cf}(\mathrm{p}, 5 \mathrm{n})^{245}$ Es reaction . . 142

6.27 Experimentally determined cross sections for the ${ }^{249} \mathrm{Cf}(\mathrm{p}, 4 \mathrm{n}){ }^{246}$ Es reaction . . 143

6.28 Experimentally determined cross sections for the ${ }^{249} \mathrm{Cf}(\mathrm{p}, 3 \mathrm{n})^{247}$ Es reaction . . 144

6.29 Experimentally determined cross sections for the ${ }^{249} \mathrm{Cf}(\mathrm{p}, 2 \mathrm{n}){ }^{248}$ Es reaction . . 145

6.30 Experimentally determined cross sections for the ${ }^{249} \mathrm{Cf}(\mathrm{p}, 1 \mathrm{n})^{249}$ Es reaction . . 146

6.31 Experimentally determined cross sections for the ${ }^{249} \mathrm{Cf}(\mathrm{p}, \mathrm{xn}){ }^{250-\mathrm{x}}$ Es reactions in comparison with previously reported values . . . . . . . . . . . . . . . 147

7.1 Average or most probable TKE vs $Z^{2} / A^{1 / 3}$ for delayed and spontaneous

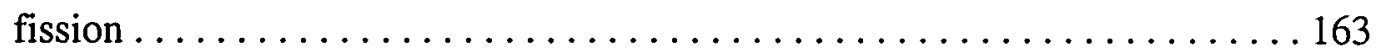

7.2 Probability of delayed fission vs $\mathrm{Q}_{\mathrm{EC}}$ .167 


\section{List of Tables}

3.1 Nuclides with reported ECDF branches and their $\mathrm{Q}_{\mathrm{EC}}$ and $\mathrm{P}_{\mathrm{DF}}$ values .....23

3.2 Decay properties of neutron-deficient einsteinium and californium isotopes . . 26

$4.1 \quad{ }^{249} \mathrm{Cf}$ target thickness . . . . . . . . . . . . . . . . . . . . . 40

4.2 Reactions and beam energies for the production of einsteinium isotopes . . . . 42

6.1 Properties of the kinetic energy and mass distributions for the ECDF of ${ }^{242}$ Es . .67

6.2 Properties of the kinetic energy and mass distributions for the ECDF of ${ }^{244}$ Es . .89

6.3 Fission rates versus projectile energy for the ${ }^{233} \mathrm{U}\left({ }^{14} \mathrm{~N}, \mathrm{xn}\right){ }^{247-\mathrm{x}} \mathrm{Es}$ reactions $\ldots 107$

6.4 $\mathrm{CN}$ recoil energies, effective target thickness and energy loss for protons . . . 125

7.1 Summary of single particle level assignments and $\log f t$ values . . . . . . . 168

7.2 Experimentally determined cross sections for the production of einsteinium . 171 


\section{Acknowledgments}

First and foremost I would like to thank my research advisor, Dr. Darleane Hoffman, for all of her help and encouragement during my tenure in her group. Thanks for letting me wander into your office when I had a question or needed advice. I promise to try and eradicate the word "nuculer" from everyone's vocabulary.

Next I would like to thank Dr. Kenneth Gregorich for all of his valuable help and assistance. I certainly would not have been able to accomplish all that I did without his guidance. Thanks for being there. I also need to thank Diana Lee. She was responsible for my understanding of so many different things. Thanks for all of your help and patience.

I also need to thank the operators past and present of the 88-Inch Cyclotron at Lawrence Berkeley National Laboratory. None of my experiments would have ever happened without their expertise. They must also be praised for their patience during an experiment, when I would wander into the control room every 10 minutes asking, "Is there beam yet?"

Over the years I have had the pleasure and privilege of working with some truly wonderful and gifted graduate students and postdocs. I thank all of them for their friendship, guidance, advice and assistance during experiments. I would also like to specifically thank a few of them, in no particular order. Thanks to Bobby Kadkhodayan for helping me decide to stay here at Berkeley and join the Hoffman group. I couldn't 
imagine myself anywhere else. Also thanks for showing me how to make targets. Thanks to Eric Sylwester for always being a good friend and a good poker buddy. I am especially indebted to Eric for introducing me to my husband, and I hope his friendship will always be a part of our lives. Thanks to Michael Lane for serving as comic relief when I really needed it and for driving us all to lunch on occasion. He is a good friend and I hope he always will be. (Please don't spend your rent money on electronics equipment!) And finally, I would like to thank the current trio of veteran students in the group, Joshua Patin, Dan Strellis and Philip Wilk. I'm glad they were around during these last few months when the stress levels were getting out of hand. Our lunchtime conversations (usually centered on sports of some kind) and poker games really gave me some much needed diversions. I wish them all the best of luck with the remainders of their graduate careers and with their future endeavors.

Finally, I would like to especially thank my family for all of their love and encouragement. Thanks to Mom for always listening when I needed somebody's ear, and thanks for never asking, "Are you done yet?" Your love and support were very important to me and were necessary to help keep me going. And I especially want to thank my husband David for everything that he has done over these past years. I certainly could not have done it without you and your love and encouragement. I love you with all of my heart and soul. This dissertation is dedicated to you for your unwavering love and patience. Thank you also for being so proud of me. 


\section{Introduction}

\subsection{Discovery of Einsteinium}

The discovery of einsteinium (element 99) was an unexpected result from the detonation of the first thermonuclear device, "Mike", which took place on November 1, 1952 [GHI55]. "Mike" was detonated on the Eniwetok Atoll in the Pacific Ocean, and destroyed most of the atoll in the process. Following the detonation, debris from the explosion was collected on filter papers, which were attached to airplanes that flew through the resulting debris clouds. Later, fall-out material was collected from the surface of a neighboring atoll. These samples were brought to several U. S. laboratories for detailed chemical analyses.

Some of the debris and fall-out samples were run through cation-exchange columns using ammonium citrate as the eluting agent. Ammonium citrate elutes trivalent ( $3+$ oxidation state) actinides in reverse order of their atomic numbers, so that the heaviest actinides are eluted from the column first, and americium (element 95) is eluted last. Two new $\alpha$-activities were detected in the elutions preceding the fraction that contained element 98 (californium), indicating the presence of two new elements with mass numbers greater than 98 . The first unknown activity was fermium (element 100.) The second activity showed a 20-day half-life and had an $\alpha$-particle energy of $6.6 \mathrm{MeV}$. This was identified as ${ }^{253}$ Es.

Scientists theorized that elements 99 and 100 were produced when the ${ }^{238} U$ in the "Mike" device captured a very large number of neutrons. The resulting heavy nuclides then underwent successive beta-decays, creating elements with larger atomic numbers. It 
was determined that the uranium was exposed to approximately $1-4 \times 10^{24}$ neutrons [GHI55] over the course of only a few nanoseconds! To form ${ }^{253} \mathrm{Es}$, the ${ }^{238} \mathrm{U}$ first had to capture 15 neutrons, forming ${ }^{253} U$. This was then followed by a series of seven betadecays, ultimately forming the beta-stable nuclide ${ }^{253} \mathrm{Es}$. The ${ }^{255} \mathrm{Fm}$ also discovered in the debris from the explosion was formed in the same way, except that the ${ }^{238} \mathrm{U}$ first captured 17 neutrons and then underwent eight beta-decays, ultimately forming ${ }^{255} \mathrm{Fm}$. This type of neutron capture process is described in more detail in section 1.2.

It wasn't until 1961 that a macroscopic and weighable amount of einsteinium was isolated [SEA90]. Workers at Berkeley separated a small fraction of pure ${ }^{253}$ Es following an intense neutron bombardment of ${ }^{239} \mathrm{Pu}$. Ultimately, the amount of einsteinium that was isolated after the bombardment was only a few hundredths of a microgram.

\subsection{Electron-Capture Delayed Fission}

Delayed fission (DF) is a decay mode whereby a parent nucleus populates excited states in the daughter nucleus, which then fission. Beta-delayed fission (BDF) and electron-capture delayed fission (ECDF) occur when the parent first undergoes betadecay or electron-capture (EC) decay, respectively. ECDF is the decay mode discussed in this dissertation. A schematic of the ECDF process is shown in Figure 1.1. The excited states in the daughter nucleus populated by the initial EC-decay can be above the fission barrier, resulting in prompt fission, within the second well of the potential energy surface, yielding a fission shape isomer, or within the first well of the potential energy surface, resulting in an electromagnetic isomer. 


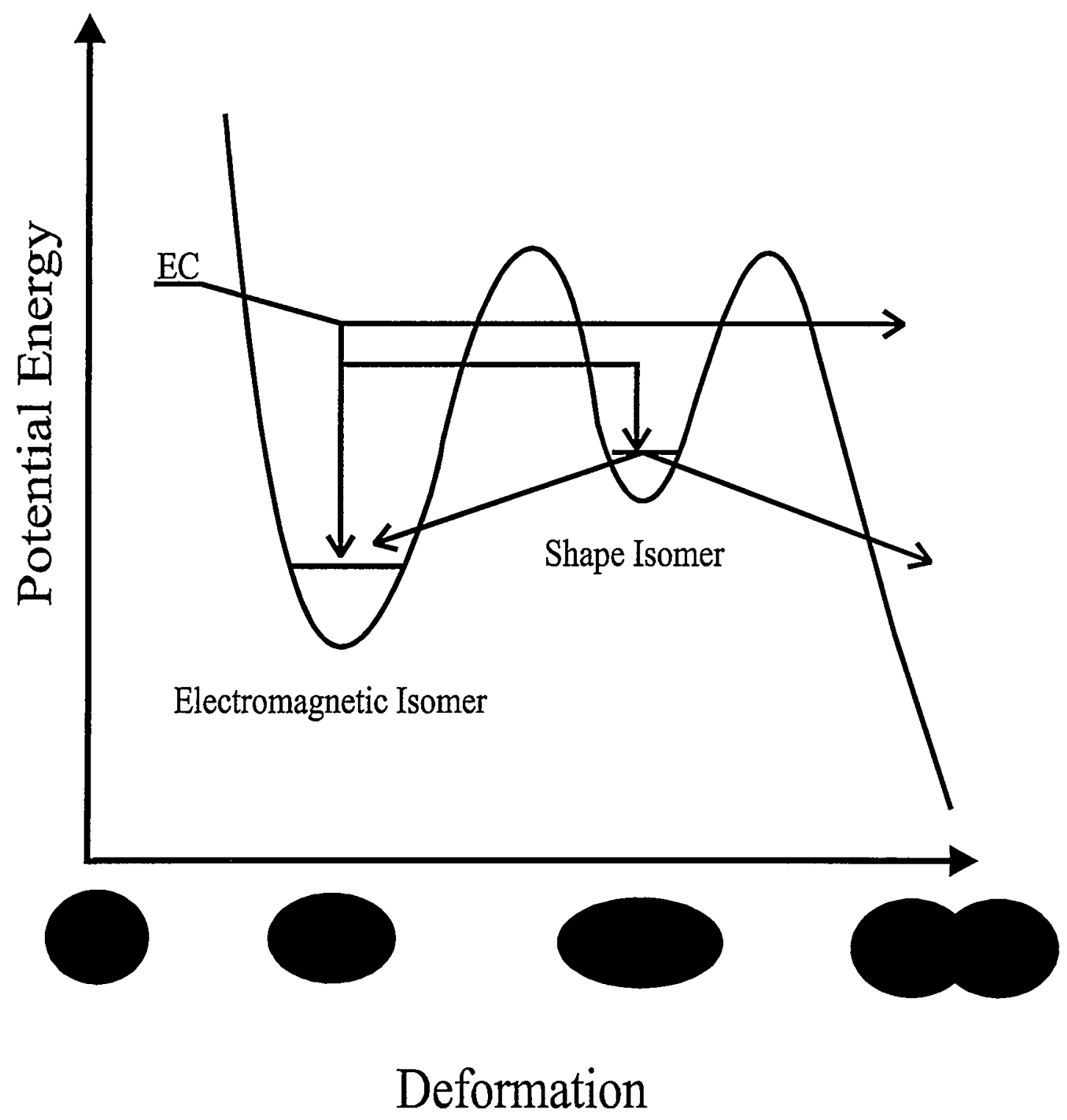

Figure 1.1: Two-dimensional drawing of the delayed fission process. The potential energy surface of the daughter nucleus is shown, illustrating the double-humped fission barrier commonly observed in the actinides. As seen in the figure, the fission can occur from either a high-lying state in the first potential well, or from an isomeric state in the second well. 
ECDF becomes an important decay mode when the energy released during the EC-decay (the electron-capture $\mathrm{Q}$-value, or $\mathrm{Q}_{\mathrm{EC}}$ ) is comparable to the height of the fission barrier [HAL89]. The EC-decay will predominantly populate states within the first well, because the population of states in the second well would require a significant change in nuclear deformation occurring simultaneously with the EC-decay. This can be seen in Figure 1.1. Once high-lying states in the daughter are occupied, tunneling through the first barrier to the second well must compete with $\gamma$-ray transitions leading to lower states in the first well. Once the nucleus has tunneled into the second well (forming a shape isomer), tunneling through the remainder of the fission barrier and undergoing fission must compete with $\gamma$-decay inside the second well and the possibility of tunneling back through the barrier to the first well. A shape isomer exists because of the second minimum in the potential energy surface seen in Figure 1.1. The shape isomer is characterized by a very large prolate deformation with a major to minor semiaxis ratio of approximately 2:1 [POE89].

ECDF was first seen (unknown to the researchers conducting the experiments) in 1966 in the light americium and neptunium regions [KUZ66, KUZ67]. Fission tracks with half-lives on the order of minutes were detected in these experiments, but they were thought to originate from spontaneous fission branches in americium and neptunium. These fission events were later attributed to ECDF in 1972 by Skobelev et al. [SKO72], after Berlovich and Novikov [BER69] had reported in 1969 that the nuclei in question met the requirements for a new delayed fission mode. In 1978, Habs et al. [HAB78] confirmed the existence of a type of fission decay in ${ }^{232} \mathrm{Am}$, and in 1989, Hall et al. [HAL89B] provided the first direct evidence for the existence of ECDF in ${ }^{232} \mathrm{Am}$ by 
measuring fission fragments in coincidence with $\mathrm{x}$-rays that originated from the initial EC-decay.

There are three primary reasons why ECDF and delayed fission processes in general are studied. First of all, beta-delayed fission is thought to have an important role in determining the yields of heavy elements produced in multiple neutron capture processes such as the astrophysical r-process and in nuclear weapons tests [BUR57, WEN74, WEN75, KLA81, MEY89]. These types of processes were briefly mentioned in section 1.1 in conjunction with the discovery of einsteinium. R-process nucleosynthesis, which occurs during supernova explosions, is responsible for the creation of the heaviest elements that are found in nature, such as uranium. During the r-process or a nuclear weapon explosion, heavy target material is exposed to a large neutron flux for a brief period of time and captures a very large number of neutrons. This produces neutron-rich nuclides all the way out to the neutron drip-line. The nuclei then undergo multiple betadecays until either a beta-stable nuclide is achieved or a fission event terminates the chain. With every successive beta-decay, the nucleus increases its atomic number, forming higher $\mathrm{Z}$ elements. If this chain of beta-decays were to continue, we would observe heavy actinides, and possibly even superheavy elements in amounts larger than those observed in nature or in nuclear weapons tests [HOFF86, HOFF88]. It is believed that BDF terminates the chain of beta-decays before heavy elements can be achieved. Hoff [HOFF86, HOFF88] has shown that previous calculations had actually overestimated the amount of BDF occurring in decay chains beginning with neutron-rich uranium, especially in nuclides with mass number 252 to 257 . However, in 1992, Staudt and Klapdor-Kleingrothaus [STA92] developed a new model, which confirmed that BDF 
probabilities had indeed been overestimated in the region $A=248-257$, but also confirmed that the influence of $B D F$ increases at $A=257$. Since certain actinide pairs are used as cosmochronometers in determining the age of the galaxy [THE83, YOK83, MEY86], the influence that DF has on the production of these actinides is of scientific interest.

The delayed fission process can also be used as a probe of the structure of the fission barrier. The probability of delayed fission is related to the Q-value of the parent beta- or EC-decay, and to the height and curvature of the fission barrier [HAB78]. If the delayed fission probability can be measured experimentally, then information about the fission barrier can be extracted from this value. During delayed fission it is also possible that excited states in the fission shape isomer can be populated (the second potential energy well in Figure 1.1.) These excited states are populated with the half-life of the parent, which can be on the order of minutes, making it possible to study the highly deformed shape isomer in an out-of-beam environment where the background is significantly reduced [HAL89]. Studying the decay of the shape isomer will also give new insight into the structure of the fission barrier.

The final reason why delayed fission is studied is also the purpose of the experiments described in this dissertation. Delayed fission allows study of fission properties in regions of the chart of the nuclides where spontaneous fission branches are negligible. In the case of ECDF, the even-proton, even-neutron daughter nuclei created after the initial EC-decay of odd-odd nuclides are very neutron deficient, with spontaneous fission half-lives ranging from days for ${ }^{242} \mathrm{Cf}$ to $10^{4}$ years for ${ }^{248} \mathrm{Cf}$. Figure 1.2 shows spontaneous fission half-lives for typical daughter nuclei formed via the ECDF process. By finding a parent precursor that undergoes ECDF, we are able to measure 


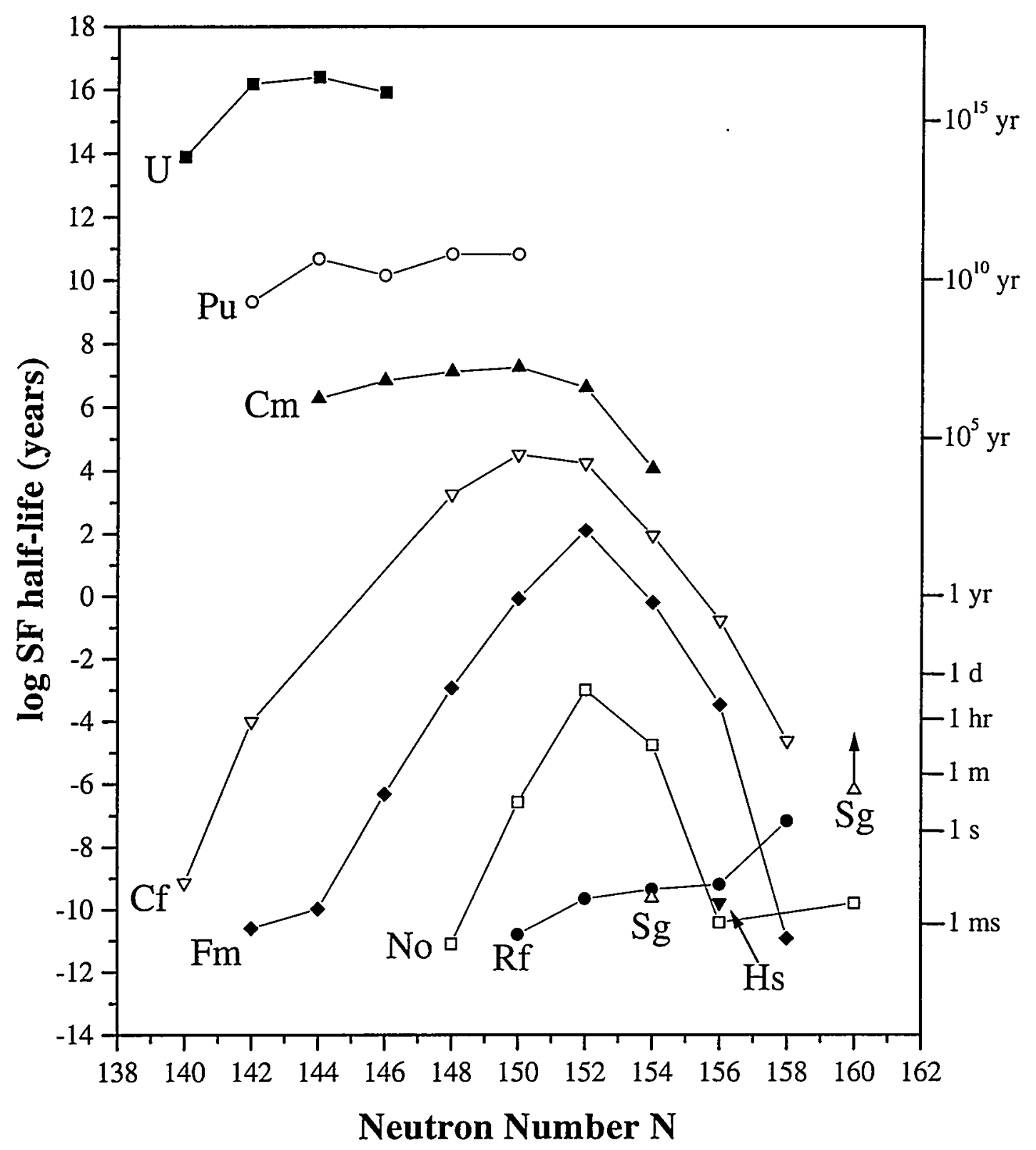

Figure 1.2: Spontaneous fission half-lives for even-proton, evenneutron isotopes versus neutron number. 
kinetic energy and mass-yield distributions of very neutron deficient actinides such as ${ }^{242} \mathrm{Cf}-{ }^{248} \mathrm{Cf}$, which we would otherwise be unable to do.

\subsection{Isotope Production}

Since einsteinium is not a naturally occurring element, it must be synthesized in order to study its properties. If one has access to a nuclear reactor, neutron-rich isotopes of einsteinium can be produced via neutron capture by exposing uranium or heavier actinides to a large neutron flux. However, this method works best for producing longerlived isotopes of einsteinium, since the material has to be removed from the reactor following neutron bombardment, and a chemical separation must be done to isolate the einsteinium from other nuclides produced via neutron capture. Neutron-deficient einsteinium isotopes, which are also shorter-lived, must be produced via a compound nucleus reaction.

A common method for the production of einsteinium, actinides and transactinide elements is to bombard an actinide target with a projectile beam. The incident nuclei must be energetic enough to overcome the Coulomb barrier $\left(V_{c}\right)$ between the target and projectile nuclei given by the following equation:

$$
V_{c}=\frac{Z_{a} Z_{A} e^{2}}{R_{a}+R_{A}}
$$

where $Z_{a}$ is the atomic number of the projectile, $Z_{A}$ is the atomic number of the target, $e$ is the electronic charge, $R_{a}$ is the nuclear radius of the projectile and $R_{A}$ is the nuclear radius of the target. The incident beam nucleus approaches the target at a certain distance from a line that goes through the target nucleus and is parallel to the beam direction. The 
distance normal to this line is called the impact parameter [KRA88]. At small impact parameters, the projectile nucleus fuses with the target nucleus to form a compound nucleus (CN), which represents the complete fusion of the two nuclei [FRI81]. The $\mathrm{CN}$ exists for $10^{-14}-10^{-19} \mathrm{~s}$ [FRI81], making it a quasi-stationary intermediate state. The $\mathrm{CN}$ is formed with considerable excitation energy (approximately $40-50 \mathrm{MeV}$ for $\mathrm{A}_{\mathrm{CN}}<300$ [SEA90]) and it predominantly deexcites via particle emission or fission. According to the compound nucleus theory, once it is formed the $\mathrm{CN}$ completely forgets its method of production, and the probability of decay into a specific set of final products depends only on the total energy of the system, not on the mode of formation. The $\mathrm{CN}$ reaction is shown below:

$$
\mathrm{a}+\mathrm{X} \rightarrow \mathrm{C}^{*} \rightarrow \mathrm{Y}+\mathrm{b}
$$

where a and $\mathrm{X}$ represent the projectile and target, respectively, $\mathrm{C}^{*}$ is the compound nucleus plus excitation energy, $\mathrm{Y}$ is the final target-like product and $\mathrm{b}$ represents the particles emitted during the decay of the $\mathrm{CN}$. Since deexcitation of the $\mathrm{CN}$ via fission is also possible, $\mathrm{Y}$ and $\mathrm{b}$ can also represent fission fragments. Each emitted particle carries away a fraction of the available excitation energy so that several particles may be evaporated from the $\mathrm{CN}$ before complete deexcitation occurs. Deexcitation via neutron emission is more probable than charged particle emission because neutrons do not have to overcome the Coulomb barrier given by equation 1.1 . Even in cases where the $\mathrm{CN}$ is neutron-deficient, neutron emission is still the preferred decay mode [KRA88].

The relative probability for a nuclear reaction to occur is given by the cross section $(\sigma)$ [FRI81], which has dimensions of area. Using a classical description, the probability of a reaction occurring between a target nucleus and an incident particle is 
proportional to the cross-sectional area presented by the target nucleus. The cross section of a compound nucleus reaction forming a particular isotope is given by

$$
\sigma=\frac{\mathrm{R}}{\mathrm{IN}}
$$

where $\mathrm{R}$ is the production rate of the isotope of interest, $\mathrm{I}$ is the particle flux (the number of incident particles per unit time), and $\mathrm{N}$ is the number of target nuclei per unit area. Cross sections are given in units of barns (b) where one barn is equal to $1 \times 10^{-24} \mathrm{~cm}^{2}$. Typical CN reactions used to produce actinides have cross sections that range from millibarns (mb) down to picobarns (pb).

It is important to have an estimate of the magnitude of the cross section for the nuclear reaction of interest. If the cross section is too small, only a few atoms may be produced over the course of several weeks, making it nearly impossible to detect the nuclide of interest. If a particular cross section is unknown, there are ways to determine whether or not the nuclear reaction is feasible. An initial estimate of the cross section can be obtained by using one of several different computer codes that model each step of the compound nucleus reaction. These codes typically give estimates of cross sections for the production of isotopes at several different incident beam energies. The magnitudes of cross sections can also be measured from an experimental determination of the excitation function. An excitation function is the relationship between a particular reaction cross section and incident beam energy [FRI81]. During an excitation function experiment, the beam energy is varied over a particular range and the production cross section of the nuclide of interest is measured at each energy. This allows for the determination of the optimal energy to use in order to maximize production of a certain isotope. Also, it is important to know which energy to use during an experiment in order to optimize the use 
of cyclotron time. Excitation functions are extremely valuable in the production of actinide and transactinide elements. 


\section{Theory of Delayed Fission}

\subsection{Calculations of the Delayed Fission Probability}

The chance that a particular nucleus will undergo delayed fission is given by the probability of delayed fission $\left(\mathrm{P}_{\mathrm{DF}}\right)$. We are assuming that beta- or EC-decay will result in a delayed fission event, and $\alpha$ - or $\gamma$-decay will not. This quantity can be determined experimentally by comparing the number of beta- or EC-decays that result in fission, $\mathrm{N}_{\mathrm{if}}$, to the total number of beta- or EC-decays, $\mathrm{N}_{\mathrm{i}}$. This is illustrated in the following equation,

$$
P_{D F}=\frac{N_{i f}}{N_{i}}
$$

where i can be either beta or EC.

The $\mathrm{P}_{\mathrm{DF}}$ can also be estimated from theoretical considerations. It depends on the Q-value for the decay of interest $\left(Q_{i}\right.$ where $i$ is either beta or $\left.E C\right)$, a transition probability function for the decay of interest $\left(W_{i}(E)\right.$ where $E$ is the excitation energy of the daughter nucleus), and the ratio of the fission decay width to the total decay width of excited levels in the daughter nucleus $\left(\Gamma_{\mathrm{f}} / \Gamma_{\mathrm{l}}(\mathrm{E})\right)$. It is assumed that only $\gamma$-decay and fission deexcitation compete in the daughter nucleus, since the decay width for particle emission is much smaller in comparison [HAL89] (this assumption requires that excited states in the daughter are below the particle binding energy.) Therefore, the ratio of decay widths can be approximated as follows:

$$
\frac{\Gamma_{\mathrm{f}}}{\Gamma_{\mathrm{t}}}(\mathrm{E}) \approx \frac{\Gamma_{\mathrm{f}}}{\Gamma_{\mathrm{f}}+\Gamma_{\gamma}}(\mathrm{E})
$$


where $E$ is the excitation energy of the daughter nucleus and particle emission has been neglected. To determine the $\mathrm{P}_{\mathrm{DF}}$, all of these factors must be integrated over the total energy available for the decay $\left(\mathrm{Q}_{\mathrm{i}}\right)$ and normalized to the total number of beta- or ECdecays. Equation 2.3 shows the theoretical expression for the delayed fission probability.

$$
P_{D F}=\frac{\int_{0}^{Q_{1}} W_{i}\left(Q_{i}-E\right) \frac{\Gamma_{f}}{\Gamma_{f}+\Gamma_{\gamma}}(E) d E}{\int_{0}^{Q_{i}} W_{i}\left(Q_{i}-E\right) d E}
$$

Equation 2.3 works in the limit that the level density in the daughter is high enough to assume a continuum of states. Otherwise, the integrals should be replaced with summations if discrete states in the daughter are considered.

From this point on, only EC will be considered because ECDF is the delayed fission process discussed throughout this dissertation. In the region where ECDF occurs, $\mathrm{Q}_{\mathrm{EC}}$ values are large enough that there are high level densities in the EC-daughters, and the EC-decay can be discussed in statistical terms [DUK70]. Therefore, the transition probability function in equation $2.3, \mathrm{~W}_{\mathrm{EC}}(\mathrm{E})$, can be expressed as the product of the integrated Fermi function, $f$, which determines the rate of the EC-decay, and the beta strength function, $S_{\beta}$, which determines the average distribution of states populated by the EC-decay at an excitation energy E [KLA79]. This is given below in equation 2.4.

$$
\mathrm{W}_{\mathrm{EC}}(\mathrm{E}) \approx f\left(\mathrm{Q}_{\mathrm{EC}}-\mathrm{E}, \mathrm{Z}\right) \mathrm{S}_{\beta}(\mathrm{E})
$$

For EC transitions the Fermi function can be approximated as $\left(Q_{E C}-E\right)^{2}$ [DUK70]. The quantity $\left(\mathrm{Q}_{\mathrm{EC}}-\mathrm{E}\right)$ is the energy of the transition, neglecting the electron binding energy. $\mathrm{S}_{\beta}$ has been approximated using several different methods. It can be taken to be proportional to the nuclear level density in the EC-daughter [PAP72, SHA77], it can be 
determined from the gross theory of beta-decay [KOD75], or it can be considered to be a constant value above a given excitation energy and zero below [PAP72, KRA73, HOR75]. Klapdor et al. [KLA79] have pointed out that all of these assumptions ignore nuclear structure effects that modify the simple statistical strength function. Experimental evidence for these effects has been derived from beta-delayed neutron emission experiments [KRA76, KRA79]. Klapdor used a microscopic model to calculate $S_{\beta}$ and found that these structure effects had a significant impact on the value of $P_{D F}$ obtained. Even so, for a qualitative understanding of $\mathrm{P}_{\mathrm{DF}}$, treating $\mathrm{S}_{\beta}$ as a constant above a certain cut-off energy is acceptable.

In order to understand just how the form of $S_{\beta}$ can affect the delayed fission probability, consider the following two examples. First of all, if $S_{\beta}$ is considered a constant above the cut-off energy, a large $\mathrm{Q}_{E C}$ relative to the height of the fission barrier would imply a large $\mathrm{P}_{\mathrm{DF}}$. However, if EC-decay to the ground state in the daughter is superallowed (no change in spin or parity) with a low $\log f t$ value, then high-lying states in the daughter would be populated with low probability and the Fermi function in euqation 2.4 would be very low. Therefore, even though $\mathrm{S}_{\beta}$ may be large, the $\mathrm{P}_{\mathrm{DF}}$ would still be small, whereas one would initially assume a large $P_{D F}$ based solely on the value of $\mathrm{S}_{\beta}$. In another example, Hall et al. [HAL89C] observed beta-delayed fission in ${ }^{256} \mathrm{Es}^{m}$ from a single level $1425 \mathrm{keV}$ above the ground state. The assumption of a constant $\mathrm{S}_{\beta}$ would predict no delayed fission branch in this nuclide, but in this case $\gamma$-decay from the $1425-\mathrm{keV}$ level was hindered such that fission was able to compete. In both of these examples, ignoring the structure inherent to the beta-strength function would result in incorrectly predicting the delayed fission branch. 
More recently, Meyer et al. [MEY89] calculated $\mathrm{S}_{\beta}$ microscopically with a code that explicitly treated nuclear deformation. By including nuclear deformation in his model, he typically calculated lower beta-delayed fission branches than previously reported for certain nuclides. Nuclear deformation tends to spread out the beta-strength among daughter states leading to more decay to lower-lying states and therefore less beta-delayed fission [MEY89]. To really determine the $\mathrm{P}_{\mathrm{DF}}$ using theoretical considerations, a beta-strength function must be used that not only considers nuclear structure effects, but also considers nuclear deformation. Otherwise, the probability determined using these equations does not match experimental results.

Further approximations can be made to equation 2.3. The $\gamma$-decay width, $\Gamma_{\gamma}(E)$, can be estimated [GAN80] from the probability for $\gamma$-ray transitions, $P_{\gamma}$, as in equation 2.5 ,

$$
\Gamma_{\gamma}(\mathrm{E})=\frac{\mathrm{P}_{\gamma}}{2 \pi \rho(\mathrm{E})}=\frac{\mathrm{C}_{\gamma} \Theta^{4} \mathrm{e}^{(\mathrm{E} / \Theta)}}{2 \pi \rho(\mathrm{E})}
$$

where $\rho(E)$ is the nuclear level density, $C_{\gamma}$ is a constant with the value $9.7 \times 10^{-7} \mathrm{MeV}^{-4}$ and $\Theta$ is the nuclear temperature $(0.5-0.6 \mathrm{MeV})$. The nuclear temperature can be calculated from formulas like the ones in [SWI83].

The fission width term, $\Gamma_{\mathrm{f}}(E)$, can be derived from the penetrability of the fission barrier in a similar manner. This yields equation 2.6 ,

$$
\Gamma_{f}(E)=\frac{P_{f}}{2 \pi \rho(E)}
$$

where $P_{f}$ is the penetrability of the entire double-humped fission barrier (see Figure 1.1.) The calculation of the penetrability of the entire fission barrier in the actinides can be 
difficult because the fission barriers tend to be complex. Therefore, it is common to simplify the penetrability [HAB78, GAN80] through the double-humped barrier by approximating $\mathrm{P}_{\mathrm{f}}$ to be

$$
\mathrm{P}_{\mathrm{f}} \approx \mathrm{P}_{\mathrm{A}}(\mathrm{E}) \mathrm{R}_{\mathrm{B}}
$$

where $P_{A}(E)$ is the penetrability of the inner barrier and $R_{B}$ is the transmission coefficient for fission from the lowest state in the second well. This assumption requires that all nuclear motion in the second well be strongly damped, meaning that all states populated in the second well have time to undergo $\gamma$-decay to the ground state of the second well. before fission occurs. The penetrability of the outer barrier from a single level is reduced to a calculation involving the simple transmission coefficient $R_{B}$. The penetrability of the inner barrier can be calculated using the Hill-Wheeler formalism of a parabolic barrier [HIL53]. Substituting the Hill-Wheeler formula into equation 2.7 results in the version of $\Gamma_{\mathrm{f}}$ given in equation 2.8 .

$$
\Gamma_{f}(E) \approx \frac{R_{B}}{2 \pi \rho(E)}\left(1+e^{\frac{2 \pi\left(B_{t}-E\right)}{\hbar \omega_{t}}}\right)^{-1}
$$

In equation $2.8, \mathrm{~B}_{\mathrm{f}}$ is the inner barrier height, $\hbar \omega_{\mathrm{f}}$ is the energy associated with the curvature of the inner barrier and $E$ is the excitation energy. The quantity $R_{B}$ is comprised of the ratio of transmission coefficients for the lowest level in the second well for tunneling through the outer barrier to undergo fission to that for undergoing $\gamma$-decay back to the first potential well. Using equations 2.5 and 2.8 , the ratio of the fission and $\gamma$ decay widths can be expressed as 


$$
\frac{\Gamma_{\mathrm{f}}}{\Gamma_{\gamma}+\Gamma_{\mathrm{f}}}(\mathrm{E}) \approx \frac{\mathrm{R}_{\mathrm{B}}\left(1+\mathrm{e}^{\frac{2 \pi\left(\mathrm{B}_{\mathrm{f}}-\mathrm{E}\right)}{\hbar \omega_{\mathrm{f}}}}\right)^{-1}}{\mathrm{C}_{\gamma} \Theta^{4} \mathrm{e}^{(\mathrm{E} / \Theta)}+\mathrm{R}_{\mathrm{B}}\left(1+\mathrm{e}^{\frac{2 \pi\left(\mathrm{B}_{\mathrm{f}}-\mathrm{E}\right)}{\hbar \omega_{\mathrm{r}}}}\right)^{-1}}
$$

which illustrates the dependence of this term on the energy available for decay and the structure of the fission barrier (see equation 2.3). This also illustrates that if the delayed fission probability is known, information about the fission barrier can be derived from that quantity.

Using all of the approximations presented so far, equation 2.3 can be rewritten in its simplest form, equation 2.10:

$$
P_{D F} \approx \frac{\int_{C}^{Q_{E C}}\left(Q_{E C}-E\right)^{2} \frac{\Gamma_{f}}{\Gamma_{\gamma}+\Gamma_{f}}(E) d E}{\int_{C}^{Q_{E C}}\left(Q_{E C}-E\right)^{2} d E}
$$

where $C$ is the cut-off energy below which $S_{\beta}$ is assumed to be zero. Since all nuclides that undergo ECDF are odd-odd, $\mathrm{C}$ is equal to $26 / \sqrt{\mathrm{A}} \mathrm{MeV}$ [KRA73] where $\mathrm{A}$ is the mass number of the fissioning nucleus. The denominator can be easily evaluated, resulting in the final form of $\mathrm{P}_{\mathrm{DF}}$, equation 2.11.

$$
\mathrm{P}_{\mathrm{DF}} \approx \frac{\int_{C}^{\mathrm{Q}_{\mathrm{CC}}}\left(\mathrm{Q}_{\mathrm{EC}}-\mathrm{E}\right)^{2} \frac{\Gamma_{\mathrm{f}}}{\Gamma_{\gamma}+\Gamma_{\mathrm{f}}}(\mathrm{E}) \mathrm{dE}}{\frac{1}{3}\left(\mathrm{Q}_{\mathrm{EC}}-26 \mathrm{~A}^{-1 / 2}\right)^{3}}
$$

The delayed fission probability has a rough exponential dependence on the difference between the height of the fission barrier and the energy available for decay, the $\mathrm{Q}_{\mathrm{EC}}$. This dependence comes from the $\Gamma_{\mathrm{f}}$ term and directly influences the $P_{D F}$. It should be 
noted that the derivations presented throughout this chapter represent only the most basic theoretical expressions pertaining to the delayed fission probability. For a more complex theoretical derivation, the reader is referred to the references quoted in the text.

\subsection{Requirements for ECDF}

Because of the dependence the $\mathrm{P}_{\mathrm{DF}}$ has on the difference between the fission barrier height and the $\mathrm{Q}_{\mathrm{EC}}$ as discussed in section 2.1, for delayed fission to become a prominent decay mode the $\mathrm{Q}_{\mathrm{EC}}$ must be at least comparable to $\mathrm{B}_{\mathrm{f}}$. Fission barriers in the actinides typically range from $4-6 \mathrm{MeV}$ [HAL89], so isotopes with $\mathrm{Q}_{\mathrm{EC}}$ values greater than 4 $\mathrm{MeV}$ should have measurable ECDF branches [HAL89]. Nuclides that meet this requirement are found in the neutron deficient actinides with odd numbers of protons and neutrons. These odd-odd nuclei have enhanced $\mathrm{Q}_{\mathrm{EC}}$ values associated with their $\mathrm{EC}$ decay to more stable even-even daughter nuclei. Figure 2.1 shows the region of the actinides where ECDF is expected to become a significant decay mode.

By looking at equation 2.11 , one would expect that the delayed fission probability would increase with increasing $\mathrm{Q}_{E C}$ [HAL89]. With more energy available for EC-decay, higher-lying states in the daughter nucleus would be populated, leading to an enhanced probability of undergoing fission. This has been confirmed experimentally. Figure 2.2 shows that the experimentally measured $\mathrm{P}_{\mathrm{DF}}$ values increase with $\mathrm{Q}_{\mathrm{EC}}$. Since the delayed fission probability can never be greater than one, current experiments are studying ECDF in nuclides with larger $\mathrm{Q}_{E C}$ values to determine at which energy the $\mathrm{P}_{\mathrm{DF}}$ function in Figure 2.2 levels off. Ultimately future experiments will be required to determine the shape of the relationship between $\mathrm{P}_{\mathrm{DF}}$ and $\mathrm{Q}_{\mathrm{EC}}$ seen in Figure 2.2. 


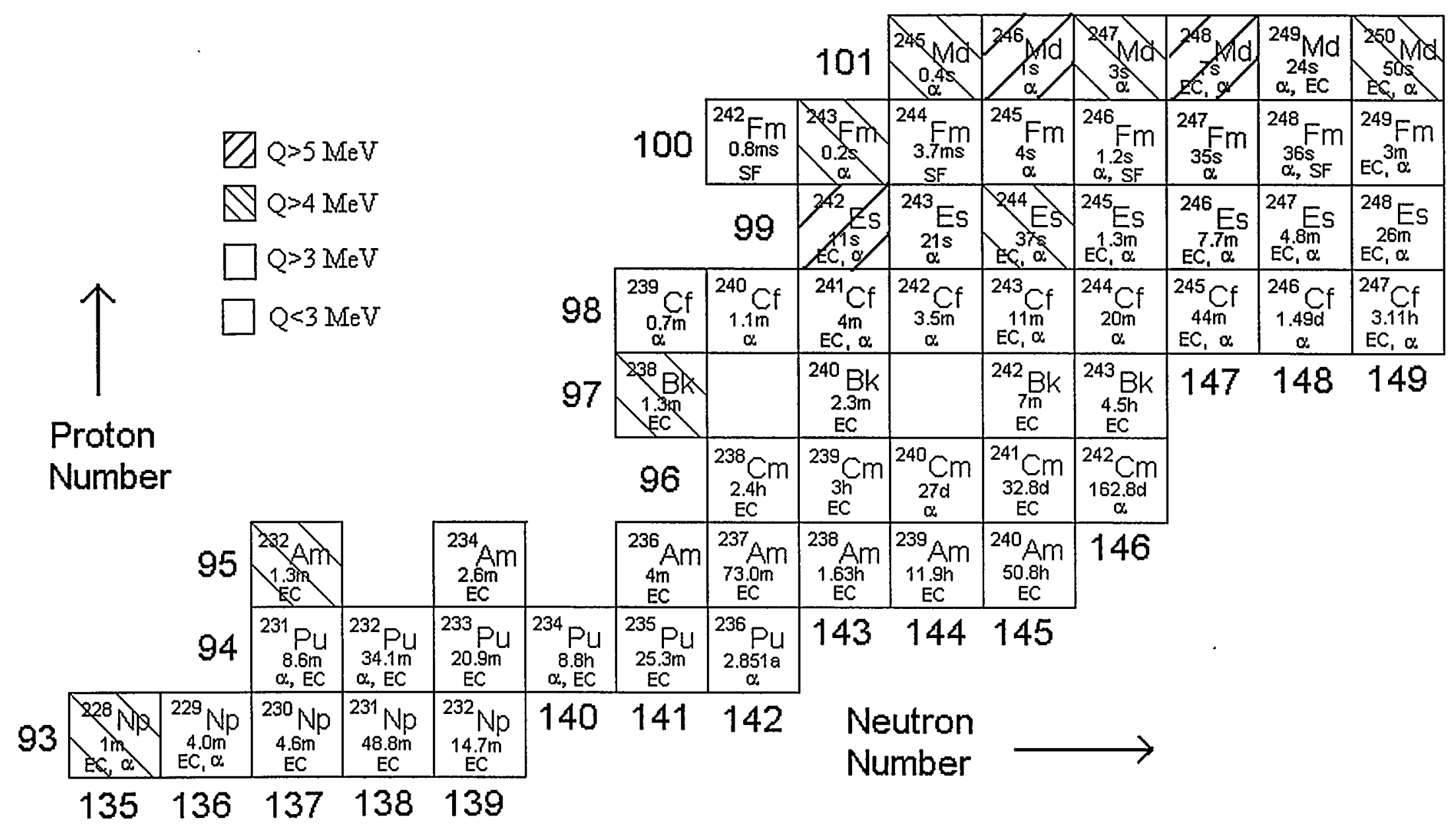

Figure 2.1: Portion of the table of isotopes showing actinide nuclei most likely to undergo ECDF. 


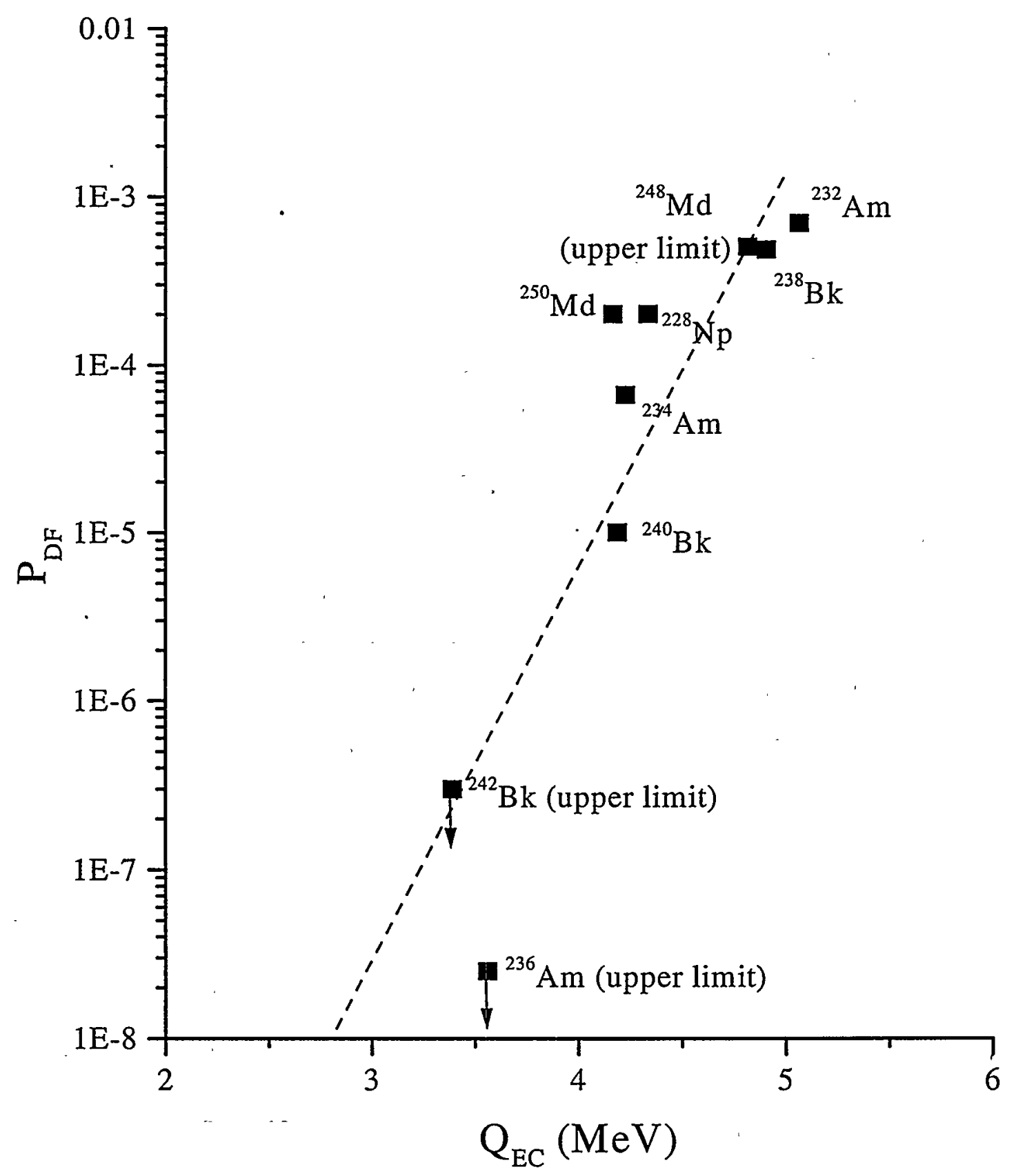

Figure 2.2: Experimentally measured $\mathrm{P}_{\mathrm{DF}}$ values versus $\mathrm{Q}_{\mathrm{EC}}$. $\mathrm{Q}$-values were calculated from the masses in [MÖL95]. Data are from References [GAN80, HAL89, HAL90, HAL90A, KRE94, KRE94A]. The line is to guide the eye and is not meant to be a fit to the data. 


\section{Previous Work}

\subsection{Previous Delayed Fission Studies}

ECDF in the einsteinium region was first reported by Gangrskii et al. in 1980 [GAN80]. The $P_{D F}$ values of ${ }^{244} E s,{ }^{246} E s$ and ${ }^{248}$ Es were reported, along with their corresponding production cross sections. The heights of the inner fission barriers of their respective EC-daughters were also estimated using formulas that were presented in the text [GAN80]. ${ }^{244}$ Es was produced both via the ${ }^{235} \mathrm{U}\left({ }^{14} \mathrm{~N}, 5 \mathrm{n}\right){ }^{244} \mathrm{Es}$ and ${ }^{237} \mathrm{~Np}\left({ }^{12} \mathrm{C}\right.$, $5 n)^{244}$ Es reactions at projectile energies of $82-86 \mathrm{MeV}$, although it was not specified which reaction was ultimately used to determine the $\mathrm{P}_{\mathrm{DF}} \cdot{ }^{246} \mathrm{Es}$ and ${ }^{248} \mathrm{Es}$ were both produced by bombarding ${ }^{238} \mathrm{U}$ with ${ }^{14} \mathrm{~N}$ (92-94 MeV and $74-76 \mathrm{MeV}$, respectively) followed by evaporation of either six or four neutrons. The production cross sections of ${ }^{246} \mathrm{Es}$ and ${ }^{248} \mathrm{Es}$ were reported to be $5 \mu \mathrm{b}$ and $6 \mu \mathrm{b}$, respectively, and the ${ }^{244} \mathrm{Es}$ cross section was said to be $1 \mu \mathrm{b}$, but again, it was never stated with which reaction this cross section was associated. The californium EC-daughters produced directly in the reactions via proton emission in the exit channels were neglected.

The $P_{D F}$ was determined [GAN80] for each isotope by comparing the number of observed fission events to the number of $\alpha$-decay events from the EC-daughter. While this is an acceptable method for measuring the probability of delayed fission, there were a few problems with their experimental methods that leave their $P_{D F}$ values in question. Fission fragments produced during the experiments were detected with the use of solidstate fission track detectors. After irradiation, these films were etched and visually scanned with a microscope. Four different sources of background events in these 
detectors were identified and thought to be eliminated using various techniques, but it is also possible that some of their fission events were indeed due to background. This method of detection does not allow for the determination of the fission properties of the EC-daughter. Also, no errors were given for their reported $\mathrm{P}_{\mathrm{DF}}$ values, making them estimates at best.

Three delayed fission events attributed to ${ }^{242}$ Es were reported in 1985 by Hingmann et al. [HIN85]. ${ }^{205} \mathrm{Tl}$ was bombarded with ${ }^{40} \mathrm{Ar}$ (beam energy not given) to produce ${ }^{242}$ Es with an estimated 40 -nb production cross section. Reaction products were separated from the beam and other background products using the SHIP velocity filter at the GSI laboratory in Darmstadt, Germany. $\alpha$-particles and fission fragments were detected using silicon detectors. An unknown $\alpha$-line at $7.85 \mathrm{MeV}$ was observed and assigned to ${ }^{242}$ Es. The half-life of this nuclide was estimated to be in the range of 5-25 s. Based on three observed fission events, the $\mathrm{P}_{\mathrm{DF}}$ was estimated. Hingmann et al. were also able to estimate a value for the height of the fission barrier of ${ }^{242} \mathrm{Cf}$ based on a previous analysis [HAB78]. Since their calculations were only based on three events, there are relatively large uncertainties associated with their results. Table 3.1 lists all previously reported cases of $E C D F$, along with their $\mathrm{P}_{\mathrm{DF}}$ values. 
Table 3.1. Nuclides that have reported ECDF branches, their $\mathrm{QEC}_{\mathrm{EC}}$ values and corresponding $\mathrm{P}_{\mathrm{DF}}$ values.

\begin{tabular}{|cccc|}
\hline Nuclide & $\mathrm{Q}_{\mathrm{EC}}{ }^{\mathrm{a}}(\mathrm{MeV})$ & $\mathrm{P}_{\mathrm{DF}}$ & Reference \\
\hline${ }^{228} \mathrm{~Np}$ & 4.34 & $2 \times 10^{-4} \pm 9 \times 10^{-5}$ & KRE94A \\
${ }^{232} \mathrm{Am}$ & 5.07 & $(6.9 \pm 1.0) \times 10^{-4}$ & HAL90A \\
${ }^{234} \mathrm{Am}$ & 4.23 & $(6.6 \pm 1.8) \times 10^{-5}$ & HAL90 \\
${ }^{236} \mathrm{Am}$ & 3.56 & $<2.5 \times 10^{-8} \mathrm{~b}$ & HAL89 \\
${ }^{238} \mathrm{Bk}$ & 4.91 & $(4.8 \pm 2.0) \times 10^{-4}$ & KRE94 \\
${ }^{240} \mathrm{Bk}$ & 4.19 & $10^{-5}$ & GAN80 \\
${ }^{242} \mathrm{Bk}$ & 3.39 & $<3 \times 10^{-7}$ & GAN80 \\
${ }^{242} \mathrm{Es}$ & 5.35 & $(1.4 \pm 0.8) \times 10^{-2}$ & HIN85 \\
${ }^{244} \mathrm{Es}$ & 4.36 & $10^{-4}$ & GAN80 \\
${ }^{246} \mathrm{Es}$ & 3.64 & $3 \times 10^{-5}$ & GAN80 \\
${ }^{248} \mathrm{Es}$ & 3.05 & $3 \times 10^{-7}$ & GAN80 \\
${ }^{248} \mathrm{Md}$ & 4.82 & $<5 \times 10^{-4}$ & GAN80 \\
${ }^{250} \mathrm{Md}$ & 4.17 & $2 \times 10^{-4}$ & GAN80 \\
\hline
\end{tabular}

${ }^{\mathrm{a}} \mathrm{Q}_{\mathrm{EC}}$ values are calculated from the most recent atomic masses given in Reference [MÖL95].

${ }^{\mathrm{b}}$ This value represents an upper limit only.

\subsection{Einsteinium Production and Excitation Functions}

The report of delayed fission in ${ }^{242}$ Es [HIN85] was also the first report of the production of this nuclide. In 1994, another group from GSI reported seeing ${ }^{242}$ Es as the $\alpha$-daughter of ${ }^{246} \mathrm{Md}$ [HOF94]. The ${ }^{209} \mathrm{Bi}\left({ }^{40} \mathrm{Ar}, 3 \mathrm{n}\right){ }^{246} \mathrm{Md}$ reaction was run at an energy of $197 \mathrm{MeV} . \alpha$-particles from ${ }^{246} \mathrm{Md}$ were observed, as well as the $\alpha$-decay of ${ }^{242}$ Es. A new $\alpha$-energy of $7.910 \mathrm{MeV}$ was assigned to the decay of ${ }^{242} \mathrm{Es}$, and its half-life was determined to be $40_{-20}^{+40} \mathrm{~s}$. These assignments were further refined in 1996 by Ninov et al. [NIN96] by using the same target and beam combination and the same energy. They assigned a half-life of $16_{-4}^{+6} \mathrm{~s}$ and an $\alpha$-energy of $7.920 \pm 0.020 \mathrm{MeV}$ to ${ }^{242}$ Es. The einsteinium was identified as the daughter of ${ }^{246} \mathrm{Md}$ using an $\alpha-\alpha$ correlation technique. As of this writing, these results were the last reported values for ${ }^{242}$ Es. 
${ }^{244}$ Es was first identified in 1970 by Eskola [ESK70] during an experiment in which a ${ }^{233} \mathrm{U}$ target was bombarded with ${ }^{15} \mathrm{~N}$ projectiles. This paper was a preliminary report, but ${ }^{244}$ Es was said to decay with a $100 \%$ EC-branch and a half-life of $40 \pm 5 \mathrm{~s}$. This dominant EC-branch was confirmed in 1973 when no $\alpha$-particles belonging to ${ }^{244}$ Es were observed during an experiment in which ${ }^{241} \mathrm{Am}$ was bombarded with ${ }^{12} \mathrm{C}$ to look for isotopes of mendelevium and their respective einsteinium daughters [ESK73]. A later paper by Eskola et al. [ESK73A] measured $\alpha$-particles from the decay of ${ }^{244}$ Es produced via the ${ }^{233} \mathrm{U}\left({ }^{15} \mathrm{~N}, 4 \mathrm{n}\right){ }^{244}$ Es reaction at projectile energies of $77-82 \mathrm{MeV}$. They assigned an $\alpha$-energy of $7.57 \pm 0.02 \mathrm{MeV}$ and a half-life of $37 \pm 4 \mathrm{~s}$ to ${ }^{244} \mathrm{Es}$, and reported an $\alpha$ branch of $4_{-2}^{+3} \%$, confirming that EC is still the dominant decay mode for this nuclide.

${ }^{246}$ Es and ${ }^{247}$ Es were first reported in 1954 [GHI54] but there was some uncertainty about their half-life and $\alpha$-energy assignments. In 1967 Mikheev et al. [MIK67] produced both of these nuclides using the same reaction that Ghiorso et al. used in $1954,{ }^{238} \mathrm{U}\left({ }^{14} \mathrm{~N}, \mathrm{xn}\right){ }^{252-\mathrm{x}}$ Es. In their paper, they presented excitation functions for the production of einsteinium nuclides. They were able to positively identify both isotopes, and were also able to determine their half-lives and $\alpha$-decay energies. ${ }^{246}$ Es was assigned a half-life of $7.7 \pm 0.5 \mathrm{~min}$ and an $\alpha$-energy of $7.33 \pm 0.03 \mathrm{MeV}$, while ${ }^{247}$ Es was said to have exactly the same $\alpha$-energy, but a half-life of $5.0 \pm 0.3 \mathrm{~min}$. They were also able to measure the $\alpha$ - to EC- branching ratio for these nuclides, $0.11 \pm 0.02$ and approximately 0.07 for ${ }^{246}$ Es and ${ }^{247}$ Es, respectively. Eskola further refined these values in a later experiment [ESK73]. The half-life of ${ }^{247}$ Es was determined to be $4.7 \pm 0.3 \mathrm{~min}$, and it was also found that ${ }^{246}$ Es and ${ }^{247}$ Es actually have different $\alpha$-energies. ${ }^{246}$ Es was reported as having an $\alpha$-energy of $7.36 \pm 0.03 \dot{\mathrm{MeV} \text { and }}{ }^{247}$ Es was reported as $7.31 \pm 0.03 \mathrm{MeV}$. 
Hatsukawa et al. [HAT89] studied the production of several einsteinium isotopes, from ${ }^{245}$ Es to ${ }^{249}$ Es. ${ }^{246}$ Es through ${ }^{249}$ Es were studied via the ${ }^{249} \mathrm{Cf}(\mathrm{p}, \mathrm{xn}){ }^{250-\mathrm{x}}$ Es reaction. Figure 3.1 shows the excitation functions produced by varying the proton energy from 18 $\mathrm{MeV}$ to $33 \mathrm{MeV}$. They also produced ${ }^{243} \mathrm{Es}$ via the ${ }^{233} \mathrm{U}\left({ }^{14} \mathrm{~N}, 4 \mathrm{n}\right)$ reaction, but did not include an excitation function in the paper since the beam was only run at two energies (78 MeV and $82 \mathrm{MeV}$.) They did include an $\alpha$-energy spectrum from this reaction, and it has proved to be a very valuable resource for our experiments. Another reaction presented in the paper was ${ }^{237} \mathrm{~Np}\left({ }^{12} \mathrm{C}, \mathrm{xn}\right){ }^{249-x} \mathrm{Es}$ at projectile energies ranging from 67 $\mathrm{MeV}$ to $81 \mathrm{MeV}$. An excitation function is given, but only for the production of ${ }^{245} \mathrm{Es}$. In the $\alpha$-energy spectrum from this reaction, ${ }^{244} \mathrm{Cf}$ is clearly observed, along with ${ }^{245} \mathrm{Es}$ and ${ }^{245} \mathrm{Cf}$. ${ }^{244} \mathrm{Cf}$ could either be produced directly in the reaction via the p3n exit channel, or it could be the EC-daughter of ${ }^{244} \mathrm{Es}$. In reactions with heavy projectiles and an actinide target, the $5 n$ exit channels usually have significant production cross sections. Based on the spectrum in [HAT89] and the data presented in [GAN80], we decided to use the ${ }^{237} \mathrm{~Np}\left({ }^{12} \mathrm{C}, 5 \mathrm{n}\right)$ reaction in a search for delayed fission in ${ }^{244} \mathrm{Es}$. This will be discussed in more detail in later chapters. Table 3.2 lists the current known values of half-life and decay energy (or decay mode) for the neutron-deficient einsteinium isotopes from ${ }^{242} \mathrm{Es}$ through ${ }^{249} \mathrm{Es}$, as well as for some of their californium EC-daughters. 
Table 3.2. Decay properties of the known neutron-deficient isotopes of einsteinium and some of their californium EC-daughters.

\begin{tabular}{|c|c|c|c|c|c|}
\hline Nuclide & Half-Life & $\begin{array}{c}\alpha \text {-Branch } \\
(\%)\end{array}$ & $\begin{array}{c}\text { EC-Branch } \\
(\%)\end{array}$ & $\begin{array}{c}\alpha \text {-Energy } \\
(\mathrm{MeV})\end{array}$ & Reference(s) \\
\hline${ }^{242}$ Es & $16_{-4}^{+6} \mathrm{~s}$ & Unknown & Unknown & $7.920 \pm 0.020$ & NIN96 \\
\hline${ }^{243} \mathrm{Es}$ & $21 \pm 2 s$ & $>30$ & $<70$ & $\begin{array}{l}7.899(87 \%) \\
7.939(13 \%)\end{array}$ & FIR96 \\
\hline${ }^{244}$ Es & $37 \mathrm{~s}$ & $\begin{array}{l}0 \\
4\end{array}$ & $\begin{array}{c}100 \\
96\end{array}$ & $\begin{array}{c}\text { N/A } \\
7.57 \pm 0.2\end{array}$ & $\begin{array}{l}\text { ESK70 } \\
\text { FIR96 }\end{array}$ \\
\hline${ }^{245} \mathrm{Es}$ & $1.1 \pm 0.1 \mathrm{~min}$ & 40 & 60 & $\begin{array}{c}7.730(79 \%) \\
7.699(13 \%) \\
7.780(5 \%) \\
7.654(3 \%)\end{array}$ & FIR96 \\
\hline${ }^{246} \mathrm{Es}$ & $7.7 \pm 0.5 \mathrm{~min}$ & 9.9 & 90.1 & 7.360 & FIR96 \\
\hline${ }^{247}$ Es & $\begin{array}{c}4.55 \pm 0.26 \\
\min \end{array}$ & 7 & 93 & $\begin{array}{l}7.323(86 \%) \\
7.275(12 \%)\end{array}$ & FIR96 \\
\hline & & & & $7.213^{-}(2 \%)$ & \\
\hline${ }^{248} \mathrm{Es}$ & $27 \pm 4 \mathrm{~min}$ & 0.25 & 99.75 & 6.870 & FIR96 \\
\hline${ }^{249} \mathrm{Es}$ & $\begin{array}{c}102.2 \pm 0.6 \\
\min \end{array}$ & 0.57 & 99.43 & $\begin{array}{c}6.776(93 \%) \\
6.716(7 \%)\end{array}$ & FIR96 \\
\hline${ }^{242} \mathrm{Cf}$ & $3: 5 \pm 0.1 \mathrm{~min}$ & 100 & 0 & $\begin{array}{l}7.385(80 \%) \\
7.351(20 \%)\end{array}$ & FIR96 \\
\hline${ }^{244} \mathrm{Cf}$ & $\begin{array}{c}19.4 \pm 0.6 \\
\min \end{array}$ & 100 & 0 & $\begin{array}{l}7.213(75 \%) \\
7.176(25 \%) \\
\end{array}$ & FIR96 \\
\hline${ }^{245} \mathrm{Cf}$ & $45 \pm 2 \mathrm{~min}$ & 36 & 64 & $\begin{array}{c}7.137(91 \%) \\
7.084(9 \%)\end{array}$ & FIR96 \\
\hline${ }^{246} \mathrm{Cf}$ & $35.7 \pm 0.5 \mathrm{~h}$ & 100 & 0 & $\begin{array}{l}6.750(79 \%) \\
6.708(21 \%)\end{array}$ & FIR96 \\
\hline
\end{tabular}




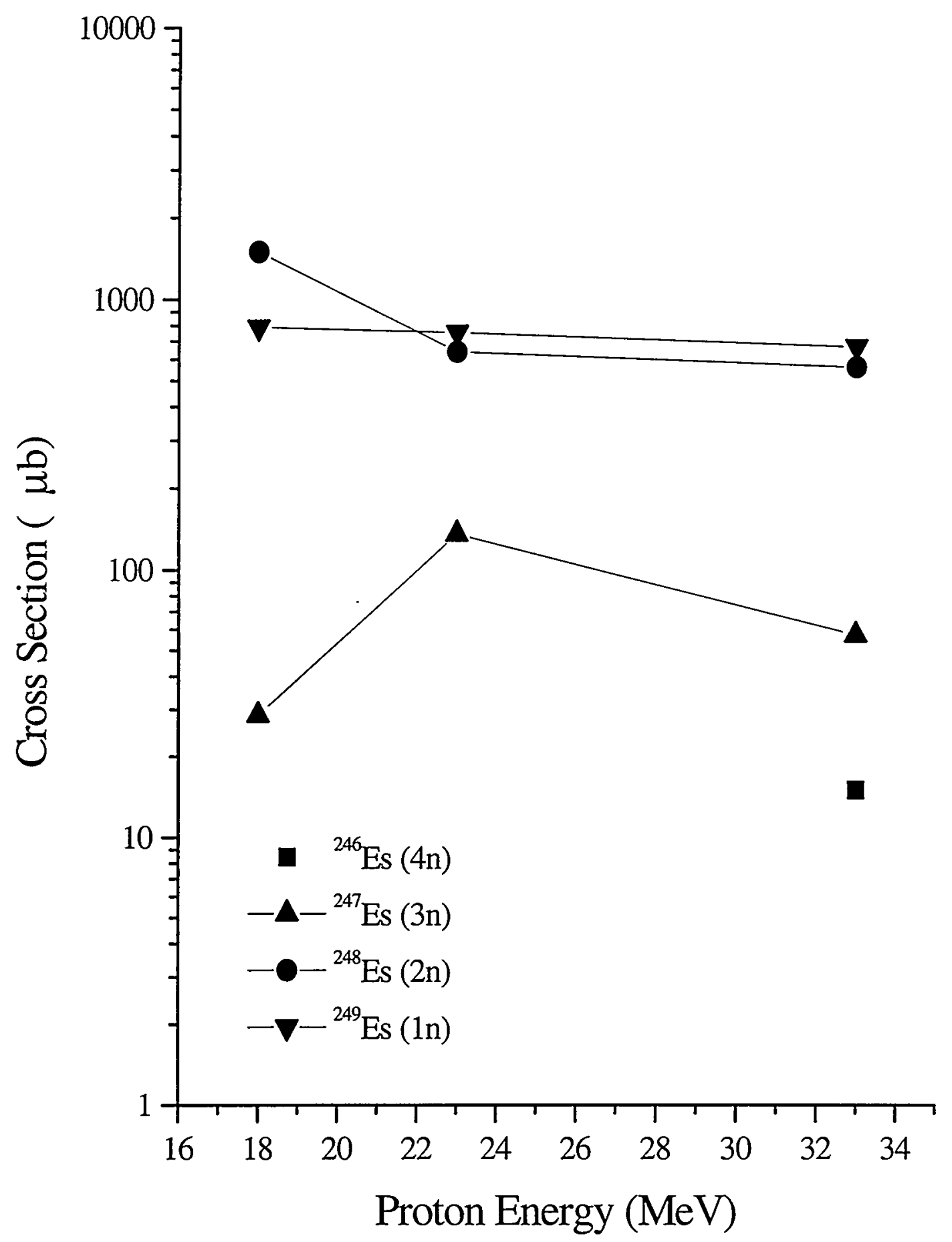

Figure 3.1: Excitation function for the production of einsteinium isotopes via the ${ }^{249} \mathrm{Cf}(\mathrm{p}, \mathrm{xn}){ }^{250-\mathrm{x}}$ Es reaction. Data is from [HAT89]. Only one cross section was given in the text for the production of ${ }^{246} \mathrm{Es}$. 


\section{Targets and Irradiations}

\subsection{Target Configurations}

\subsubsection{Single Target Bombardments}

For the production of lighter einsteinium isotopes, a single, thick actinide target was bombarded with appropriate projectiles. A schematic of the single target arrangement is shown in Figure 4.1. The target system is contained in a glove box at the end of one of the beam lines coming from the 88-Inch Cyclotron at Lawrence Berkeley National Laboratory (LBNL). The beam enters the back of the target system through a 6$\mathrm{mm}$ graphite collimator and then passes through a $1.8-\mathrm{mg} / \mathrm{cm}^{2} \mathrm{HAVAR}$ vacuum window, which is used to contain $\mathrm{N}_{2}$ cooling gas between this foil and the $2.32-\mathrm{mg} / \mathrm{cm}^{2}$ Be target backing foil. After passing through the cooling gas and target backing, the beam enters the target. The collimator and graphite beam stop are both water cooled.

After recoiling from the target, reaction products are collected using a $\mathrm{He} / \mathrm{KCl}$ gas-jet transport system. He gas flows over $\mathrm{KCl}$ crystals that are heated in a $640^{\circ} \mathrm{C}$ oven to form aerosols. The $\mathrm{He}$ gas and $\mathrm{KCl}$ aerosols enter the target chamber via a capillary at a flow rate of $2.5 \mathrm{~L} / \mathrm{min}$. The reaction products recoil into the target chamber and are thermalized in the He, which is kept at a pressure of $1.2 \mathrm{~atm}$. They become attached to the $\mathrm{KCl}$ aerosols, and are swept out of the target system through a 1.4-mm i. d. Teflon capillary. The capillary delivers the activity to our detection system, which will be described in Chapter 5. The yield of this transport system was determined to be $60 \pm 20 \%$ based on previous experiments [WIL97], and the transport time from the target 


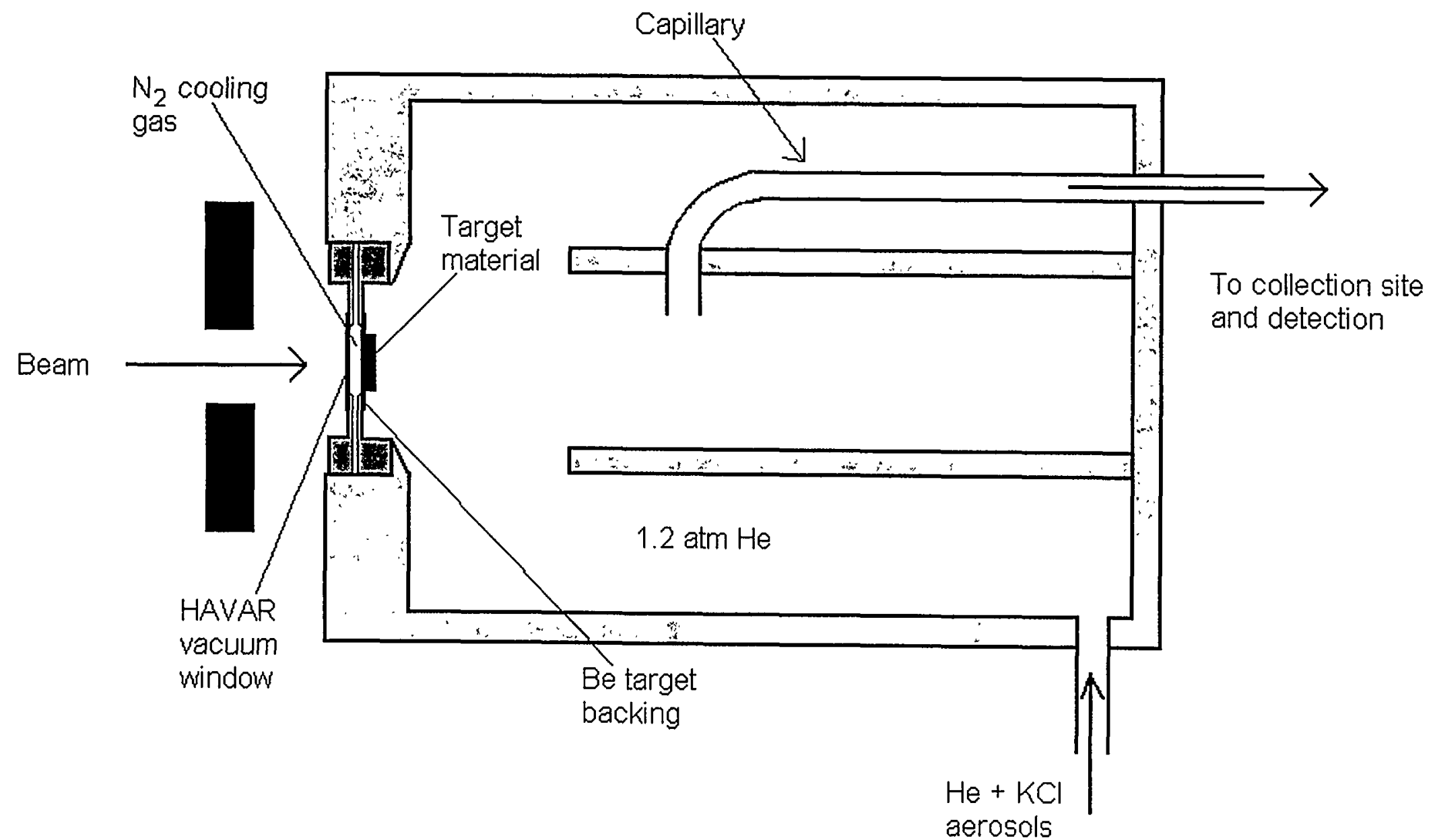

Figure 4.1: Illustration of the target recoil chamber used in irradiations of a single, thick actinide target. 
chamber to the collection site was $1.0 \pm 0.3 \mathrm{~s}$ [LAN98].

\subsubsection{Multiple Target Bombardments}

The cross sections for producing heavier isotopes of einsteinium in reactions with an actinide target and heavy projectiles are very small, leading to extremely low production rates. In order to study the properties of these isotopes, it was necessary to use reactions with light projectiles instead. These reactions have much larger cross sections, but because of the smaller mass of the light projectile, the recoil momentum transferred to the compound nucleus is also much smaller. This can be seen in the following conservation of momentum relationship,

$$
p_{\text {proj. }}=p_{\mathrm{CN}}
$$

where $p$ is the particle momentum, the subscript proj. stands for projectile and $\mathrm{CN}$ stands for compound nucleus. The momentum and energy of a particle are related by the relationship

$$
p_{i}^{2}=2 m_{i} E_{i}
$$

where $m$ is the mass of the particle under consideration and $E$ is the kinetic energy (assuming a nonrelativistic system.) Substituting this into equation 4.1 results in the following conservation relationship for the projectile and compound nucleus:

$$
m_{\text {proj. }} E_{\text {proj. }}=m_{\mathrm{CN}} E_{\mathrm{CN}}
$$

in the laboratory frame of reference $\left(p_{\text {target }}=0\right.$. $)$ Based on the above relationship, the compound nucleus would have very little recoil energy from a light beam unless very

high incident energies were used. As an example, consider the reaction of a ${ }^{249} \mathrm{Cf}$ target bombarded with $40-\mathrm{MeV}$ protons. Using equation 4.3 , the product of the mass and 
energy of the projectile is $40 \mathrm{MeV}$. Dividing this quantity by the mass of the CN (250) results in a recoil energy of only $0.16 \mathrm{MeV}$ for the ${ }^{250} \mathrm{Cf} \mathrm{CN}$. The effective thickness of the target is limited to approximately the recoil range of the $\mathrm{CN}$ in the target material. The recoil range of an einsteinium compound nucleus in a typical actinide oxide target is a few tens of micrograms per square centimeter [NOR70].

The Light Ion Multiple Target system (LIM) was developed in order to maximize the large production rates associated with light projectile bombardments, without being restricted by the small recoil energies of the CN [HAL89A]. The LIM system allows for the simultaneous bombardment of up to 23 targets by a single beam. The effective target thickness of a single, thick target is only on the order of $10-30 \mu \mathrm{g} / \mathrm{cm}^{2}$. The LIM system therefore increases production rates by using many targets simultaneously instead of being limited by the small recoil range of only one target. A schematic of the LIM system is shown in Figure 4.2.

Each target backing is attached to an aluminum target holder (also seen in Figure 4.2) with epoxy adhesive. The $\mathrm{He} / \mathrm{KCl}$ gas-jet described in the previous section enters the LIM target chamber via a capillary as in the single target configuration. The slots on the side of each target holder are alternated so that the $\mathrm{He} / \mathrm{KCl}$ aerosol mixture is forced to sweep between all of the holders, thereby collecting reaction products from each target. The gas-jet then exits the chamber via a capillary positioned at the opposite end, and activity is delivered to a detection system as described previously. The graphite beam stop is in a water-cooled copper jacket, which dissipates heat generated by the beam passing through the system. The overall gas-jet efficiency of the LIM system was measured to be $50-95 \%$ in previous experiments [HAL89]. 
Light Ion Multiple (LIM)

Target System
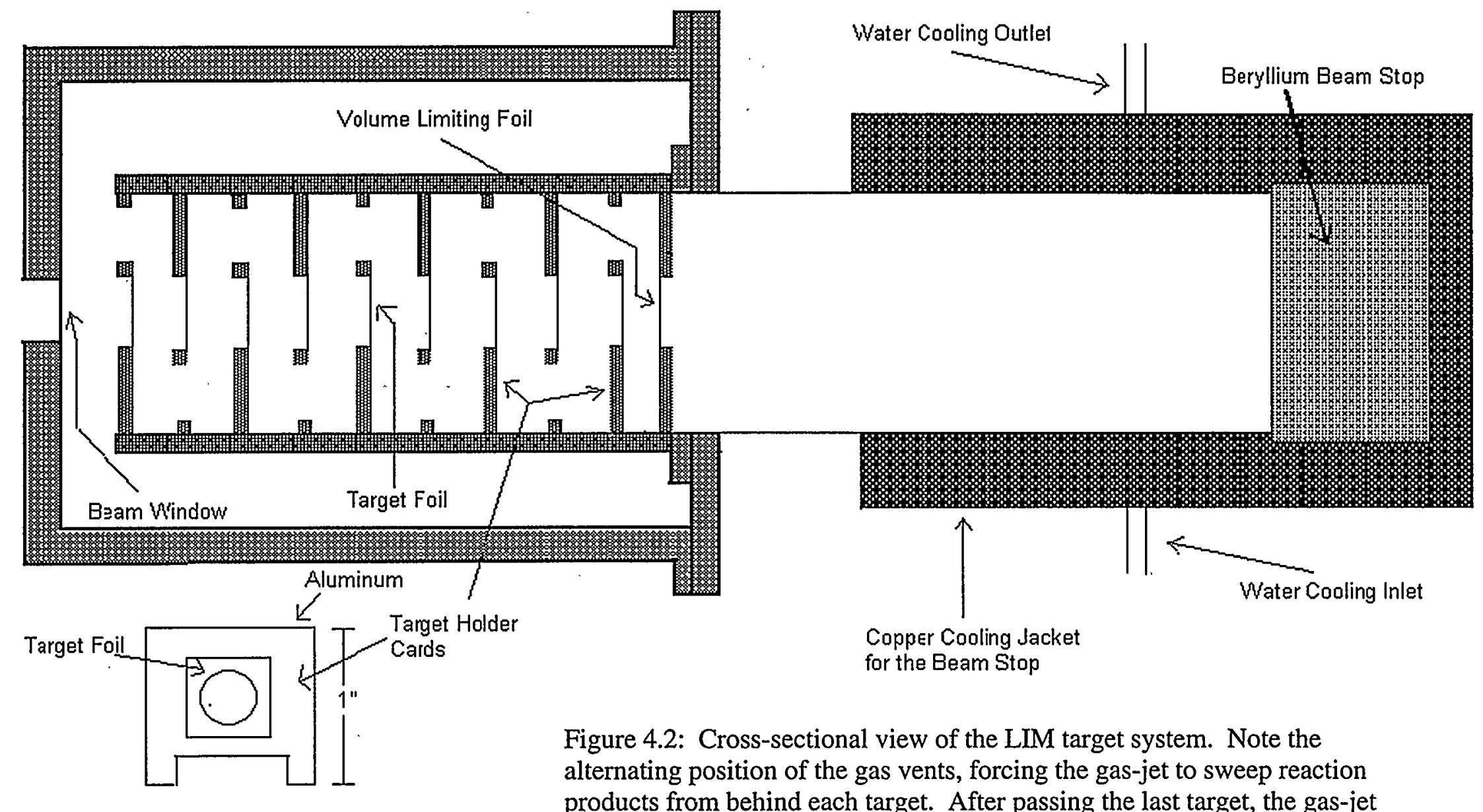

Figure 4.2: Cross-sectional view of the LIM target system. Note the alternating position of the gas vents, forcing the gas-jet to sweep reaction products from behind each target. After passing the last target, the gas-jet is removed via a capillary. 
Fission products produced during beam irradiations have much larger recoil ranges than the $\mathrm{CN}$. The targets in the LIM system are placed such that the spacing is larger than the range of the $\mathrm{CN}$ in $\mathrm{He}$, but less than the range of typical fission fragments. This causes fission products to become embedded in the back of the next target, while the $\mathrm{CN}$ evaporation residues, which have much shorter ranges in He, are stopped in the gas, become attached to aerosols and are swept out of the chamber with the gas-jet. The recoil ranges in $\mathrm{He}$ of einsteinium compound nuclei produced in our experiments are on the order of $1 \mathrm{~mm}$, and typical fission fragments have ranges that are approximately 100 times larger [NOR70]. For irradiation of ${ }^{249} \mathrm{Cf}$ targets, the LIM was setup with $19{ }^{249} \mathrm{Cf}$ targets (these targets are described later.) A volume limiting foil of 1-mil $\left(4.7 \mathrm{mg} / \mathrm{cm}^{2}\right)$ Be was placed at the end of the LIM chamber. A 1-mil Be vacuum window was used to contain the He gas in the recoil chamber. Each of the targets was spaced approximately 3.2-mm apart.

\subsection{Target Preparation}

\subsubsection{Uranium Targets}

The ${ }^{233} \mathrm{U}$ targets used for the production of einsteinium isotopes were produced by recycling old uranium targets. Two $\mathrm{U}_{3} \mathrm{O}_{8}$ targets $(700 \mu \mathrm{g}$ and $271 \mu \mathrm{g})$ on beryllium backings were dissolved in ultra pure concentrated $\mathrm{HCl}$ (lead and heavy metal concentration less than one part per million) until the target material and backings had completely dissolved. The uranium solution was then sorbed on a $1-\mathrm{cm}$ by $9-\mathrm{cm}$ anion exchange column (AG 1-X8 resin, 200 mesh) which was then rinsed with several free column volumes (FCV) of the same high grade concentrated $\mathrm{HCl}$ to remove lead and 
other contaminants [GIN62]. The uranium was then eluted from the column with $0.1 \mathrm{M}$ $\mathrm{HCl}$ (made from high grade $\mathrm{HCl}$ ) and several FCV's were collected. A $10-\mu \mathrm{L}$ aliquot was taken from the resulting yellow solution, dried on a platinum disk and assayed using a low geometry surface barrier detector. The uranium solution was found to contain approximately $260 \mu \mathrm{g}$ of ${ }^{233} \mathrm{U}$. The solution was dried and then dissolved in $0.5 \mathrm{~mL}$ of isopropyl alcohol (IPA) to yield a solution that was approximately $0.5 \mathrm{mg} / \mathrm{mL}$ in ${ }^{233} \mathrm{U}$. The targets were prepared by electrodeposition [AUM74], [MUL75] on 0.5-mil $\left(2.32 \mathrm{mg} / \mathrm{cm}^{2}\right)$ Be foil. Beryllium was chosen for the target backings for several reasons. It is mechanically strong, and can withstand large beam intensities, as well as large amounts of heat produced as the beam passes through the foil. Also, less background radiation is produced from interactions between the beam and beryllium foil than in other metals such as molybdenum [HAL89]. Figure 4.3 shows the electroplating cell used for target preparation. Several layers of ${ }^{233} \mathrm{U}$ were plated onto a single backing to produce each target. Each layer was made by adding $50 \mu \mathrm{L}$ of the ${ }^{233} \mathrm{U}$ stock solution and $1 \mathrm{~mL}$ of clean IPA to the plating cell. The uranium was electroplated from the IPA onto the Be backing at 500 volts $(0.6 \mathrm{~mA})$ for 30 minutes. The layers were baked in a $500^{\circ} \mathrm{C}$ oven for 20 minutes to convert the uranium to the oxide. The target area was $0.28 \mathrm{~cm}^{2}$.

The thickness of the targets was determined by $\alpha$-analysis using a low geometry surface barrier detector with a 3/8-inch collimator operated in He and a detection efficiency of $6.44 \times 10^{-3}$. During the $\alpha$-analysis the number of ${ }^{233} \mathrm{U} \alpha$-particles detected per unit time is divided by the efficiency of the detector, resulting in the activity (A) of the ${ }^{233} \mathrm{U}$ plated on the target, where $\mathrm{A}=\lambda \mathrm{N}$ in disintegrations per minute and $\mathrm{N}$ is the number of ${ }^{233} U$ nuclei. $N$ is then equivalent to the weight of the plated ${ }^{233} U$ in grams 


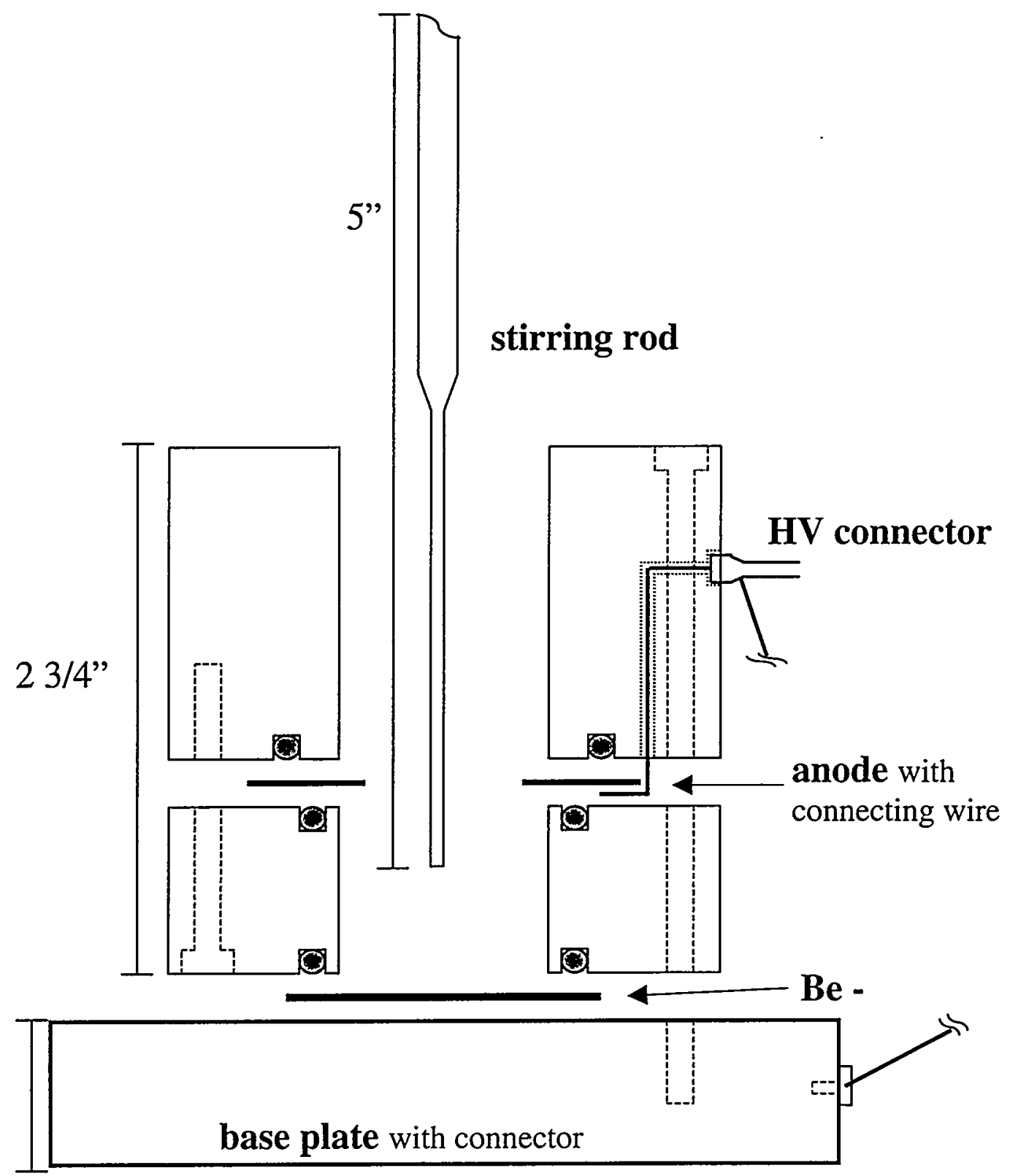

Figure 4.3: Schematic of the electroplating cell used for target production. The stock solution and IPA are added to the center where the stirring rod is positioned. The area of the opening (and the resulting target) is $0.28 \mathrm{~cm}^{2}$. 
when the atomic mass $(233 \mathrm{~g} / \mathrm{mol})$ and the number of atoms per mole $\left(6.02 \times 10^{23}\right.$ atoms $/ \mathrm{mol}$ ) are taken into account. Two targets were prepared from the ${ }^{233} \mathrm{U}$ stock solution. Target 233U95A was $502 \mu \mathrm{g} / \mathrm{cm}^{2}$ and target 233U95B was $395 \mu \mathrm{g} / \mathrm{cm}^{2}$. Target 233U95A was used in all experiments requiring a ${ }^{233} \mathrm{U}$ target.

\subsubsection{Neptunium Targets}

$1.61 \mathrm{mg}$ of ${ }^{237} \mathrm{~Np}$ in an aqueous solution were evaporated to dryness. The remaining ${ }^{237} \mathrm{~Np}$ was dissolved in a few drops of ultra pure grade concentrated $\mathrm{HCl}$ and again evaporated to dryness. This process was repeated a few times to make sure that the neptunium was in the chloride form for the chemical separation that followed. The resulting green salt was dissolved in concentrated $\mathrm{HCl}$ using heat to ensure a complete dissolution. The neptunium solution was sorbed on a $7.5-\mathrm{mm}$ by $27.5-\mathrm{mm}$ anion exchange column (AG 1X-8 resin, 200-400 mesh) which was then rinsed with several FCV's of concentrated $\mathrm{HCl}$. Neptunium, plutonium, uranium and protactinium sorb on the column, while lead and other impurities are removed in the $\mathrm{HCl}$ wash [HAL89]. Plutonium was then removed via elution with a $7: 1$ solution of concentrated $\mathrm{HCl}: \mathrm{HI}$. The column was washed again with concentrated $\mathrm{HCl}$ to remove any residual $\mathrm{HI}$. Finally, the neptunium was eluted from the column with $2 \mathrm{M} \mathrm{HCl}$ (prepared with high grade $\mathrm{HCl}$.) A $10-\mu \mathrm{L}$ assay of the eluant was counted with a surface barrier detector operated under vacuum with a detection efficiency of approximately $30 \%$. The solution was found to contain $480 \mu \mathrm{g}$ of ${ }^{237} \mathrm{~Np}$ and $0.11 \mu \mathrm{g}$ total of both ${ }^{238} \mathrm{Pu}$ and ${ }^{239} \mathrm{Pu}$.

The resulting neptunium solution was evaporated to dryness. One $\mathrm{mL}$ of concentrated $\mathrm{HNO}_{3}$ was added to the salt, the solution was evaporated, and this process 
was repeated to convert the neptunium to the nitrate form. The resulting solid was dissolved in $1 \mathrm{~mL}$ of IPA to make a solution that was approximately $0.5 \mathrm{mg} / \mathrm{mL}$ in ${ }^{237} \mathrm{~Np}$.

Two targets were prepared by electrodeposition on 0.5 -mil Be foil. Each target consisted of several layers of ${ }^{237} \mathrm{~Np}$ plated onto a single Be backing foil. Each layer was made by adding $50 \mu \mathrm{L}$ of the stock solution and $1.25 \mathrm{~mL}$ of IPA to the plating cell. The neptunium was electroplated from the IPA onto the Be backing at 300 volts $(0.7 \mathrm{~mA})$ for 30 minutes. After electroplating, each layer was baked in a $450^{\circ} \mathrm{C}$ oven for 30 minutes to convert the neptunium to the oxide form. The target area was $0.28 \mathrm{~cm}^{2}$.

The thickness of the targets was determined by $\alpha$-analysis using a surface barrier detector operated under vacuum with a detection efficiency of $34 \%$ as described in section 4.2.1. Two ${ }^{237} \mathrm{~Np}$ targets were prepared from the stock solution. Target 237NPA was $1332 \mu \mathrm{g} / \mathrm{cm}^{2}$ and target $237 \mathrm{NPB}$ was $487.5 \mu \mathrm{g} / \mathrm{cm}^{2}$. Target $237 \mathrm{NPB}$ was used in all experiments requiring a ${ }^{237} \mathrm{~Np}$ target.

\subsubsection{Californium Targets}

Several thin ${ }^{249} \mathrm{Cf}$ targets were also prepared by electrodeposition for use in the LIM system. Each target consisted of only one layer of ${ }^{249} \mathrm{Cf}$ plated on a 1-mil (4.7$\mathrm{mg} / \mathrm{cm}^{2}$ ) Be foil. Thicker Be foils were used for these targets because the energy lost by proton beams through $\mathrm{Be}$ is negligible [NOR70]. The source of the ${ }^{249} \mathrm{Cf}$ was an old

${ }^{249} \mathrm{Bk}$ target that had originally contained $280 \mu \mathrm{g}$ of ${ }^{249} \mathrm{Bk} .{ }^{249} \mathrm{Bk}$ undergoes beta-decay to ${ }^{249} \mathrm{Cf}$ with a $320-\mathrm{d}$ half-life. This target was over eight years old, which meant that almost all of the berkelium had been transformed into ${ }^{249} \mathrm{Cf}$.

The old target was dissolved in $10 \mathrm{M} \mathrm{HNO}_{3}$ using heat to completely dissolve the 
Be backing. Any residual berkelium was removed from the solution by performing an extraction using di (2-ethyl hexyl) orthophosphoric acid (HDEHP.) The structure of HDEHP is shown below:<smiles>CCC(CC)COP(=O)(O)OCC(CC)CC</smiles>

This complexing agent extracts $3+$ and higher oxidation states from dilute acid solutions, and 4+ and higher oxidation states from concentrated acid solutions [HIG60], [PEP57]. Berkelium can exhibit both $3+$ and $4+$ oxidation states while californium only has a $3+$ state. Saturated $\mathrm{KBrO}_{3}$ was added to the $\mathrm{Bk} / \mathrm{Cf}$ solution to oxidize the berkelium to the $4+$ state. The $\mathrm{Bk}^{4+}$ was then extracted into 0.5 M HDEEIP in $\mathrm{n}$-heptane and the $\mathrm{Cf}^{3+}$ remained in the aqueous phase. A flow chart of the entire extraction procedure is shown in Figure 4.4. The resulting californium solution was dried and dissolved in $0.5 \mathrm{~mL}$ of IPA to yield a solution that was approximately $0.3 \mathrm{mg} / \mathrm{mL}$ in ${ }^{249} \mathrm{Cf}$.

Each target was made by adding $25 \mu \mathrm{l}$ of the ${ }^{249} \mathrm{Cf}$ stock solution and $1 \mathrm{~mL}$ of clean IPA to the plating cell, and then plating at 600 volts $(0.5 \mathrm{~mA})$ for 30 minutes. The targets were baked in a $500^{\circ} \mathrm{C}$ oven for 20 minutes to convert the californium to the oxide form. The thickness of each target was determined by $\alpha$-analysis using a low geometry surface barrier detector with a 3/8-inch collimator operated in He gas as described in section 4.2.1. The detection efficiency was $1.44 \times 10^{-4}$. Table 4.1 lists the thickness of each of the ${ }^{249} \mathrm{Cf}$ targets. 


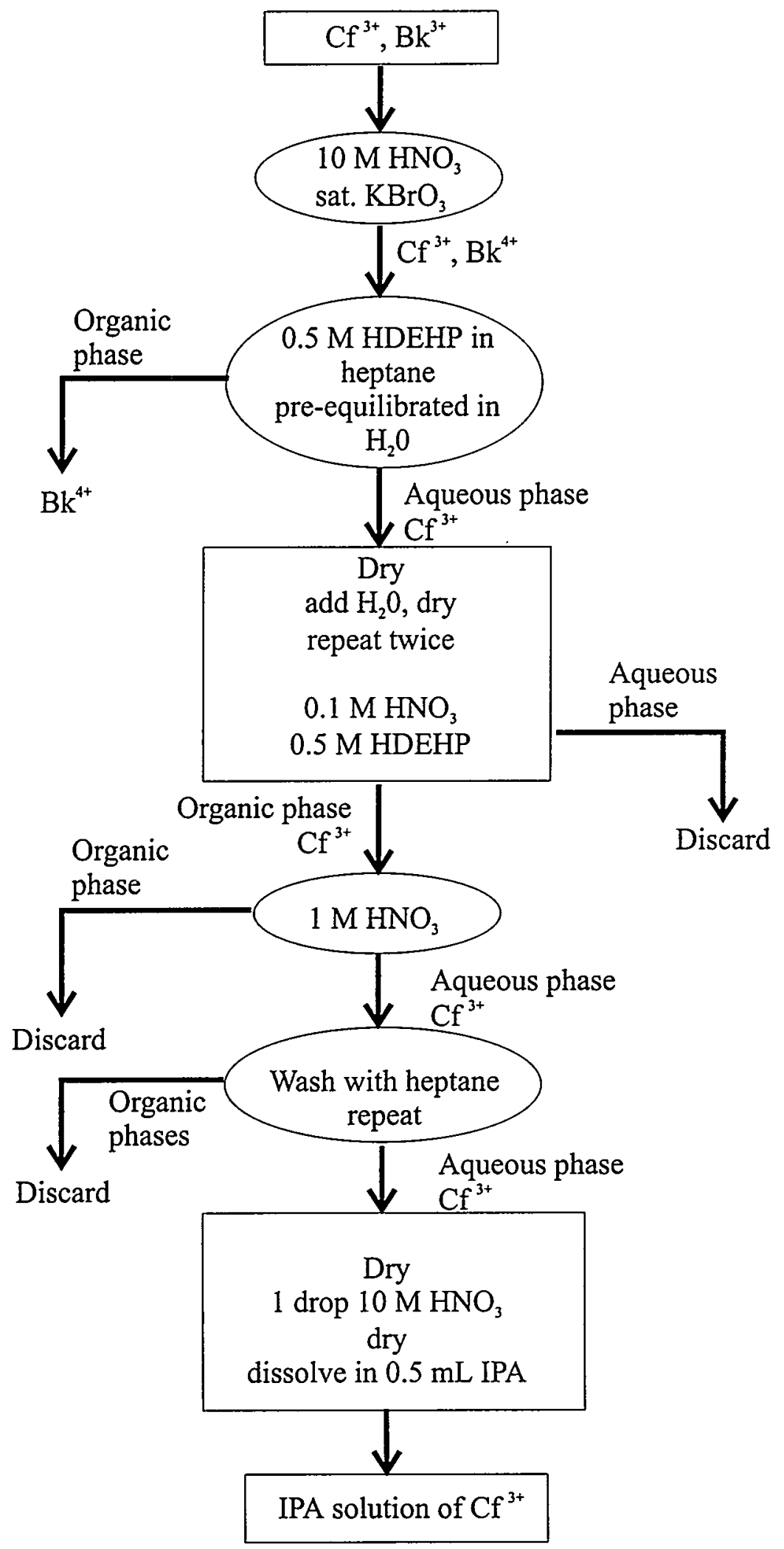

Figure 4.4: Flow chart of the HDEHP extraction procedure used to separate and purify californium from a mixed solution of californium and berkelium. 
Table 4.1. Thickness of the ${ }^{249} \mathrm{Cf}$ targets as determined by low geometry $\alpha$-analysis described in section 4.2.1.

\begin{tabular}{|ccc|}
\hline Target & Activity $(\mathrm{cpm})$ & Thickness $\left(\mu \mathrm{g} / \mathrm{cm}^{2}\right)$ \\
\hline 249 Cf1 & 6295 & 6.0 \\
249 Cf2 & 13219 & 12.6 \\
249 Cf3 & 11016 & 10.5 \\
249 Cf 4 & 11331 & 10.8 \\
249 Cf5 & 14373 & 13.7 \\
249 Cf6 & 15737 & 15.0 \\
249 Cf7 & 14059 & 13.4 \\
249 Cf8 & 15528 & 14.8 \\
249 Cf9 & 13743 & 13.1 \\
249 Cf10 & 13954 & 13.3 \\
249 Cf11 & 15318 & 14.6 \\
249 Cf12 & 18465 & 17.6 \\
249 Cf13 & 17836 & 17.0 \\
249 Cf14 & 15003 & 14.3 \\
249 Cf15 & 17836 & 17.0 \\
249 Cf16 & 16787 & 16.0 \\
249 Cf17 & 20668 & 19.7 \\
249 Cf1 18 & 19829 & 18.9 \\
249 Cf1 & 12905 & 12.3 \\
\hline
\end{tabular}

\subsection{Irradiations}

All projectiles were provided by the LBNL 88-Inch Cyclotron. Beam energies in this dissertation refer to the laboratory frame of reference. With a single target configuration (section 4.1.1) the beam loses energy after exiting the cyclotron as it passes through the HAVAR vacuum window, $\mathrm{N}_{2}$ cooling gas and Be target backing. Beam energies for single target experiments refer to the energy of the beam after passing through the Be before entering the target material. Corrections for beam energy loss have been taken into account [NOR70]. For the LIM target configuration the energies of proton beams from the cyclotron are equivalent to the energies of the beams after passing through the Be vacuum window and the first Be target backing (section 4.1.2) [NOR70].

The excitation function of the ${ }^{233} \mathrm{U}\left({ }^{14} \mathrm{~N}, \mathrm{xn}\right){ }^{247-\mathrm{x}} \mathrm{Es}$ reaction was determined by 
bombarding ${ }^{233} \mathrm{U}$ with projectiles at four different energies $(80 \mathrm{MeV}, 87 \mathrm{MeV}, 93 \mathrm{MeV}$ and $100 \mathrm{MeV}$ ) at an intensity of 3.0 e $\mu \mathrm{A}$ each time. To study delayed fission properties of ${ }^{242} \mathrm{Es}$, the ${ }^{233} \mathrm{U}\left({ }^{14} \mathrm{~N}, 5 \mathrm{n}\right)$ reaction was used. The ${ }^{14} \mathrm{~N}^{4+}$ beam was originally $84 \mathrm{MeV}(2.8$ $\mathrm{e} \mu \mathrm{A}$ ), corresponding to the maximum of the $5 \mathrm{n}$ exit channel according to the SPIT fusion-evaporation code [SIK67]. SPIT is a computer code that predicts excitation functions. As discussed in Chapter 6, a large amount of ${ }^{243}$ Es was also produced in this reaction, interfering with the detection of ${ }^{242}$ Es. The beam was shifted higher to $89 \mathrm{MeV}$ $(2.3 \mathrm{e} \mu \mathrm{A})$ to try and suppress ${ }^{243} \mathrm{Es}$ production while running as close to the maximum of the excitation function as possible. Figure 4.5 shows the cross sections predicted by the SPIT code for this reaction. Based on the results of the excitation function experiment (see Chapter 6), it was determined that $87 \mathrm{MeV}$ was the best beam energy for maximizing ${ }^{242}$ Es production for the study of ECDF. A final delayed fission experiment was performed at an energy of $87 \mathrm{MeV}$ (3.0 e $\mu$ a.)

Two different reactions were used to study delayed fission in ${ }^{244}$ Es. Initially, the ${ }^{233} \mathrm{U}\left({ }^{15} \mathrm{~N}, 4 \mathrm{n}\right)$ reaction was used. The ${ }^{15} \mathrm{~N}^{4+}$ beam was originally $81 \mathrm{MeV}(2.0 \mathrm{e} \mu \mathrm{A})$ and was later shifted to $86 \mathrm{MeV}(2.2 \mathrm{e} \mu \mathrm{A})$ to increase production of ${ }^{244}$ Es. Figure 4.6 shows the predictions made by SPIT [SIK67] for this reaction. We then performed an excitation function experiment for the ${ }^{233} U\left({ }^{15} \mathrm{~N}, \mathrm{xn}\right){ }^{248-\mathrm{x}}$ Es reaction. During this experiment, the beam was run at $80 \mathrm{MeV}, 85 \mathrm{MeV}, 93 \mathrm{MeV}$ and $100 \mathrm{MeV}$ (3.0 e $\mu \mathrm{A}$ each time.) Based on $\alpha$-energy spectra [HAT89] and SPIT predictions (Figure 4.7), we then decided to try the ${ }^{237} \mathrm{~Np}\left({ }^{12} \mathrm{C}, 5 \mathrm{n}\right)$ reaction to study ECDF of ${ }^{244} \mathrm{Es}$. The ${ }^{12} \mathrm{C}^{4+}$ beam was $81 \mathrm{MeV}$ at an intensity of $3.0 \mathrm{e} \mu \mathrm{A}$. See Chapter 6 for a complete discussion of these experiments.

To study heavier einsteinium isotopes, the ${ }^{249} \mathrm{Cf}(\mathrm{p}, \mathrm{xn})^{250-\mathrm{x}}$ Es reaction was used 
with 19 thin ${ }^{249} \mathrm{Cf}$ targets in the LIM system. We first studied delayed fission in ${ }^{246} \mathrm{Es}$ and ${ }^{248}$ Es using proton beams of $37 \mathrm{MeV}(5.0 \mathrm{e} \mu \mathrm{A})$ and $18 \mathrm{MeV}(3.0 \mathrm{e} \mu \mathrm{A})$, respectively. The SPIT predictions for this reaction are shown in Figure 4.8. We also ran an excitation function of this reaction using $21 \mathrm{MeV}, 28 \mathrm{MeV}, 35 \mathrm{MeV}$ and $40 \mathrm{MeV}$ beams (5.0 e $\mu \mathrm{A}$ each). A $21-\mathrm{MeV}$ proton beam was also used to irradiate a single ${ }^{249} \mathrm{Cf}$ target. A gold catcher foil was located directly behind the ${ }^{249} \mathrm{Cf}$ target to catch all of the reaction products recoiling out of the target. This was used to determine the efficiency of the LIM gas-jet transport system. The results of all experiments will be discussed in Chapter 6 .

Table 4.2 gives a summary of the reactions and beam energies used for the experiments discussed in this dissertation.

Table 4.2. Reactions and projectile energies used for the study of einsteinium isotopes.

\begin{tabular}{|c|c|c|c|c|}
\hline Experiment & Reaction & $\begin{array}{c}\text { Coulomb Barrier } \\
(\mathrm{MeV})^{\mathrm{a}}\end{array}$ & Energy $(\mathrm{MeV})$ & $\begin{array}{c}\text { Intensity } \\
(\mathrm{e} \mu \mathrm{A})\end{array}$ \\
\hline ECDF of ${ }^{242} \mathrm{Es}$ & ${ }^{233} U\left({ }^{14} N, 5 n\right)$ & 75.2 & $\overline{84}$ & 2.8 \\
\hline $\mathrm{ECDF}$ of ${ }^{242} \mathrm{Es}$ & ${ }^{233} \mathrm{U}\left({ }^{14} \mathrm{~N}, 5 \mathrm{n}\right)$ & 75.2 & 87 & 3.0 \\
\hline $\mathrm{ECDF}$ of ${ }^{242} \mathrm{Es}$ & ${ }^{233} \mathrm{U}\left({ }^{14} \mathrm{~N}, 5 \mathrm{n}\right)$ & 75.2 & 89 & 2.3 \\
\hline Excitation Function & ${ }^{233} \mathrm{U}\left({ }^{14} \mathrm{~N}, \mathrm{xn}\right)$ & 75.2 & 80 & 3.0 \\
\hline Excitation Function & ${ }^{233} \mathrm{U}\left({ }^{14} \mathrm{~N}, \mathrm{xn}\right)$ & 75.2 & 87 & 3.0 \\
\hline Excitation Function & ${ }^{233} \mathrm{U}\left({ }^{14} \mathrm{~N}, \mathrm{xn}\right)$ & 75.2 & 93 & 3.0 \\
\hline Excitation Function & ${ }^{233} \mathrm{U}\left({ }^{14} \mathrm{~N}, \mathrm{xn}\right)$ & 75.2 & 100 & 3.0 \\
\hline $\mathrm{ECDF}$ of ${ }^{244} \mathrm{Es}$ & ${ }^{233} U\left({ }^{15} N, 4 n\right)$ & 74.7 & 81 & 2.0 \\
\hline ECDF of ${ }^{244} \mathrm{Es}$ & ${ }^{233} U\left({ }^{15} N, 4 n\right)$ & 74.7 & 86 & 2.2 \\
\hline Excitation Function & ${ }^{233} \mathrm{U}\left({ }^{15} \mathrm{~N}, \mathrm{xn}\right)$ & 74.7 & 80 & 3.0 \\
\hline Excitation Function & ${ }^{233} \mathrm{U}\left({ }^{15} \mathrm{~N}, \mathrm{xn}\right)$ & 74.7 & 85 & 3.0 \\
\hline Excitation Function & ${ }^{233} \mathrm{U}\left({ }^{15} \mathrm{~N}, \mathrm{xn}\right)$ & 74.7 & 93 & 3.0 \\
\hline Excitation Function & ${ }^{233} \mathrm{U}\left({ }^{15} \mathrm{~N}, \mathrm{xn}\right)$ & 74.7 & 100 & 3.0 \\
\hline ECDF of ${ }^{244}$ Es & ${ }^{237} \mathrm{~Np}\left({ }^{12} \mathrm{C}, 5 \mathrm{n}\right)$ & 65.8 & 81 & 3.0 \\
\hline ECDF of ${ }^{246} \mathrm{Es}$ & ${ }^{249} \mathrm{Cf}(\mathrm{p}, 4 \mathrm{n})$ & 13.4 & 37 & 5.0 \\
\hline ECDF of ${ }^{248}$ Es & ${ }^{249} \mathrm{Cf}(p, 2 \mathrm{n})$ & 13.4 & 18 & 3.0 \\
\hline Excitation Function & ${ }^{249} \mathrm{Cf}(\mathrm{p}, \mathrm{xn})$ & 13.4 & 21 & 5.0 \\
\hline Excitation Function & ${ }^{249} \mathrm{Cf}(\mathrm{p}, \mathrm{xn})$ & 13.4 & 28 & 5.0 \\
\hline Excitation Function & ${ }^{249} \mathrm{Cf}(\mathrm{p}, \mathrm{xn})$ & 13.4 & 35 & 5.0 \\
\hline Excitation Function & ${ }^{249} \mathrm{Cf}(\mathrm{p}, \mathrm{xn})$ & 13.4 & 40 & 5.0 \\
\hline $\begin{array}{l}\text { LIM System Yield } \\
\text { Determination }\end{array}$ & ${ }^{249} \mathrm{Cf}(\mathrm{p}, \mathrm{xn})$ & 13.4 & 21 & 5.0 \\
\hline
\end{tabular}

${ }^{a}$ Coulomb barriers were calculated from a variation of equation 1.1 where $V c=1.44\left(Z_{a} Z_{A}\right) /\left(R_{a}+\right.$ $\left.R_{\mathrm{A}}\right) \mathrm{MeV}, \mathrm{R}=\mathrm{RoA}^{1 / 3} \mathrm{fm}$ and $\mathrm{Ro}=1.44$. See section 1.3 for definition of symbols. 


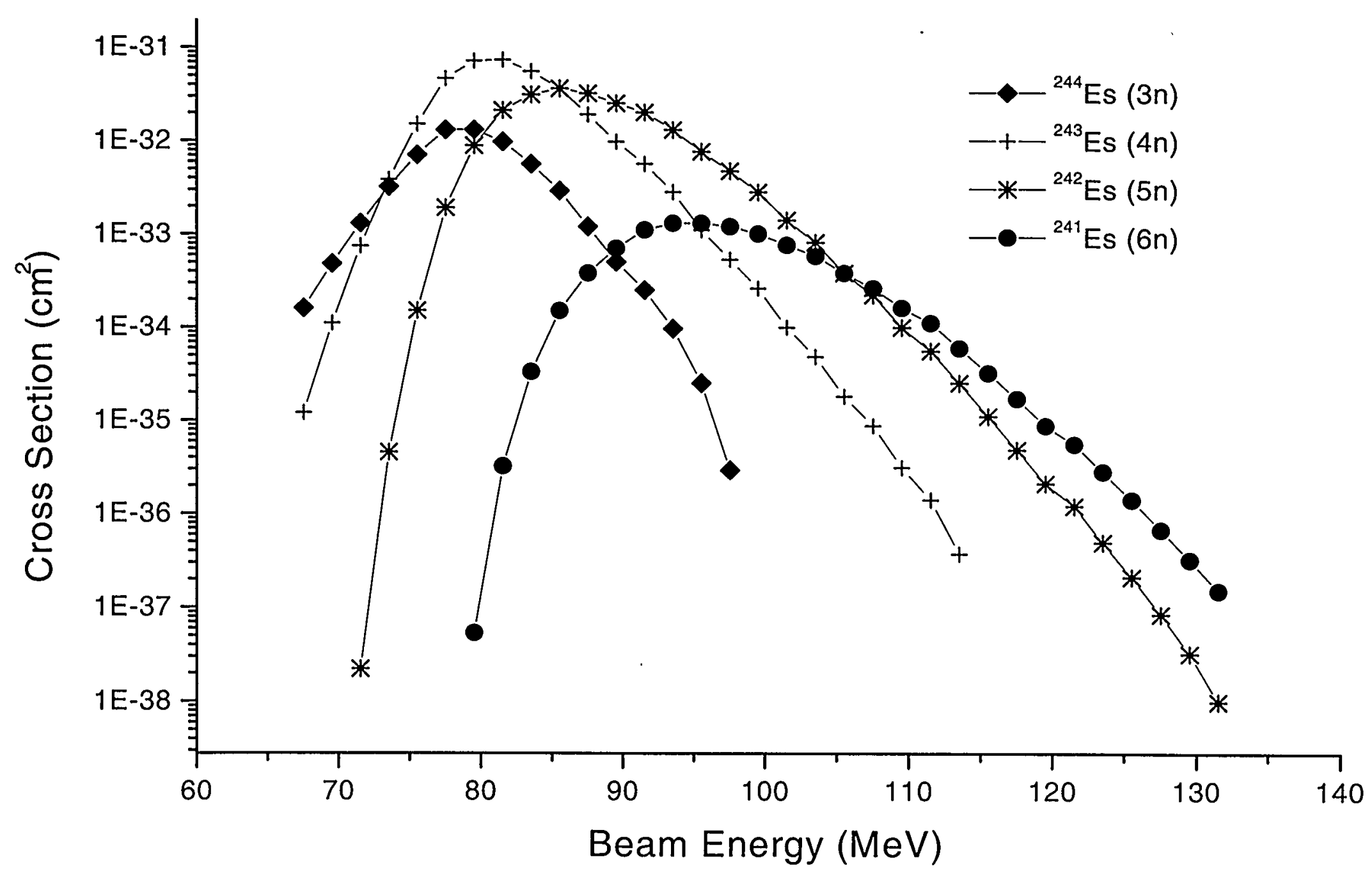

Figure 4.5: Predicted cross sections for the production of einsteinium isotopes using the ${ }^{233} \mathrm{U}\left({ }^{14} \mathrm{~N}, \mathrm{xn}\right){ }^{247-\mathrm{x}}$ Es reaction. Cross sections were determined using the SPIT [SIK67] code. 


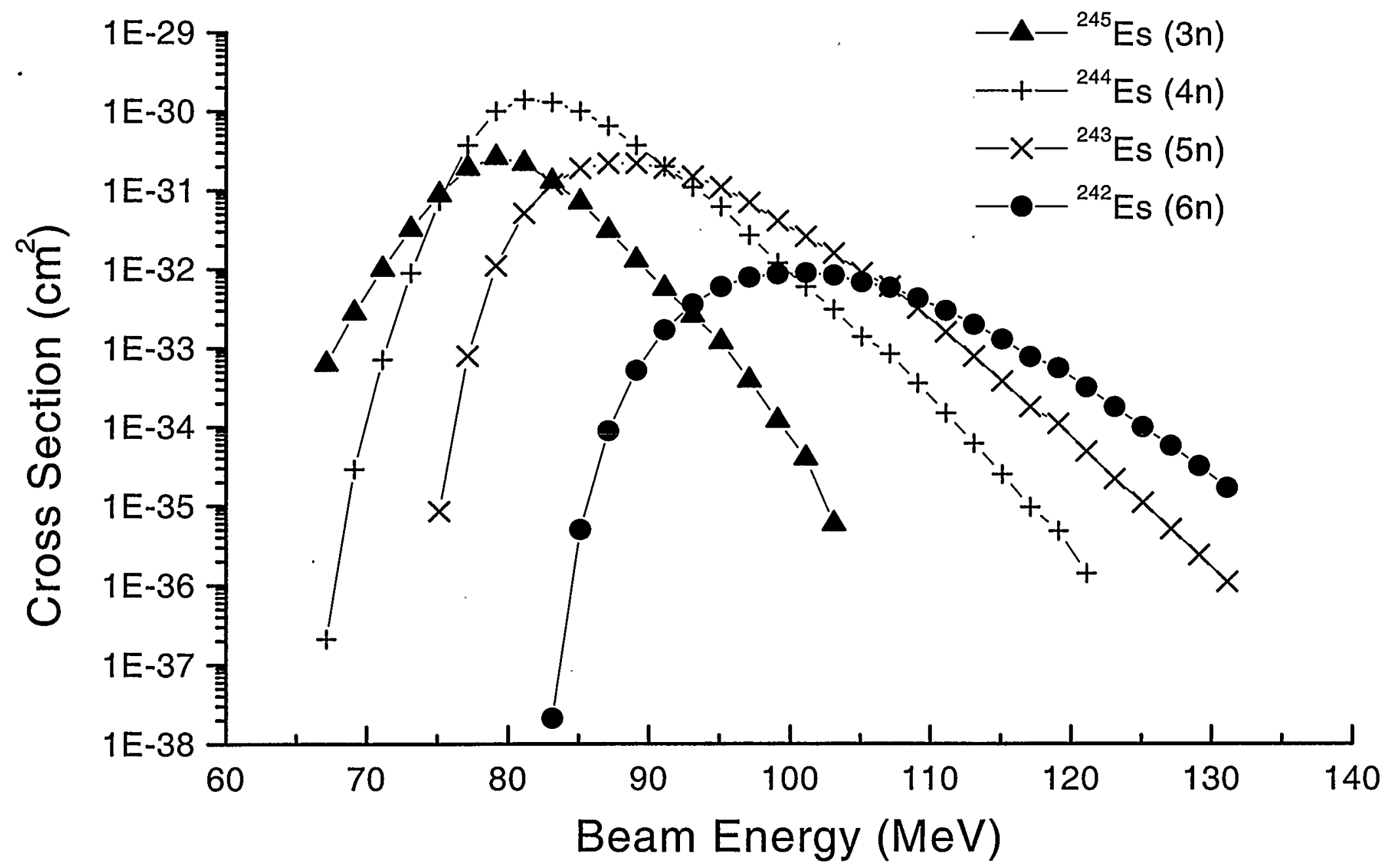

Figure 4.6: Predicted cross sections for the production of einsteinium isotopes using the ${ }^{233} \mathrm{U}\left({ }^{15} \mathrm{~N}, \mathrm{xn}\right){ }^{248-\mathrm{x}} \mathrm{Es}$ reaction. Cross sections were determined using the SPIT [SIK67] code. 


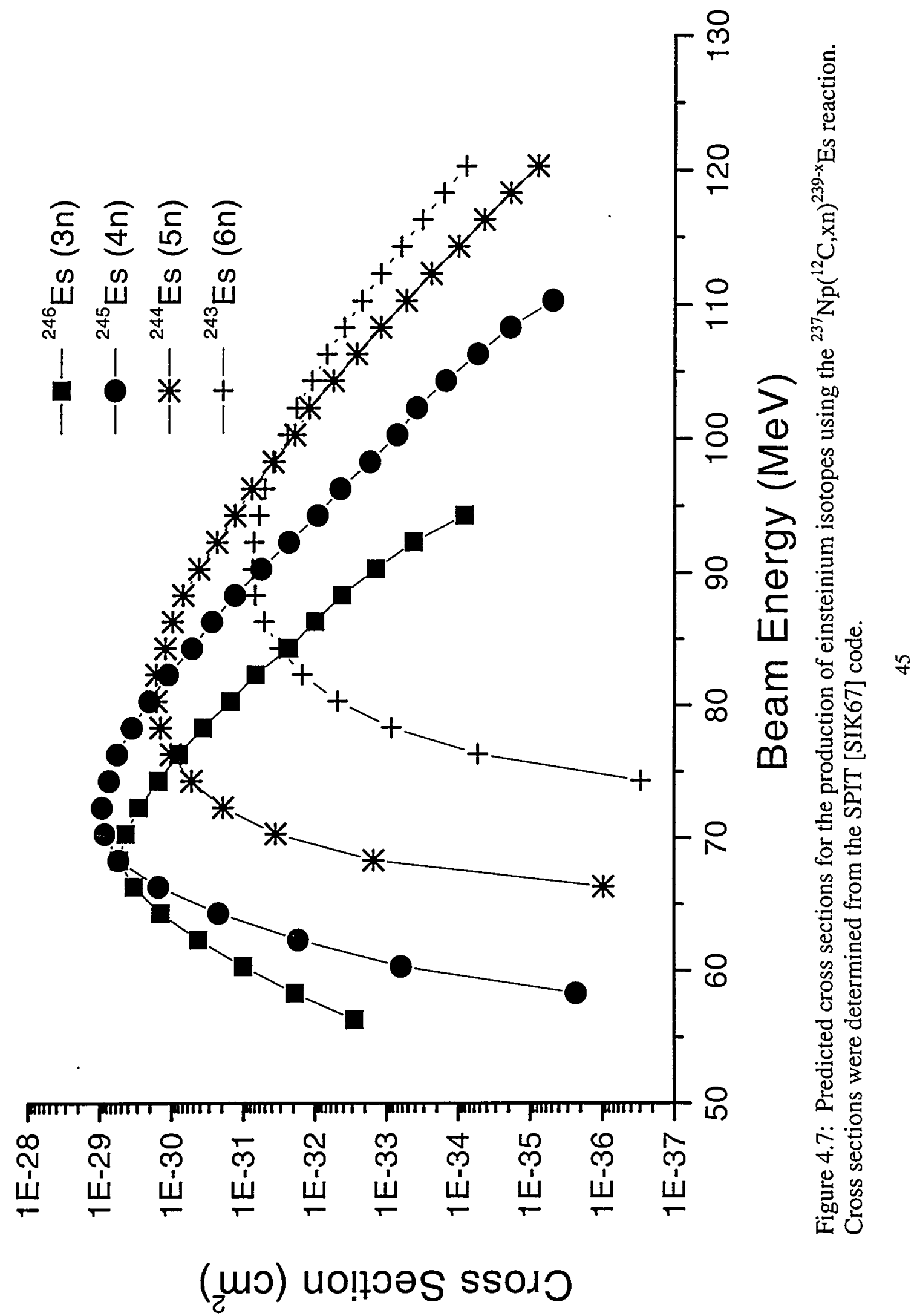




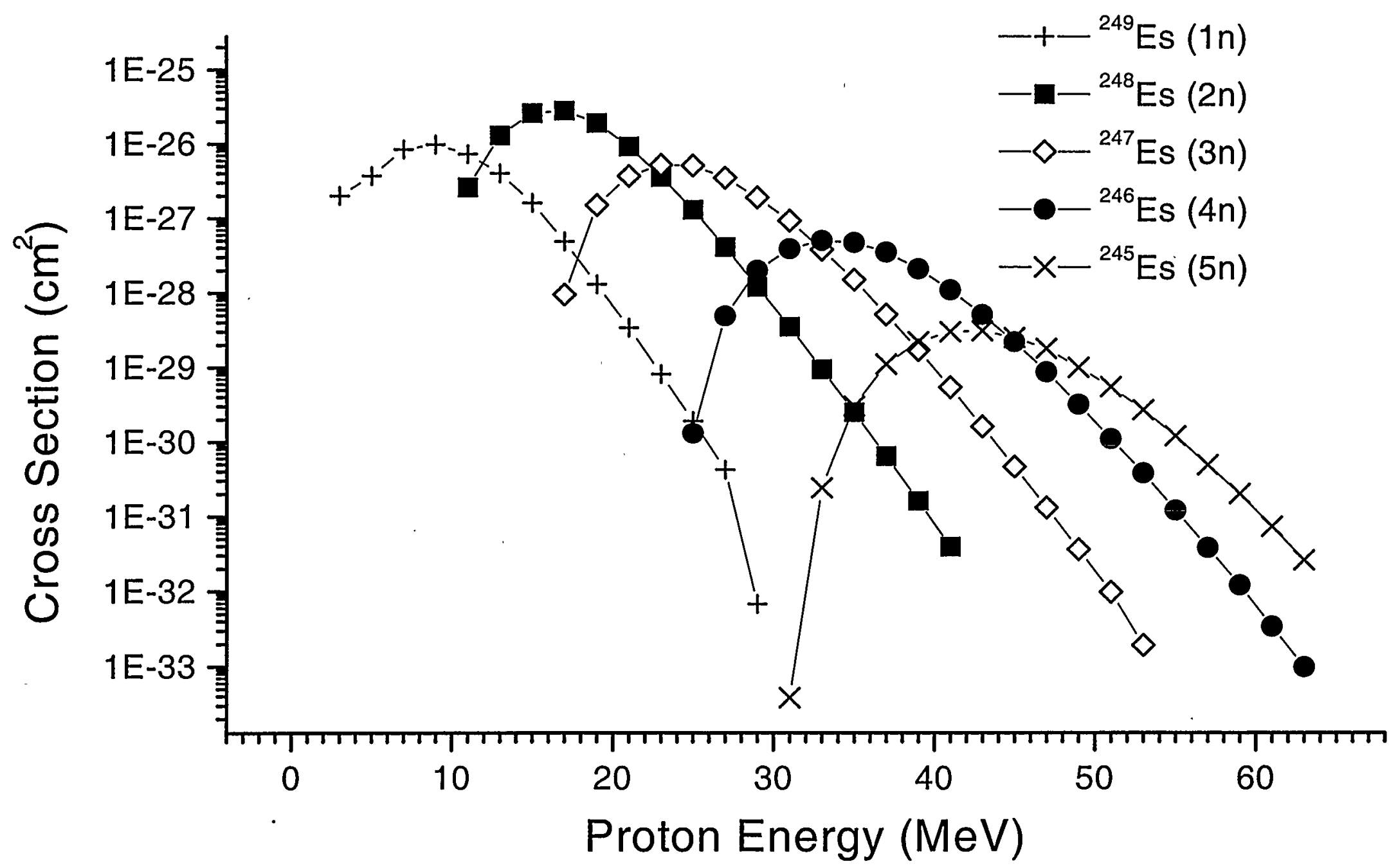

Figure 4.8: Predicted cross sections for the production of einsteinium isotopes using the ${ }^{249} \mathrm{Cf}(\mathrm{p}, \mathrm{xn})^{250-\mathrm{x}} \mathrm{Es}$ reaction. Cross sections were determined using the SPIT [SIK67] code. 


\section{Experimental Procedures}

\subsection{On-line Procedures}

On-line measurements of $\alpha$-particles and fission fragments were made using the "Merry-Go-Around" (MG) rotating wheel system [HOFFM90]. The MG consists of a $51-\mathrm{cm}$ diameter wheel with 80 thin polypropylene foils $\left(40 \pm 10 \mu \mathrm{g} / \mathrm{cm}^{2}\right.$ thick) positioned around the periphery. Once reaction products are swept out of the target chamber via the $\mathrm{He} / \mathrm{KCl}$ gas-jet system described in Chapter 4, the $\mathrm{KCl}$ aerosol plus activity is deposited on one of these thin foils. The wheel is then stepped at preset time intervals between a series of six detector stations. Each station consists of a pair of passivated ion-implanted silicon (PIPS) semiconductor detectors positioned directly above and below the wheel. After a sample is collected, it passes through each detector station until it leaves station number six where it is no longer counted. This system offers the benefit of a continuous collection and counting apparatus. While one sample is being collected, six others are being counted. After one or more complete revolutions of the wheel (depending on the stepping time) the wheel is replaced with a clean one to prevent the buildup of $\mathrm{KCl}$ on the foils, which worsens the $\alpha$-energy resolution during experiments. It also prevents the buildup of long-lived fission activities on the foils. Figure 5.1 shows the MG from a top view, and Figure 5.2 shows a cross-sectional view of the detector stations.

The MG apparatus was used in ECDF experiments to look for coincident pairs of fission fragments coming from the delayed fission process. The MG was also used to measure $\alpha$-particles in order to determine how much of a particular isotope was produced 


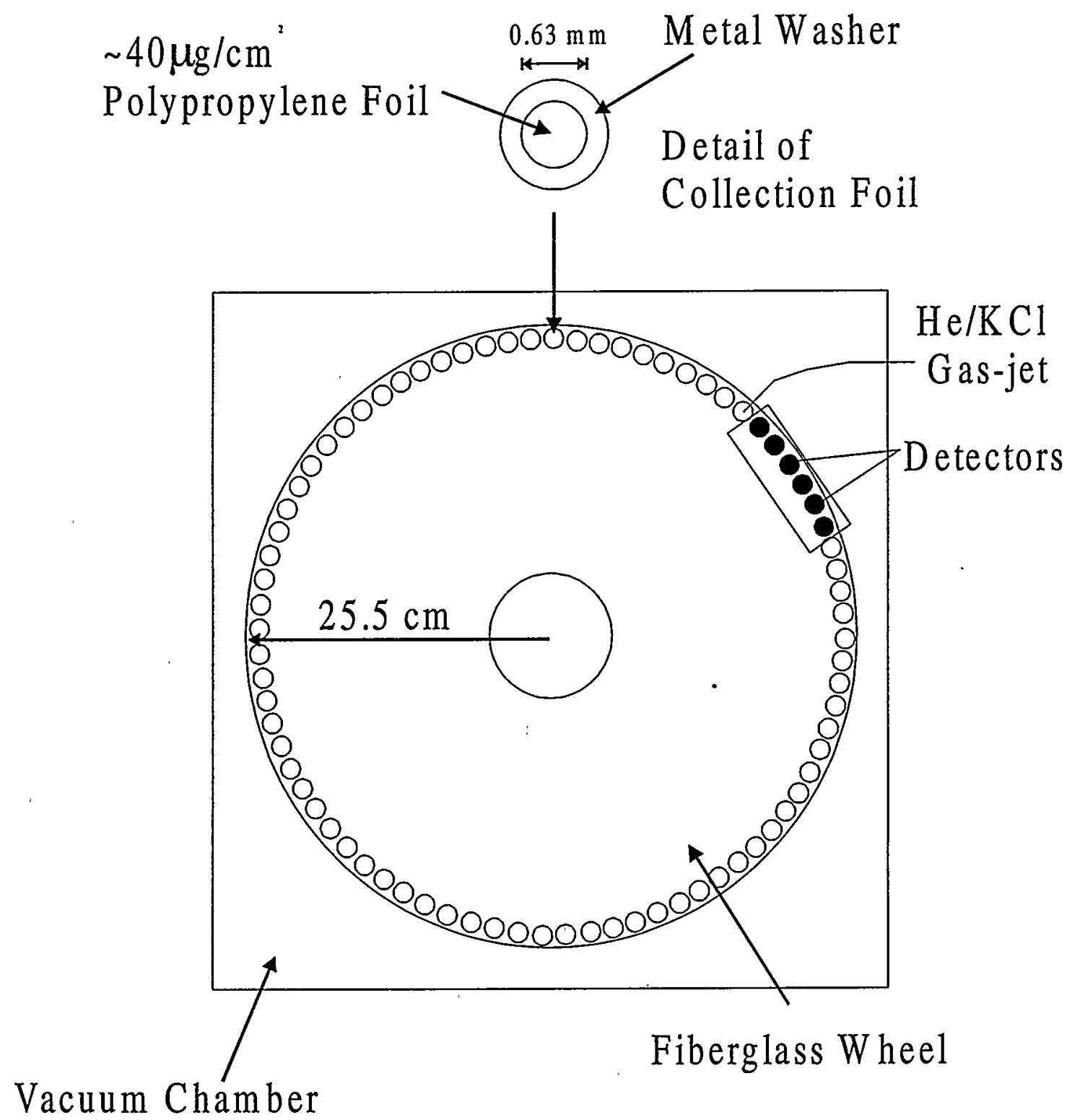

Figure 5.1: Top view of the MG rotating wheel detection system. There are 80 positions around the periphery of the wheel, which rotates in a clockwise direction. 

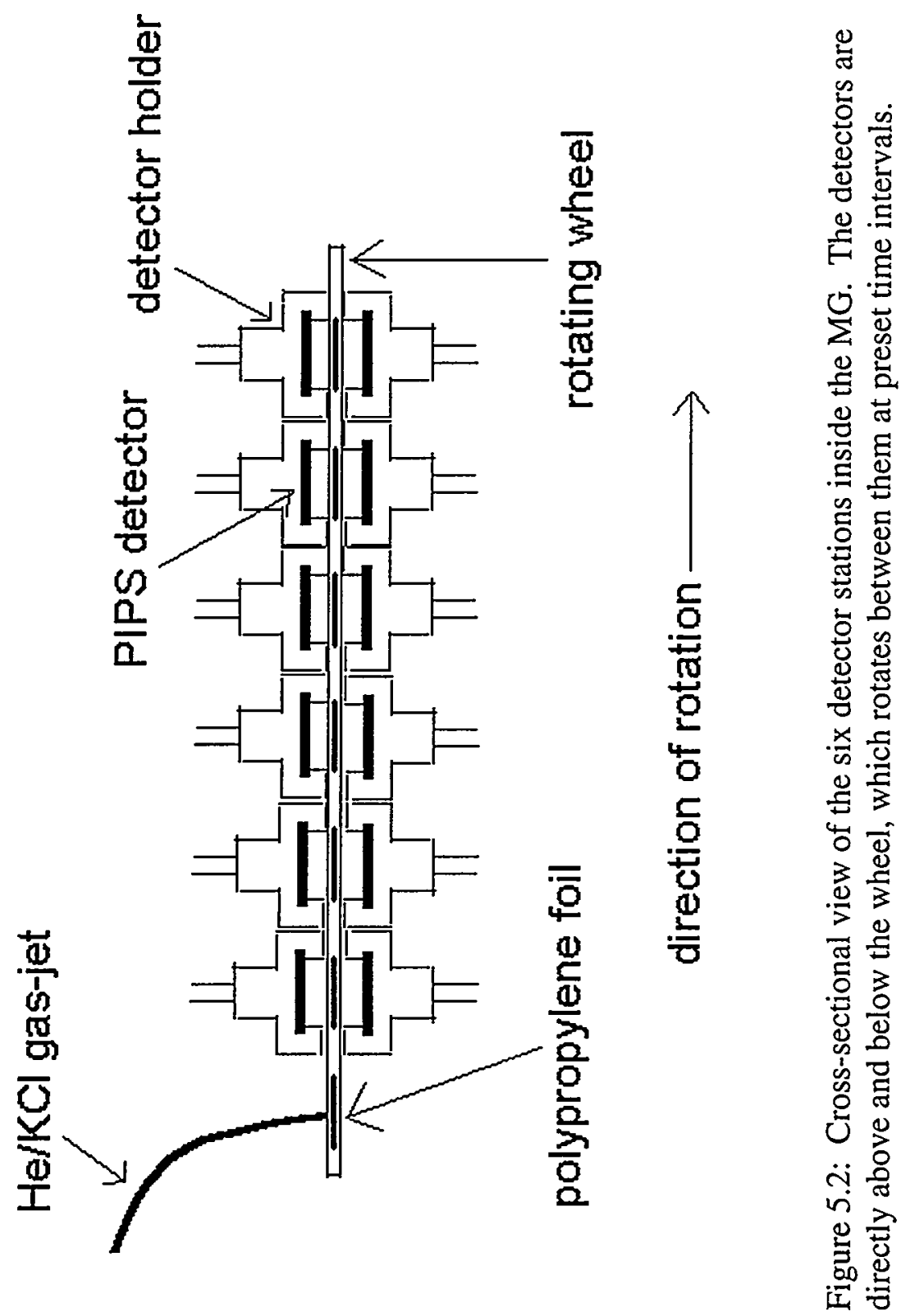
during a cyclotron bombardment. This was especially important in the study of nuclides with half-lives too short for chemical separations prior to counting. In some cases, chemical separations were used in addition to the $\mathrm{MG}$ measurements for a more complete study of a particular nuclide. More details of these experiments will be presented in later sections.

Data from the detectors was recorded in event-by-event mode using the CHAOS [RAT91] data acquisition software. Each $\alpha$-particle or fission fragment detected was tagged with the detector number, the channel it appeared in (proportional to its energy) and the time that it occurred. Data analysis was done following each MG experiment using both the CHAOS program itself and analysis codes written in the FORTRAN programming language that sorted the data and made appropriate histograms. A basic program [GRE96] was first used to sort the raw data, and then a subroutine appropriate for the parameters of each particular experiment was added in order to display and analyze various spectra. This allowed for greater flexibility in designing and running experiments using the MG. The MLDS code [GRE91] was subsequently used for the determination of half-lives and activities. This program fits multi-component decay curves using a combination of maximum likelihood techniques based on Poisson statistics and iterative curve fitting using the simplex method. The results are estimates of half-lives for each component, as well as estimates of the initial activities of the nuclides at a time zero defined by the user. This program is especially useful when the counting statistics are poor, as in the case of low production rates.

The PIPS detectors used in the MG had an $\alpha$-particle efficiency of $32 \%$ and the efficiency for detecting a coincident pair of fission fragments in the top and bottom 
detectors was approximately $60 \%$. The $\alpha$-resolution in the top detectors was $40-\mathrm{keV}$ fullwidth-at-half maximum (FWHM) and the resolution of the bottom detectors was 100keV FWHM due to energy degradation of the particles as they passed through the polypropylene foil. The detectors were calibrated before each experiment so that channel numbers could later be converted into energies. $\alpha$-energies were determined using a ${ }^{212} \mathrm{~Pb}$ calibration source on $\mathrm{Al}$ foil. This source provides two $\alpha$-peaks with energies of $6.062 \mathrm{MeV}$ and $8.784 \mathrm{MeV}$. Figure 5.3 shows a typical $\alpha$-calibration spectrum. The gains on the amplifiers connected to the detectors were adjusted so that the each channel was equivalent to approximately $6 \mathrm{keV}$. Fission energies were determined by calibrating the system with a ${ }^{252} \mathrm{Cf}$ source evaporated on a thin polypropylene foil. Figure 5.4 shows a typical fission calibration spectrum. Fission fragment energies were determined from these calibrations using the method of Schmitt, Kiker and Williams [SCH65] and the constants of Weissenberger et al. [WEI86]. $78.4 \mathrm{MeV}$ and 102.6 MeV were used for the most probable low and high peak energies from the spontaneous fission of ${ }^{252} \mathrm{Cf}$, respectively.

The MG was set with different stepping times depending on the type of experiment being performed and the particular nuclide under study. Experiments investigating ECDF in ${ }^{242}$ Es used both $10-\mathrm{s}$ and 20 -s steps. In each case the wheel was allowed to collect and count samples for 1-h before it was replaced with a clean wheel. This corresponded to four and a half wheel revolutions of 10-s steps and two and a quarter revolutions with 20-s steps. Hingmann et al. [HIN85] had estimated the half-life of ${ }^{242} \mathrm{Es}$ to be between 5 and $25 \mathrm{~s}$. The stepping times of $10 \mathrm{~s}$ and $20 \mathrm{~s}$ were chosen since the half-life was thought to be in this range and it would be possible to monitor the decay 


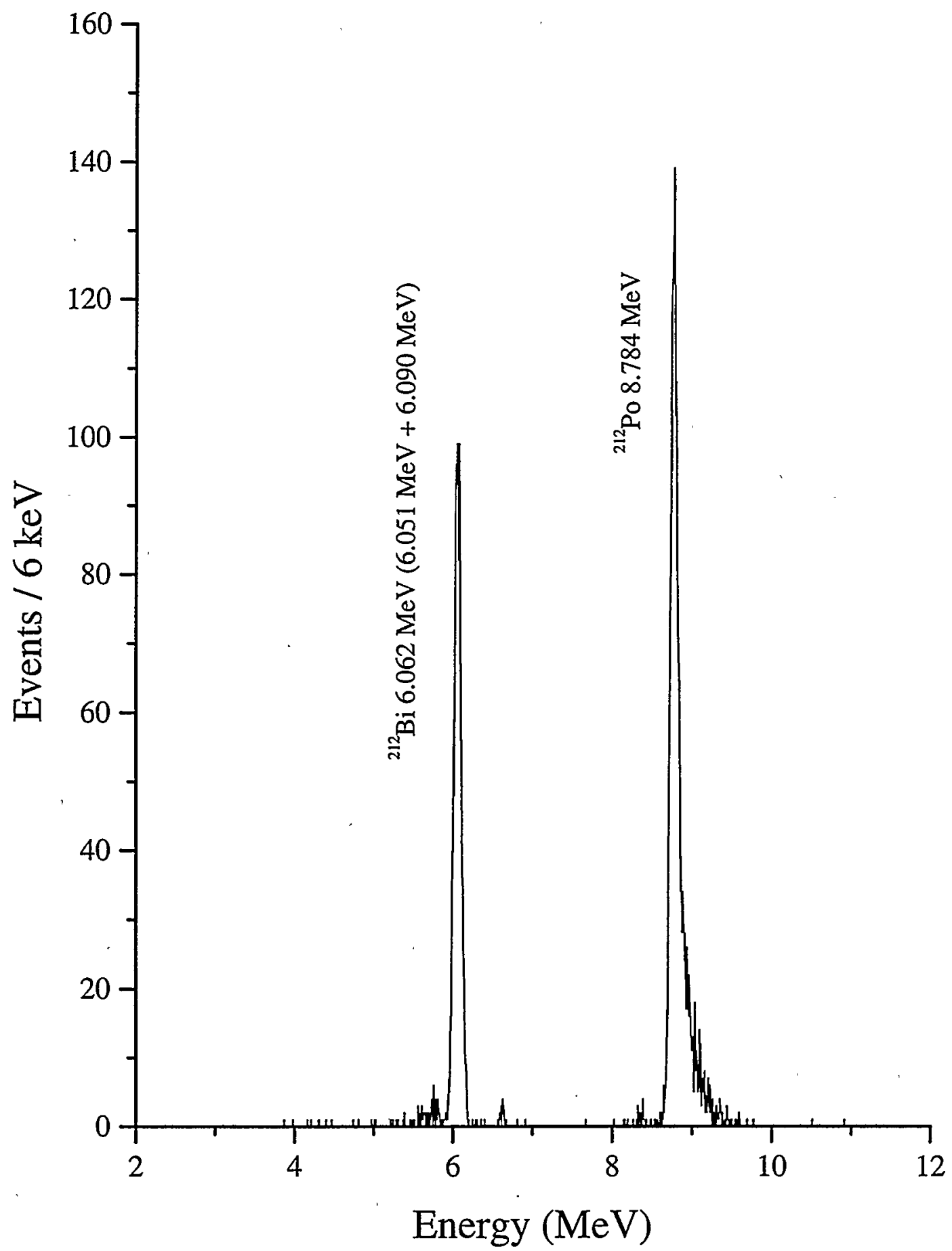

Figure 5.3: $M G \alpha$-calibration spectrum using a ${ }^{212} \mathrm{~Pb}$ source. The high-energy tail on the ${ }^{212} \mathrm{Po}$ peak is due to summing of the ${ }^{212} \mathrm{Bi}$ beta with the ${ }^{212}$ Po $\alpha$-particle. 


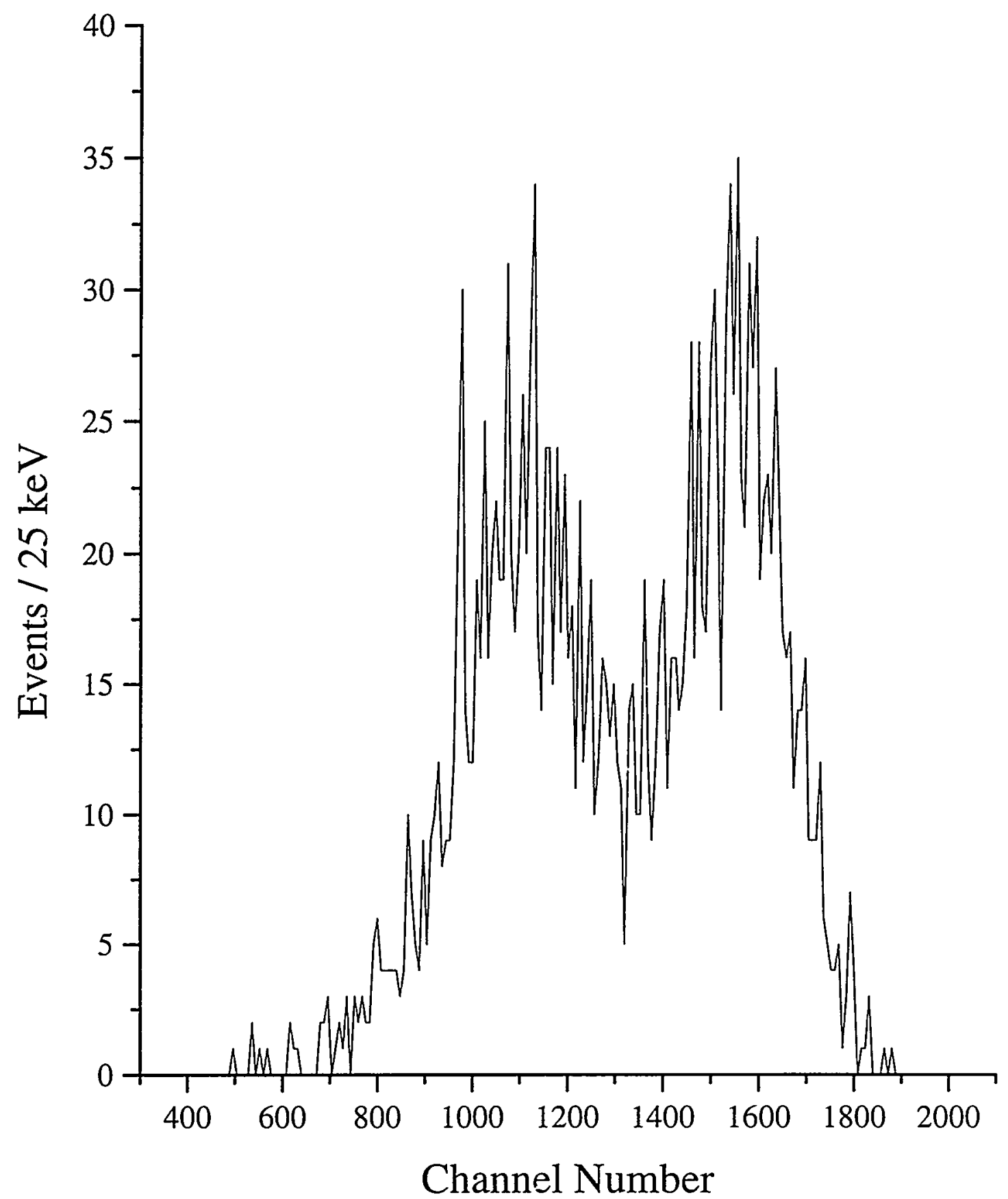

Figure 5.4: $\mathrm{MG}$ fission calibration spectrum using a ${ }^{252} \mathrm{Cf}$ source on thin polypropylene foil. 
of ${ }^{242}$ Es over three to six half-lives. In the study of ECDF in ${ }^{244} \mathrm{Es}$, the initial experiment used a 10-s stepping time for $1 \mathrm{~h}$. The half-life of ${ }^{244} \mathrm{Es}$ was known to be $37 \mathrm{~s}$ [FIR96] so this time was probably too short, covering only two half-lives. Subsequent ${ }^{244}$ Es ECDF experiments used à more appropriate stepping time of $30 \mathrm{~s}$ for $80 \mathrm{~min}$, corresponding to two complete revolutions of the wheel. ${ }^{246}$ Es and ${ }^{248}$ Es are longer lived (7.7 min and 26 min, respectively [FIR96]) so longer stepping times had to be used in order to follow the decay of these nuclides during their ECDF experiments. For ${ }^{246} \mathrm{Es}$, a 1-min stepping time was run for $80 \mathrm{~min}$ (one complete revolution) and a 2-min time interval was used for ${ }^{248} \mathrm{Es}$ for $1 \mathrm{~h}, 20 \mathrm{~min}$ (one complete revolution). The time the samples spent between the six detector pairs only corresponded to one half of a half-life of ${ }^{248} \mathrm{Es}$, but a longer stepping time would have resulted in a larger deposit of $\mathrm{KCl}$ on the foils, resulting in worse $\alpha$-resolution in the detectors. Also, a larger salt deposit is more dangerous because it could flake off the foil easier than a smaller deposit, presenting a contamination hazard. The best way to study a longer-lived nuclide like ${ }^{248} \mathrm{Es}$ is to perform a chemical separation on the sample, followed by a much longer counting interval. This was also done and will be described in later sections of this chapter.

\subsection{Chemical Separations and X-ray/Fission Correlation}

\section{Studies}

While the MG system is ideal for studying the decay properties of shorter-lived isotopes, it has a disadvantage in that there are often large amounts of background activities in the samples. These are produced via the interaction between the beam and 
impurities in the target and can overwhelm a much smaller peak in the spectrum. For the study of longer-lived nuclides, it is therefore desirable to remove reaction products from the collection site and perform chemical separations on them to remove interfering activities and improve the quality of the resulting spectra. It is also possible with longerlived nuclides to look for coincidences between $\mathrm{x}$-rays coming from the initial EC-decay and fission fragments. This method was first used by Hall et al. [HAL89B] to prove the existence of the ECDF decay mode. The number of $\mathrm{x}$-rays detected is a direct measure of the total number of EC-decays that has occurred. By measuring fission fragments in coincidence with $\mathrm{x}$-rays, background sources of fission fragments from other elements are eliminated. This method also confirms the identity of the EC-parent and proves that any fission events measured indeed come from a delayed fission event.

This method was used to try and study ECDF of ${ }^{246}$ Es and ${ }^{248}$ Es. After bombardment, the activity was collected and measured first using the MG as described in section 5.1. This was done to measure $\alpha$-energies and the mass-yield and total kinetic energy (TKE) distributions of the fission fragments. After one complete revolution of the MG wheel, a chemical extraction was done on some of the most recently collected samples to remove $\mathrm{KCl}$ and other impurities before the $\mathrm{x}$-ray/fission coincidence measurement. Thenoyltrifluoroacetone (TTA) is known to complex $3+$ and higher oxidation state ions from aqueous solutions of $\mathrm{pH} 4$ to 5 [POS61]. The $\mathrm{KCl}$ deposits from five to seven polypropylene foils were dissolved in $25 \mu \mathrm{L}$ of a pH 5 buffer solution of acetic acid and sodium acetate. $\mathrm{Es}^{3+}$ and any other trivalent or higher species were extracted into an equal volume of $0.5 \mathrm{M}$ TTA in toluene. The organic phase was removed and evaporated on a glass cover slip for analysis. To get an estimate of the 
chemical yield for this process, one of the polypropylene foils from the MG wheel was placed on a silicon surface barrier detector operated under vacuum. The yield was determined by comparing the amount of ${ }^{249} \mathrm{Cf} \alpha$-activity on the foil to that seen after the chemical separation. Chemical efficiencies varied from $50 \%$ to approximately $90 \%$.

After a chemical separation was performed, the glass cover slip with the dried sample was placed in the $\mathrm{x}$-ray/fission coincidence setup. This consisted of a silicon surface barrier detector operated in air sandwiched between two germanium x-ray detectors. A schematic of this arrangement is shown in Figure 5.5. Fission fragments and $\alpha$-particles are detected in the Si detector while $\mathrm{x}$-rays are recorded with the $\mathrm{Ge}$ detectors. The CHAOS acquisition system was used to record signals from all three detectors.

Both the yield check detector and the detector in the $\mathrm{x}$-ray/fission setup were calibrated with a ${ }^{252} \mathrm{Cf}$ source evaporated on $\mathrm{Pt}$ foil. The yield check detector was set so there were approximately $10 \mathrm{keV}$ per channel and the coincidence detector was approximately $6 \mathrm{keV}$ per channel. Both detectors had an efficiency of approximately $30 \%$. The $\mathrm{x}$-ray detectors were calibrated with a ${ }^{249} \mathrm{Cf}$ source on $\mathrm{Ta}$ foil. They were both adjusted so that each channel corresponded to $1 \mathrm{keV}$. The efficiency of both detectors was determined by measuring $\alpha$-x-ray coincidences from ${ }^{249} \mathrm{Cf}$, and was approximately $10 \%$ for both detectors. The resolution of the $\mathrm{x}$-ray detectors was $1.75-\mathrm{keV}$ FWHM for the low-energy $\mathrm{x}$-ray region (below $60 \mathrm{keV}$ ) and 3-keV FWHM for higher energy $\mathrm{x}$-rays and $\gamma$-rays. The $\alpha$-resolution of the coincidence detector was approximately $125-\mathrm{keV}$ FWHM. This poor resolution was due to the fact that this detector was operated in air. The resolution of the yield check detector was comparable to the detectors in the MG 
system. A sample $\mathrm{x}$-ray calibration is shown in Figure 5.6 with the prominent $\mathrm{x}$-rays from the ${ }^{249} \mathrm{Cf}$ source identified.

\subsection{Excitation Function Procedures}

\subsection{1 ${ }^{14,15} \mathrm{~N}+{ }^{233} \mathrm{U}$ Excitation Functions}

As discussed in Chapter 4, excitation functions for the ${ }^{233} U\left({ }^{14} \mathrm{~N}, \mathrm{xn}\right){ }^{247-\mathrm{x}} \mathrm{Es}$ and ${ }^{233} U\left({ }^{15} \mathrm{~N}, \mathrm{xn}\right){ }^{248-\mathrm{x}}$ Es reactions were measured. The beam energies were given in section 4.3. These experiments were performed with six detector pairs in the MG. Data were recorded using the CHAOS acquisition system as described in section 5.1 and FORTRAN programs were used for subsequent sorting and histogramming of the data as described earlier.

The following procedure was used for both ${ }^{14} \mathrm{~N}$ and ${ }^{15} \mathrm{~N}$ beams. At each energy, four different stepping times were used in the MG. First a 5-s stepping time was run for 20 min, corresponding to three revolutions of the wheel. This was followed by a 10-s stepping time for $30 \mathrm{~min}$, resulting in two and a quarter wheel revolutions. The wheel was replaced with clean foils in between each stepping time. Then 1-min and 2-min stepping times were each run for one complete wheel revolution $(80 \mathrm{~min}$ and $160 \mathrm{~min}$, respectively.) After the 2-min wheel was finished, the last six samples that were collected were counted while the wheel was stationary during the time that the cyclotron staff used to tune the next beam energy. This allowed us to look for longer-lived activities after most of the shorter interfering activities had already decayed. This procedure was followed at each energy for both ${ }^{14} \mathrm{~N}$ and ${ }^{15} \mathrm{~N}$ projectiles. 


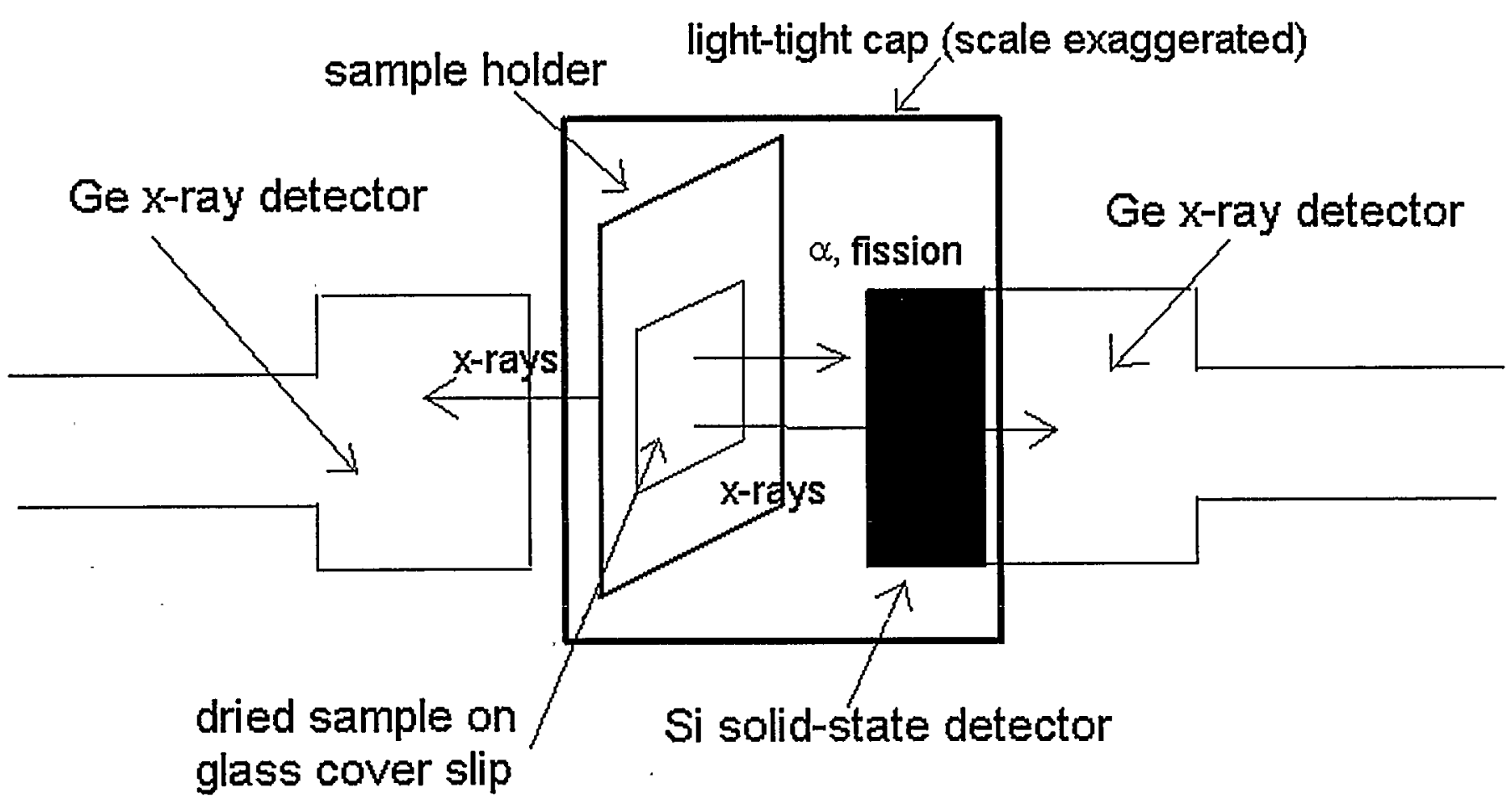

Figure 5.5: $\mathrm{X}$-ray/fission coincidence measurement setup. The glass cover slip with the sample is inserted into a sample holder, which slips between the two Ge detectors. The Si detector is contained within a light-tight cap placed on the right-hand Ge detector. 


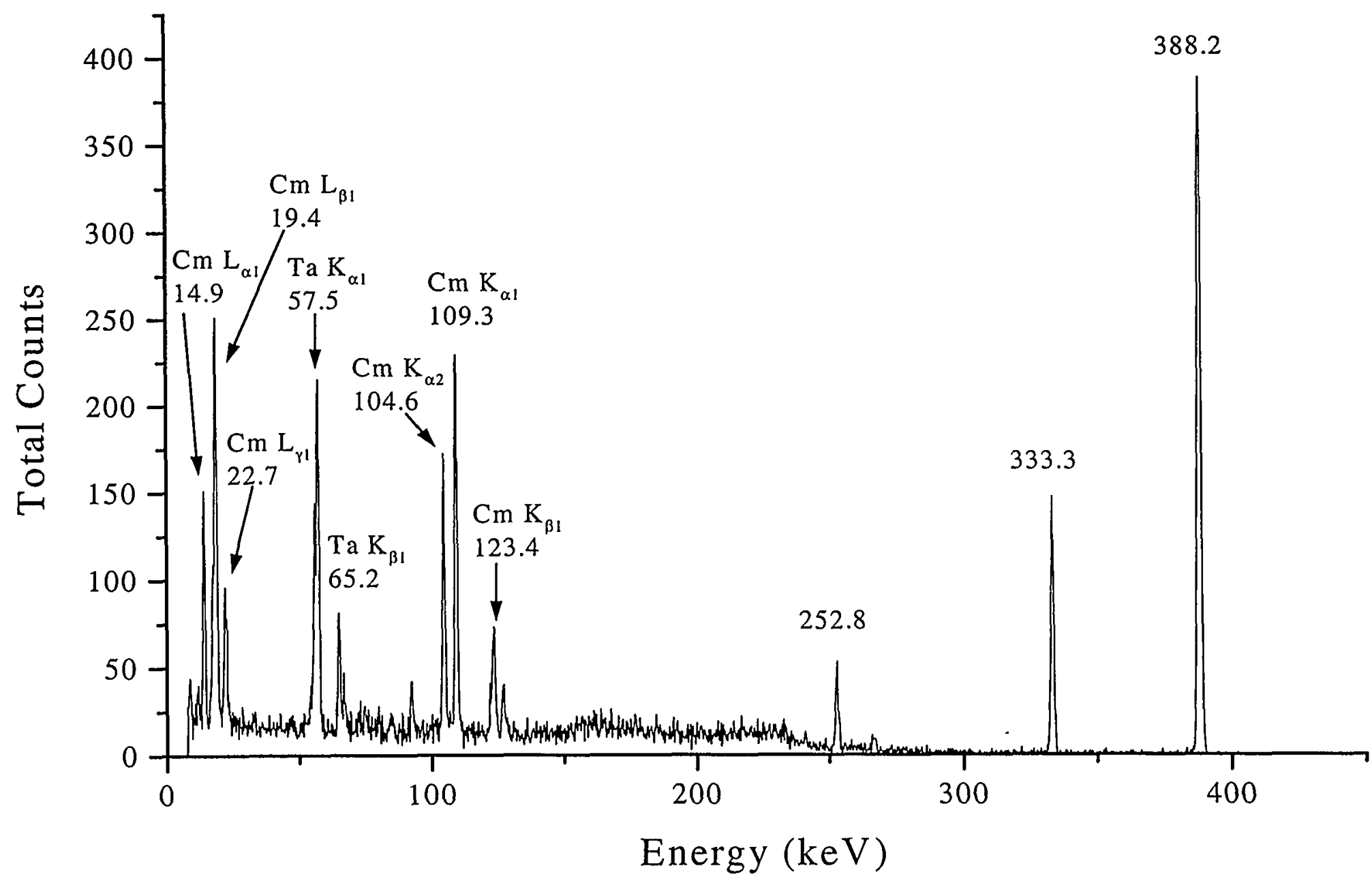

Figure 5.6: X-ray calibration using a ${ }^{249} \mathrm{Cf}$ source deposited on Ta foil. The ${ }^{249} \mathrm{Cf}$ undergoes $\alpha$-decay to ${ }^{245} \mathrm{Cm}$, which produces the $\mathrm{x}$-rays shown in the spectrum. The higher energy peaks are $\gamma$-ray transitions from excited states in ${ }^{245} \mathrm{Cm}$. $\mathrm{X}$-rays from the fluorescence of the Ta foil are also shown in the spectrum. 


\subsection{2 $\mathrm{p}+{ }^{249} \mathrm{Cf}$ Excitation Function}

To investigate the production of heavier einsteinium isotopes $\left({ }^{245} \mathrm{Es}\right.$ to $\left.{ }^{249} \mathrm{Es}\right)$, an excitation function of the ${ }^{249} \mathrm{Cf}(\mathrm{p}, \mathrm{xn})^{250-\mathrm{x}} \mathrm{Es}$ reaction was measured at four energies (see section 4.3.) These nuclides have half-lives between $1.1 \mathrm{~min}$ and $1.7 \mathrm{~h}$., so a combination of MG counting and chemical analysis was used. The following procedure was used at each proton energy. To study the shorter-lived nuclides, the MG was set with a 1-min stepping time and the wheel was counted for one revolution $(80 \mathrm{~min})$. The CHAOS program was used to record data. After collection and measurement was finished, one of the foils with a $\mathrm{KCl}$ deposit was removed and placed in a yield check detector as described in section 5.2. Six of the most recently collected $\mathrm{KCl}$ deposits were dissolved in $25 \mu \mathrm{L}$ of a pH 5 buffer solution of sodium acetate and acetic acid. A TTA extraction (see section 5.2) was performed and the organic phase containing einsteinium and any other $3+$ ions was dried on a glass cover slip. This sample was counted on another silicon-surface barrier detector, just like the one used for counting yield check samples. These data were also recorded using the CHAOS acquisition system.

After the chemical separation was complete, a new wheel was placed in the MG and a stepping time of $2 \mathrm{~min}$ was used. After one complete revolution of the wheel (160 min), one foil was removed and counted as a yield check. A TTA extraction was done on the six most recently collected samples as described above and was subsequently counted. The chemistry samples were counted while the cyclotron staff tuned the next beam energy. This entire process was repeated for all four proton energies used. FORTRAN programs were later used to sort and histogram the data as described previously, both from the MG measurements and the chemical samples. 
In conjunction with the ${ }^{249} \mathrm{Cf}$ plus proton excitation function experiments, catcher foils were counted to determine the gas-jet transport efficiency of the LIM target system. A $1.2-\mathrm{mg} / \mathrm{cm}^{2}$ piece of gold foil was mounted on a ring and placed after one of the thin ${ }^{249} \mathrm{Cf}$ LIM targets so it would collect reaction products as they exited the target. The target was $14.6-\mu \mathrm{g} / \mathrm{cm}^{2}$ thick and was bombarded for $1 \mathrm{~h}$. In this type of arrangement, the foil has a collection efficiency of approximately 100\% [LEY90]. The gold foil was then removed and dissolved in aqua regia. A known amount of ${ }^{241} \mathrm{Am}$ tracer was added to the activity in order to determine the overall chemical yield of the separation. The gold solution was then placed on a 3-cm high column of AG1X-8 anion exchange resin (200-400 mesh.) The $3+$ actinides were eluted from the column with concentrated $\mathrm{HCl}$ while the gold and other impurities remained on the column. The $\mathrm{HCl}$ solution containing the activity was evaporated and transferred to a Pt disk using $3 \mathrm{M} \mathrm{HCl}$. The sample was dried on the disk and then flamed to remove any residual acid. The efficiency of the chemical separation was approximately $57 \%$. The sample was counted at different time intervals using a surface-barrier detector like the ones described previously.

After the gold foil was removed, a 2.2- $\mathrm{mg} / \mathrm{cm}^{2}$ Mo foil was placed behind the same ${ }^{249} \mathrm{Cf}$ target to collect reaction products for an additional $30 \mathrm{~min}$. After bombardment, this foil was removed and counted directly without any prior chemical separation. The Mo foil was used to look for shorter-lived activities, whereas the gold foil was used to measure longer-lived activities, since the chemical procedure takes approximately $1 \mathrm{~h}$. The data from the catcher foils were collected using a standard 
acquisition system and were subsequently analyzed using original FORTRAN programs [LEE96]. 


\section{Results and Discussion}

\subsection{Electron-Capture Delayed Fission}

\section{$6.1 .1^{242} \mathrm{Es}$}

\subsubsection{Fission Properties and Half-Life}

${ }^{242}$ Es was produced via the ${ }^{233} U\left({ }^{14} \mathrm{~N}, 5 \mathrm{n}\right){ }^{242}$ Es reaction using a single target as described in Chapter 4, and reaction products were measured with the MG rotating wheel detection system described in Chapter 5 using 10-s and 20-s stepping intervals. Three separate experiments (a total of $96 \mathrm{~h}$ of beam time) were performed to study ECDF in

${ }^{242}$ Es. A total of 48 coincident fission fragment pairs were detected over the course of the three experiments. Background measurements taken before and after the experiments showed that less than one fission event could be attributed to long-lived background activities in all of the detectors. All of the fission events were ultimately summed together to determine the ${ }^{242} \mathrm{Cf}$ fission properties from the ${ }^{242} \mathrm{Es}$ precursor.

Figure 6.1 shows the decay curve of fission events from the third experiment using a 20 -s stepping interval in the MG and representing $48 \mathrm{~h}$ of beam time. The first two experiments used a 10-s stepping interval. Over half of the 48 fission events were detected during the third experiment resulting in better counting statistics than during the first two experiments. The population of states in the fissioning daughter nucleus occurs with the half-life of the EC-parent [KRE93], and the subsequent fission is instantaneous compared to this half-life. Therefore, the fission events decay with the characteristic 


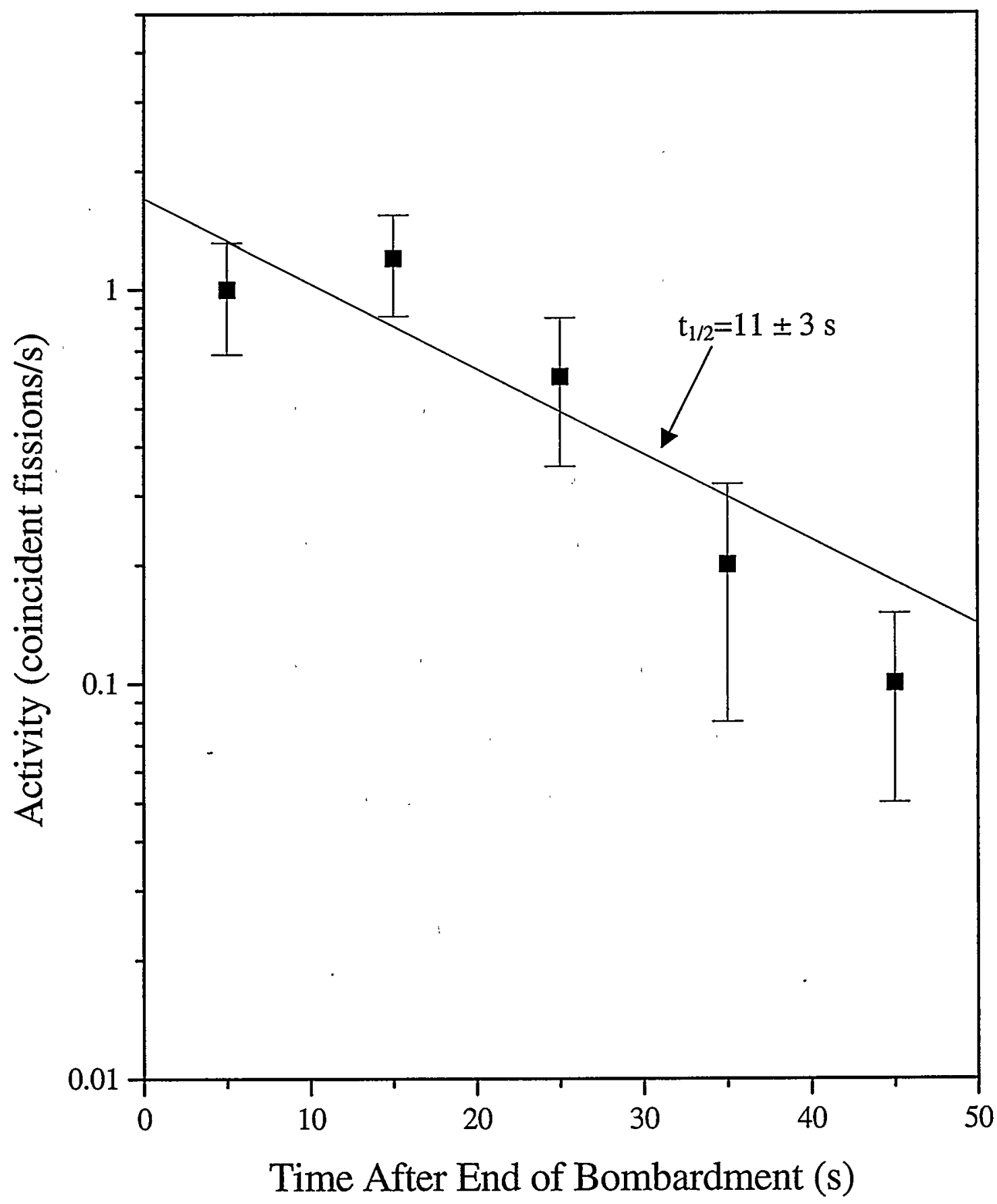

Figure 6.1: Decay curve of coincident fission events from the ECDF of ${ }^{242}$ Es. Data were taken from the third ${ }^{242}$ Es experiment using 20 -s steps in the MG. The total running time of this experiment was $48 \mathrm{~h}$. The line represents the best fit to the shorter ${ }^{242}$ Es component as determined by MLDS. 
half-life of the parent nucleus. Decay curves of coincident fission events were created from each of the three experiments, and the half-life of the events was determined using the MLDS program. There were two components evident in the decay curves generated from the data, the shorter ${ }^{242}$ Es component and a long-lived constant activity of approximately 0.1 counts/s (Figure 6.1 only shows the shorter component.) The half-life of ${ }^{242}$ Es from each of the experiments was determined to be $11 \pm 3 \mathrm{~s}$ using a twocomponent fit in MLDS. This value is consistent with the half-life of $16_{-4}^{+6}$ s reported by Ninov et al. [NIN96] but is more accurate since it is based on more events.

The half-life we measured proves that the fission events we detected were indeed from ECDF in ${ }^{242}$ Es. The only other nuclide in this region with a half-life the same order of magnitude is ${ }^{243}$ Es with $t_{1 / 2}=21 s$ [FIR96]. Based on the relationship between $Q_{E C}$ and $\mathrm{P}_{\mathrm{DF}}$ shown in Figure 2.2 and assuming a $\mathrm{Q}_{\mathrm{EC}}$ of $4.0 \mathrm{MeV}$ for ${ }^{243} \mathrm{Es}$ [MÖL95], the resulting $\mathrm{P}_{\mathrm{DF}}$ should be on the order of $10^{-5}$. This would result in a fission rate much lower than we observed during the experiments. Since there are no spontaneous fission or other delayed fission isotopes with a half-life close to $11 \mathrm{~s}$ made in the reaction of ${ }^{14} \mathrm{~N}$ with ${ }^{233} \mathrm{U}$, the fission events detected were from the ECDF process with ${ }^{242} \mathrm{Es}$ as the parent nucleus.

Fission energy calibrations were performed with a ${ }^{252} \mathrm{Cf}$ source as discussed in Chapter 5. The average neutron emission function for ${ }^{242} \mathrm{Cf}, \bar{v}(A)$, was assumed to be similar to that of ${ }^{252} \mathrm{Cf}$, normalized to an average neutron emission $\bar{v}_{t}=2.6$ (estimated from systematics in [HOFFM74].) Since the fission events in the ECDF process are preceded by EC-decays, the fission properties measured during the experiments were for ${ }^{242} \mathrm{Cf}$, the EC-daughter. Figure 6.2 shows the highly asymmetric mass-yield distribution 


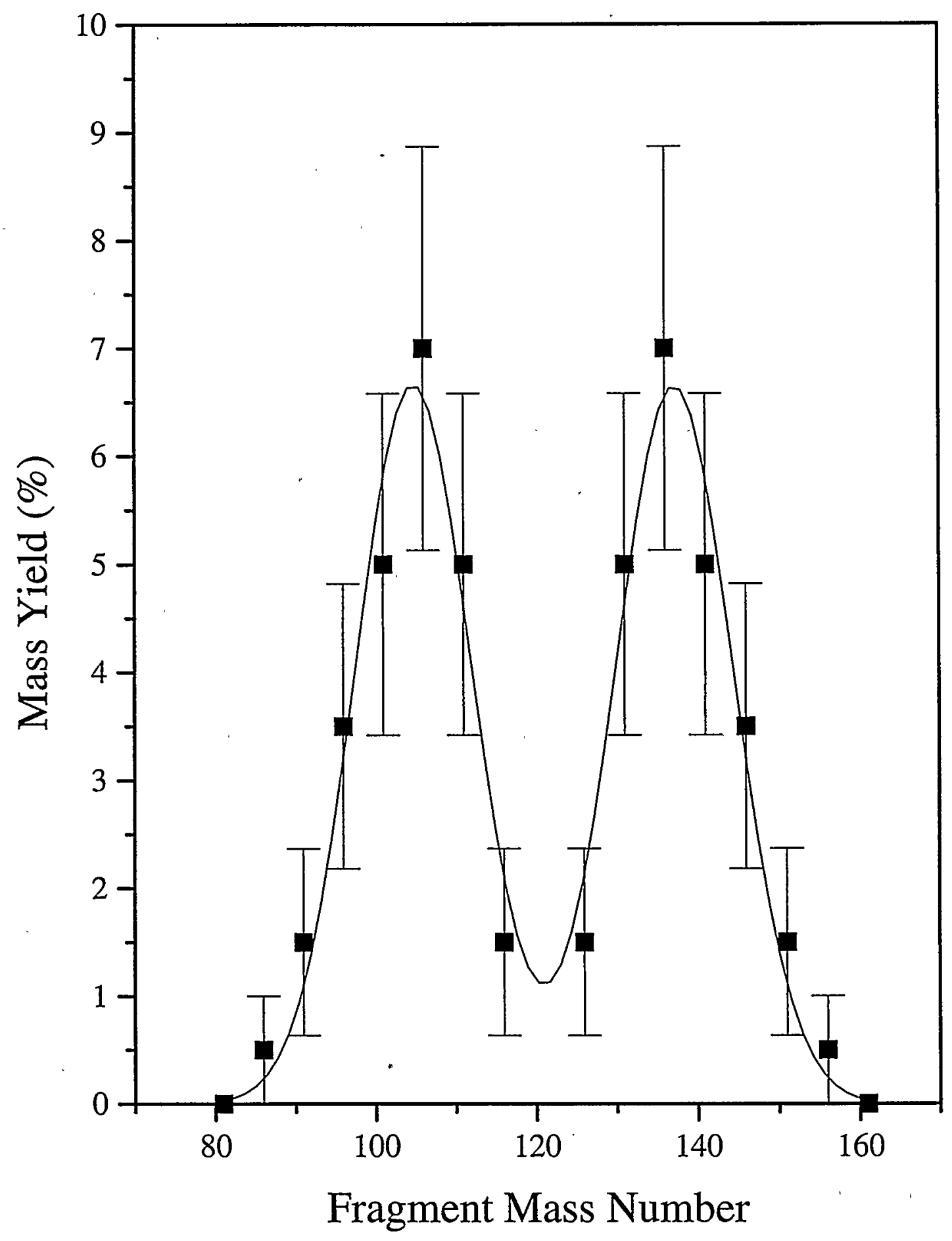

Figure 6.2: Pre-neutron emission mass-yield distribution for the ECDF of ${ }^{242} \mathrm{Es}$. The fissioning species is ${ }^{242} \mathrm{Cf}$. The data were averaged over five mass units. 
obtained for ${ }^{242} \mathrm{Cf}$. The distribution is clearly double-humped and has a well-defined valley. There is no evidence of a symmetric component.

The pre-neutron emission total kinetic energy (TKE) distribution for coincident fission fragments from ${ }^{242} \mathrm{Cf}$ is shown in Figure 6.3. The average pre-neutron TKE was $183 \pm 18 \mathrm{MeV}$. The errors quoted for TKE values represent one standard deviation. Table 6.1 gives a summary of kinetic energy and mass properties for ${ }^{242} \mathrm{Cf}$. Since the ${ }^{252} \mathrm{Cf}$ calibration source was on the same polypropylene foil used on the MG wheel during experiments, no correction was needed to account for energy degradation of fission fragments through the foil en route to the bottom detectors. Also, no correction was needed to account for the approximately $10 \mu \mathrm{g} / \mathrm{cm}^{2}$ of $\mathrm{KCl}$ [KRE94A] deposited on each foil by the gas-jet transport system because the amount of energy lost by fission Table 6.1. Properties of the measured post-neutron-emission (Post-n) and calculated initial pre-neutron-emission (Pre-n) fragment kinetic energy and mass distributions for ${ }^{242} \mathrm{Es} \mathrm{ECDF}$ and the ${ }^{252} \mathrm{Cf}$ standard measured in the same system. Once the ${ }^{252} \mathrm{Cf}$ preneutron-emission fragment energies are calculated, these values are used for calibration input parameters. Energies are given in $\mathrm{MeV}$, based on the SKW [SCH65] calibration method with the Weissenberger constants [WEI86].

\begin{tabular}{|lcccc|}
\hline & \multicolumn{2}{c}{${ }^{242}$ Cf ECDF } & \multicolumn{2}{c|}{${ }^{252}$ Cf Standard } \\
& Pre-n & Post- $n$ & Pre- $n$ & Post-n \\
\hline Average TKE & 183 & 182 & 180 & 177 \\
$\sigma$ & 18 & 20 & 15 & 12 \\
Most probable TKE & 182 & 181 & 181 & 178 \\
$\sigma$ & 20 & 18 & 14 & 13 \\
FWHM $^{\mathrm{a}}$ & 47.0 & 42.3 & 32.9 & 30.6 \\
Light fragment energy $^{\mathrm{b}}$ & 78.3 & 76.8 & 77.2 & 76.3 \\
$\sigma$ & 9.6 & 10.3 & 9.7 & 9.8 \\
Heavy fragment energy $^{\mathrm{b}}$ & 105 & 102 & 103 & 101 \\
$\sigma$ & 12 & 12 & 8 & 8 \\
Light fragment mass $^{\mathrm{c}}$ & 104 & N/A & 108 & N/A \\
Heavy fragment mass $^{\mathrm{c}}$ & 138 & N/A & 144 & N/A \\
\hline
\end{tabular}

${ }^{a}$ Full width at half maximum, calculated from $2.35 \sigma$ for Gaussian fit to the top half of the peak. ${ }^{\mathrm{b}}$ These represent most probable values.

${ }^{\mathrm{c}}$ Masses calculated from most probable pre-neutron energies. 


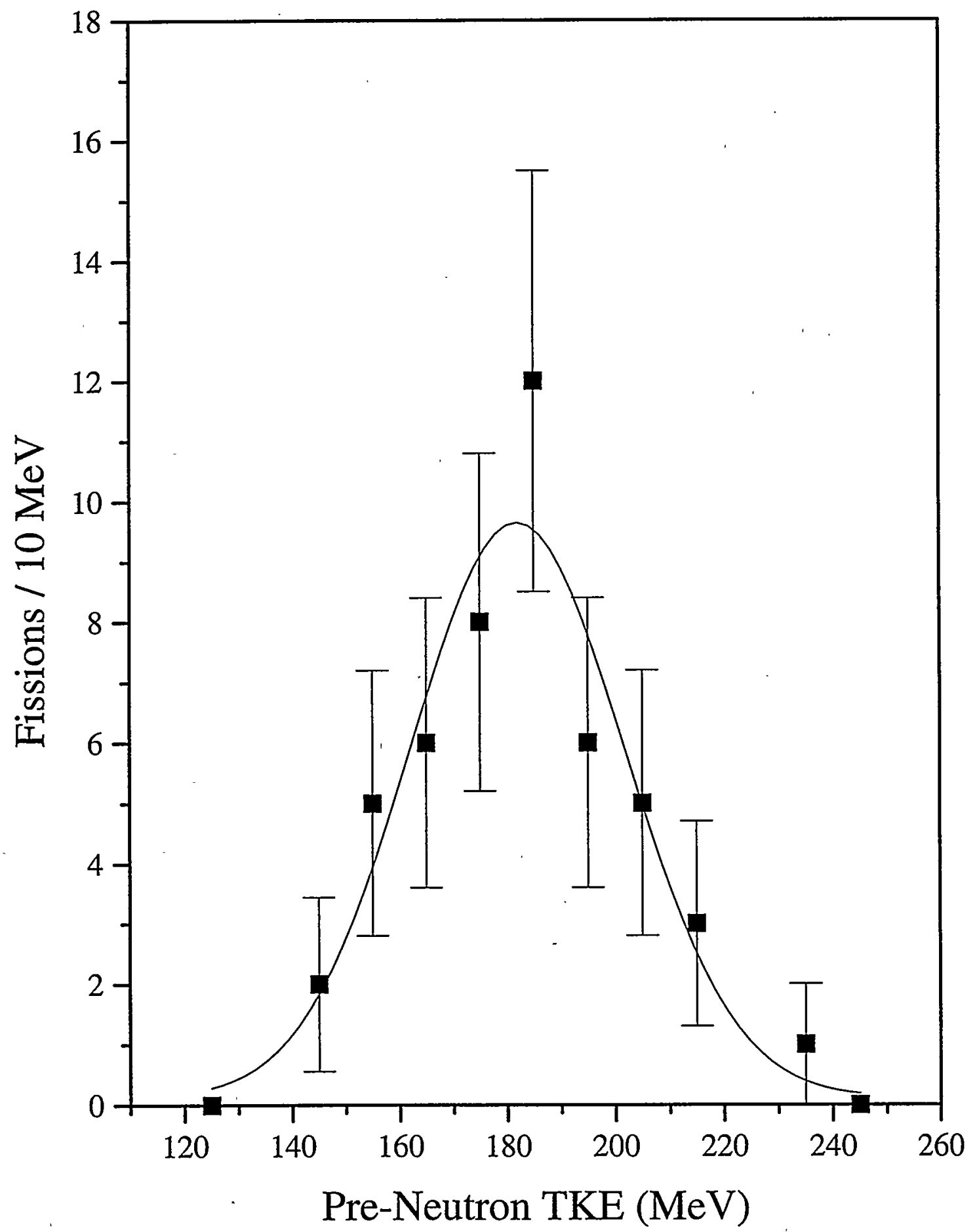

Figure 6.3: Pre-neutron emission total kinetic energy distribution (TKE) for the ECDF of ${ }^{242} \mathrm{Es}$. The fissioning species is ${ }^{242} \mathrm{Cf}$. The line is a Gaussian fit to the data. The data are in groupings of $10 \mathrm{MeV}$. 
fragments as they traveled through the $\mathrm{KCl}$ was only $0.2-0.4 \mathrm{MeV}$ [FIR96]. The average TKE value of $183 \mathrm{MeV}$ agrees within error with the empirical predictions of $191 \mathrm{MeV}$ and $194 \mathrm{MeV}$ of Viola et al. [VIO85] and Unik et al. [UNI73], respectively.

The mass-yield distribution of fission fragments is strongly influenced by shell effects [HOFFM89]. The mass distributions of fragments from ${ }^{235} \mathrm{U}$ to ${ }^{255} \mathrm{Fm}$ are all highly asymmetric with the heavy fragment having a nearly spherical, closed shell configuration due to the $\mathrm{N}=82$ spherical neutron shell and possibly the $\mathrm{Z}=50$ spherical proton shell. In the region of ${ }^{257} \mathrm{Fm}$ the mass splits become highly symmetric with both fragments having nearly spherical closed shell configurations arising from the $Z=50$ spherical proton shell and proximity to the $\mathrm{N}=82$ neutron shell. In the radium region there has been evidence of a three-humped mass-distribution [ITK88], proving that both a symmetric and asymmetric mode can simultaneously be involved in the fission process [JEN58]. The mass-yield distributions become more broadly symmetric with increasing excitation energy of the system [KON69]. As the excitation energy increases, the shell effects tend to be washed out, causing the fission to proceed more as a liquid drop model process [VAN73]. The ECDF process can impart excitation energy up to the QEC value to the fissioning system [KRE93], meaning that up to $5 \mathrm{MeV}$ of excitation energy can be imparted to the fissioning nucleus in some systems. It is possible that a symmetric mode may exist in addition to the asymmetric mode in some cases of ECDF. For instance, Kreek [KRE93] saw evidence for small symmetric components in the mass-yield distributions from ECDF of ${ }^{228} \mathrm{~Np}$ and ${ }^{238} \mathrm{Bk}$. In the case of ${ }^{238} \mathrm{Bk}$, it was not certain whether or not there was indeed a symmetric mode because the statistics of the data for 
symmetric mass division were very poor. Hall saw no evidence of any symmetric components in the ECDF of ${ }^{232} \mathrm{Am}$ and ${ }^{234} \mathrm{Am}$ [HAL89].

According to the static fission model of Wilkins et al. [WIL76], actinides with neutron number greater than 140 should have asymmetric mass splits until the fermium region is reached $\left({ }^{242} \mathrm{Cf}\right.$ has $\mathrm{N}=144$.) This model is based on the assumption that the distribution of fission fragments can be determined at or near the scission point from the relative potential energies of the complementary fission fragment pairs. The fragment potential energies are calculated as functions of neutron number, proton number and deformation parameter. The total potential energy of the system at scission is calculated as the sum of liquid-drop and shell correction terms for two nearly touching, coaxial spheroids. In addition, an approximation is included for the surface energy of the neck connecting the two spheroids, and Coulomb and nuclear potential terms describe the interaction between the spheroids. For additional details about the model the reader is referred to [WIL76] and the references therein. According to Wilkins et al., the heavy fragment in the split remains constant around either the spherical neutron shell at $\mathrm{N}=82$ or the deformed neutron shell at $\mathrm{N} \approx 88$ for a wide range of $\mathrm{Z}$ and $\mathrm{A}$ of the fissioning nucleus. If the heavy fragment is located at the spherical neutron shell then the complement is forced by liquid-drop considerations to be highly deformed (most of the energy of the system is contained in the liquid-drop terms, not in shell effects.) Another aspect of the model is that there is a strong tendency arising from the liquid-drop terms for the fission fragments to maintain the N/Z ratio of the fissioning nucleus [WIL 76]. In order to maintain this ratio in ${ }^{242} \mathrm{Cf}(\mathrm{N} / \mathrm{Z}=1.47)$, the heavy fission fragment $(\mathrm{A}=138$, see Table . 6.1) would have to be just outside of the spherical shell with $N=82$ ( $Z=56, \beta=0.2$ where $\beta$ 
is the nuclear deformation taken from [WIL76].) The fragment would not have $\beta=0$ because a proton number of 56 has a minimum in the potential energy surface at $\beta=0.2$ [WIL76]. The complementary fragment would therefore be highly deformed with $\mathrm{N}=62$ $\left(\mathrm{Z}=42, \beta \approx 0.85\right.$.) If ${ }^{242} \mathrm{Cf}$ were to split symmetrically, both fragments would have $\mathrm{Z}=49$ and $N=72$. At first glance, it seems that proximity to the $Z=50$ spherical proton shell would promote two spherical fission fragments, resulting in a visible symmetric component in the fission of ${ }^{242} \mathrm{Cf}$. As seen in Figure 6.2, there was no symmetric component in the mass-yield distribution. The deformation diagrams in [WIL76] show that a neutron number of 72 is not close to any of the neutron shells and therefore prefers a deformation greater than $\beta=0.25$. The energy gained from liquid-drop considerations (maintaining the N/Z ratio of the parent) more than compensates for the loss in energy from moving out of the $Z=50$ shell [WIL76]. This brings the protons out of the spherical shell resulting in a fragment with a larger deformation. A symmetric split would therefore consist of two deformed fragments, resulting in an overall lower TKE than in the case of one nearly spherical fragment and one highly deformed fragment. This is consistent with the highly asymmetric mass-yield distribution seen in Figure 6.2 and the absence of a symmetric fission mode.

\subsubsection{Search for $\alpha$-Decay in ${ }^{242}$ Es}

As mentioned in the previous section, three separate experiments were performed in the study of ECDF in ${ }^{242}$ Es. The nuclides we were searching for in the $\alpha$-spectra from these experiments included ${ }^{242} \mathrm{Es}$ and its EC-daughter, ${ }^{242} \mathrm{Cf}$. (Table 3.2 gives decay properties of the einsteinium and californium nuclei discussed from this point on.) The 
first experiment used a 10-s stepping time in the $\mathrm{MG}$ (see Chapter 5) and a ${ }^{14} \mathrm{~N}$-beam energy of $84 \mathrm{MeV}$, which corresponded to the peak energy of the $5 \mathrm{n}$ exit channel according to the SPIT code [SIK67]. (see Figure 4.5). Figure 6.4 shows a typical $\alpha$ spectrum recorded during one of these ECDF experiments. Two problems were observed in the subsequent data. First of all ${ }^{243}$ Es was produced at nearly the same rate as ${ }^{242}$ Es. Looking at Figure 4.5, at an energy of $84 \mathrm{MeV}$ the production cross sections for the $4 \mathrm{n}$ and $5 \mathrm{n}$ exit channels are expected to be practically identical. The half-life and $\alpha$-energy of ${ }^{243}$ Es are very close to those of ${ }^{242}$ Es making it impossible to distinguish between these two isotopes in the $\alpha$-peak. A two-component half-life analysis of the $\alpha$-peak was attempted, but the two separate half-lives could not be identified. The other problem can be seen in Figure 6.4. ${ }^{213} \mathrm{Fr}\left(\mathrm{t}_{1 / 2}=34.6 \mathrm{~s}\right.$ [FIR96]) was made in such large quantities that it completely obscured the energy region where ${ }^{242} \mathrm{Cf}$ would appear. Francium and other interfering activities were produced by the interaction of the ${ }^{14} \mathrm{~N}$ beam with lead impurities in the target, which formed decay chains of actinium, francium, radon, radium, astatine and polonium isotopes. Even though the ${ }^{233} \mathrm{U}$ target material had been chemically treated to remove lead, the conditions of the glove box where the target preparation took place were such that avoiding lead completely during the chemical separation was impossible. Notice in Figure 6.4 that the scale of the ordinate changes between the ${ }^{213}$ Fr peak and the remainder of the spectrum. If the scale were kept constant throughout the entire spectrum, only the francium peak would be visible and all of the other peaks would be too small to see. To try and suppress the production of ${ }^{243}$ Es the second ECDF experiment used a higher beam energy of $89 \mathrm{MeV}$, which should have 


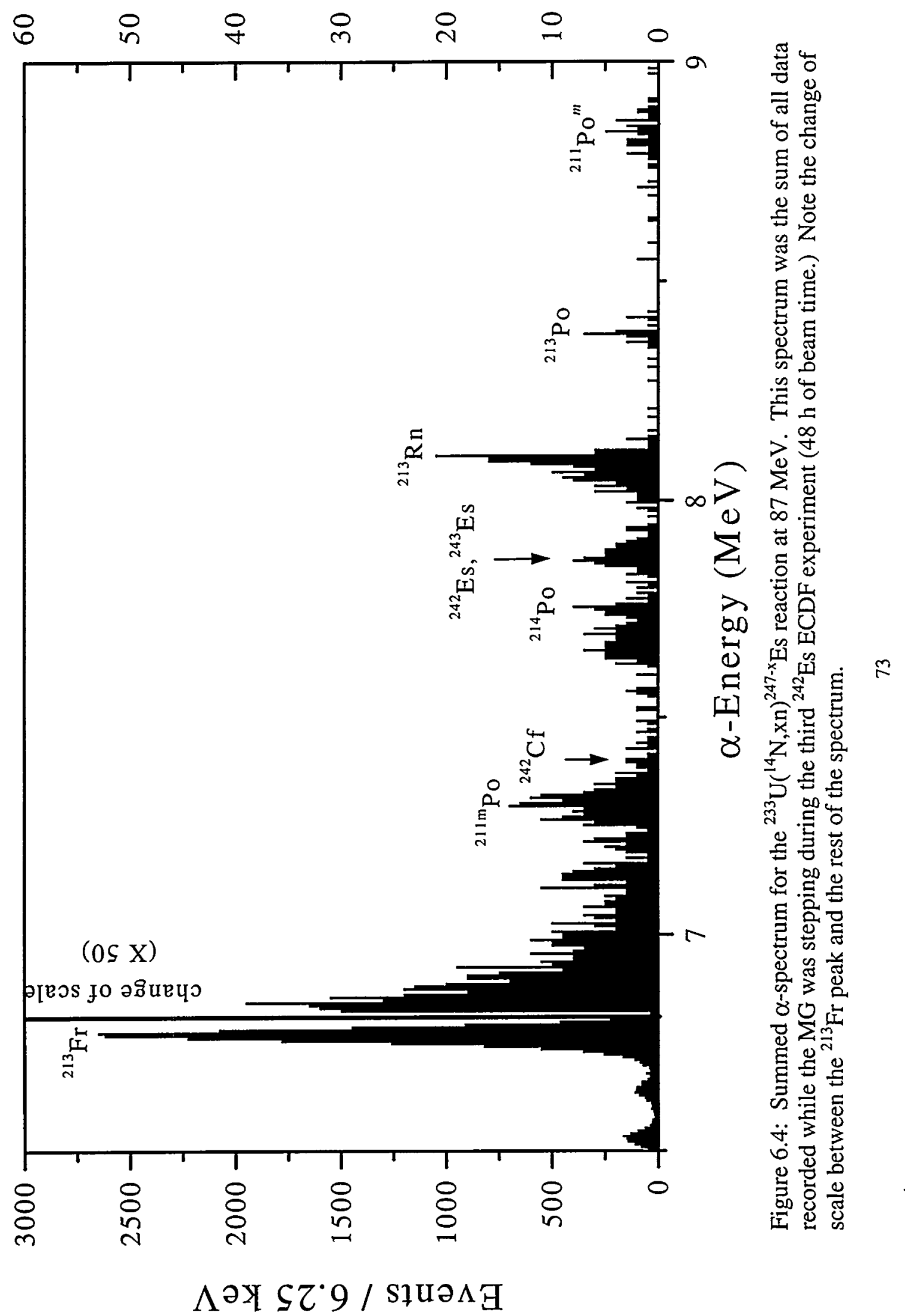


lowered the production of the $4 \mathrm{n}$ product in comparison to the $5 \mathrm{n}$ product by a factor of three. Even at this higher beam energy, a half-life analysis showed that ${ }^{243}$ Es still dominated the region where ${ }^{242}$ Es would have appeared. A two-component half-life analysis was attempted on the subsequent $\alpha$-peak, but again only one half-life was identified and the two isotopes could not be separated. Running at this higher beam energy also did not address the issue of the interfering ${ }^{213}$ Fr peak.

The third and final ECDF experiment used a different approach. It was decided that looking for $\alpha$-particles from ${ }^{242}$ Es using the MG was nearly impossible. According to Figure $4.5,{ }^{243}$ Es would always be produced no matter what beam energy was used. During the previous two experiments fission fragments had been detected, but in order to determine the $\mathrm{P}_{\mathrm{DF}}$, it was imperative to know how many ${ }^{242}$ Es EC-decays had occurred. For the third experiment, we wanted to accomplish two things, namely maximize the number of fissions produced and determine the total number of EC-decays. Based on the results of an excitation function experiment, which will be discussed in a later section, the beam was run at $87 \mathrm{MeV}$ to maximize the number of fissions detected. Because the search for ${ }^{242}$ Es $\alpha$-particles was no longer one of the main objectives of the experiment, the production of ${ }^{243}$ Es was not an issue. The MG was changed to 20 -s steps in order to spread the detection over a longer time interval. Finally, a new counting procedure was used with the MG. After the wheel collected and counted samples for $1 \mathrm{~h}$ (two and a quarter revolutions) the rotation was stopped so that the last six collections were still positioned under the six detector pairs. These six collections were then counted for an additional 40 min while the wheel was stationary. By counting while the wheel was not stepping we were able to look for the longer-lived ${ }^{242} \mathrm{Cf}$ over a longer time interval after 
the francium and other shorter-lived activities had decayed. Figure 6.5 shows a sample spectrum measured when the wheel was not stepping. The ${ }^{242} \mathrm{Cf}$ peak was still relatively small, but, because there was no longer such a huge ${ }^{213} \mathrm{Fr}$ background, we were able to identify the peak based on its half-life and $\alpha$-energy. Since ${ }^{242} \mathrm{Cf}$ decays solely by $\alpha-$ emission [FIR96], the number of ${ }^{242} \mathrm{Cf} \alpha$-particles we detected during the experiment was equivalent to the total number of ${ }^{242}$ Es EC-decays after taking the detection efficiency into account. The detection of ${ }^{242} \mathrm{Cf}$ during the third ${ }^{242} \mathrm{Es}$ ECDF experiment finally enabled us to determine the total number of EC-decays and, therefore, the $\mathrm{P}_{\mathrm{DF}}$.

The EC to $\alpha$-branching ratio of ${ }^{242}$ Es is unknown, and could not be determined from the ECDF experiments because of the interfering ${ }^{243}$ Es peaks. However, using $\alpha$-particles from ${ }^{242} \mathrm{Cf}$ as an indication of the number of ${ }^{242} \mathrm{Es} \mathrm{EC}$-decays, we were able to set a lower limit on the production cross section of the ${ }^{233} \mathrm{U}\left({ }^{14} \mathrm{~N}, 5 \mathrm{n}\right){ }^{242} \mathrm{Es}$ reaction. Assuming a $100 \%$ EC-branch in ${ }^{242}$ Es the lower limit of the cross section is $22 \mathrm{nb}$. Since Ninov et al. [NIN96] reported $\alpha$-particles from the decay of ${ }^{242}$ Es, the assumption of a 100\% EC-branch is not correct, but enables us to set the lowest possible value of the production cross section. A lower EC-branch results in a larger cross section. The SPIT code [SIK67] predicts a 32-nb cross section for this reaction at $87 \mathrm{MeV}$, and based on our value, the predicted value seems to be the correct order of magnitude. If the EC-branch is determined during a later experiment, then this cross section can be calculated more accurately.

By assuming that all of the 7.9-MeV $\alpha$-particles are from the decay of ${ }^{242} \mathrm{Es}$ (neglecting any decay from ${ }^{243} \mathrm{Es}$ ) we can also set limits on the $\alpha$ - and EC-branches of 


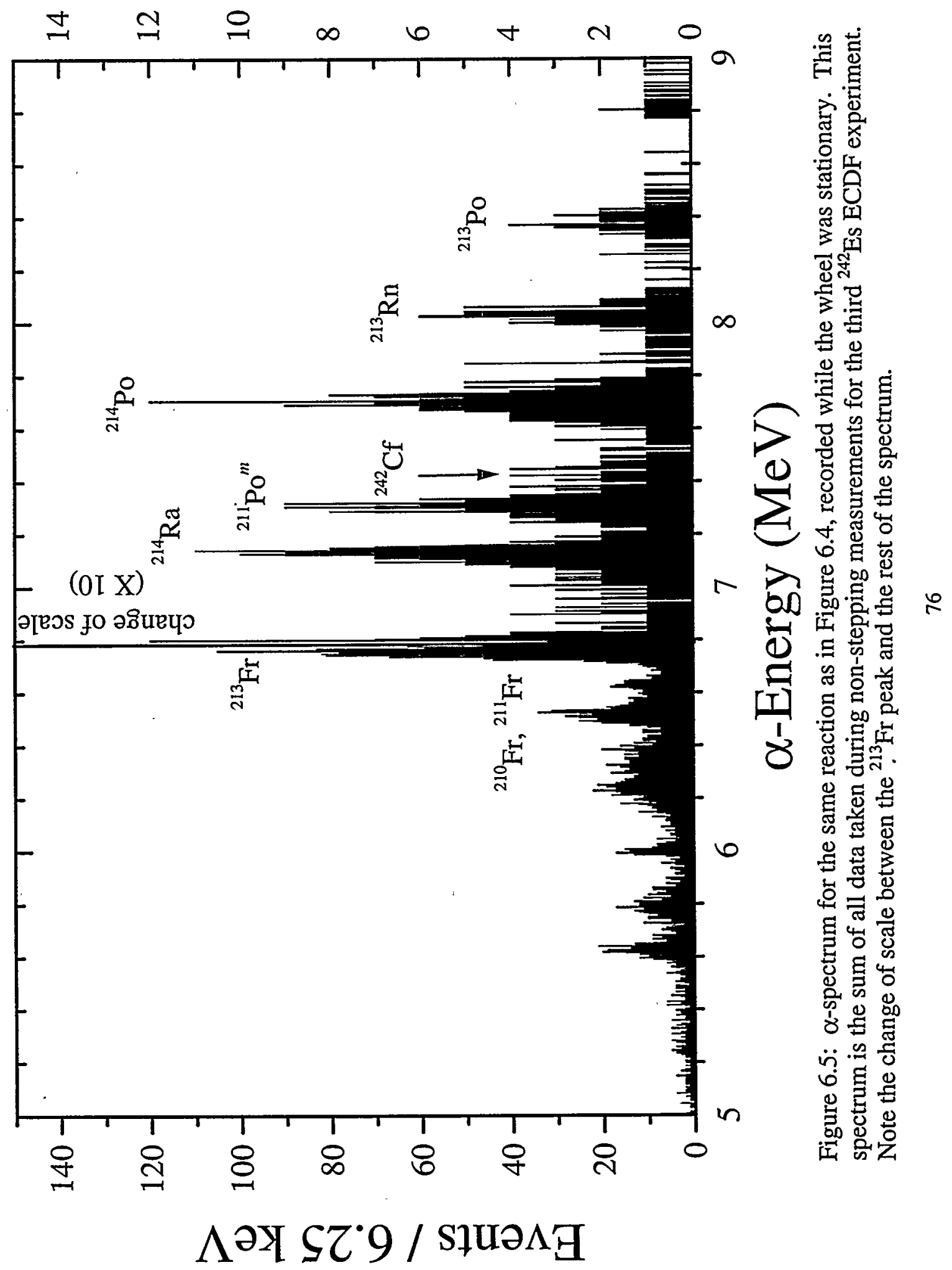


${ }^{242}$ Es. By comparing the number of 7.9-MeV $\alpha$-particles to the number of ${ }^{242} \mathrm{Cf} \alpha-$ decays, the upper limit of the $\alpha$-branch is approximately 0.25 and the lower limit of the EC-branch is approximately 0.75 . These numbers indicate that ${ }^{242}$ Es predominantly decays via EC, which may explain why a separate ${ }^{242}$ Es $\alpha$-peak was not observed in the spectra. The 7.9-MeV $\alpha$-peak observed in Figure 6.4 may have been almost entirely due to the $\alpha$-decay of ${ }^{243}$ Es with little contribution from ${ }^{242}$ Es.

The direct production of ${ }^{242} \mathrm{Cf}$ via the ${ }^{233} \mathrm{U}\left({ }^{14} \mathrm{~N}, \mathrm{p} 4 \mathrm{n}\right){ }^{242} \mathrm{Cf}$ reaction was neglected because of its low cross section. As discussed in Chapter 1, deexcitation of the compound nucleus via neutron emission is more probable than charged particle emission because protons must first overcome the Coulomb barrier in order to be emitted whereas neutrons do not. Even in cases where the $\mathrm{CN}$ is neutron-deficient, such as ${ }^{247}$ Es, neutron emission is still the preferred decay mode [KRA88]. Based on arguments presented in [GAN80] and [HEN90], we estimated that the p4n exit channel was less than $10 \%$ of the 5n exit channel, well within the standard deviation of our measurement.

\subsubsection{3 $\mathrm{P}_{\mathrm{DF}}$ and $\log f t$ Values}

The $\mathrm{P}_{\mathrm{DF}}$ of ${ }^{242}$ Es was determined from the third ECDF experiment because it was the only one sensitive to the detection of ${ }^{242} \mathrm{Cf} \alpha$-particles. The number of ${ }^{242} \mathrm{Cf}$ decays was determined by integrating a relatively narrow energy region from $7.370 \mathrm{MeV}$ to 7.390 MeV in Figure 6.5. This narrow region was used to avoid any interference from the neighboring ${ }^{211} \mathrm{Po}^{m}$ peak, and covered $80 \%$ of the ${ }^{242} \mathrm{Cf} \alpha$-peak (see table 3.2.) Based on 36 fissions and $70{ }^{242} \mathrm{Cf} \alpha$-particles detected during the third experiment (these values were later normalized to the number of samples collected), a $P_{D F}$ of $0.006 \pm 0.002$ was 
calculated. Based on the relationship between $\mathrm{P}_{\mathrm{DF}}$ and $\mathrm{Q}_{\mathrm{EC}}$ shown in Figure 2.2 and a $Q_{E C}$ of $5.35 \mathrm{MeV}$ [MÖL95], our value for the $\mathrm{P}_{\mathrm{DF}}$ of ${ }^{242}$ Es seems reasonable. ${ }^{242}$ Es has the largest $P_{D F}$ of any system where ECDF has been studied, which is expected based on its larger Q-value. Our mean value of 0.006 is lower than the previously reported value of $0.014 \pm 0.008$ from [HIN85], but is based on more fission events. Within the quoted standard deviations, the two values are consistent with each other.

Based on the single-particle Nilsson diagrams [FIR96] and a predicted nuclear deformation $(\beta)$ of 0.208 for ${ }^{242}$ Es [MÖL95], the $99^{\text {th }}$ proton in ${ }^{242}$ Es is most probably assigned to the $7 / 2^{+}[633]$ level and the $143^{\text {rd }}$ neutron can either be assigned to the $5 / 2^{+}[622]$ or $1 / 2^{+}[631]$ level. Based on the predicted deformation [MÖL95], the neutron level is most likely $5 / 2^{+}[622]$, which combines with a $7 / 2^{+}[633]$ proton level to produce a nucleus with a spin and parity in the range of $1^{+}$to $6^{+}$. The most likely assignment for the ground state spin and parity of ${ }^{242} \mathrm{Es}$ is $1^{+}$, resulting in an EC decay of $\Delta \mathrm{J}^{\pi}=1^{\text {no }}$ (assuming a ground state to ground state EC-transition.) An angular momentum change of one unit with no change in parity is normally an allowed transition. Actinides typically undergo fast forbidden transitions with $\log f t$ values of approximately six or greater [HAL89]. Using the diagrams in [FIR96] and assuming a 100\% EC-branch in ${ }^{242} \mathrm{Es}$, one obtains a $\log f t$ of 4.5 for the ${ }^{242} \mathrm{Es}$ to ${ }^{242} \mathrm{Cf} \mathrm{EC}$-transition. This is very fast, even for a typical allowed transition in the actinides, which normally has a $\log f t$ value in the range of 5.0 to 7.0 (approximately). As the EC-branch decreases, the corresponding $\log f t$ value increases. For example, a $\log f t$ of 5.0 would result in an EC-branch of $32 \%$ in ${ }^{242}$ Es. Based on previous experiments where the $5 n$ exit channel was measured in the production of neutron-deficient Cf, Fm and No isotopes ([SIK68], [SIK68A], and 
[NUR67],) the SPIT code either exactly matched the experimentally determined cross sections or was within a factor of five. $160 \mathrm{nb}$ would be a factor of five greater than the predicted value of $32 \mathrm{nb}$ for this reaction. To achieve this value, the EC-branch would have to be on the order of $23 \%$, resulting in a $\log \mathrm{ft}$ value of 5.3 , which is still within the range for an allowed transition in the actinides. However, as mentioned previously, we measured a lower limit of approximately $75 \%$ for the EC-branch, which implies that the $\log f t$ value falls within a very narrow range from 4.5 to approximately 4.7 , indicative of an allowed transition.

\section{$6.1 .2{ }^{244}$ Es}

\subsubsection{The ${ }^{233} \mathrm{U}\left({ }^{15} \mathrm{~N}, 4 \mathrm{n}\right){ }^{244}$ Es Reaction}

The first attempt to study ECDF in ${ }^{244} \mathrm{Es}$ used the ${ }^{233} \mathrm{U}\left({ }^{15} \mathrm{~N}, 4 \mathrm{n}\right){ }^{244} \mathrm{Es}$ reaction with a single target as described in Chapter 4. Originally, the ${ }^{15} \mathrm{~N}^{4+}$ beam was at $81 \mathrm{MeV}$, which corresponded to the maximum of the ${ }^{233} \mathrm{U}\left({ }^{15} \mathrm{~N}, 4 \mathrm{n}\right){ }^{244}$ Es reaction according to the SPIT [SIK67] code (see Figure 4.6.) The production cross section at this energy was predicted to be $1.5 \mu \mathrm{b}$, which would result in an EC-rate of 7152 events per hour for

${ }^{244}$ Es. Based on the previous estimate of $10^{-4}$ for the $P_{D F}$ of ${ }^{244}$ Es [GAN80], we expected to see approximately 15 delayed fission events per day, and hoped to determine a more accurate value for the $P_{D F}$.

No fission events were observed during the first $4 \mathrm{~h}$ of measurements. The rates of $\alpha$-events in the detectors were less than expected based on the predicted $1.5-\mu b$ cross section. Previous experience had shown that the SPIT code sometimes overestimated the magnitude and position of the $4 \mathrm{n}$ exit channel in various reactions [GRE96A], so it was 
speculated that the maximum of the $4 \mathrm{n}$ channel might be at a higher energy. The beam energy was therefore raised to $86 \mathrm{MeV}$ to try and increase production of ${ }^{244} \mathrm{Es}$. According to SPIT, the cross section at $86 \mathrm{MeV}$ would be approximately $1 \mu \mathrm{b}$ (see Figure 4.6.)

A total of $10 \mathrm{MG}$ wheels were measured at the higher beam energy for a total of $10 \mathrm{~h}$ of counting and only two coincident fission events were detected during that time instead of the seven or so we had expected. At that point the experiment was terminated because it was evident that the production of ${ }^{244} \mathrm{E}$ s was less than what was originally anticipated, and the predicted cross section must therefore be incorrect. From the $\alpha-$. spectra it was observed that ${ }^{244} \mathrm{Es}$ had been produced in the reaction, but not at the rate we had originally calculated. Figure 6.6 shows a typical $\alpha$-spectrum from the ${ }^{233} \mathrm{U}\left({ }^{15} \mathrm{~N}, \mathrm{xn}\right){ }^{248-\mathrm{x}} \mathrm{Es}$ reaction for all of the runs summed together (both beam energies included.) Several interfering activities produced from the interaction of the beam with lead impurities in the target (see section 6.1.1.2 for more details about these activities) were also observed in the spectra. ${ }^{244} \mathrm{Cf}$, the EC-daughter of ${ }^{244} \mathrm{Es}$, only appeared as a small shoulder on the much larger ${ }^{211} \mathrm{Po}^{m}$ peak.

Based on $14{ }^{244} E s \alpha$-particles measured in the top detector of the second detector pair over the course of the experiment and a ${ }^{244}$ Es $\alpha$-branch of $4 \%$ [FIR96], a production cross section of $73 \pm 54 \mathrm{nb}$ was determined for the ${ }^{233} \mathrm{U}\left({ }^{15} \mathrm{~N}, 4 \mathrm{n}\right){ }^{244} \mathrm{Es}$ reaction at $86 \mathrm{MeV}$. SPIT predicted a cross section that was approximately 14 times higher than what we measured, and this smaller cross section resulted in a lower production rate of ${ }^{244} \mathrm{Es}$ and fewer fission events than anticipated. Based on these results, we decided to try another 


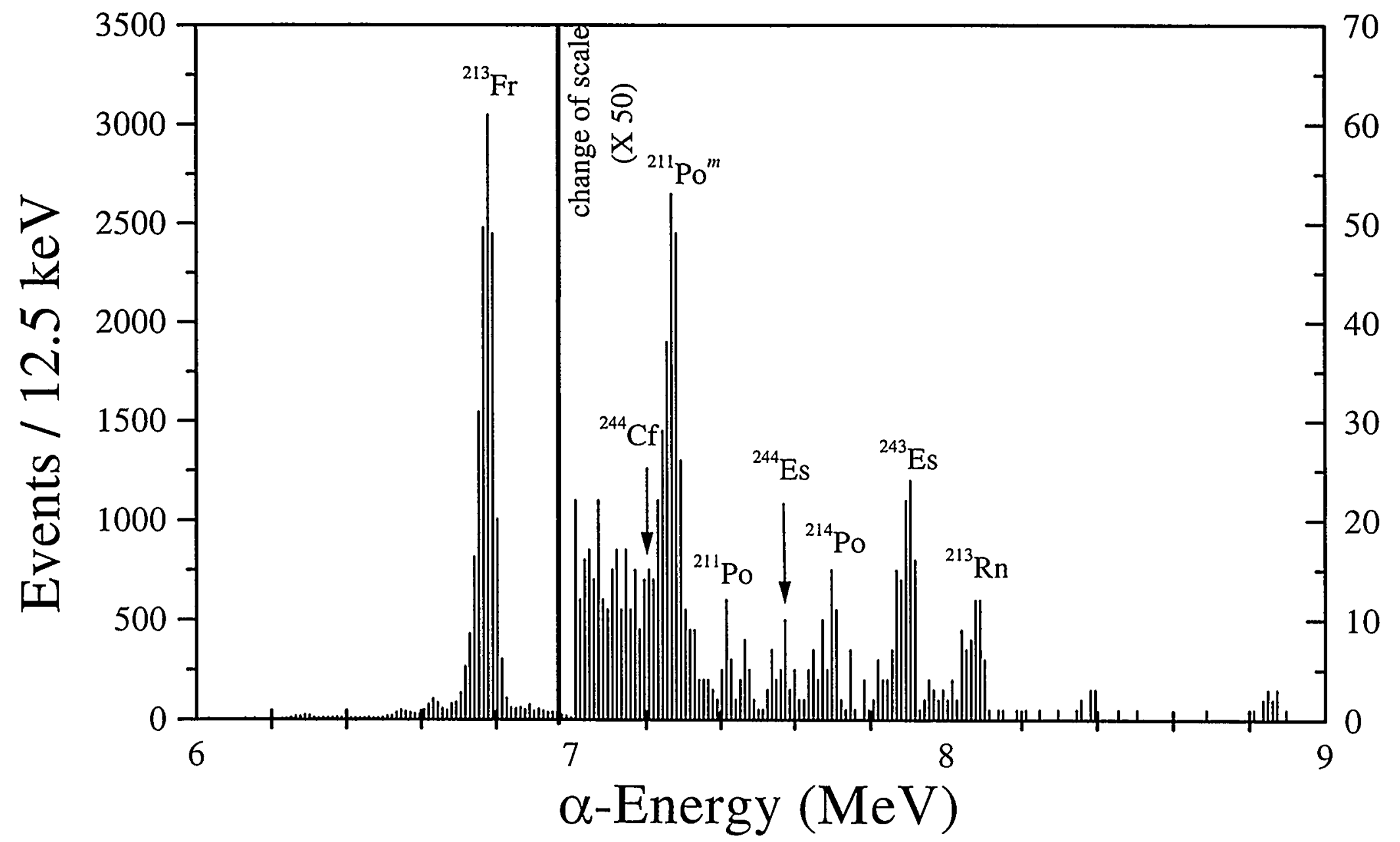

Figure 6.6: Summed $\alpha$-spectrum from the ${ }^{233} \mathrm{U}\left({ }^{15} \mathrm{~N}, \mathrm{xn}\right){ }^{248-\mathrm{x}}$ Es reaction at energies of 81 and $86 \mathrm{MeV}$. Note the change of scale after the ${ }^{213} \mathrm{Fr}$ peak. This spectrum was from all of the runs summed together (approximately $15 \mathrm{~h}$ of beam time) and was taken from detector 2-top. 
reaction for producing ${ }^{244} \mathrm{Es}$, which would have a larger production rate resulting in more fission events.

\subsubsection{The ${ }^{237} \mathrm{~Np}\left({ }^{12} \mathrm{C}, 5 \mathrm{n}\right){ }^{244}$ Es Reaction}

\subsection{Fission Properties}

Hatsukawa et al. [HAT89] had used the ${ }^{237} \mathrm{~Np}\left({ }^{12} \mathrm{C}, 4 \mathrm{n}\right)$ reaction to produce ${ }^{245} \mathrm{Es}$. In their spectra they also observed ${ }^{244} \mathrm{Cf}$, the EC-daughter of ${ }^{244} \mathrm{Es}$, which was produced in the reaction. According to the SPIT code [SIK67], ${ }^{244}$ Es should be produced in this reaction with a predicted maximum cross section of approximately $1.5 \mu \mathrm{b}$ at $81 \mathrm{MeV}$ (see Figure 4.7.) Previous experiments have shown that the SPIT code is often more accurate in predicting $5 \mathrm{n}$ exit channel cross sections than in predicting $4 \mathrm{n}$ cross sections. It was speculated that the predicted cross section of $1.5 \mu \mathrm{b}$ for this $5 \mathrm{n}$ reaction might be closer to the actual cross section than the prediction for the ${ }^{233} \mathrm{U}\left({ }^{15} \mathrm{~N}, 4 \mathrm{n}\right)$ reaction, resulting in more fission events than the previous experiment. We therefore decided to produce ${ }^{244}$ Es using the ${ }^{237} \mathrm{~Np}\left({ }^{12} \mathrm{C}, 5 \mathrm{n}\right)$ reaction at $81 \mathrm{MeV}$.

A stepping time of $30 \mathrm{~s}$ was used in the $\mathrm{MG}$ and each wheel underwent two complete revolutions, corresponding to an 80-min collection and measurement time. After that time, the last six samples of each wheel were counted while the wheel was stationary for an additional $40 \mathrm{~min}$ so that the longer-lived ${ }^{244} \mathrm{Cf}$ could be detected after most of the interfering activities had decayed away. In the previous ${ }^{244}$ Es experiment, ${ }^{244} \mathrm{Cf}$ could not be measured because of the much larger ${ }^{211} \mathrm{Po}^{m}$ peak observed in the spectra (see Figure 6.6.) Counting the wheel while it was stationary allowed the 
polonium peak to decay so the ${ }^{244} \mathrm{Cf}$ could be measured, enabling us to determine the $\mathrm{P}_{\mathrm{DF}}$ of ${ }^{244}$ Es (see section 6.1.2.2.3.)

A total of 16 coincident fission events were detected over the course of the experiment ( $36 \mathrm{~h}$ of beam time.) A background measurement taken before the experiment indicated that less than one fission event in all of the detectors could be attributed to long-lived background activities. Analysis of the data showed that sometime during the experiment the first detector pair had ceased working properly. Only two coincident fission events were detected in the first detector pair instead of the approximately 10 we expected based on the subsequent decay curve of fission events from the other detectors. It was possible that even more fission events were produced during the experiment, but we were unable to detect them because of this malfunction. The two events from detector pair one were removed from the decay curve analysis for ${ }^{244} \mathrm{Cf}$ based on coincident fissions, but were included in determining its fission properties. Figure 6.7 shows the decay curve of coincident fission events. A long-lived activity of 0.05 counts/s was observed in the decay curve and was included in the subsequent analysis (Figure 6.7 only shows the ${ }^{244}$ Es component.) The half-life of the fission events was determined using the MLDS code with a two-component fit. The shorter component had a half-life of $31 \pm 10 \mathrm{~s}$, which indicates that the fission events came from the delayed fission of ${ }^{244}$ Es since the fission events decay with the characteristic half-life of the parent nucleus (see section 6.1.1.1 for details.) The only other nuclide that could be produced in this reaction with a similar half-life is ${ }^{243}$ Es. It is unlikely that the fission events could have come from ${ }^{243}$ Es for two reasons. First, ${ }^{243} \mathrm{Es}$ would have been produced in a $6 \mathrm{n}$ reaction, which, according to Figure 4.7, would have a cross section two orders of 
magnitude lower than the $5 \mathrm{n}$ reaction resulting in a much lower production rate. Second, because of its lower $\mathrm{Q}_{E C}$ of $4.0 \mathrm{MeV}$ [MÖL95] the $\mathrm{P}_{\mathrm{DF}}$ of ${ }^{243} \mathrm{Es}$ based on systematics would be too low to account for the number of fissions detected during the experiment (see section 6.1.1.1 for more details on this point.) Therefore, the 16 fission events were attributed to the delayed fission of ${ }^{244}$ Es.

Fission energy calibrations were performed with a ${ }^{252} \mathrm{Cf}$ source as discussed in Chapter 5. The average neutron emission function of ${ }^{244} \mathrm{Cf}, \bar{v}(A)$, was assumed to be similar to that of ${ }^{252} \mathrm{Cf}$, normalized to an average neutron emission $\bar{v}_{t}=2.6$ (estimated from systematics in [HOFFM74].) Since fission events in ECDF are preceded by ECdecays, the fission properties measured during the experiment are for the EC-daughter, ${ }^{244} \mathrm{Cf}$. Figure 6.8 shows the mass-yield distribution of ${ }^{244} \mathrm{Cf}$. The highly asymmetric mass distribution is clearly double-humped and has a very deep valley, with no evidence of a symmetric component.

The pre-neutron total kinetic energy (TKE) distribution for coincident fission fragments from ${ }^{244} \mathrm{Cf}$ is shown in Figure 6.9. The average pre-neutron emission TKE was $186 \pm 19 \mathrm{MeV}$. Table 6.2 summarizes the mass and kinetic energy information from the $\mathrm{ECDF}$ of ${ }^{244}$ Es. Since the ${ }^{252} \mathrm{Cf}$ calibration source was on the same kind of polypropylene foil used on the MG wheel during these experiments, no correction was made for energy degradation of fission fragments through the foil en route to the bottom detectors. Also, no correction was made for the approximately $15 \mu \mathrm{g} / \mathrm{cm}^{2}$ of $\mathrm{KCl}$ aerosol [KRE94A] deposited on each foil by the gas-jet transport system because typical fission fragments only lose $0.2-0.4 \mathrm{MeV}$ of energy [FIR96] as they travel through this amount of $\mathrm{KCl}$ to the 


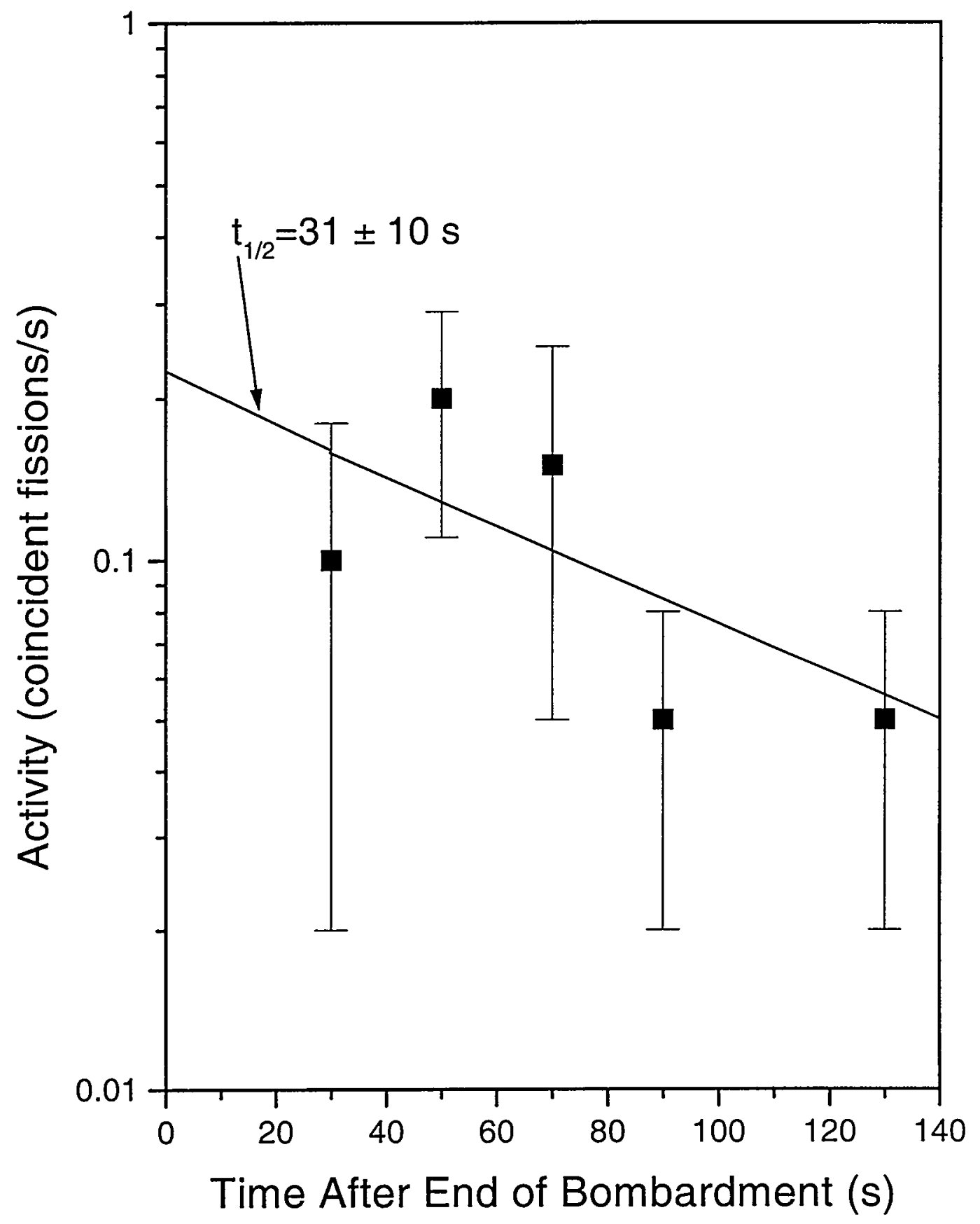

Figure 6.7: Decay curve of coincident fission events from the ECDF of ${ }^{244} \mathrm{Es}$ (detector pair one omitted.) Total running time was $36 \mathrm{~h}$. The line represents the best fit of the shorter ${ }^{244}$ Es component as determined by MLDS. 


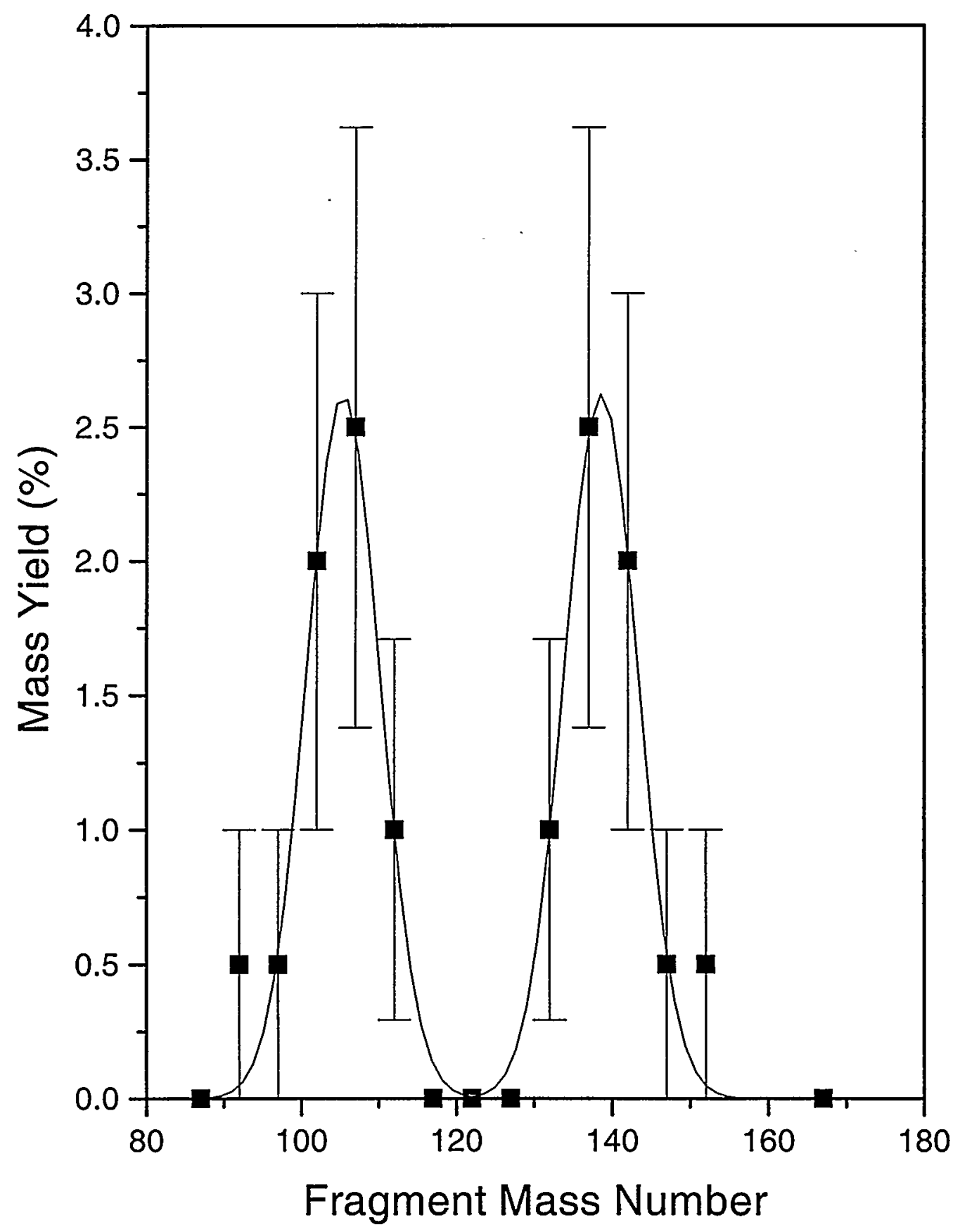

Figure 6.8: Pre-neutron emission mass-yield distribution for the ECDF of ${ }^{244}$ Es. The fissioning species is ${ }^{244} \mathrm{Cf}$. The data were averaged over 5 mass units. 


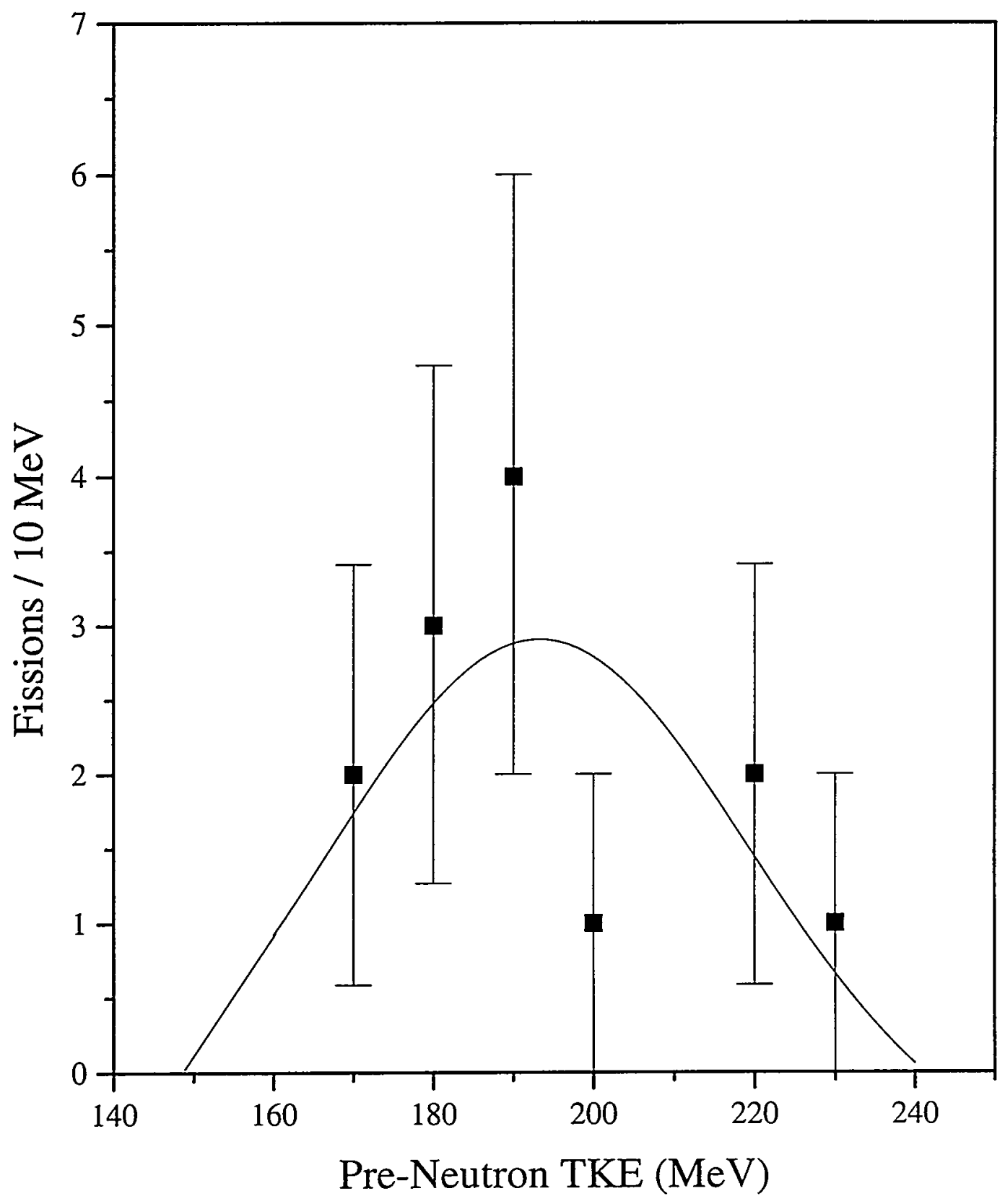

Figure 6.9: Pre-neutron emission total kinetic energy distribution (TKE) for the ECDF of ${ }^{244}$ Es. The line represents the Gaussian fit to the data. The data are in groupings of $10 \mathrm{MeV}$. 
detectors. The average TKE of $186 \pm 19 \mathrm{MeV}$ agrees within error with the empirical predictions of $193 \mathrm{MeV}$ and $190 \mathrm{MeV}$ made by Unik et al. [UNI73] and Viola et al. [VIO85], respectively. The difference between the average and most probable TKE values for ${ }^{244} \mathrm{Cf}$ in Table 6.2 is due to thick $\mathrm{KCl}$ deposits on the foils, resulting in poor sample quality and degradation of fission fragment energy. This was not observed in ${ }^{242} \mathrm{Cf}$ because a shorter stepping time was used, resulting in less $\mathrm{KCl}$ and better samples. According to the static fission model of Wilkins et al. [WIL76] discussed in section 6.1.1.1, the heavy fission fragment should remain constant around either the $\mathrm{N}=82$ (spherical) or $\mathrm{N}=88$ (deformed) neutron shells. Experiments have shown that as the mass of the fissioning species increases, the heavy mass fragment remains nearly constant in position and the light mass fragment shifts toward higher mass numbers [WIL76]. This trend is also observed in the californium isotopes. The most probable heavy fragment in the split of ${ }^{244} \mathrm{Cf}$ has a mass of 141 (see Table 6.2), similar to the value of 138 seen in ${ }^{242} \mathrm{Cf}$. Even with the addition of two neutrons, both the heavy and light fragments have remained nearly constant in position in going from ${ }^{242} \mathrm{Cf}$ to ${ }^{244} \mathrm{Cf}$. $\mathrm{As}$ in the case of ${ }^{242} \mathrm{Cf}$, the heavy fragment in the fission of ${ }^{244} \mathrm{Cf}$ is just outside the spherical neutron shell with $N=82(Z=56, \beta=0.2$ where $\beta$ is the nuclear deformation taken from [WIL76], ) and its complement is therefore highly deformed with $N=64(Z=39, \beta \approx 0.9)$. A symmetric split would result in two fragments with $\mathrm{Z}=49$ and $N=73$. The presence of the $Z=50$ spherical proton shell would normally suggest the existence of two spherical fragments, but there are no corresponding neutron shells around $N=73$, which means that the fragments would prefer deformations greater than 0.25 . The two fragments would instead be more deformed, resulting in a lower TKE than in the case of one nearly 
spherical fragment and one deformed fragment. This is consistent with the highly asymmetric mass-yield distribution seen in Figure 6.8 and the absence of a symmetric mode.

Table 6.2. Properties of the measured post-neutron-emission (Post-n) and calculated initial pre-neutron-emission (Pre-n) fragment kinetic energy and mass distributions for ${ }^{244} \mathrm{Es}$ ECDF and the ${ }^{252} \mathrm{Cf}$ standard measured in the same system. Once the ${ }^{252} \mathrm{Cf}$ preneutron-emission fragment energies are calculated, these values are used for calibration input parameters. Energies are given in $\mathrm{MeV}$, based on the SKW [SCH65] calibration method with the Weissenberger constants [WEI86].

\begin{tabular}{|lcccc|}
\hline & \multicolumn{2}{c}{${ }^{244}$ Cf ECDF } & \multicolumn{2}{c|}{${ }^{252}$ Cf Standard } \\
& Pre-n & Post-n & Pre-n & Post-n \\
\hline Average TKE & 186 & 184 & 181 & 179 \\
$\sigma$ & 19 & 18 & 16 & 15 \\
Most probable TKE & 193 & 190 & 182 & 181 \\
$\sigma$ & 20 & 17 & 16 & 15 \\
FWHM $^{\text {a }}$ & 47.0 & 40.0 & 37.6 & 35.3 \\
Light fragment energy $^{b}$ & 78.6 & 78.0 & 78.3 & 77.5 \\
$\sigma$ & 10.4 & 10.8 & 10.3 & 9.2 \\
Heavy fragment energy $^{\mathrm{b}}$ & 107 & 106 & 103 & 102 \\
$\sigma$ & 10 & 10 & 9 & 9 \\
Light fragment mass $^{\mathrm{c}}$ & 103 & N/A & 108 & N/A \\
Heavy fragment mass $^{c}$ & 141 & N/A & 144 & N/A \\
\hline
\end{tabular}

${ }^{\mathrm{a}}$ Full width at half maximum, calculated from $2.35 \sigma$ for Gaussian fit to the top half of the peak.

${ }^{6}$ These represent most probable values.

${ }^{\mathrm{c}}$ Masses calculated from most probable pre-neutron energies.

\subsubsection{2 $\alpha$-Decay in ${ }^{244}$ Es}

Figure 6.10 shows the summed $\alpha$-spectrum taken from all of the MG wheels measured during the experiment in the first top detector ( $36 \mathrm{~h}$ of beam time.) The interfering activities in the spectrum arise from the interaction of the ${ }^{12} \mathrm{C}$ beam with lead impurities in the target. The combination of lead and ${ }^{12} \mathrm{C}$ projectiles produces radium isotopes that decay with very short half-lives, producing isotopes of francium and 


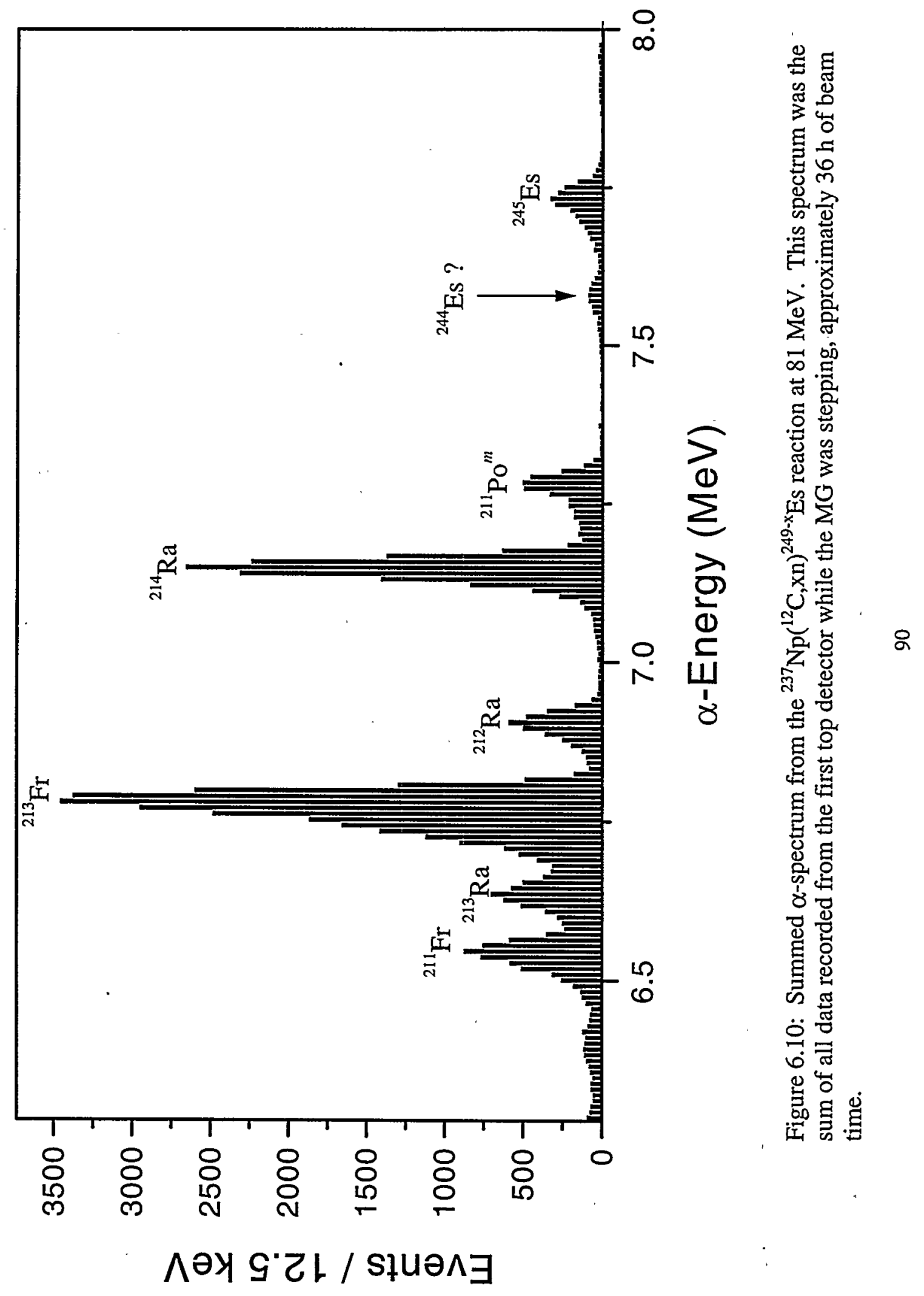


polonium. Also seen in the spectrum is ${ }^{245} \mathrm{Es}$, which is produced via the ${ }^{237} \mathrm{~Np}\left({ }^{12} \mathrm{C}, 4 \mathrm{n}\right){ }^{245}$ Es reaction. The $4 \% \alpha$-branch of ${ }^{244} \mathrm{Es}$ [FIR96] was originally observed in the $\alpha$-spectra from the ${ }^{233} U\left({ }^{15} \mathrm{~N}, 4 \mathrm{n}\right){ }^{244}$ Es reaction (see section 6.1.2.1), so it was assumed that it would be observed in these spectra as well. A peak was seen at 7.580 $\mathrm{MeV}$ and was first thought to be ${ }^{244} \mathrm{Es}$, but a half-life analysis of the peak using the MLDS code resulted in a half-life of approximately $1 \mathrm{~min}$. A two-component fit to the peak resulted in half-lives close to $1 \mathrm{~min}$ and less than $1 \mathrm{~s} .{ }^{244}$ Es might be in this 7.580 $\mathrm{MeV} \alpha$-peak, but with only a $4 \% \alpha$-branch it is obscured by the tail of the much larger ${ }^{245}$ Es peak, resulting in the apparent 1-min half-life. Since the half-life analysis did not result in a 37-s activity, the initial activity of the ${ }^{244}$ Es could not be determined from the $\alpha$-spectra. To determine the $\mathrm{P}_{\mathrm{DF}}$ and production cross section, we looked instead to the spectra recorded while the wheel was stationary to identify ${ }^{244} \mathrm{Cf}$, the EC-daughter of ${ }^{244}$ Es.

Figure 6.11 shows the summed spectrum of all measurements made while the wheel was stationary from the sixth top detector (approximately $13 \mathrm{~h}$ of counting.) The sample in detector pair six had the longest delay between collection and the start of counting (150 s), which allowed most of the short-lived interfering activities to decay before data were recorded. ${ }^{244} \mathrm{Cf}$ is clearly seen without any interfering activities. The longer-lived radon isotopes in the spectrum are from the decay of radium isotopes seen in Figure 6.10. Since ${ }^{244} \mathrm{Cf}$ decays solely via $\alpha$-emission, the number of counts in the corresponding $\alpha$-peak is equal to the number of ${ }^{244}$ Es EC-decays. A small correction was made to account for the $4 \% \alpha$-branch in ${ }^{244}$ Es [FIR96]. Based on this number of ${ }^{244}$ Es EC-decays, the $\mathrm{P}_{\mathrm{DF}}$ was calculated (see section 6.1.2.2.3 for details.) 


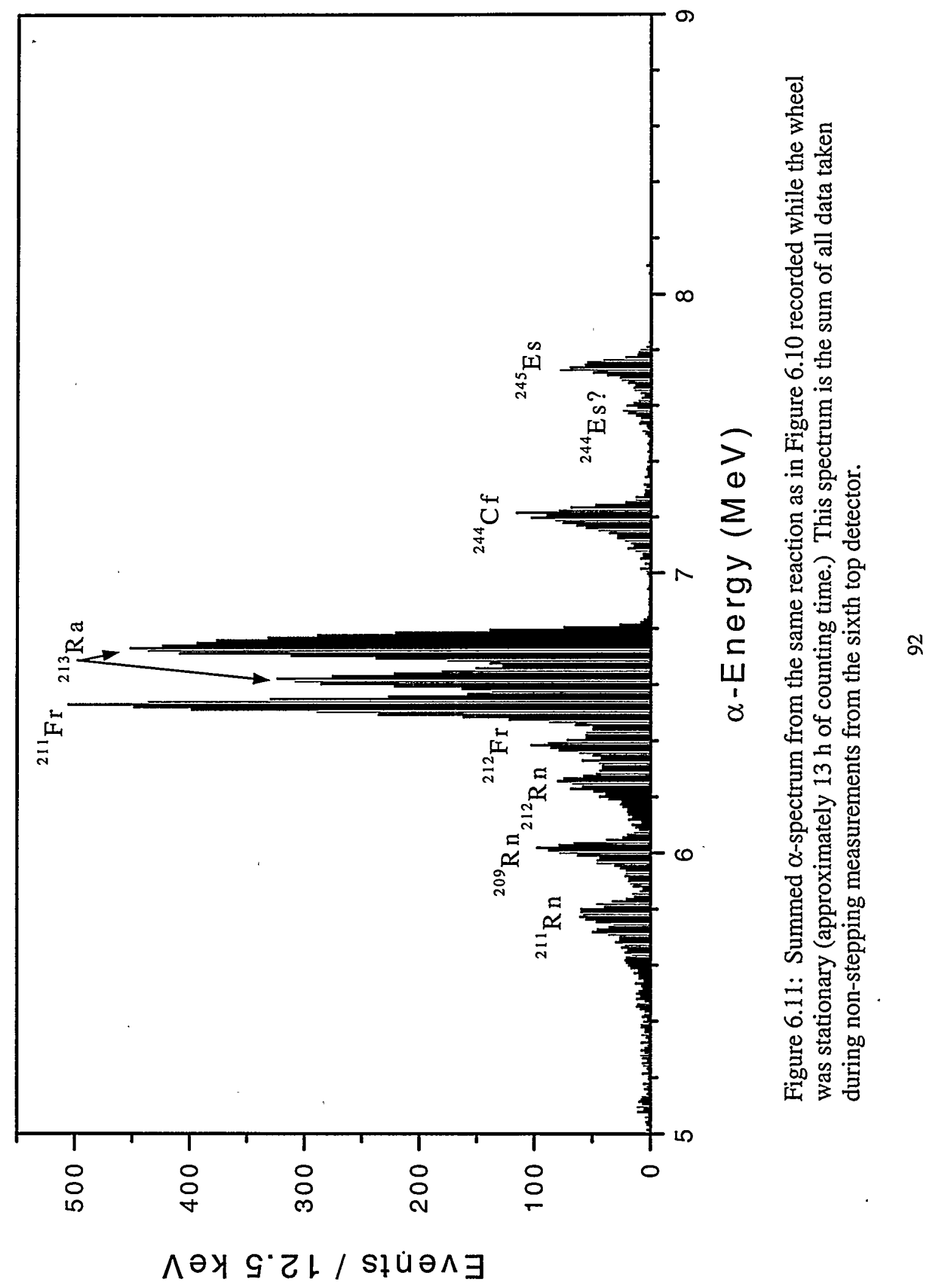


From the number of ${ }^{244}$ Es EC-decays, we also determined a cross section of 0.31 $\pm 0.12 \mu \mathrm{b}$ for the ${ }^{237} \mathrm{~Np}\left({ }^{12} \mathrm{C}, 5 \mathrm{n}\right){ }^{244}$ Es reaction at $81 \mathrm{MeV}$. This value is about a factor of five lower than the SPIT prediction of $1.5 \mu \mathrm{b}$ (see Figure 4.7), but is still more than a factor of four higher than the 73-nb cross section determined for the ${ }^{233} \mathrm{U}\left({ }^{15} \mathrm{~N}, 4 \mathrm{n}\right){ }^{244} \mathrm{Es}$ reaction (see section 6.1.2.1). This explains why so many more fission events were detected during this experiment than in the previous experiment using the ${ }^{233} \mathrm{U}$ target and ${ }^{15} \mathrm{~N}$ projectiles. As mentioned in section 6.1.1.3, the SPIT code [SIK67] predictions for the $5 n$ exit channel in the production of neutron-deficient actinides is usually correct within a factor of five. These experimental results are another example, showing that the code was able to predict the magnitude of the ${ }^{237} \mathrm{~Np}\left({ }^{12} \mathrm{C}, 5 \mathrm{n}\right)$ reaction with relative accuracy. The general ability of the code to predict specific cross sections will be discussed in more detail in section 6.2.4.2.

In determining the production cross section, the direct production of ${ }^{244} \mathrm{Cf}$ via the ${ }^{237} \mathrm{~Np}\left({ }^{12} \mathrm{C}, \mathrm{p} 4 \mathrm{n}\right){ }^{244} \mathrm{Cf}$ reaction was neglected because the cross section was assumed to be less than $10 \%$ of the $5 \mathrm{n}$ exit channel, well within the standard deviation of our measurement (see section 6.1.1.2). Most of the error associated with the calculated cross section arises from uncertainty in the yield of the gas-jet system.

\subsubsection{3 $\mathrm{P}_{\mathrm{DF}}$ and $\log f t$ Values}

The $P_{D F}$ of ${ }^{244} E s$ was determined from the number of delayed fissions and the $\alpha$ decay of ${ }^{244} \mathrm{Cf}$. An energy region from $7.080 \mathrm{MeV}$ to $7.270 \mathrm{MeV}$, which incorporates $100 \%$ of the ${ }^{244} \mathrm{Cf} \alpha$-particles, was integrated to determine the number of ${ }^{244} \mathrm{Cf} \alpha$-decays. Based on 20 single fission events (non-coincident) and $382{ }^{244} \mathrm{Cf} \alpha$-particles detected 
over the course of the experiment (these values were later normalized to the number of samples collected), a $\mathrm{P}_{\mathrm{DF}}$ of $(1.2 \pm 0.4) \times 10^{-4}$ was measured. Based on the relationship between $P_{D F}$ and $Q_{E C}$ shown in Figure 2.2 and a $Q_{E C}$ of $4.36 \mathrm{MeV}$ [MÖL95], our value for the $P_{D F}$ of ${ }^{244} E s$ seems reasonable. The value determined for ${ }^{244}$ Es falls roughly between ${ }^{234} \mathrm{Am}$ and ${ }^{228} \mathrm{~Np}$ as expected based on their respective $\mathrm{QEC}_{\mathrm{EC}}$ values (see Figure 2.2.) The only previously reported value for the $P_{D F}$ of ${ }^{244} E s$ was $10^{-4}$ estimated by Gangrskii et al. [GAN80]. Our value is consistent with their estimate, but is more precise.

Using the diagrams in [FIR96], a $\log f t$ of approximately 6.2 is obtained for the EC-transition of ${ }^{244}$ Es to ${ }^{244} \mathrm{Cf}$, based on a $4 \% \alpha$-branch in ${ }^{244}$ Es. A $\log f$ of approximately 6 is indicative of a first-forbidden transition in the actinides. Based on the single-particle Nilsson diagrams [FIR96] and a predicted nuclear deformation ( $\beta$ ) of 0.217 for ${ }^{244}$ Es [MÖL95], the $99^{\text {th }}$ proton in ${ }^{244}$ Es is most likely $7 / 2^{+}[633]$. The assignment of the $145^{\text {th }}$ neutron is unclear, since the single-particle levels change between nuclear deformations of 0.2 and 0.23 . At a deformation of 0.2 the neutron level is clearly $1 / 2^{+}[631]$. Somewhere between 0.2 and 0.23 this assignment changes to $7 / 2-[743]$. The predicted deformation of ${ }^{244}$ Es falls roughly between these two deformations so the assignment of the neutron level is not immediately obvious.

If the $145^{\text {th }}$ neutron were assigned to the $1 / 2^{+}[631]$ level and combined with the $7 / 2^{+}[633]$ proton level, then ${ }^{244}$ Es would have an assignment of $3^{+}$or $4^{+}$. The ground state to ground state EC-transition of ${ }^{244} \mathrm{Es}$ would require a spin and parity change of at least $\Delta \mathrm{J}^{\pi}=3^{\text {no }}$, which would be highly forbidden, resulting in a $\log f t$ greater than 10 . If the neutron were assigned to the 7/2[743] level, then the ${ }^{244}$ Es nucleus would be in the 
range of $0^{-}$to $7^{-}$. A ground state spin and parity assignment of $0^{-}$for ${ }^{244}$ Es would be the most likely, and the resulting ground state EC-decay would be $\Delta \mathrm{J}^{\pi}=0^{\text {yes }}$. This is a first-forbidden transition, and a $\log f t$ of 6.2 is reasonable for this type of decay. This indicates that $\beta$ of ${ }^{244}$ Es is greater than 0.2 as predicted and the $145^{\text {th }}$ neutron should be assigned to the 7/2-[743] level, resulting in a first-forbidden EC-transition with a $\log f t$ of approximately 6.2 .

\subsection{3 ${ }^{246}$ Es and ${ }^{248}$ Es}

\subsubsection{1 $\alpha$-Decay}

${ }^{246}$ Es and ${ }^{248}$ Es were produced via the ${ }^{249} \mathrm{Cf}(\mathrm{p}, \mathrm{xn}){ }^{250-\mathrm{x}}$ Es reaction using 19 thin ${ }^{249} \mathrm{Cf}$ targets in the LIM target system as described in Chapter 4. The proton beam was initially run at $37 \mathrm{MeV}$ to study ${ }^{246} \mathrm{Es}$ and was then shifted down to $18 \mathrm{MeV}$ for the study of ${ }^{248}$ Es. No correction was made for the difference between the energy of the beam out of the cyclotron and the desired energy on target because the energy lost by proton beams as they travel through the entire LIM system is negligible [NOR70]. During the experiment, it was noted that the first detector pair in the MG was contaminated with ${ }^{252} \mathrm{Cf}$, which had been used for fission energy calibrations prior to the start of the experiment (see Chapter 5). Any fission data collected from these detectors during the experiment were not included in later data analysis.

Only one MG wheel measured at $37 \mathrm{MeV}$ could be analyzed after the experiment because of a problem with the data acquisition system. Figure 6.12 shows the $\alpha-$ spectrum from this wheel recorded with the top detector from the second detector 
pair using a 1-min stepping time. Because of the longer stepping time, more $\mathrm{KCl}$ salt was deposited on the foils by the gas-jet transport system, worsening the $\alpha$-energy resolution. As the amount of salt on the foil increases, the $\alpha$-particles lose more energy as they pass through the salt en route to the detectors, creating a larger tailing effect than was seen in previous experiments. A larger salt deposit also means that more beta-active background activities are collected, and the piling up of beta-particles worsens the $\alpha$-resolution. The large $\alpha$-peak in the spectrum was integrated from $6.5 \mathrm{MeV}$ to $7.5 \mathrm{MeV} .{ }^{246} \mathrm{Es}$ and ${ }^{247} \mathrm{Es}$ have similar half-lives (7.7 $\mathrm{min}$ and $4.8 \mathrm{~min}$, respectively [FIR96]) and the two isotopes could not be resolved over a counting interval of only $6 \mathrm{~min}$. A two-component half-life analysis of the $7.3 \mathrm{MeV} \alpha$-peak was attempted using the MLDS code. The results were one component with a $4.2 \pm 0.1 \mathrm{~min}$ half-life $\left({ }^{247} \mathrm{Es}\right)$ and a longer component, which was a combination of ${ }^{246} \mathrm{Es},{ }^{245} \mathrm{Cf}$ and ${ }^{246} \mathrm{Cf}$. The initial $\alpha$-activity of ${ }^{247} \mathrm{Es}$ was calculated to be $1240 \pm 35$ counts $/ \mathrm{min}$, which translated into a production cross section of $11 \pm 7 \mu \mathrm{b}$ for the ${ }^{249} \mathrm{Cf}(\mathrm{p}, 3 \mathrm{n})$ reaction at $37 \mathrm{MeV}$. Once this cross section was obtained, we were able to work backward in order to determine the initial activity of ${ }^{246}$ Es. Using the cross section of ${ }^{247}$ Es we were able to calculate how many ${ }^{247}$ Es events would have been counted, taking the detection efficiency into account. The ${ }^{247}$ Es events were then subtracted from the area of the $7.3 \mathrm{MeV} \alpha$-peak, and a subsequent two-component halflife analysis of the revised peak area resulted in a component with a half-life of $6.0 \pm 1.2$ $\min \left({ }^{246} \mathrm{Es}\right)$ and a longer component $\left({ }^{245} \mathrm{Cf}\right.$ and ${ }^{246} \mathrm{Cf}$.) The initial $\alpha$-activity of ${ }^{246} \mathrm{Es}$ was calculated to be $654 \pm 18$ counts/min, and the cross section of the ${ }^{249} \mathrm{Cf}(\mathrm{p}, 4 \mathrm{n})$ reaction was $6 \pm 4 \mu \mathrm{b}$. 


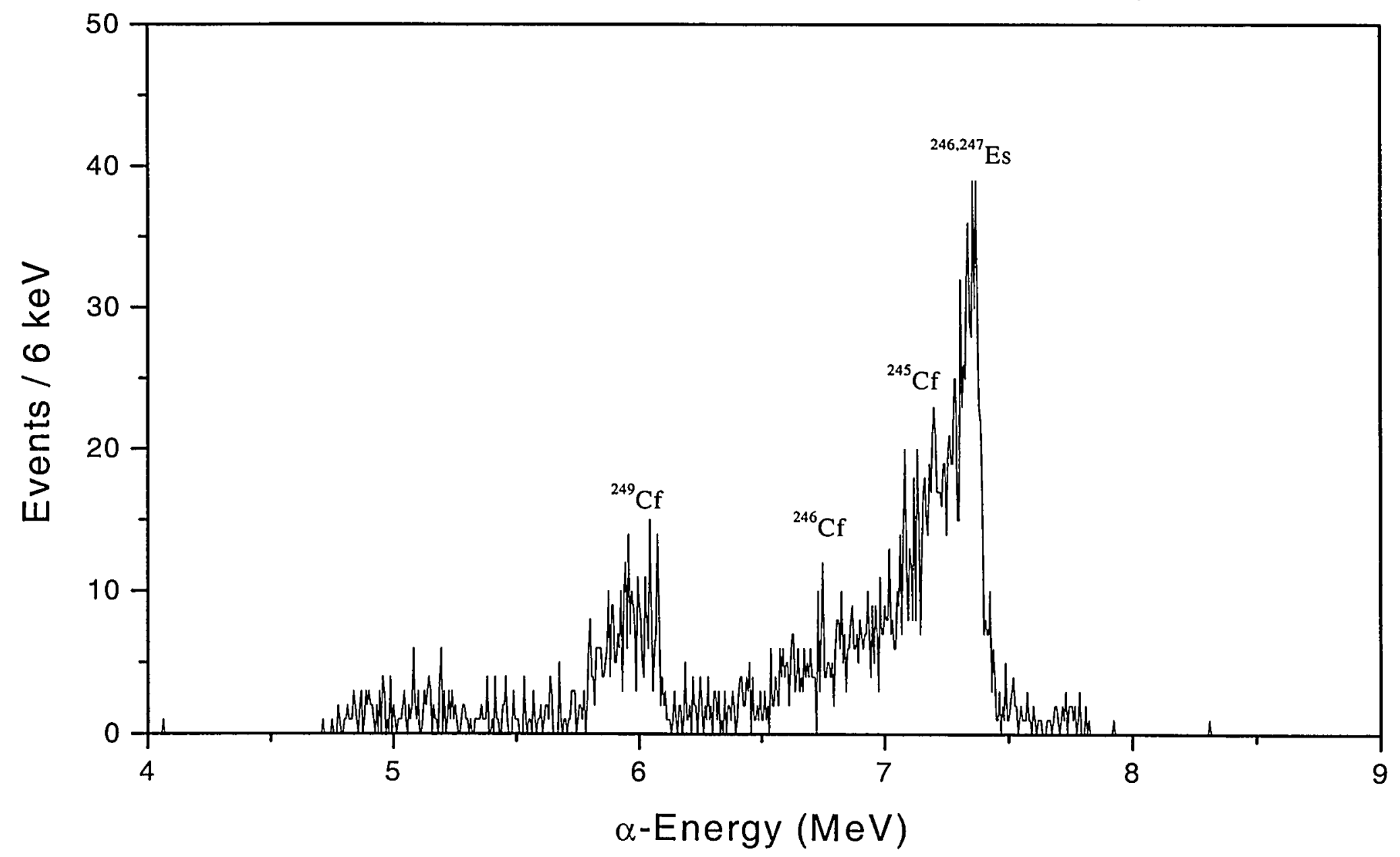

Figure 6.12: $\alpha$-spectrum from the ${ }^{249} \mathrm{Cf}(\mathrm{p}, \mathrm{xn})^{250-\mathrm{x}}$ Es reaction at $37 \mathrm{MeV}$. A 1-min stepping interval was used in the MG for a total of $80 \mathrm{~min}$ of collection and counting. This spectrum was taken from the top detector of the second detector pair. 
Figure 6.13 shows one of the $\alpha$-spectra recorded with a 2-min stepping interval at a proton energy of $18 \mathrm{MeV}$. A total of three MG wheels were recorded at this energy and stepping interval for a total of $480 \mathrm{~min}$ of collection and counting. The $\alpha$-energy resolution is even worse here because twice as much $\mathrm{KCl}$ salt was deposited on the foils than during the 1-min stepping interval. The large peak at $6.87 \mathrm{MeV}$ was integrated from 6.2 MeV to $7.0 \mathrm{MeV}$ and analyzed using MLDS with a three-component fit. The halflives of all three components were allowed to vary. The code identified half-lives of $23 \pm$ $3 \min \left({ }^{248} \mathrm{Es}\right)$ and $98 \pm 5 \min \left({ }^{249} \mathrm{Es}\right)$, and a very long component $\left({ }^{246} \mathrm{Cf}\right)$ was also resolved. In addition, the $\alpha$-peak at $7.3 \mathrm{MeV}$ was integrated from $7.2 \mathrm{MeV}$ to $7.4 \mathrm{MeV}$ and was analyzed with MLDS using a one-component fit, resulting in a half-life of $4.7 \pm$ $0.2 \min \left({ }^{247} \mathrm{Es}\right.$.) The initial activities of these nuclides were $423 \pm 15$ counts/min $\left({ }^{248} \mathrm{Es}\right)$, $138 \pm 11$ counts/min $\left({ }^{249} \mathrm{Es}\right), 396 \pm 14$ counts/min $\left({ }^{247} \mathrm{Es}\right)$ and ${ }^{246} \mathrm{Cf}$ had an initial activity that was negligible. Based on these initial activities, the corresponding production cross sections were determined to be $11 \pm 9 \mu \mathrm{b}$ for ${ }^{247}$ Es, $1300 \pm 1200 \mu \mathrm{b}$ for ${ }^{248}$ Es and $840 \pm$ $670 \mu \mathrm{b}$ for ${ }^{249}$ Es. The large errors associated with these cross sections are mostly due to large uncertainties in the yield of the LIM transport system. The efficiency of the LIM system will be discussed in section 6.2.3.1.

\subsubsection{2 $\mathrm{P}_{\mathrm{DF}}$ and $\log f t$ Values}

One coincident fission event was observed during the ${ }^{246}$ Es experiment. This was not a background event because background measurements taken before the experiment showed the coincident fission rate in each detector to be less than one fission per day. The event was assigned to the ECDF of ${ }^{246}$ Es. No other einsteinium isotopes produced in 


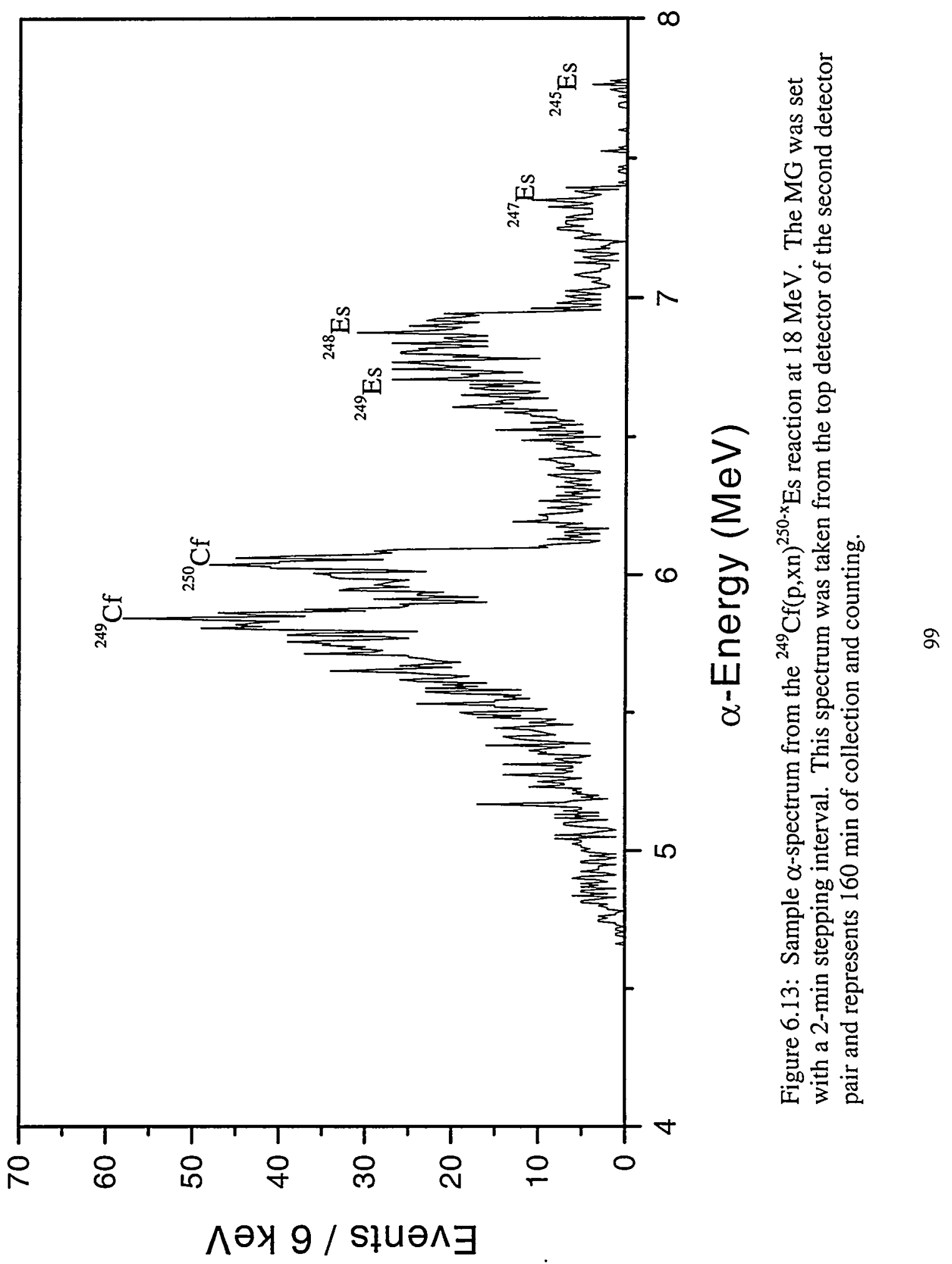


the ${ }^{249} \mathrm{Cf}(\mathrm{p}, \mathrm{xn})^{250-\mathrm{x}}$ Es reaction are known to undergo ECDF except for ${ }^{248}$ Es. However, no ${ }^{248} E s$ was observed in the $\alpha$-spectrum and it was assumed that none was produced because the beam energy was too high for the $2 \mathrm{n}$ exit channel to compete. All of the isotopes observed in the $\alpha$-spectrum were from the $3 n, 4 n$ and $5 n$ exit channels. Based on the production cross section reported by Hatsukawa et al. [HAT89] and an approximate LIM system efficiency of 50\% [HAL89], we had originally expected to see thousands of fission events per day.

Based on one fission event, an initial ${ }^{246} \mathrm{Es} \alpha$-activity of $654 \mathrm{~min}^{-1}$ and the known $\alpha-$ and EC-branches of $10 \%$ and $90 \%$, respectively [FIR96], a $\mathrm{P}_{\mathrm{DF}}$ of $(3.7 \pm 3.7) \times 10^{-5}$ was determined for ${ }^{246} \mathrm{Es}$. This agrees with the estimate of $3 \times 10^{-5}$ previously reported by Gangrskii et al. [GAN80]. Based on the relationship between $P_{D F}$ and $Q_{E C}$ shown in Figure 2.2 and a $\mathrm{Q}_{\mathrm{EC}}$ of 3.64 MeV [MÖL95], our value for the $\mathrm{P}_{\mathrm{DF}}$ of ${ }^{246} \mathrm{Es}$ seems reasonable. Using the same $\mathrm{Q}_{\mathrm{EC}}$ value and the information given by Firestone et al. [FIR96] a $\log f t$ of approximately 5.9 can be calculated for the ground state to ground state ${ }^{246}$ Es EC-transition. The predicted nuclear deformation $(\beta)$ of ${ }^{246} \mathrm{Es}$ is 0.217 [MÖL95], the same as for ${ }^{244}$ Es. Based on this deformation and the single-particle Nilsson diagrams [FIR96] the $99^{\text {th }}$ proton is most likely assigned to the $7 / 2^{+}[633]$ level. The assignment of the $147^{\text {th }}$ neutron is very dependent on deformation. At $\beta=0.2$, the neutron would be in the 7/2[743] level. Somewhere between a deformation of 0.2 and 0.23 this assignment changes to the $7 / 2^{+}[624]$ level. Since ${ }^{244}$ Es and ${ }^{246}$ Es are predicted to have equal $\beta$ values, their neutron assignments would not be the same. Therefore, the $147^{\text {th }}$ neutron is most likely $7 / 2^{+}[624]$, which can combine with the $7 / 2^{+}[633]$ proton level to form a nucleus with a ground state spin and parity assignment of $0^{+}$to $7^{+}$. The most 
likely assignment for ${ }^{246} \mathrm{Es}$ is $0^{+}$resulting in an ${ }^{246} \mathrm{Es}$ to ${ }^{246} \mathrm{Cf} \mathrm{EC}$-transition of $\Delta \mathrm{J}^{\pi}=0^{\text {no }}$. This type of transition is usually indicative of a superallowed transition with a $\log f t$ value in the range of 3.0 to 4.0 [KRA88]. However, this particular transition is isospin forbidden, which means that $\Delta \mathrm{T} \neq 0$ for the $\mathrm{EC}$-transition where $\mathrm{T}=(\mathrm{N}-\mathrm{Z}) / 2$ in the parent and daughter nuclei. Isospin forbidden transitions typically have $\log f t$ values that are greater than or equal to 6.5. The $\log f t$ of 5.9 calculated for this transition seems like it may be too low, which means that either the $\beta$ predicted for ${ }^{246}$ Es is incorrect or else this particular transition is faster than other isospin forbidden transitions in this region.

Four coincident fission events were observed during the experiment using 18$\mathrm{MeV}$ protons. These fissions were attributed to the ECDF of ${ }^{248}$ Es. Based on the small amount of ${ }^{246} \mathrm{Cf}$ seen in the spectra, too little ${ }^{246} \mathrm{Es}$ was produced at this energy to account for this many fission events. Based on the cross sections in Figure 3.1, one would not expect to produce very much ${ }^{246} \mathrm{Es}$ at $18 \mathrm{MeV}$. If these fissions were from the ECDF of ${ }^{246} \mathrm{Es}$, it would result in a $\mathrm{P}_{\mathrm{DF}}$ value for ${ }^{246} \mathrm{Es}$ that would be much higher than what was observed at $37 \mathrm{MeV}$. Therefore, the fission events are assigned to the ECDF of ${ }^{248} \mathrm{Es}$. As in the case of ${ }^{246} \mathrm{Es}$, we had originally expected approximately 2000 fission events per day, based on the cross section from Figure 3.1 and an estimated LIM efficiency of 50\% [HAL89].

Based on the four fission events, an initial ${ }^{248}$ Es $\alpha$-activity of $423 \mathrm{~min}^{-1}$ and the known $\alpha$ - and EC-branches of $0.3 \%$ and $99.7 \%$, respectively [FIR96], a $P_{D F}$ of $(3.5 \pm$ 1.8) $\times 10^{-6}$ was determined for ${ }^{248}$ Es. This is an order of magnitude larger than the estimated value of $3 \times 10^{-7}$ reported by Gangrskii et al. [GAN80]. Based on the relationship between $\mathrm{P}_{\mathrm{DF}}$ and $\mathrm{Q}_{\mathrm{EC}}$ shown in Figure 2.2 and a $\mathrm{Q}_{\mathrm{EC}}$ of 3.05 MeV [MÖL95], 
this new value for the $P_{D F}$ of ${ }^{248}$ Es seems very reasonable. The line in Figure 2.2 is not a best fit to the data and is meant only to guide the eye. An actual fit to the data could result in a line with a much different slope, allowing our $P_{D F}$ value for ${ }^{248} E s$ to fit the apparent trend of $\mathrm{P}_{\mathrm{DF}}$ values observed in the figure (see section 7.5 for a discussion about a fit to the $P_{D F}$ data.)

Based on a $\mathrm{Q}_{\mathrm{EC}}$ of $3.05 \mathrm{MeV}$ and using the information in Firestone et al. [FIR96], a $\log f t$ value of 6.1 for the ground state to ground state ${ }^{248}$ Es EC-transition can be calculated. As in the case of ${ }^{244}$ Es and ${ }^{246} E s$, the predicted $\beta$ for ${ }^{248}$ Es is 0.217 [MÖL95]. Using the single-particle Nilsson diagrams [FIR96], the $99^{\text {th }}$ proton at this deformation is most likely assigned to the $7 / 2^{+}[633]$ level. The neutron levels in these neutron deficient einsteinium isotopes have been very dependent on deformation, and the assignments can change completely with only a small change in nuclear deformation. At a deformation of 0.2 the $149^{\text {th }}$ neutron in ${ }^{248}$ Es would be assigned to the $7 / 2^{+}[624]$ level. Between deformations of 0.2 and 0.23 the level assignment changes to 9/2-[734]. Based on the predicted value of $\beta, 9 / 2[734]$ is the most likely assignment, and follows from the assignments previously made for ${ }^{244} \mathrm{Es}$ and ${ }^{246} \mathrm{Es}$. If the $149^{\text {th }}$ neutron is assigned to the $9 / 2[734]$ level, when combined with the $7 / 2^{+}[633]$ proton level, the ${ }^{248}$ Es nucleus would have a ground state spin and parity in the range of $1^{-}$to $8^{-}$. The most likely assignment for ${ }^{248}$ Es is $1^{-}$, which means that the resulting ground state EC-transition would be $\Delta \mathrm{J}^{\pi}=1^{\text {yes }}$. A change in momentum of one unit coupled with a parity change is indicative of a first-forbidden decay. A $\log f t$ of 6.1 is appropriate for a first-forbidden transition in the actinides, indicating that the $149^{\text {th }}$ neutron should be assigned to the $9 / 2^{-}$ [734] neutron level and $\beta$ is greater than 0.2 as predicted. 


\subsubsection{X-ray/Fission Coincidence Measurements}

The experimental procedure for studying $\mathrm{x}$-rays in coincidence with fission fragments was described in detail in section 5.2. Each chemically separated sample counted in the coincidence setup while the next MG wheel was being measured. Figure 6.14 shows the $x$-ray and $\gamma$-ray spectrum from one of the $x$-ray detectors in the setup, along with the $\alpha$-spectrum from the Si detector. Immediately, one notices the very poor energy resolution of the $\alpha$-detector. Because of the setup used, this detector was operated in air, which attributed to the terrible resolution seen in Figure 6.14. The enormous ${ }^{249} \mathrm{Cf}$ peak was due to target material that was knocked out by the beam. At higher beam energies more atoms are knocked out of the target, so at $37 \mathrm{MeV}$, the amount of ${ }^{249} \mathrm{Cf}$ in the spectrum is huge. There was so much ${ }^{249} \mathrm{Cf}$ present that it swamped everything else in the spectrum making it impossible to determine what other isotopes were present. A total of 40 fission events were recorded at $37 \mathrm{MeV}$ from 17 samples, but a $P_{D F}$ could not be calculated from these data because it was impossible to determine how much ${ }^{246} \mathrm{Es}$ was present underneath the large ${ }^{249} \mathrm{Cf}$ peak. Looking at the $\mathrm{x}$-ray spectrum in Figure 6.14 , one sees all of the characteristic $\mathrm{x}$-rays and $\gamma$-rays from the $\alpha$-decay of ${ }^{249} \mathrm{Cf}$. The most prominent $\mathrm{x}$-rays associated with the EC-decay of einsteinium are $115 \mathrm{keV}\left(\mathrm{K}_{\alpha 1}\right)$, $109 \mathrm{keV}\left(\mathrm{K}_{\alpha 2}\right)$ and $130 \mathrm{keV}\left(\mathrm{K}_{\beta 1}\right)$. Because the $\mathrm{Cm} \mathrm{K}_{\alpha 1} \mathrm{x}$-ray is also at $109 \mathrm{keV}$, it is impossible to differentiate between the californium and curium $\mathrm{x}$-rays in this peak. However, neither the $115-\mathrm{keV}$ nor the $130-\mathrm{keV} \mathrm{x}$-rays were observed in the spectrum. Any $\mathrm{x}$-rays originating from the EC-decay of einsteinium were buried beneath the ${ }^{249} \mathrm{Cf}$ background. It was obvious that the amount of einsteinium produced during the cyclotron bombardments was much lower than we had expected based on the cross 


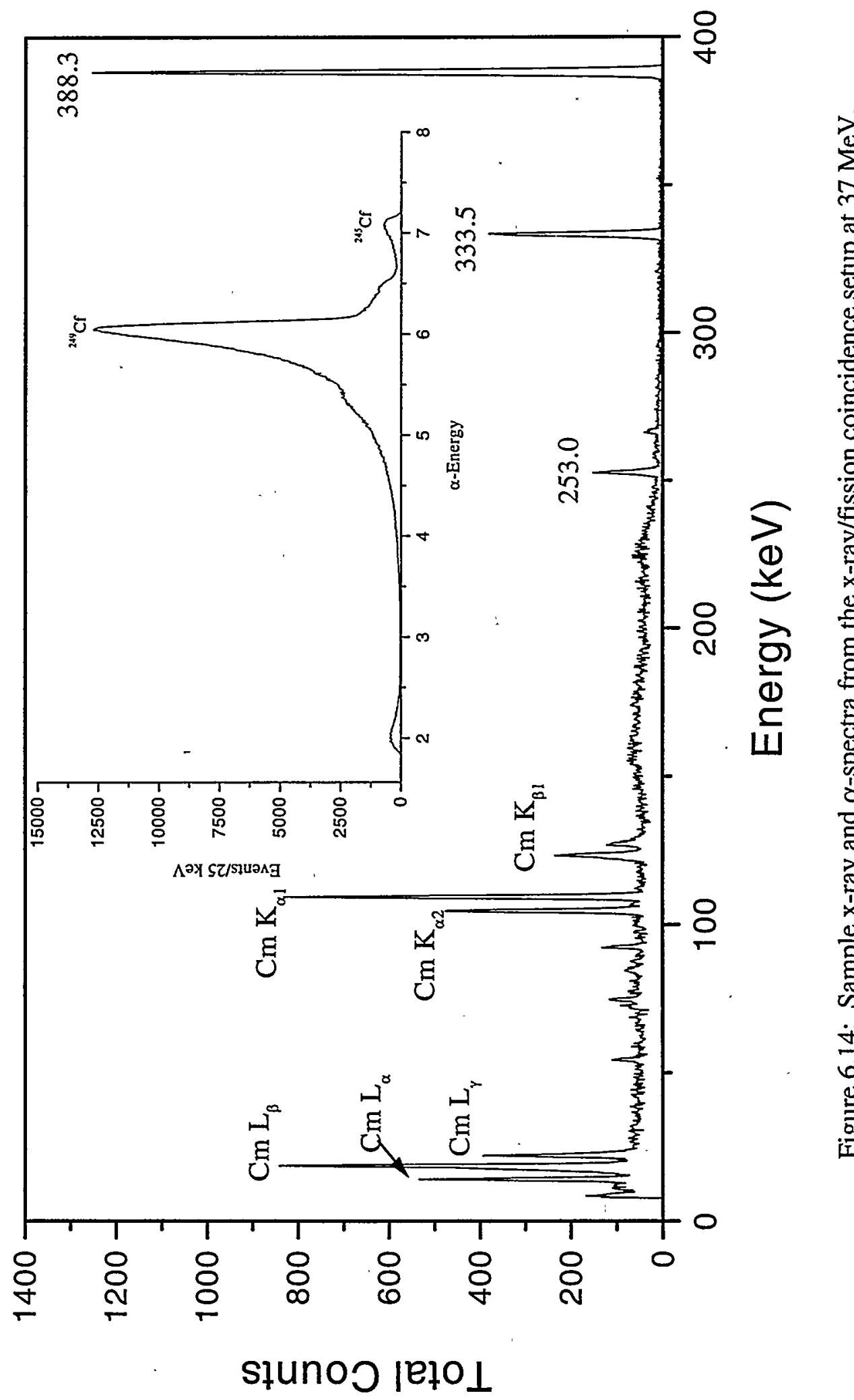


sections reported by Hatsukawa et al. [HAT89]. This was confirmed by the low number of fission events observed during the on-line measurements and by the absence of any $\mathrm{x}$ rays associated with the EC-decay of einsteinium.

The coincidence data recorded at $18 \mathrm{MeV}$ was not much different. The $\mathrm{x}$-ray spectra showed no x-rays of the correct energy for einsteinium EC-decay, only those associated with the $\alpha$-decay of ${ }^{249} \mathrm{Cf}$. At this lower energy, there was definitely less ${ }^{249} \mathrm{Cf}$ in the $\alpha$-spectra, but it still overwhelmed the much smaller ${ }^{248}$ Es and ${ }^{249}$ Es peaks. Two fission events were recorded from a total of five samples. The initial activity of ${ }^{248} \mathrm{Es}$ could not be determined because there was too much interference from the ${ }^{249} \mathrm{Cf}$ background. Therefore, a $\mathrm{P}_{\mathrm{DF}}$ could not be determined from the coincidence data. Einsteinium and californium both exhibit a $3+$ oxidation state, which means that both elements were extracted into the TTA phase during the chemical separation. To remove this large californium background, it would be necessary to perform a chemical separation that separates $3+$ actinides from each other, thus creating a pure einsteinium fraction for counting. This was not originally done because of the limited amount of cyclotron time allotted for this particular experiment.

As mentioned previously, the fission rates observed during these experiments were much lower than originally predicted. Also, no x-rays from einsteinium EC-decay were observed in the $\mathrm{x}$-ray spectra. Using the initial activities of ${ }^{246} \mathrm{Es}$ and ${ }^{248} \mathrm{Es}$ given in section 6.1.3.1, estimates of the production cross sections for ${ }^{246} \mathrm{Es}$ and ${ }^{248} \mathrm{Es}$ were originally determined using an assumed LIM system efficiency of 50\%. The cross section for ${ }^{246}$ Es agreed relatively well with the value reported by Hatsukawa et al. [HAT89]. However, the original cross section of ${ }^{248}$ Es (not the value quoted in section 
6.1.3.1) was more than an order of magnitude lower than the previously reported values. All of the other experimental parameters were known, except for the absolute gas-jet transport efficiency. Therefore, it was theorized that perhaps the efficiency of the LIM gas-jet transport system was much lower than the $50 \%$ we had estimated, at least in certain instances. Hall [HAL89] had measured LIM efficiencies of 50\% to $95 \%$ in previous experiments so $50 \%$ was used in the cross section determinations for ${ }^{246}$ Es and ${ }^{248} \mathrm{Es}$ as a conservative estimate. It was then decided that the absolute efficiency of the LIM system should be measured for future cross section determinations. A low LIM transport efficiency would explain why the fission and $\alpha$-particle rates were so much lower during the experiments than originally predicted. The results of this yield determination are discussed in section 6.2.3.1.

\subsection{Einsteinium Excitation Functions}

\subsection{1 ${ }^{233} \mathrm{U}\left({ }^{14} \mathrm{~N}, \mathrm{xn}\right){ }^{247-\mathrm{x}}$ Es Reactions}

An experiment to determine the excitation function for ${ }^{14} \mathrm{~N}\left({ }^{233} \mathrm{U}, \mathrm{xn}\right)^{247-\mathrm{x}} \mathrm{Es}$ reactions using a single ${ }^{233} \mathrm{U}$ target was performed at beam energies of $80 \mathrm{MeV}, 87 \mathrm{MeV}$, $93 \mathrm{MeV}$ and $100 \mathrm{MeV}$ (see section 4.3 for more information.) The energy loss of the projectiles as they travel through the ${ }^{233} \mathrm{U}$ target material is approximately $0.5 \mathrm{MeV}$ at each beam energy [NOR70]. The experimental procedure is described in section 5.3.1. Figure 6.4 (section 6.1.1.2) shows a typical $\alpha$-spectrum from the ${ }^{233} U\left({ }^{14} \mathrm{~N}, \mathrm{xn}\right)^{247-\mathrm{x}} \mathrm{Es}$ reaction at $87 \mathrm{MeV}$. The observed $\alpha$-spectra did not change much as a function of ${ }^{14} \mathrm{~N}$. projectile energy. 
As discussed in section 6.1.1.1, any fission events observed from this reaction could only come from the ECDF of ${ }^{242}$ Es. Therefore, the number of coincident fission pairs observed at each energy served as an indication of the production of ${ }^{242}$ Es. Background measurements taken before the experiment showed less than one coincident fission event per day per detector could be attributed to background events.

The fission results presented in Table 6.3 match the predictions of the SPIT code [SIK67] (Figure 4.5) where one would expect a lower number of ${ }^{242}$ Es nuclei produced at $80 \mathrm{MeV}$ than at $87 \mathrm{MeV}$, and therefore fewer fission events. Likewise, at $93 \mathrm{MeV}$ there should be fewer events than at $87 \mathrm{MeV}$, and $100 \mathrm{MeV}$ should produce even fewer. Based on the fission results alone, it appeared that SPIT correctly predicted the shape of the excitation function at higher projectile energies. However, SPIT predicts that the excitation function decreases dramatically at lower energies whereas the fission results imply that the low energy part of the function may decrease more gradually. SPIT predicted the peak of the excitation function at $85 \mathrm{MeV}$, and this may be close to the actual peak energy.

Table 6.3. Observed coincident fission event rates for each beam for ${ }^{233} U\left({ }^{14} N, x n\right){ }^{247-x} E s$ reactions. See section 4.3 for more information on the ${ }^{14} \mathrm{~N}$ beams.

\begin{tabular}{|ccc|}
\hline $\begin{array}{c}{ }^{14} \mathrm{~N} \text { Energy } \\
(\mathrm{MeV})\end{array}$ & $\begin{array}{c}\text { Total Measurement Time } \\
(\mathrm{h})\end{array}$ & $\begin{array}{c}\text { Coincident Fission Event Rate } \\
(\text { fissions/h) }\end{array}$ \\
\hline 80 & 9 & 0.3 \\
87 & 6 & 0.7 \\
93 & 6 & 0.2 \\
100 & 4 & 0 \\
\hline
\end{tabular}

To calculate production cross sections, the initial activity of the nuclide of interest after bombardment is determined from a half-life analysis of the nuclide using the MLDS code. Once this activity is determined, corrections must be made to the simple cross 
section equation presented in Chapter 1 (equation 1.3.) The $\alpha$-branch of the nuclide must be taken into account, as well as the yield of the gas-jet transport system and the detection efficiency. A correction must also be made for the time it takes for reaction products to travel from the target chamber to the collection site, and for the decay of the nuclide during collection. Including all of these corrections in equation 1.3 results in the final equation used for calculating cross sections from the $\alpha$-data obtained during the excitation function experiments.

$$
\sigma=\frac{C_{o}}{\text { branch } \cdot \text { yield } \cdot \varepsilon \cdot I \cdot N \cdot e^{-\lambda \cdot t_{\text {trans }}} \cdot\left(1-\mathrm{e}^{-\lambda \cdot t_{\text {irr }}}\right) \cdot N s}
$$

$\mathrm{C}_{\mathrm{o}}$ is the initial activity of the nuclide after collection as determined by MLDS (counts/time), branch is the decay branch for the type of decay under consideration, yield is the efficiency of the gas-jet transport system, $\varepsilon$ is the overall detection efficiency, $\mathrm{I}$ is the number of incident beam particles per unit time $(\mathrm{p} \mu \mathrm{A}), \mathrm{N}$ is the number of target nuclei per unit area $\left(\mathrm{cm}^{-2}\right), \lambda$ is the decay constant of the nuclide $\left(\right.$ time $\left.{ }^{-1}\right), \mathrm{t}_{\text {trans }}$ is the time it takes for reaction products to travel from the target chamber to the collection site, $t_{\text {irr }}$ is the irradiation interval for a single sample and $\mathrm{Ns}$ is the number of samples (foils) measured. The term $\varepsilon$ not only takes the efficiency of the detector into account, but also the intensity of the $\alpha$-group that was integrated to find the initial activity of the nuclide. In the $\alpha$-spectra recorded at $80 \mathrm{MeV}$ and $87 \mathrm{MeV}$ there was a distinct $\alpha$-peak at 7.9 $\mathrm{MeV}$ corresponding to the $\alpha$-decay of ${ }^{243}$ Es. An energy region incorporating both ${ }^{243} \mathrm{Es} \alpha$-particles from $7.80 \mathrm{MeV}$ to $7.95 \mathrm{MeV}$ was integrated, and a half-life analysis of the $\alpha$-peak was performed for both energies using MLDS. At $80 \mathrm{MeV}$ a one-component fit resulted in a half-life of $23 \pm 2 \mathrm{~s}$, very close to the reported 21 -s half-life of ${ }^{243} \mathrm{Es}$ 
[FIR96]. A two-component fit was also used to try to distinguish ${ }^{242}$ Es from ${ }^{243}$ Es in the 7.9 $\mathrm{MeV} \alpha$-peak. However, the code could only identify a single component with a halflife of $23 \mathrm{~s}$, even when both half-lives were held constant by the program. Either the code could not distinguish between the two similar half-lives or ${ }^{243}$ Es was the only component in the $\alpha$-peak. The latter is probably true, based on the small $\alpha$-branch of ${ }^{242}$ Es calculated in section 6.1 .1 .2 . At $87 \mathrm{MeV}$ a one-component fit to the $\alpha$-peak resulted in a half-life of $36 \pm 3 \mathrm{~s}$, too long to just be ${ }^{243} \mathrm{Es}$. Two and three-component fits of the peak were also attempted, and there were ultimately two components in the $\alpha$ peak, one with a $23 \pm 3$-s half-life and the other having a half-life closer to $1 \mathrm{~min}$. The shorter-lived activity is ${ }^{243} \mathrm{Es}$, and the longer-lived activity is most likely ${ }^{218} \mathrm{Fr}$ with an $\alpha$ decay energy of $7.867 \mathrm{MeV}$ [FIR96]. Because ${ }^{218} \mathrm{Fr}$ is so short lived $\left(\mathrm{t}_{1 / 2}=1 \mathrm{~ms}\right.$ [FIR96]), it appears to decay with the characteristic half-life of its parent, ${ }^{222} \mathrm{Ac}^{m}\left(\mathrm{t}_{1 / 2}=63 \mathrm{~s}\right.$ [FIR96]). Several actinium isotopes are formed in the interaction between the ${ }^{14} \mathrm{~N}$ beam and lead impurities in the target. Again, there was no evidence of $11-\mathrm{s}{ }^{242}$ Es in the $\alpha$ peak, which was probably due to its small $\alpha$-branch. Figures 6.15 and 6.16 show the decay curves of the 7.9-MeV $\alpha$-peak at projectile energies of $80 \mathrm{MeV}$ and $87 \mathrm{MeV}$, respectively.

The initial activity of ${ }^{243} E$ s at the end of collection was $6.5 \pm 0.5$ counts/s and 5.3 \pm 0.6 counts/s at projectile energies of $80 \mathrm{MeV}$ and $87 \mathrm{MeV}$, respectively. Using equation 6.1 , cross sections of $33 \pm 17 \mathrm{nb}$ at $80 \mathrm{MeV}$ and $48 \pm 25 \mathrm{nb}$ at $87 \mathrm{MeV}$ were calculated for the ${ }^{233} \mathrm{U}\left({ }^{14} \mathrm{~N}, 4 \mathrm{n}\right)$ reaction. The large errors are mostly due to the relative uncertainty of the yield of the gas-jet system (yield $=60 \pm 20 \%$ [WIL97].) The SPIT predictions [SIK67] for these energies are $70 \mathrm{nb}$ at $80 \mathrm{MeV}$ and $20 \mathrm{nb}$ at $87 \mathrm{MeV}$. 


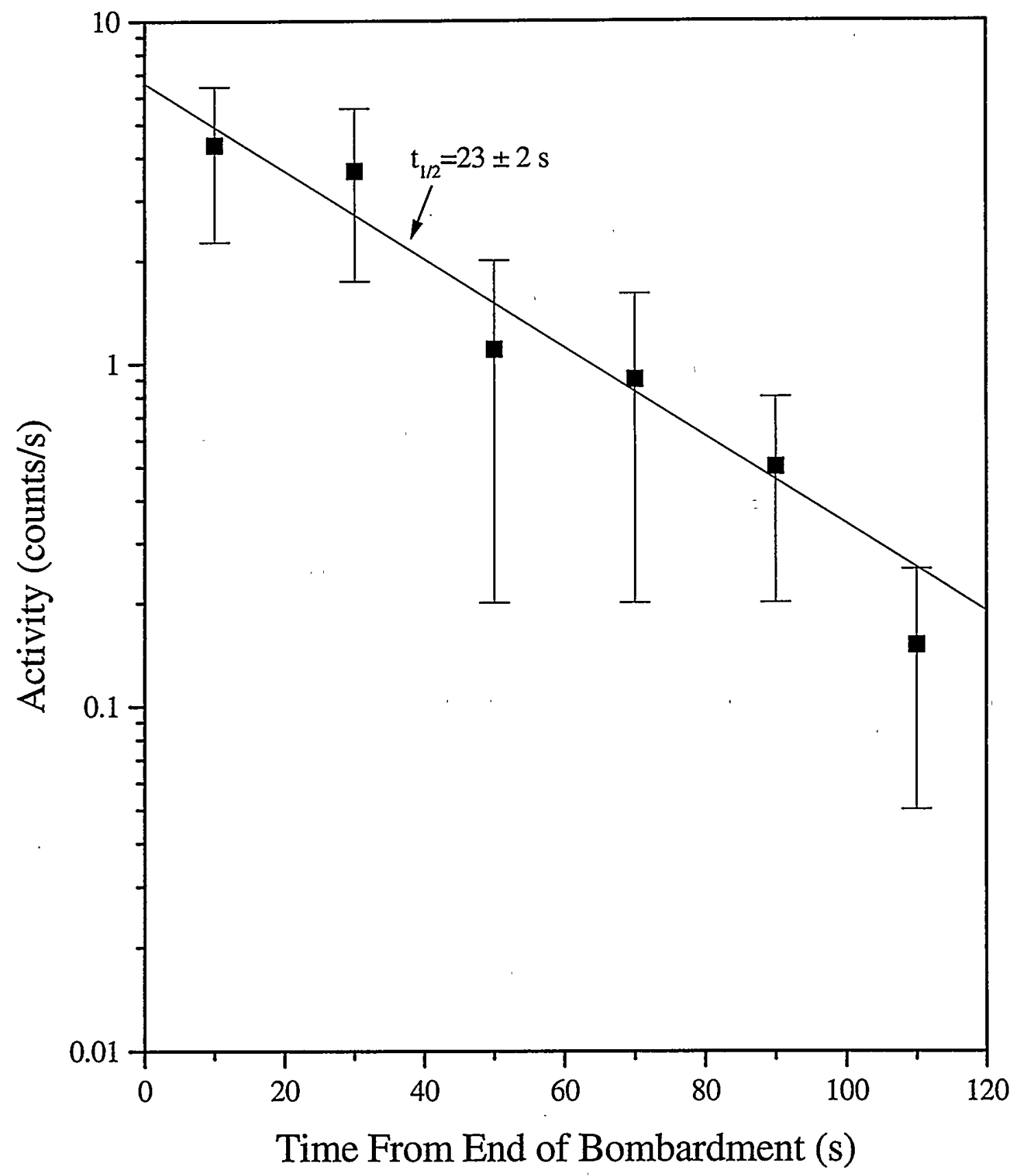

Figure 6.15: Decay curve of 7.9-MeV $\alpha$-peak integrated from 7.80 MeV to $7.95 \mathrm{MeV}$ from the decay of ${ }^{243} \mathrm{Es}$ at a projectile energy of $80 \mathrm{MeV}$. The line represents the best fit to the data using the MLDS code. The data were collected and counted for a total of $9 \mathrm{~h}$. 


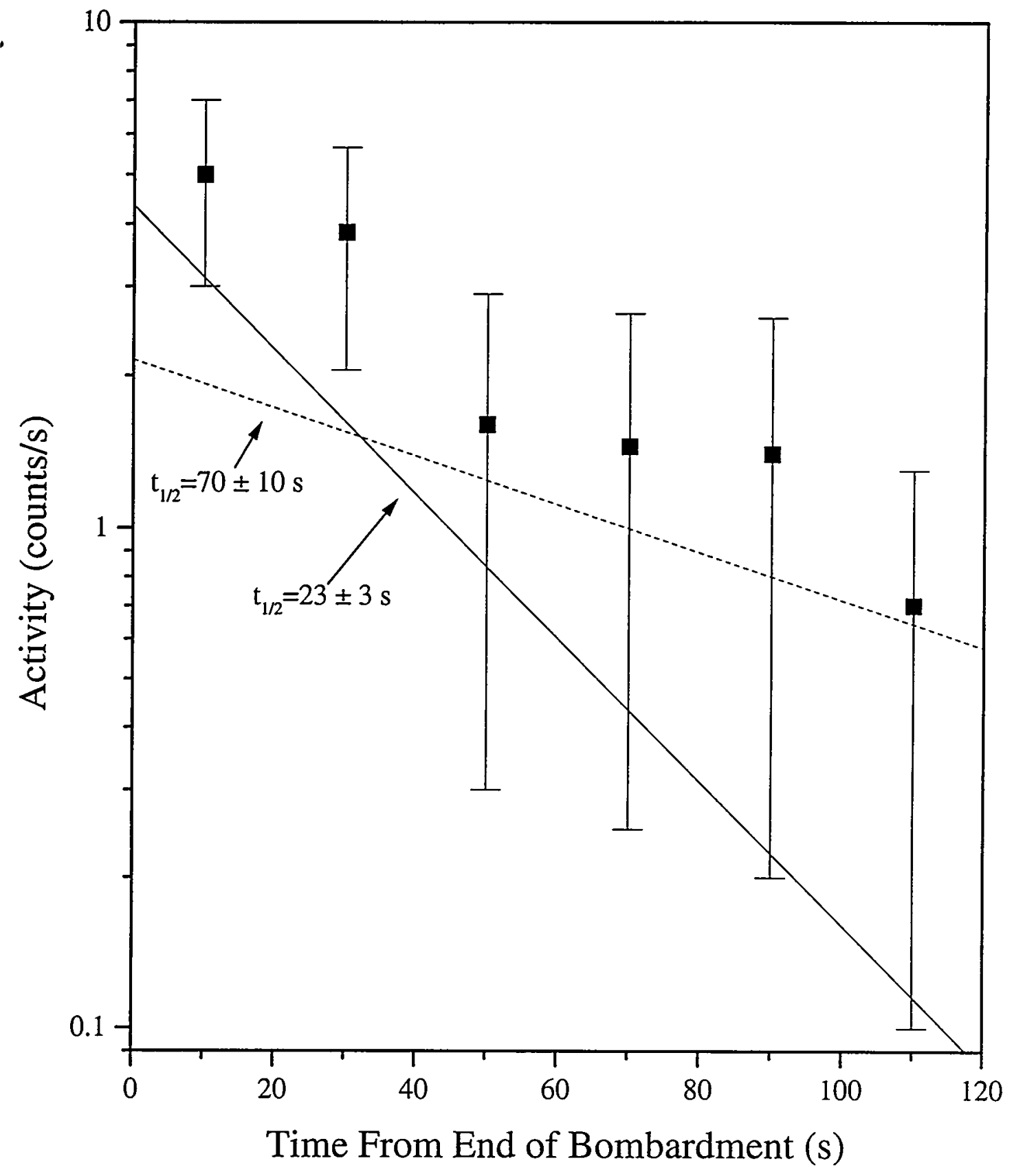

Figure 6.16: Decay curve of 7.9-MeV $\alpha$-peak integrated from 7.80 MeV to $7.95 \mathrm{MeV}$ at a projectile energy of $87 \mathrm{MeV}$. The lines represent a two-component fit to the data using the MLDS code. The shorter component is ${ }^{243} \mathrm{Es}$ and the longer component is from interfering activities. The data were collected and counted for a total of $6 \mathrm{~h}$. 
At both $80 \mathrm{MeV}$ and $87 \mathrm{MeV}$, the SPIT code was less than a factor of three lower than the actual cross section, but it appeared that SPIT might have incorrectly predicted the peak energy of the excitation function. SPIT gave a larger production cross section for $80 \mathrm{MeV}$ than for $87 \mathrm{MeV}$, and the experimental data illustrate the opposite. Also, SPIT predicted a very steep function for ${ }^{243} \mathrm{Es}$ (see Figure 4.5 ) with a $50 \mathrm{nb}$ difference between the values at $80 \mathrm{MeV}$ and $87 \mathrm{MeV}$. The experimental values at these energies are only 15 $\mathrm{nb}$ apart, indicating that the low energy part of the function may not rise so sharply, but instead may increase more gradually with energy. Both the fission and $\alpha$-results imply that SPIT can predict the relative magnitudes of cross sections and approximate peak energies in this region. To determine whether or not the shape of the function at low energies is predicted correctly, additional measurements using lower energy projectiles would have to be made.

At projectile energies of $93 \mathrm{MeV}$ and $100 \mathrm{MeV},{ }^{243} \mathrm{Es}$ could not be identified in the decay analysis of the $7.9 \mathrm{MeV} \alpha$-peaks. At both energies the only component identified in the peak was ${ }^{218} \mathrm{Fr}$. From the decay analysis, upper limits of $20 \mathrm{nb}$ and $15 \mathrm{nb}$ were measured for the production cross sections of ${ }^{243} \mathrm{Es}$ at $93 \mathrm{MeV}$ and $100 \mathrm{MeV}$, respectively. Figure 6.17 shows the experimentally determined cross sections for ${ }^{243} \mathrm{Es}$ along with the SPIT prediction [SIK67] for the ${ }^{233} \mathrm{U}\left({ }^{14} \mathrm{~N}, 4 \mathrm{n}\right){ }^{243}$ Es reaction.

Both the fission and $\alpha$-data showed a similar trend of einsteinium production for ${ }^{242}$ Es and ${ }^{243}$ Es. The order of production from most to least was $87 \mathrm{MeV}, 80 \mathrm{MeV}, 93$ $\mathrm{MeV}$ and $100 \mathrm{MeV}$. Based on these results, it was decided to use $87-\mathrm{MeV}^{14} \mathrm{~N}$ projectiles for the third and final ${ }^{242}$ Es ECDF experiment (see section 6.1.1.2) to maximize production of ${ }^{242}$ Es and the number of fission events detected during the experiment. 


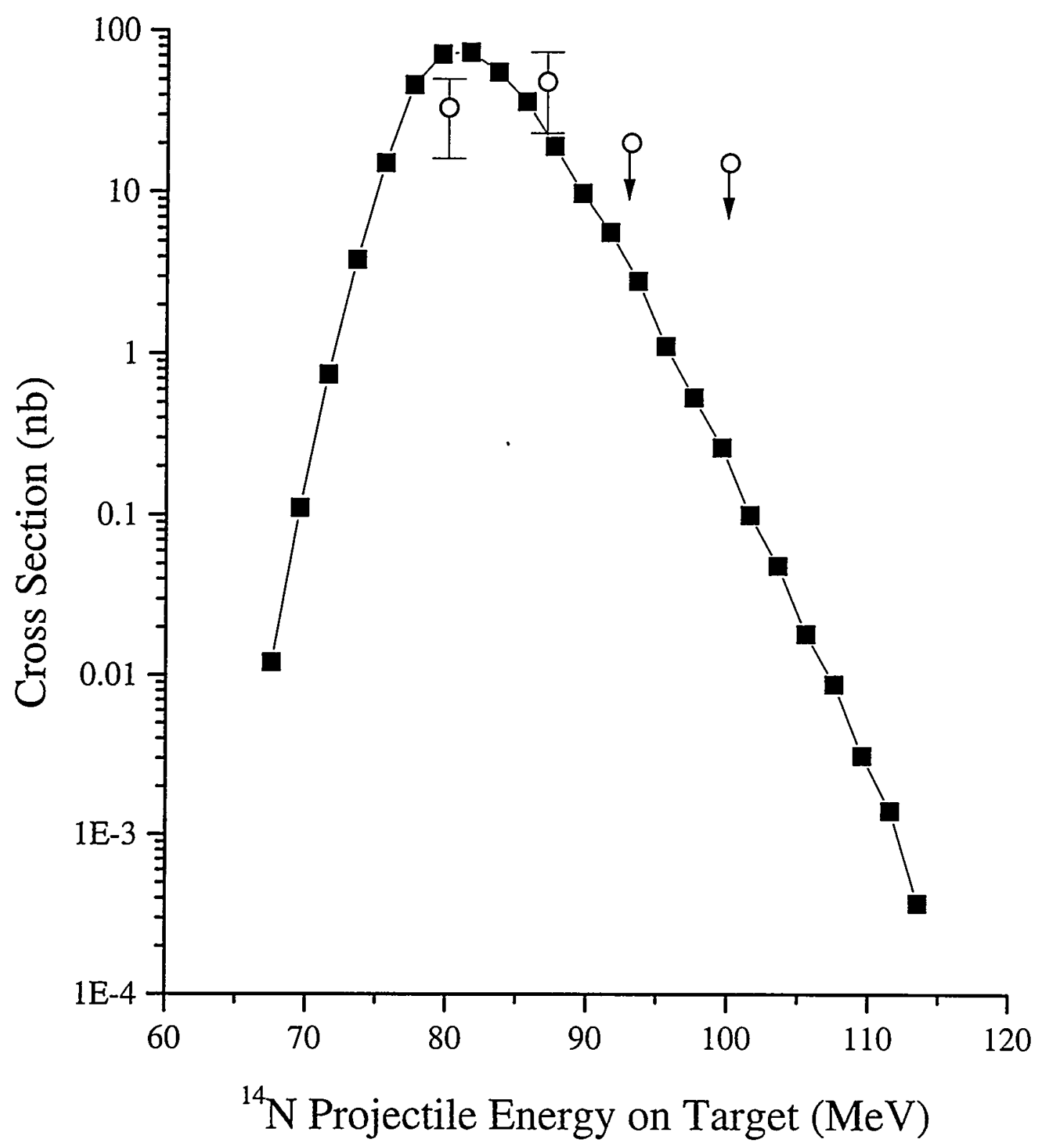

Figure 6.17: Experimentally determined cross sections for the ${ }^{233} \mathrm{U}\left({ }^{14} \mathrm{~N}, 4 \mathrm{n}\right){ }^{243}$ Es reaction (open circles) and the SPIT prediction (closed squares [SIK67]) for the same reaction. The experimental points at $93 \mathrm{MeV}$ and $100 \mathrm{MeV}$ represent upper limits only. 


\subsection{2 ${ }^{233} \mathrm{U}\left({ }^{15} \mathrm{~N}, \mathrm{xn}\right){ }^{248-\mathrm{x}}$ Es Reactions}

The excitation function for ${ }^{233} \mathrm{U}\left({ }^{15} \mathrm{~N}, \mathrm{xn}\right){ }^{248-\mathrm{x}} \mathrm{Es}$ reactions using a single ${ }^{233} \mathrm{U}$ target was studied at projectile energies of $80 \mathrm{MeV}, 85 \mathrm{MeV}, 93 \mathrm{MeV}$ and $100 \mathrm{MeV}$ to determine the ideal beam energy for the production of ${ }^{244}$ Es via the 4 n exit channel (see section 4.3). The experimental procedure is given in section 5.3.1. The energy loss of the beam through the ${ }^{233} \mathrm{U}$ target material was approximately $0.5 \mathrm{MeV}$. Reaction products were collected and counted for a total of $5 \mathrm{~h}$ at each energy.

At $80 \mathrm{MeV}$ there was a prominent $\alpha$-peak at $7.7 \mathrm{MeV}$ observed in the spectra. This appeared to be ${ }^{245}$ Es produced via the $3 \mathrm{n}$ exit channel. The area between $7.6 \mathrm{MeV}$ and $7.8 \mathrm{MeV}$ was integrated to incorporate the strongest $\alpha$-groups of ${ }^{245}$ Es. A half-life analysis of the peak using MLDS resulted in a single component with a half-life of $1.0 \pm$ 0.2 min, confirming the identity of the nuclide as ${ }^{245}$ Es. Figure 6.18 shows the $\alpha$ spectrum recorded at $80 \mathrm{MeV}$ using 1-min steps in the $\mathrm{MG}$, and represents $80 \mathrm{~min}$ of collection and measurement.

An initial activity of $65 \pm 6$ counts/min at the end of collection was determined from the half-life analysis for ${ }^{245}$ Es. Using equation 6.1 a production cross section of 60 $\pm 23 \mathrm{nb}$ was calculated for the ${ }^{233} \mathrm{U}\left({ }^{15} \mathrm{~N}, 3 \mathrm{n}\right)$ reaction at $80 \mathrm{MeV}$. The SPIT prediction for this energy (see Figure 4.6) is approximately $250 \mathrm{nb}$, about a factor of four larger than the experimental value. The ${ }^{245}$ Es $\alpha$-peak could not be identified at higher beam energies because the amount of interfering activities produced in the reaction increased so as to completely swamp the 7.7-MeV region of the spectra.

No fission events were observed during the experiment. As discussed in section 6.1.2, based on these results, SPIT predictions, and spectra given by Hatsukawa et al. 


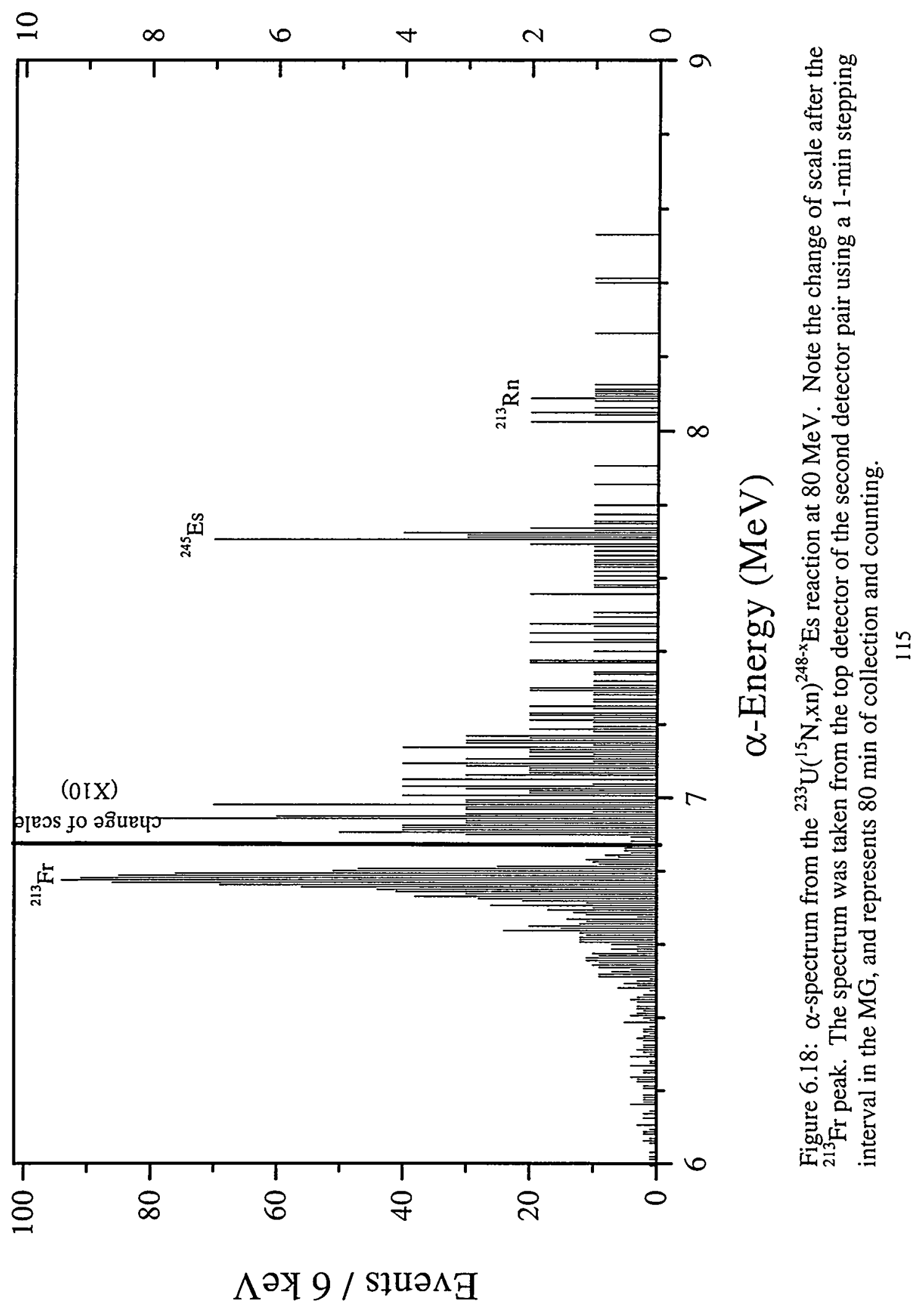


[HAT89], the ${ }^{237} \mathrm{~Np}\left({ }^{12} \mathrm{C}, 5 \mathrm{n}\right){ }^{244}$ Es reaction was ultimately chosen for the study of ECDF in ${ }^{244}$ Es. This reaction was much better for producing ${ }^{244} \mathrm{Es}$ in relatively large amounts and yielded the results presented in section 6.1.2.2.

\subsection{3 ${ }^{249} \mathrm{Cf}(\mathrm{p}, \mathrm{xn}){ }^{250-\mathrm{x}}$ Es Reaction}

\subsubsection{Efficiency of the LIM/Gas-Jet Transport System}

\subsection{Target Tests of the LIM System}

As described in section 6.1.3, the measured fission and $\alpha$-rates in the study of ECDF in ${ }^{246}$ Es and ${ }^{248}$ Es were much lower than originally expected based on the cross sections reported by Hatsukawa et al. [HAT89]. Production cross sections for ${ }^{249} \mathrm{Cf}(\mathrm{p}, \mathrm{xn}){ }^{250-\mathrm{x}}$ Es reactions were measured for ${ }^{246} \mathrm{Es}$ at $37 \mathrm{MeV}$ and ${ }^{248} \mathrm{Es}$ at $18 \mathrm{MeV}$ using an estimated yield of the LIM gas-jet transport system of $50 \%$. The result for ${ }^{248} \mathrm{Es}$ was about five times lower than that reported by Hatsukawa et al. [HAT89] and indicated that the yield of the LIM system might be lower than $50 \%$. The result for ${ }^{246}$ Es using a $50 \%$ yield, however, was actually in good agreement with the reported value (extrapolated from a cross section measured at $33 \mathrm{MeV}$ [HAT89].) Based on these observations, we decided to test the yield of the LIM system and if possible, measure an absolute efficiency for the transport of reaction products from the LIM via the $\mathrm{KCl} / \mathrm{He}$ gas-jet. We also wanted to determine why the results for ${ }^{246} \mathrm{Es}$ and ${ }^{248} \mathrm{Es}$ indicated such different LIM efficiencies.

Hall had originally reported [HAL89] that the number of delayed fission events detected during ECDF experiments increased by the number of targets used in the LIM system. In other words, 10 targets would result in 10 times more fission events than a 
single target. Our first test of the LIM system was designed to verify this increased production and to make sure that all of the targets were in fact contributing to the overall yield of reaction products. Eleven ${ }^{233} \mathrm{U}$ targets were placed in the LIM chamber in every other target slot. These targets were bombarded with $50-\mathrm{MeV}$ protons $(2.5 \mathrm{e} \mu \mathrm{a})$ to produce ${ }^{229} \mathrm{~Np}$ in a $5 \mathrm{n}$ reaction. First, the target closest to the point where the beam enters the LIM chamber was in the direction of the beam so recoiling ${ }^{229} \mathrm{~Np}$ nuclei would enter the gas stream while the other 10 targets were turned backward so the ${ }^{229} \mathrm{~Np}$ would recoil into the Be target backing. Second, the last target in the chamber was turned so it was facing the correct direction and all of the other targets were turned backward. Third, all 11 targets were turned so that they were facing correctly, and finally, the last target in the chamber was left facing the downstream direction and the other 10 targets were removed from the chamber. With each configuration, the $\alpha$-rate was recorded and compared to the other configurations.

The most interesting results arose from the third and final target configurations. With only the last target present in the chamber, an $\alpha$-rate of approximately 1 count/s was observed. When all 11 targets were in the chamber, the $\alpha$-rate only rose to approximately 6 counts/s. The $\alpha$-rate only increased by a factor of six, not the factor of 11 we expected based on the number of targets. More than one target was indeed contributing to the overall yield of reaction products transported from the LIM system, but it appeared that not all of the targets were contributing to the overall $\alpha$-rate. One possible explanation for this is perhaps some of the $\mathrm{KCl}$ aerosols are depositing on the target holders and therefore can not collect reaction products for transport. Another possible explanation is the fact that light projectiles impart very low momentum to the compound nucleus, resulting in 
very short recoil ranges of the compound nucleus (see section 4.1.2). At a proton energy of $50 \mathrm{MeV}$, the recoil range of a ${ }^{234} \mathrm{~Np}$ compound nucleus in He would only be a few tenths of a mm [NOR70]. With such a small recoil range, it is possible that thermal motion is bringing some of the reaction products back into contact with the targets before they can attach themselves to $\mathrm{KCl}$ aerosols, effectively reducing the yield of the gas-jet system [GRE98]. Whatever the reason, the target tests proved that not all of the reaction products produced from the LIM system were being collected, which could explain the low $\alpha$ - and fission rates during the ECDF experiments.

\subsection{Efficiency Measurement of the LIM System}

To measure the efficiency of the LIM system, a gold catcher foil was used to collect reaction products produced from a single ${ }^{249} \mathrm{Cf}$ in the LIM system. See section 5.3.2 for the details of the experimental procedure for the catcher foil. The proton beam was $21 \mathrm{MeV}$. A gold foil and a molybdenum foil were placed one at a time behind one ${ }^{249} \mathrm{Cf}$ target in the LIM system, which was under vacuum. The foil in each case has a collection efficiency of $100 \%$ [LEY90] and all of the reaction products produced during the bombardment are caught in the foil. In the case of the molybdenum foil, after bombardment the foil was removed from the target chamber and counted directly. The gold foil was chemically processed to remove the gold and other interfering activities before counting.

After the chemical separation, the gold foil was counted for 1030 -min cycles, 12 1-h cycles, 5 10-h cycles and finally $71-\mathrm{d}$ cycles. To determine the yield of the LIM system, ${ }^{248}$ Es and ${ }^{249} \mathrm{Es}$ were identified in the $\alpha$-spectra and their initial activities were 
determined via half-life analyses using the MLDS code. The production cross sections of ${ }^{248}$ Es and ${ }^{249}$ Es were measured to be $1.88 \pm 0.59 \mathrm{mb}$ and $0.54 \pm 0.14 \mathrm{mb}$, respectively. These values agree relatively well with the reported cross sections of approximately 1.8 $\mathrm{mb}$ and $0.8 \mathrm{mb}$ for ${ }^{248} \mathrm{Es}$ and ${ }^{249} \mathrm{Es}$, respectively, at an energy of $18 \mathrm{MeV}$, and $0.68 \mathrm{mb}$ and $0.79 \mathrm{mb}$ for the same nuclides at $23 \mathrm{MeV}$ (see Figure 3.1). To determine the yield of the LIM system, we compared the cross sections measured from the catcher foil to those measured in an excitation function experiment using gas-jet transport to the MG, the results of which will be discussed in section 6.2.3.3. In the excitation function experiment, we measured cross sections for ${ }^{248}$ Es and ${ }^{249}$ Es at a proton energy of $21 \mathrm{MeV}$ assuming a LIM system efficiency of 50\%, exactly as we did during the ECDF experiments. Those cross sections were measured to be $0.19 \pm 0.09 \mathrm{mb}$ and $0.17 \pm 0.08$ $\mathrm{mb}$ for ${ }^{248} \mathrm{Es}$ and ${ }^{249} \mathrm{Es}$, respectively. By comparing results from the excitation function and the catcher foil experiments, the yield of the LIM gas-jet system turned out to be only $5 \pm 2 \%$ in the case of ${ }^{248} \mathrm{Es}$ and $15 \pm 8 \%$ for ${ }^{249} \mathrm{Es}$. These incredibly low yields definitely explain why so few fission events were detected during the ECDF experiments. We had originally assumed the yield was three to 10 times larger than it actually was! Using a similar experimental technique, another researcher determined that the LIM yield during her experiments had only been between $0.5 \%$ and $8 \%$ [LAU98] as well. In both cases, different beam and target combinations were used, which means that no matter what the configuration is the LIM gas-jet transport system appears to have an extremely low yield in these instances.

The difference in yield between ${ }^{248}$ Es and ${ }^{249}$ Es may support the idea presented in section 6.2.3.1.1, that short recoil ranges are preventing reaction products from attaching 
to the $\mathrm{KCl}$ aerosols. ${ }^{248} \mathrm{Es}$ and ${ }^{249} \mathrm{Es}$ have half-lives of $26 \mathrm{~min}$ and $102 \mathrm{~min}$, respectively [FIR96]. If the recoil ranges are smaller than the target thickness, then the reaction products will not make it out of the target. Products with longer half-lives could possibly survive long enough to diffuse out of the target into the stream of the gas-jet. Likewise, shorter-lived products may decay inside the target before they can diffuse out into the gas-jet, resulting in a lower yield for shorter-lived nuclides. Also, the LIM target tests described in section 6.2.3.1.1 indicated that not all $19{ }^{249} \mathrm{Cf}$ targets were contributing to the overall yield of the reaction products. So the unexpectedly small yield of the LIM system may be a combination of things, namely small recoil ranges preventing reaction products from entering the stream of the gas-jet, and the fact that not all of the targets are contributing to the yield of nuclides collected. Whatever the reason for the low LIM yield, it explains the discrepancy between the number of delayed fission events expected during the ECDF experiments and the few that were actually detected.

These results still did not explain why the ${ }^{246}$ Es ECDF experiment seemed to have a larger LIM efficiency than the ${ }^{248}$ Es ECDF experiment. Going back to section 6.1.3, the production cross sections of ${ }^{246} \mathrm{Es},{ }^{247} \mathrm{Es},{ }^{248} \mathrm{Es}$ and ${ }^{249}$ Es were measured at proton energies of $37 \mathrm{MeV}$ and $18 \mathrm{MeV}$ using the newly derived LIM efficiencies of 5\% and $15 \%$ determined from the catcher foil experiment. At $18 \mathrm{MeV}$, a LIM efficiency of 5\% was required for our ${ }^{247}$ Es and ${ }^{248}$ Es experimental cross sections to agree with those reported by Hatsukawa et al. [HAT89] (approximately $30 \mu \mathrm{b}$ and $1.5 \mathrm{mb}$, respectively.) When an efficiency of $15 \%$ was used, our ${ }^{249}$ Es cross section agreed with the Hatsukawa value within error (approximately $0.8 \mathrm{mb}$ [HAT89]). Since $18 \mathrm{MeV}$ is close to $21 \mathrm{MeV}$, it made sense that the LIM efficiencies determined from the catcher foil experiment 
turned out to be the actual efficiency during the ECDF experiment when an $18-\mathrm{MeV}$ proton beam was used. However, the results were quite different at an energy of 37 MeV. Only ${ }^{246}$ Es and ${ }^{247}$ Es had been observed at this energy. For both nuclides, a LIM yield of slightly less than $50 \%$ resulted in cross sections that were the same as those reported in [HAT89] within error (approximately $15 \mu \mathrm{b}$ for ${ }^{246}$ Es and $50 \mu \mathrm{b}$ for ${ }^{247}$ Es.) The reason for this discrepancy lies in the beam energy. As discussed in section 4.1.2 the reaction products recoil out of the target with the same momentum as the incoming projectile. A higher beam energy, therefore, results in a higher momentum imparted to the reaction products. This in turn results in a larger recoil range for the product nuclei, and a larger recoil range means these nuclei travel farther out of the target, making it easier for reaction products to enter the gas-jet, improving the overall yield. So at the highest projectile energies, the LIM system had a maximum yield of 50\%, and at lower beam energies this dropped to $5 \%$ for shorter-lived nuclides. To address the possibility that at lower energies the reaction products were not entering the gas-jet stream, a new capillary system has been designed that brings the stream of the gas-jet closer to the surface of the targets (see Chapter 8).

Unfortunately, the data from the molybdenum catcher foil could not confirm the LIM yields measured from the gold catcher foil. For both ${ }^{248}$ Es and ${ }^{249}$ Es, the cross sections measured from the molybdenum foil were at least an order of magnitude lower than those measured from the gold foil, and did not match previously reported values [HAT89]. The activity level of the foil after irradiation was incredibly high, resulting in a large amount of dead time in the detector. Dead time occurs when the detector is swamped with radiation so that not all of the particles that enter the detector are actually 
counted. This means that too few events are recorded by the data acquisition system, resulting in lower initial activities and smaller cross sections. Therefore, the data from the molybdenum foil were not used in the determination of the yield of the LIM system.

\subsubsection{Effective Target Thickness}

In section 4.1.2 it was noted that compound nuclei produced in reactions with light beams would have very little recoil energy based on conservation of momentum considerations (equation 4.3). A small recoil energy limits the effective thickness of the target to approximately the recoil range of the compound nucleus in the target material. This was the impetus for the design and construction of the LIM target system (see Chapter 4). By simultaneously bombarding several thin targets at once, the overall effective target thickness is increased over that of a single target, increasing the number of nuclei produced in the reaction (see equation 1.3). To determine accurate cross sections from experimental data, it is necessary to know what the overall effective target thickness is for a particular beam energy.

Northcliffe and Schilling [NOR70] have tabulated the ranges of various ions in different materials. For this excitation function experiment, we are interested in the range of ${ }^{250} \mathrm{Es}$ recoils in ${ }^{249} \mathrm{Cf}$ targets. The tables in [NOR70] only go to uranium, but this can be used as a good approximation for californium targets (uranium $\mathrm{Z}=92$, californium $\mathrm{Z}=98$ ) as the ranges of ions through the heavier materials do not vary much with the $\mathrm{Z}$ of the material. To determine the recoil range of a compound nucleus, its recoil energy must be known and can be determined from a variation of equation 4.3: 


$$
E_{\mathrm{CN}}=\frac{m_{\text {proj. }} E_{\text {proj. }}}{m_{\mathrm{CN}}}
$$

where $m$ is the mass number, $E$ is energy, proj. designates projectile and $\mathrm{CN}$ designates compound nucleus. From equation 6.2 , one can see that the einsteinium $\mathrm{CN}$ receives very little recoil energy from a low energy proton beam. For instance, $40-\mathrm{MeV}$ protons result in a ${ }^{250} \mathrm{Es} \mathrm{CN}$ with recoil energy of only $160 \mathrm{keV}$. To determine the range of the $\mathrm{CN}$ at low beam energies, the range data in [NOR70] must be extrapolated to zero recoil energy. Figure 6.19 shows the range data for ${ }^{250} \mathrm{Es}$ in uranium and the resulting best fit to the data calculated with a least squares fitting program. In Figure 6.19 it looks like there may be some deviation between the data from [NOR70] at low energies and the linear fit. Therefore, the extrapolated range values should really be considered as estimates, but can be used for our purposes here. At each proton energy the recoil energy of the $\mathrm{CN}$ changes, and therefore the range of the $\mathrm{CN}$ in the target material changes as well. Once the recoil range of the $\mathrm{CN}$ has been determined for a particular beam energy, the overall effective target thickness for all of the LIM targets together can be determined. The recoil range of the $\mathrm{CN}$ is equal to the effective target thickness of one target. If the actual thickness of a target is less than the recoil range, then the actual target thickness is used instead. The effective target thickness for all of the individual LIM targets are then summed together, resulting in the overall effective target thickness for the whole system. Table 4.1 lists the thickness of the individual ${ }^{249} \mathrm{Cf}$ targets used in the LIM system for the ${ }^{249} \mathrm{Cf}(\mathrm{p}, \mathrm{xn}){ }^{250-\mathrm{x}}$ Es reaction. Hall [HAL89] determined that the average spread in target thickness from one section of a target to another is $7 \%$. This error in target thickness has been included in the overall errors determined for cross sections measured in this dissertation. Table 6.4 summarizes the recoil energies and effective target thickness for 


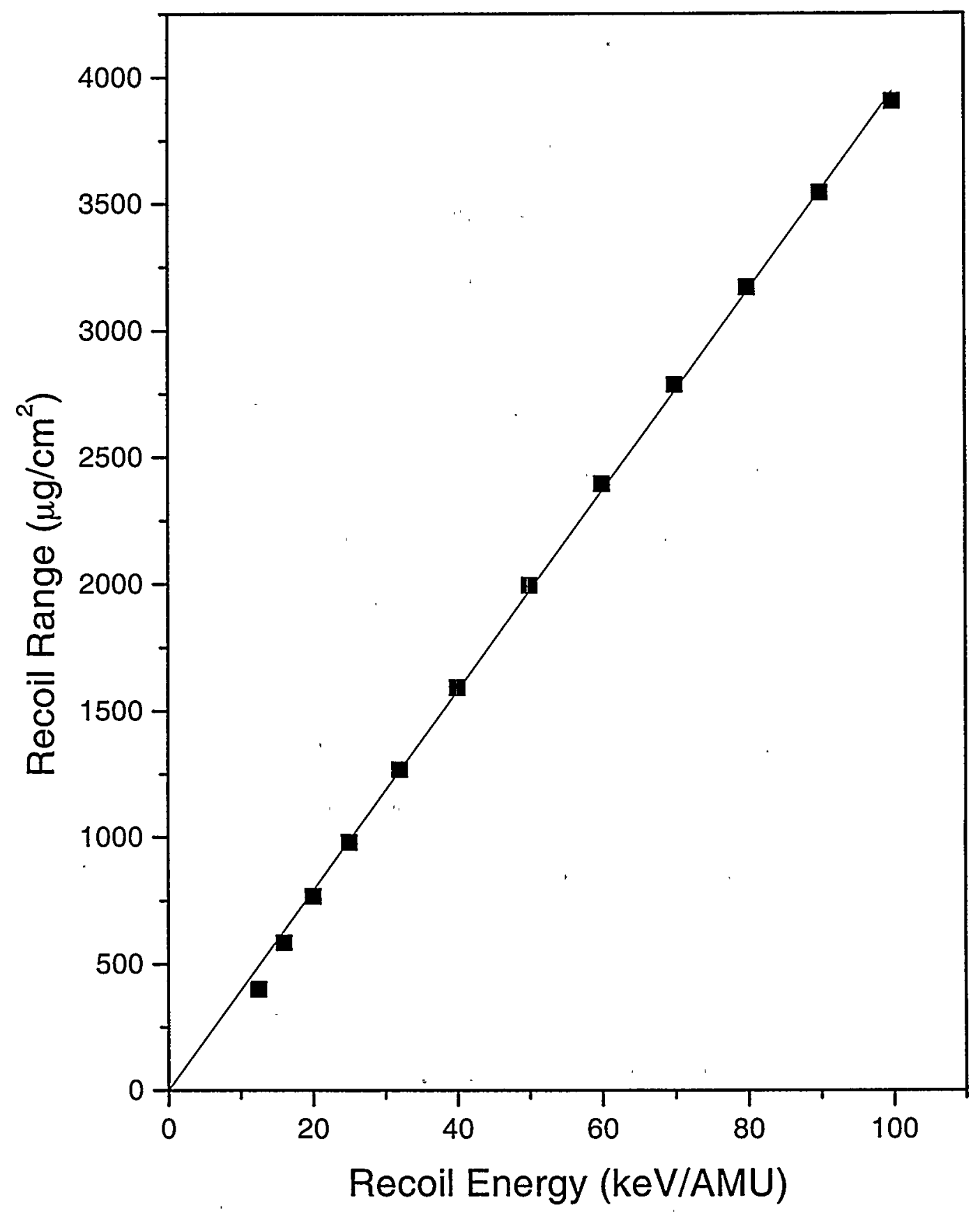

Figure 6.19: Estimation of recoil ranges for the ${ }^{250} \mathrm{Es}$ compound nucleus in uranium by extrapolation of range data in [NOR70] to zero recoil energy. Uranium is used as an approximation of californium, as the ranges of ions in heavier materials do not vary much with the $\mathrm{Z}$ of the material. 
each proton energy in the excitation function experiment and in the ECDF experiments described in section 6.1.3. Also listed in Table 6.4 is the energy loss of proton beams through the LIM system. As described in Chapter 4, the quoted energies of proton beams refer to both the energy of the beam out of the cyclotron and the energy of the beam after passing through the vacuum window and the first Be target backing. The energy loss of the beam listed in Table 6.4 refers to the total amount of energy lost by protons after passing through all $19^{249} \mathrm{Cf}$ targets and target backings and the He gas that flows between the targets.

Table 6.4. Recoil energies of the ${ }^{250}$ Es compound nucleus and overall effective target thickness for each proton energy used during the ${ }^{249} \mathrm{Cf}(\mathrm{p}, \mathrm{xn})^{250-\mathrm{x}} \mathrm{Es}$ excitation function experiment and in ECDF experiments. Recoil energies were determined using equation 6.2 and from Figure 6.19 [NOR70]. The energy losses of the beams through the entire LIM system are also given for reference.

\begin{tabular}{|ccccc|}
\hline $\begin{array}{c}\text { Proton Beam } \\
\text { Energy } \\
(\mathrm{MeV})\end{array}$ & $\begin{array}{c}\text { Energy Loss } \\
\text { of Beam } \\
(\mathrm{MeV})\end{array}$ & $\begin{array}{c}{ }^{250} \text { Es Recoil } \\
\text { Energy } \\
(\mathrm{keV} / \mathrm{A})\end{array}$ & $\begin{array}{c}\text { Single Target } \\
\text { Recoil Range } \\
\left(\mu \mathrm{g} / \mathrm{cm}^{2}\right)\end{array}$ & $\begin{array}{c}\text { Overall Effective } \\
\text { Target Thickness } \\
\left(\mu \mathrm{g} / \mathrm{cm}^{2}\right)\end{array}$ \\
\hline 40 & 0.30 & 0.64 & 26.7 & $265^{*}$ \\
37 & 0.54 & 0.59 & 24.7 & $265^{*}$ \\
35 & 0.66 & 0.56 & 23.4 & $265^{*}$ \\
28 & 1.3 & 0.45 & 18.7 & 264 \\
21 & 2.7 & 0.34 & 14.0 & 242 \\
18 & 3.6 & 0.29 & 12.0 & 219 \\
\hline
\end{tabular}

"Same as the sum of the actual thickness of the individual targets.

\subsubsection{MG Measurements}

As described in section 5.3.2, the ${ }^{249} \mathrm{Cf}(\mathrm{p}, \mathrm{xn}){ }^{250-\mathrm{x}}$ Es excitation function, using 19 ${ }^{249} \mathrm{Cf}$ targets in the LIM system, was studied using a combination of on-line $\alpha$-counting with the MG rotating wheel detection system, and chemical separations of the reaction products, which were then counted for longer time intervals. Both procedures were used for the four proton energies studied during the experiment, $40 \mathrm{MeV}, 35 \mathrm{MeV}, 28 \mathrm{MeV}$ 
and $21 \mathrm{MeV}$ (see section 4.3 for more information.) The energy spread of the projectiles from the vacuum window to the end of the last target through the LIM system ranges from $2.7 \mathrm{MeV}$ at $21 \mathrm{MeV}$ to $0.30 \mathrm{MeV}$ at $40 \mathrm{MeV}$. Table 6.4 gives the energy loss of the proton beam through the LIM system at each energy.

As discussed in section 6.2.3.1, with higher proton energies a gas-jet yield of approximately $50 \%$ for the LIM transport system was required for experimental data to match previously reported cross sections. Based on those results, for proton energies of $40 \mathrm{MeV}, 37 \mathrm{MeV}$ and $35 \mathrm{MeV}$, a $50 \pm 40 \%$ gas-jet efficiency was used during subsequent cross section determinations. This is an approximation at energies higher than $35 \mathrm{MeV}$. The previously reported cross sections in [HAT89] were only measured to $33 \mathrm{MeV}$, and close to this energy the gas-jet of our system had an apparent 50\% yield. The yield at $37 \mathrm{MeV}$ and $40 \mathrm{MeV}$ may be larger, but since we have no data to compare these values to, we are using $50 \%$ as a conservative estimate of the yield. Likewise, the catcher foil experiment described in section 6.2.3.1 used a proton energy of $21 \mathrm{MeV}$ to - determine the yield of the LIM gas-jet transport system. Even though the yield varied slightly with half-life, at this energy the efficiency of the gas-jet was only about $5 \%$. Therefore, at beam energies of $28 \mathrm{MeV}, 21 \mathrm{MeV}$ and $18 \mathrm{MeV}$, a gas-jet efficiency of only $5 \pm 4 \%$ was used to determine cross sections. The yield at $28 \mathrm{MeV}$ may be slightly larger, but again 5\% is used as a conservative estimate of the yield. Because the absolute gas-jet efficiency could not be determined during these experiments, a large error is included on our value for the transfer yield.

Figure 6.20 shows the $\alpha$-spectrum recorded at a proton energy of $40 \mathrm{MeV}$ by the top detector of the second detector pair with a 1-min stepping time in the MG (80 min of 


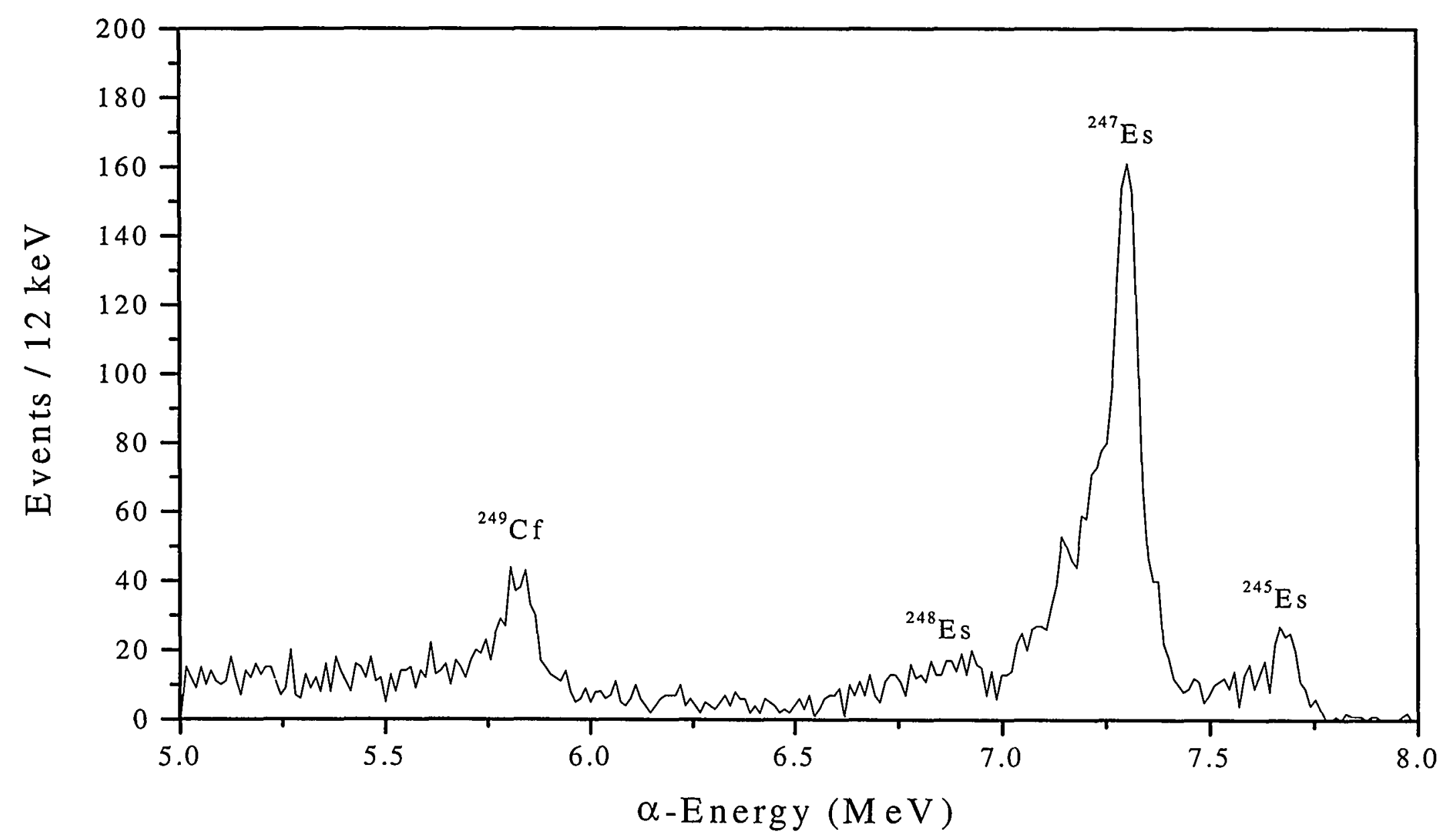

Figure 6.20: $\alpha$-spectrum from the ${ }^{249} \mathrm{Cf}(\mathrm{p}, \mathrm{xn})^{250-\mathrm{x}}$ Es reactions at $40 \mathrm{MeV}$. The spectrum was taken from the top detector of the second detector pair using a 1-min stepping time in the MG (80 min of collection and counting.) The ${ }^{249} \mathrm{Cf}$ is target material that was knocked out by the beam. 
counting.) The $\alpha$-spectra generated at proton energies of $35 \mathrm{MeV}, 28 \mathrm{MeV}$ and $21 \mathrm{MeV}$ looked almost identical to the spectrum seen in Figure $6.20 .{ }^{245} \mathrm{Es},{ }^{247} \mathrm{Es}$ and ${ }^{248} \mathrm{Es}$ were identified in the $40-\mathrm{MeV} \alpha$-spectra recorded with both 1- and 2-min stepping intervals. Even though ${ }^{248}$ Es was observed in these spectra, the chemically processed samples (see section 6.2.3.4) were used to determine its corresponding cross sections because these samples were counted for a longer time interval, covering more of its $26-\mathrm{min}$ half-life. ${ }^{246}$ Es and ${ }^{247}$ Es have similar half-lives and $\alpha$-decay energies, which makes it impossible to resolve the two isotopes over a 6 - or 12-min counting interval (corresponding to 1- or 2-min stepping times.) The large $\alpha$-peak at 7.3 MeV in Figure 6.20 was integrated from 7.0 MeV to 7.4 MeV, resulting in an energy region that included all of the ${ }^{247} \mathrm{Es} \alpha$-groups (see Table 3.2). A half-life analysis of the $\alpha$-peak from both the 1- and 2-min spectra was performed using MLDS. In both cases, the code only identified one component with a half-life of $5.5 \pm 0.3 \mathrm{~min}$, close to $4.6-\mathrm{min}^{247} \mathrm{Es}$. Results from the chemically processed sample at a proton energy of $40 \mathrm{MeV}$ (see section 6.2.3.4) later showed that the amount of ${ }^{246} \mathrm{Es}$ produced at this energy was actually quite small. Therefore, it was assumed that ${ }^{247} \mathrm{Es}$ was the major component of the $7.3-\mathrm{MeV} \alpha$-peak and the decay curve shown in Figure 6.21(a) does indeed appear to have only one component. Based on the decay analysis, the initial activity of ${ }^{247}$ Es after bombardment was $2094 \pm 26$ counts/min. Using a 50\% gas-jet efficiency in equation 6.1 results in a production cross section of 20 $\pm 16 \mu \mathrm{b}$ for ${ }^{247} \mathrm{Es}$ at $40 \mathrm{MeV}$. The SPIT prediction [SIK67] at this energy is approximately $10 \mu \mathrm{b}$, well within the error of our measurement.

A similar analysis was done for the $\alpha$-peak at $7.73 \mathrm{MeV}$ in Figure 6.20. The energy region between $7.6 \mathrm{MeV}$ and $7.8 \mathrm{MeV}$ was integrated to incorporate all of the $\alpha$ - 
groups of ${ }^{245}$ Es. The half-life analysis of the peak using MLDS resulted in one component with a $1.3 \pm 0.1$-min half-life, very close to the reported $1.1 \pm 0.1$-min halflife of ${ }^{245}$ Es. Our half-life determination may be even more accurate than the previously reported value, since the decay of ${ }^{245}$ Es was followed for five half-lives. Figure 6.21(b) shows only one component in the decay of the $\alpha$-peak, which substantiates that the peak is ${ }^{245}$ Es and there are no significant contributions from other components. The initial activity of ${ }^{245}$ Es after bombardment was determined to be $430 \pm 17$ counts/min using both the previously reported half-life and our newly derived value of $1.3 \pm 0.1 \mathrm{~min}$. Using a gas-jet efficiency of $50 \%$ in equation 6.1 results in a production cross section of $0.3 \pm 0.2$ $\mu \mathrm{b}$ for ${ }^{245}$ Es at $40 \mathrm{MeV}$. SPIT predicted a cross section closer to $25 \mu \mathrm{b}$ at this energy, about 80 times larger than what was observed experimentally. SPIT had also predicted a cross section of about $100 \mu \mathrm{b}$ for ${ }^{246}$ Es, 10 times larger than that of ${ }^{247}$ Es. The relative ability of the SPIT code to replicate experimental data will be discussed in section 6.2.4.2, but it is worth mentioning here that in general, SPIT is incapable of predicting cross sections for reactions involving light beams such as protons. The ${ }^{246}$ Es cross section at $40 \mathrm{MeV}$ was measured later to be $3 \pm 2 \mu \mathrm{b}$ from the chemical sample (see section 6.2.3.4), about a factor of three lower than the experimentally determined cross section of $20 \pm 16 \mu \mathrm{b}$ for ${ }^{247}$ Es. This small cross section for ${ }^{246}$ Es supports our initial assumption that the major component of the 7.3-MeV $\alpha$-peak in Figure 6.20 was indeed ${ }^{247}$ Es and that contribution from ${ }^{246}$ Es in comparison was negligible. 

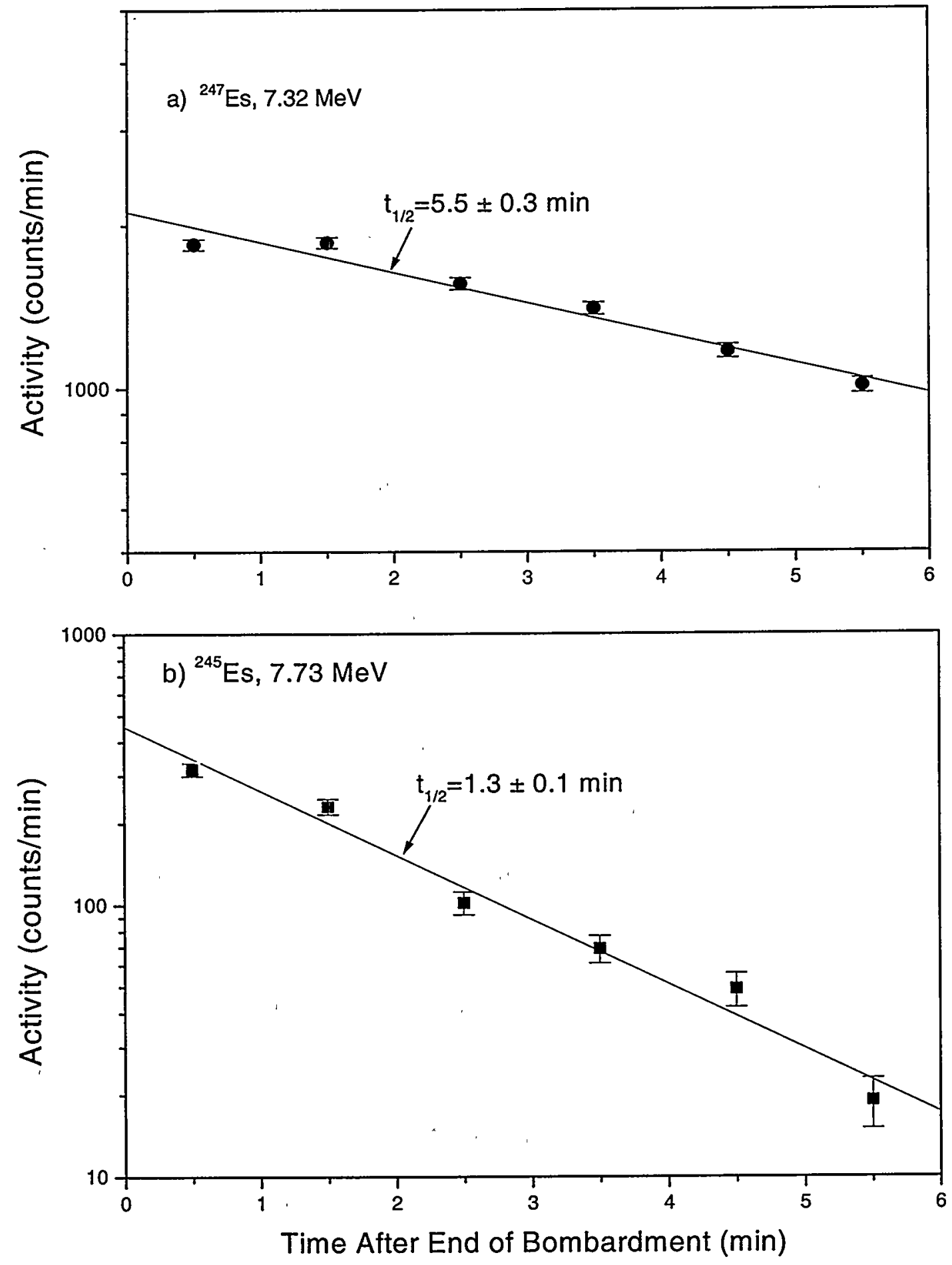

Figure 6.21: Decay of a) 7.32-MeV (7.0 MeV to $7.4 \mathrm{MeV})$ and b) $7.73-\mathrm{MeV}$ (7.6 MeV to $7.8 \mathrm{MeV}$ ) $\alpha$-particles at a projectile energy of $40 \mathrm{MeV}$. The lines represent one-component fits to the data using the MLDS code. The data were collected and counted for $80 \mathrm{~min}$. 
In the $\alpha$-spectrum recorded at a proton energy of $35 \mathrm{MeV}$ there was a small peak at $7.73 \mathrm{MeV}$ indicative of ${ }^{245}$ Es. However, ${ }^{245}$ Es was later observed in the chemically processed samples from this beam energy (see section 6.2.3.4), and a production cross section was calculated from those samples instead because more ${ }^{245}$ Es activity was present. The 7.3-MeV $\alpha$-peak was integrated from $7.0 \mathrm{MeV}$ to $7.4 \mathrm{MeV}$ to incorporate all of the ${ }^{247}$ Es $\alpha$-groups, and a half-life analysis of the peak was performed with MLDS. As before, the program could not resolve the two half-lives of ${ }^{246}$ Es and ${ }^{247}$ Es in the $\alpha$ peak after only 6 or 12 min of counting. The code identified one component with a halflife of $5.2 \pm 0.2 \mathrm{~min}$, close to $4.6-\mathrm{min}{ }^{247} \mathrm{Es}$. Similar to the results at $40 \mathrm{MeV}$, the initial activity of ${ }^{246}$ Es was later determined from the chemical sample (see section 6.2.3.4) to be very small at this energy. Based on the smaller cross section of ${ }^{246}$ Es and the fact that the decay curve of the 7.3-MeV $\alpha$-peak only shows one component (Figure 6.22), it was assumed that ${ }^{247}$ Es was again the major component of the peak. From the decay analysis an initial activity of $1424 \pm 22$ counts/min was determined for ${ }^{247}$ Es after bombardment.

A production cross section of $12 \pm 10 \mu \mathrm{b}$ was measured for ${ }^{247} \mathrm{Es}$ at $35 \mathrm{MeV}$ using a gas-jet efficiency of $50 \pm 40 \%$ in equation 6.1 . The cross section of ${ }^{246} \mathrm{Es}$ was later measured in the chemistry samples and was only $9 \pm 6 \mu \mathrm{b}$ (see section 6.2.3.4.) Even though the cross sections of ${ }^{246}$ Es and ${ }^{247}$ Es are relatively close at this energy, because of its longer half-life, less ${ }^{246}$ Es would be observed in the detectors than ${ }^{247}$ Es. Since there was only one component in the decay curve of the 7.3-MeV $\alpha$-peak, it is safe to assume that ${ }^{247}$ Es is the major component in the peak.

At both $28 \mathrm{MeV}$ and $21 \mathrm{MeV}$, only a large 7.3-MeV $\alpha$-peak is observed in the spectra. At both energies, the $\alpha$-peak was integrated from $7.0 \mathrm{MeV}$ to $7.4 \mathrm{MeV}$, and a 


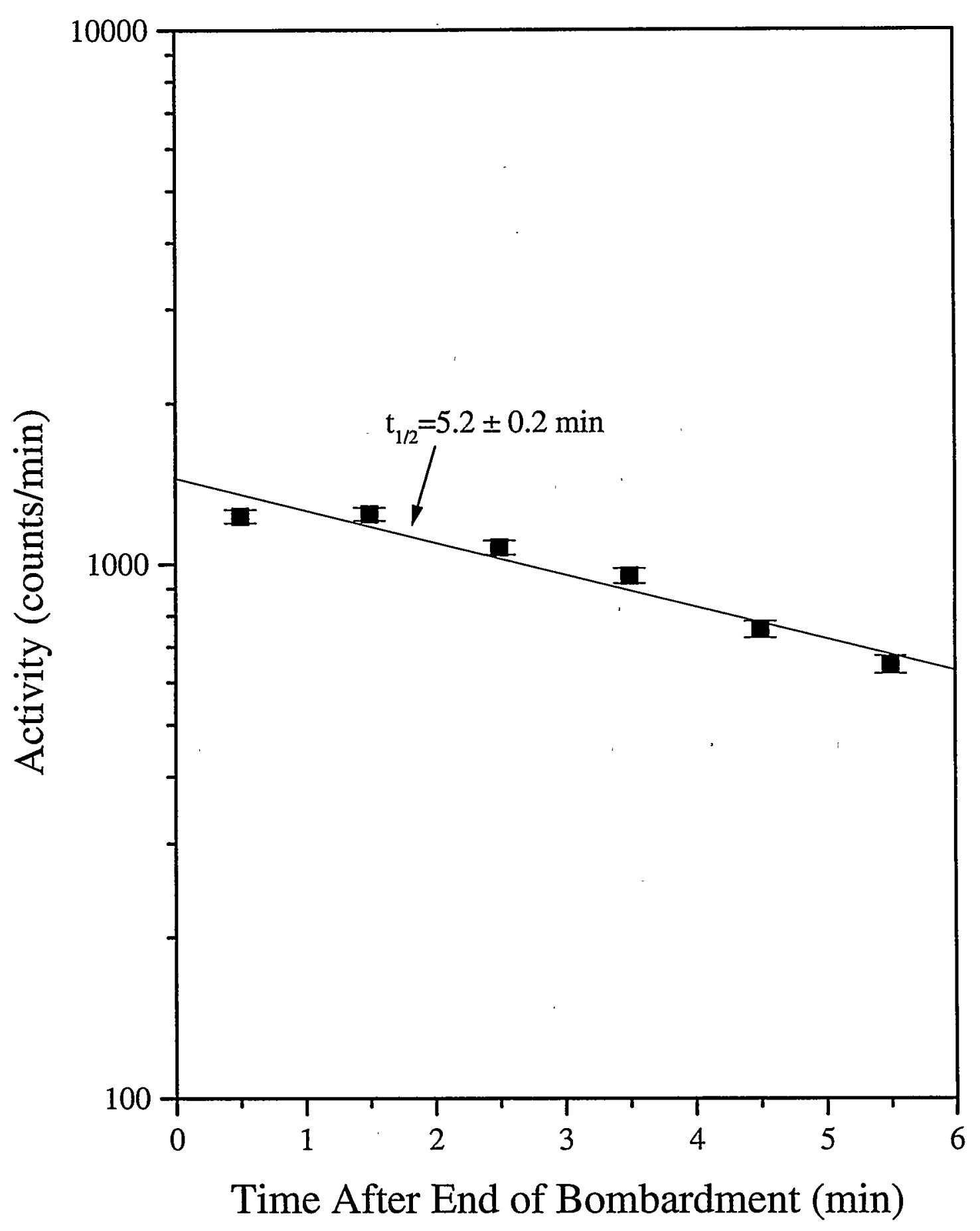

Figure 6.22: Decay of 7.3-MeV $\alpha$-particles integrated from 7.0 MeV to $7.4 \mathrm{MeV}$ at a projectile energy of $35 \mathrm{MeV}$. The line represents a one-component fit to the data using the MLDS code. The data were collected and counted for a total of $80 \mathrm{~min}$. 
half-life analysis was performed using MLDS. Any contribution from ${ }^{246}$ Es was neglected as it was at $40 \mathrm{MeV}$ and $35 \mathrm{MeV}$. As the proton energy decreases, it becomes more difficult for the $4 \mathrm{n}$ exit channel to compete with the $3 \mathrm{n}$ exit channel. Since the initial activities of ${ }^{246} \mathrm{Es}$ had been negligible at $40 \mathrm{MeV}$ and $35 \mathrm{MeV}$, it follows that the initial activities at $28 \mathrm{MeV}$ and $21 \mathrm{MeV}$ would be comparable or even smaller. This was confirmed by the chemical samples, which identified initial activities of ${ }^{246}$ Es that were less than or equal to those seen previously at higher proton energies. The decay curves of the $\alpha$-peak at $28 \mathrm{MeV}$ and $21 \mathrm{MeV}$ only showed evidence of one component, and both looked like the curve seen in Figure 6.22. The half-life analyses identified one component in each case with a half-life of $5.2 \pm 0.2 \mathrm{~min}$. Assuming that ${ }^{247} \mathrm{Es}$ was the major component of the $\alpha$-peak, the initial activity of ${ }^{247}$ Es after bombardment at $28 \mathrm{MeV}$ was $850 \pm 17$ counts/min, and at $21 \mathrm{MeV}$ the initial activity was $454 \pm 12$ counts/min.

Using a $5 \pm 4 \%$ gas-jet yield in equation 6.1 , production cross sections of $0.19 \pm$ $0.15 \mathrm{mb}$ and $0.13 \pm 0.10 \mathrm{mb}$ were calculated for ${ }^{247}$ Es at beam energies of $28 \mathrm{MeV}$ and $21 \mathrm{MeV}$, respectively. In addition, equation 6.1 was used with initial activities of ${ }^{246} \mathrm{Es}$, ${ }^{247} \mathrm{Es},{ }^{248} \mathrm{Es}$ and ${ }^{249} \mathrm{Es}$ determined during the ECDF experiment described in section 6.1.3 to calculate their production cross sections at beam energies of 37 and $18 \mathrm{MeV}$. At 37 $\mathrm{MeV}$ a gas-jet yield of $50 \pm 40 \%$ was used, and a $5 \pm 4 \%$ yield was used for the determinations at $18 \mathrm{MeV}$. At $37 \mathrm{MeV}$ the cross sections of ${ }^{246}$ Es and ${ }^{247}$ Es were measured to be $6 \pm 4 \mu \mathrm{b}$ and $11 \pm 7 \mu \mathrm{b}$, respectively. Likewise, at $18 \mathrm{MeV}$ the cross section of ${ }^{247}$ Es was $11 \pm 9 \mu \mathrm{b},{ }^{248} \mathrm{Es}$ was $1.3 \pm 1.2 \mathrm{mb}$ and the cross section of ${ }^{249} \mathrm{Es}$ was $0.84 \pm 0.67 \mathrm{mb}$ 


\subsubsection{Chemically Separated Samples}

As discussed in section 5.3.2, after one complete revolution of the MG wheel with either a 1- or 2-min stepping interval, a chemical separation was performed on six of the $\mathrm{KCl}$ deposits to remove interfering activities. After the separation, the samples were counted for 1030 -min cycles, 121 -h cycles, 510 -h cycles and finally 71 -d cycles. In each case, the results of the chemical sample collected from 1-min steps were the same as those from the sample derived from 2-min steps. Since more activity was collected with 2-min steps, the results of those separations will be discussed here.

Once the initial activity of a particular nuclide has been determined from a halflife analysis, the corresponding production cross section was determined using a variation of equation 6.1, which takes the chemical efficiency into account:

$$
\sigma=\frac{A_{0}}{\text { chemistry } \cdot \text { yield } \cdot \varepsilon \cdot \mathrm{I} \cdot \mathrm{N} \cdot \mathrm{e}^{-\lambda \cdot \mathrm{t}_{\text {trans }}} \cdot\left(1-\mathrm{e}^{-\lambda \cdot \mathrm{t}_{\mathrm{ir}}}\right)}
$$

where $A_{o}$ is the initial activity of the nuclide of interest at the end of bombardment (counts/time), and chemistry is the efficiency of the chemical separation. When $\mathrm{A}_{\mathrm{o}}$ is determined from the half-life analysis, the elapsed time between the end of bombardment and the start of the counting interval (the time of the chemical procedure) is taken into account. The other symbols were explained previously. The chemical efficiency was determined by comparing the amount of ${ }^{249} \mathrm{Cf}$ in one $\mathrm{KCl}$ deposit to the total amount seen in the chemical sample correcting for the number of foils.

As discussed in the previous section, ${ }^{246}$ Es could not be identified in the MG data because the MLDS code could not resolve the similar half-lives of ${ }^{246}$ Es and ${ }^{247}$ Es over the short counting times used in the MG. Fortunately, we were able to measure cross sections for ${ }^{246} \mathrm{Es}$ at each energy using the chemistry samples. ${ }^{246} \mathrm{Cf}$, the EC-daughter of 
${ }^{246} \mathrm{Es}$, was observed in all of the $\alpha$-spectra from the chemistry samples. ${ }^{246} \mathrm{Es}$ has a known EC-branch of about $90 \%$ [FIR96], which means that by calculating the initial activity of ${ }^{246} \mathrm{Cf}$ we can subsequently determine the initial activity of ${ }^{246}$ Es and its production cross section.

The ${ }^{246} \mathrm{Cf} 6.75-\mathrm{MeV} \alpha$-peak was integrated from $6.65 \mathrm{MeV}$ to $6.80 \mathrm{MeV}$ in the $\alpha$-spectra from samples produced at proton energies of $40 \mathrm{MeV}$ and $35 \mathrm{MeV}$. The decay curves at both projectile energies showed only one component (Figure 6.23). A onecomponent half-life analysis of the peaks was performed with the MLDS code. At both $40 \mathrm{MeV}$ and $35 \mathrm{MeV}$, the analysis identified only one component in the peak with halflives of $36.3 \pm 0.5 \mathrm{~h}$ and $35.1 \pm 0.6 \mathrm{~h}$, respectively. These values are both very close to the reported $35.7 \pm 0.5$-h half-life of ${ }^{246} \mathrm{Cf}$. The initial activities of ${ }^{246} \mathrm{Cf}$ at $40 \mathrm{MeV}$ and $35 \mathrm{MeV}$ were $526 \pm 5$ counts $/ \mathrm{h}$ and $158 \pm 2$ counts/h, respectively. These values subsequently translated into initial activities of ${ }^{246}$ Es of $584 \pm 6$ counts/h and $176 \pm 3$ counts/h. Direct production of ${ }^{246} \mathrm{Cf}$ via the ${ }^{249} \mathrm{Cf}(\mathrm{p}, \mathrm{p} 3 \mathrm{n})$ reaction was neglected. As discussed in Chapter 1, deexcitation of the compound nucleus via neutron emission is more probable than charged particle emission because of the Coulomb barrier of the nucleus. The p3n exit channel was estimated to be less than $10 \%$ of the $4 n$ exit channel [GAN80, HEN90], which is well within the standard deviation of our measurements. In fact, the large errors associated with the uncertainty of the yield of the gas-jet transport system more than outweigh any contribution from proton out exit channels. For both energies, a gas-jet yield of $50 \pm 40 \%$ was used in subsequent cross section determinations. The efficiencies of the chemical separations were approximately $57 \%$ for the $40-\mathrm{MeV}$ samples and $47 \%$ for the $35-\mathrm{MeV}$ samples. Using these chemical and gas- 


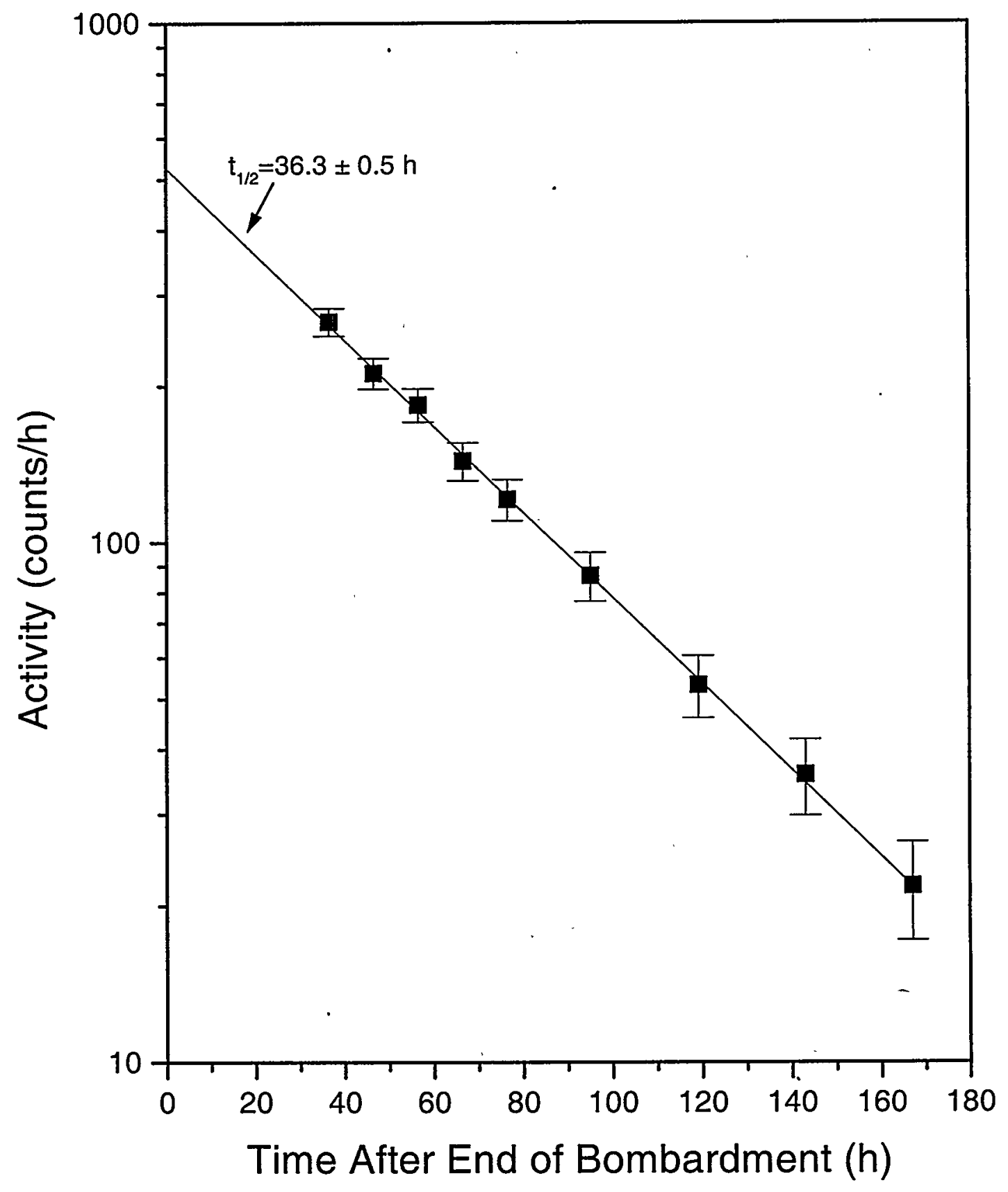

Figure 6.23: Decay of 6.75-MeV $\alpha$-particles integrated from $6.65 \mathrm{MeV}$ to $6.80 \mathrm{MeV}$ at a projectile energy of $40 \mathrm{MeV}$. The line represents a onecomponent fit to the data using the MLDS code. The decay of the same $\alpha$-peak from the $35-\mathrm{MeV}$ proton bombardment has a similar shape. The data were collected for $12 \mathrm{~min}$ and counted over several days. 
jet efficiencies in equation 6.3 , production cross sections of $3 \pm 2 \mu \mathrm{b}$ and $9 \pm 6 \mu \mathrm{b}$ were measured for ${ }^{246} \mathrm{Es}$ at proton energies of $40 \mathrm{MeV}$ and $35 \mathrm{MeV}$, respectively.

The decay curve of the ${ }^{246} \mathrm{Cf} 6.7-\mathrm{MeV} \alpha$-peak at projectile energies of $28 \mathrm{MeV}$ and $21 \mathrm{MeV}$ was integrated from $6.65 \mathrm{MeV}$ to $6.80 \mathrm{MeV}$ and showed two components, a very short component and a much longer-lived isotope (Figure 6.24). A two-component half-life analysis of the data using MLDS identified the two isotopes in the $\alpha$-peaks as $1.7-\mathrm{h}{ }^{249} \mathrm{Es}$ and $35.7-\mathrm{h}{ }^{246} \mathrm{Cf}$. The half-life analyses resulted in initial activities for both ${ }^{249} \mathrm{Es}$ and ${ }^{246} \mathrm{Cf}$, and therefore ${ }^{246} \mathrm{Es}$. At $28 \mathrm{MeV}$ the initial activity of ${ }^{249} \mathrm{Es}$ was $157 \pm 21$ counts/h and the initial activity of ${ }^{246} \mathrm{Cf}$ was $100 \pm 2$ counts/h (111 \pm 3 counts/h for ${ }^{246} \mathrm{Es}$. $)$ At $21 \mathrm{MeV}$ the initial activities were $119 \pm 12$ counts/h and $10.9 \pm 0.6$ counts $/ \mathrm{h}$ for ${ }^{249} \mathrm{Es}$ and ${ }^{246} \mathrm{Cf}$, respectively, resulting in an initial ${ }^{246} \mathrm{Es}$ activity of $12.1 \pm 0.8$ counts/h. The efficiencies of the chemical separations were approximately $66 \%$ at $28 \mathrm{MeV}$ and $50 \%$ for the $21 \mathrm{MeV}$ sample. Using these chemical yields and a gas-jet efficiency of $5 \pm 4 \%$ in equation 6.3 resulted in production cross sections for both ${ }^{249} \mathrm{Es}$ and ${ }^{246} \mathrm{Es}$. At $28 \mathrm{MeV}$, the cross section of ${ }^{249}$ Es was $1.5 \pm 1.2 \mathrm{mb}$ and ${ }^{246}$ Es was $10 \pm 8 \mu \mathrm{b}$. Likewise, at 21 $\mathrm{MeV}$ the cross sections for ${ }^{249} \mathrm{Es}$ and ${ }^{246} \mathrm{Es}$ were measured to be $5.4 \pm 1.4 \mathrm{mb}$ and $2.0 \pm 1.0 \mu \mathrm{b}$, respectively. The relatively small cross sections measured for ${ }^{246} \mathrm{Es}$ at each proton energy support the assumptions made in section 6.2 .3 .3 that ${ }^{247}$ Es was the major component of the 7.3-MeV $\alpha$-peak, and the contribution from ${ }^{246} \mathrm{Es}$ was negligible in comparison.

In the chemically separated samples from $35-\mathrm{MeV}$ protons, ${ }^{245} \mathrm{Cf}$ (the ECdaughter of ${ }^{245} \mathrm{Es}$ ) was clearly observed. The direct production of ${ }^{245} \mathrm{Cf}$ via the ${ }^{249} \mathrm{Cf}(\mathrm{p}, \mathrm{p} 4 \mathrm{n}){ }^{245} \mathrm{Cf}$ reaction was neglected. Unfortunately, the computer that was 

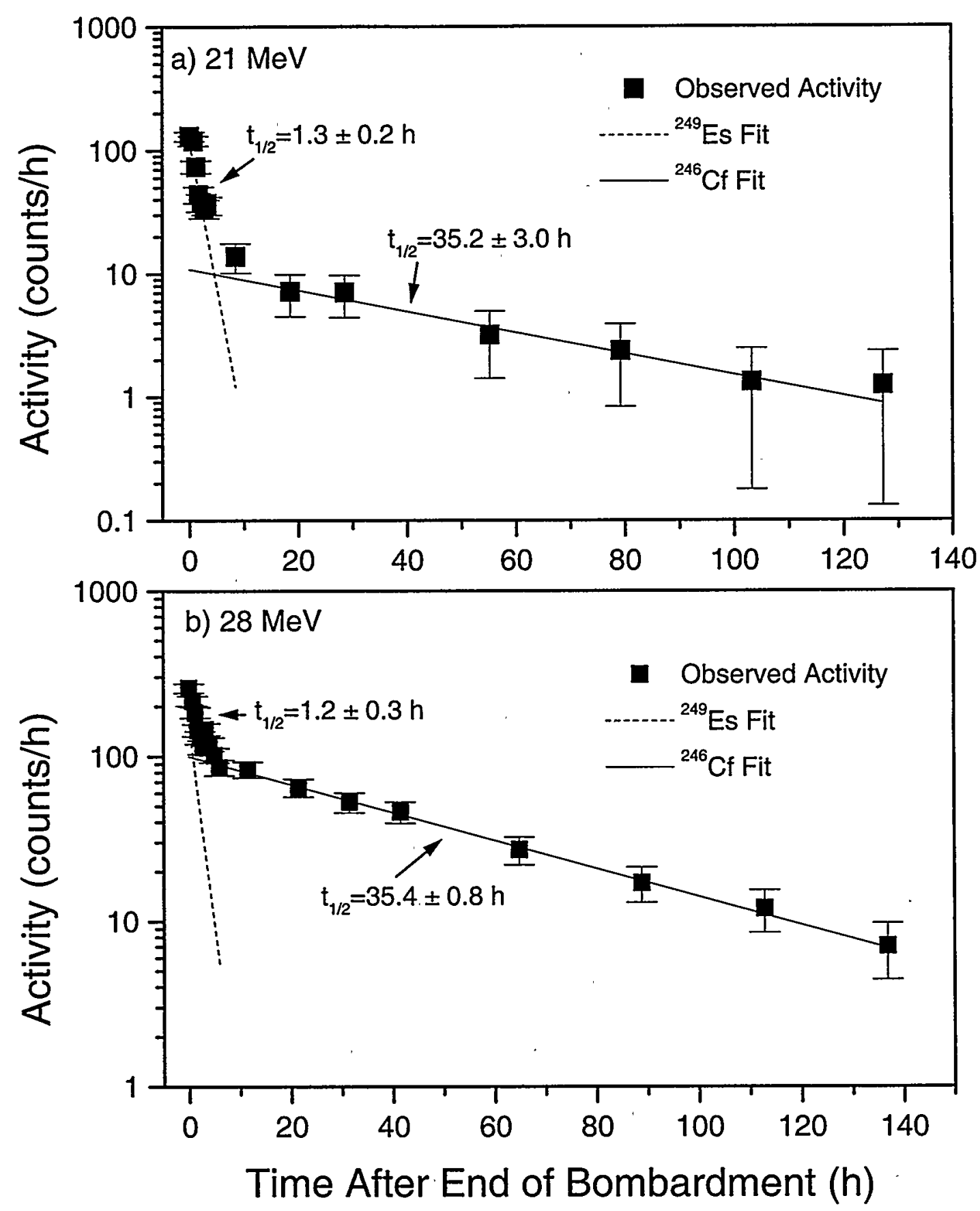

Figure 6.24: Decay of 6.7-MeV $\alpha$-particles integrated from $6.65 \mathrm{MeV}$ to 6.80 $\mathrm{MeV}$ at a projectile energy of a) $21 \mathrm{MeV}$ and b) $28 \mathrm{MeV}$. The lines represent two-component fits to the data using the MLDS code. The data were collected for $12 \mathrm{~min}$ and counted over several days. 
collecting data during the counting of the $35 \mathrm{MeV}$ chemical sample unexpectedly stopped working after taking only a few data points. When a half-life analysis was done on these points with MLDS, the half-life did match the reported 45 -min half-life of ${ }^{245} \mathrm{Cf}$ reasonably well (approximately $50 \mathrm{~min}$.) An energy region from $7.05 \mathrm{MeV}$ to $7.20 \mathrm{MeV}$ was integrated for the half-life analysis. An initial activity of ${ }^{245} \mathrm{Cf}$ was calculated from the half-life analysis by extrapolating the data back to time zero, taking the time between the end of collection and the beginning of counting into account. From this activity and the known $60 \%$ EC-branch of ${ }^{245} \mathrm{Es}$, an initial activity of ${ }^{245} \mathrm{Es}$ of $3432 \pm 50$ counts/h was determined. At $35 \mathrm{MeV}$, the chemical separation had a yield of approximately $47 \%$. Using this yield and a gas-jet efficiency of $50 \%$ in equation 6.3 resulted in a cross section of $0.05 \pm 0.04 \mu \mathrm{b}$ for ${ }^{245} \mathrm{Es}$ at $35 \mathrm{MeV}$.

The only other nuclide observed in the chemistry samples was ${ }^{248}$ Es. Unfortunately, the cross section at $35 \mathrm{MeV}$ could not be determined because of the computer failure mentioned previously. However, ${ }^{248}$ Es was detected in the other three samples at an $\alpha$-energy of $6.87 \mathrm{MeV}$. At $40 \mathrm{MeV}, 28 \mathrm{MeV}$ and $21 \mathrm{MeV}$, the $\alpha$-peak at 6.87 MeV was integrated from $6.80 \mathrm{MeV}$ to $7.20 \mathrm{MeV}$ and then analyzed with the MLDS code. Only one component was identified. Figure 6.25 shows a representative decay curve generated from the $40-\mathrm{MeV}$ sample. The chemical efficiency and gas-jet efficiency at each proton beam energy have been mentioned previously.

For each sample, the decay curve was extrapolated back to time zero to determine the initial activity of ${ }^{248} \mathrm{Es}$. At $40 \mathrm{MeV}$ it was $329 \pm 32$ counts/h, for $28 \mathrm{MeV}$ it was $178 \pm 21$ counts/h and at a projectile energy of $21 \mathrm{MeV}$ is was $358 \pm 35$ counts/h. Once again, a $50 \pm 40 \%$ gas-jet yield was used at $40 \mathrm{MeV}$ and a $5 \pm 4 \%$ efficiency was used for 


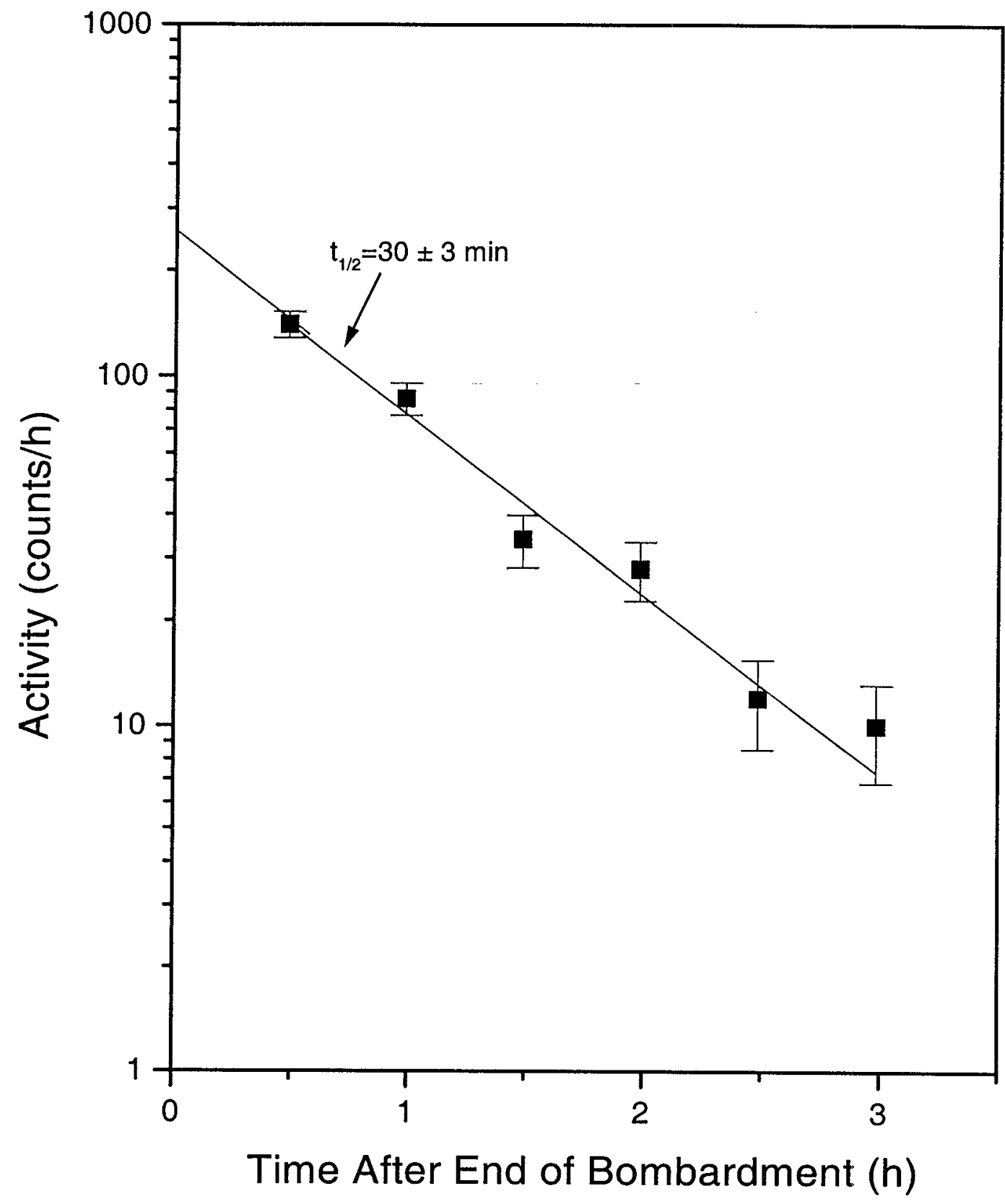

Figure 6.25: Decay of 6.87-MeV $\alpha$-particles integrated from $6.80 \mathrm{MeV}$ to $7.0 \mathrm{MeV}$ at a projectile energy of $40 \mathrm{MeV}$. The line represents a one-component fit to the data using the MLDS code. The decay curves taken from the $\alpha$-data at proton energies of 28 $\mathrm{MeV}$ and $21 \mathrm{MeV}$ have a similar shape. The data were collected for $12 \mathrm{~min}$ and counted for several days. 
both 28 and $21 \mathrm{MeV}$. Putting all of these factors into equation 6.3 resulted in the production cross section for ${ }^{248} \mathrm{Es}$ at each energy. At $40 \mathrm{MeV}, 28 \mathrm{MeV}$ and $21 \mathrm{MeV}$ the cross section was $0.10 \pm 0.09 \mathrm{mb}, 0.94 \pm 0.75 \mathrm{mb}$ and $1.9 \pm 0.59 \mathrm{mb}$, respectively.

\subsubsection{Summary of Cross Sections}

All of the production cross sections determined from the ${ }^{249} \mathrm{Cf}(\mathrm{p}, \mathrm{xn}){ }^{250-\mathrm{x}} \mathrm{Es}$ reactions both through on-line measurements and the counting of chemically separated samples are presented in Figures $6.26\left({ }^{245} \mathrm{Es}\right), 6.27\left({ }^{246} \mathrm{Es}\right), 6.28\left({ }^{247} \mathrm{Es}\right), 6.29\left({ }^{248} \mathrm{Es}\right)$ and $6.30\left({ }^{249} \mathrm{Es}\right)$. In addition, the SPIT prediction [SIK67] for each reaction is included on the respective figures for comparative purposes. Even though it appears in Figure 6.28 that the excitation function for the $3 \mathrm{n}$ exit channel is increasing at higher proton energies, it is probably due to the large uncertainty in the gas-jet yield, and the error bars on the high energy points should be taken into account.

Figure 6.31 shows all of the cross sections determined in this excitation function experiment, along with the values previously reported by Hatsukawa et al. [HAT89]. ${ }^{246}$ Es and ${ }^{247}$ Es show excellent agreement with their published cross sections, but there is more deviation between our results and those of Hatsukawa for ${ }^{248}$ Es and ${ }^{249}$ Es, especially at higher proton energies. Overall, the agreement has to be considered relatively good, especially when the errors are taken into account. 


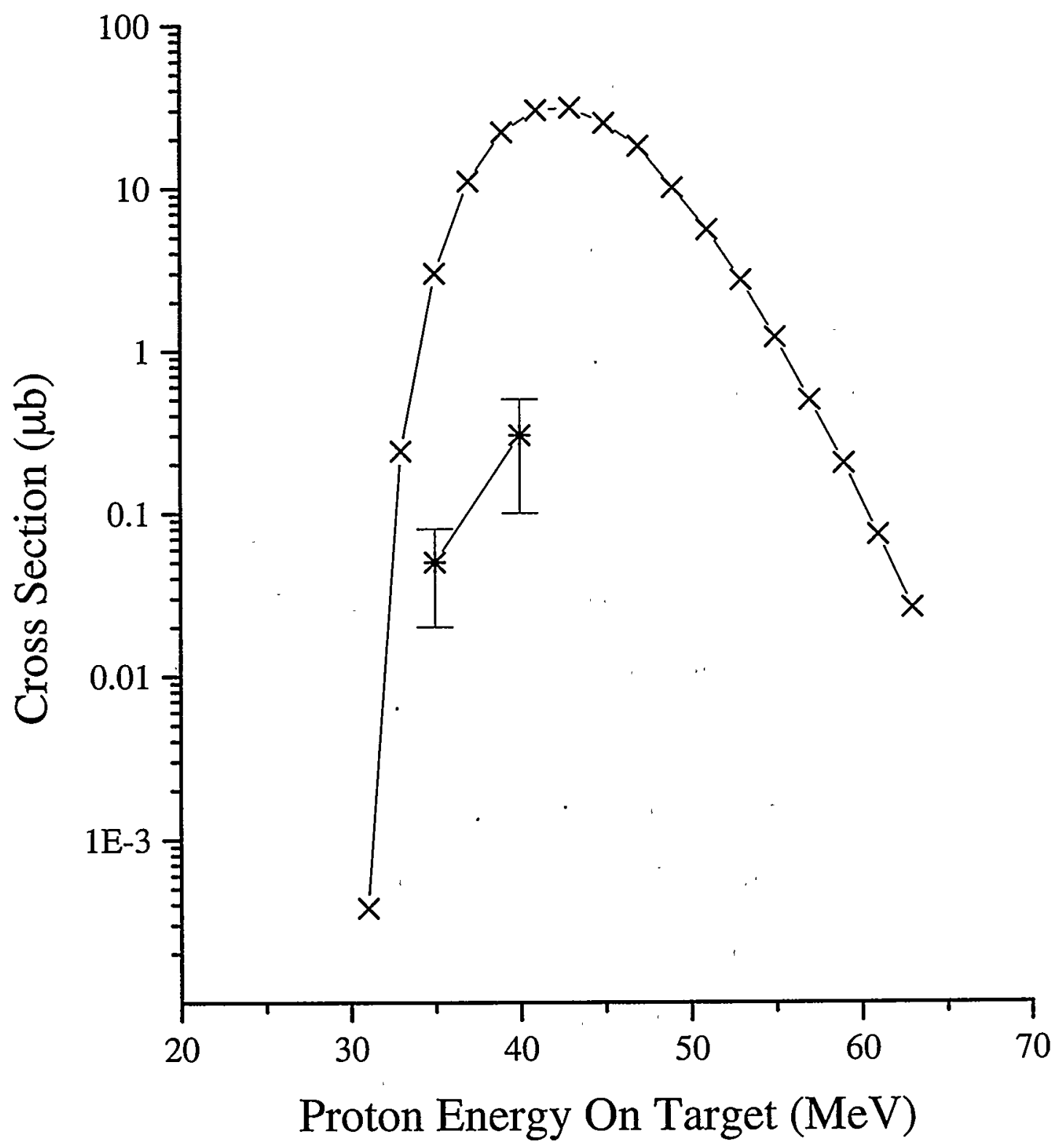

Figure 6.26: Experimentally determined cross sections for the ${ }^{249} \mathrm{Cf}(\mathrm{p}, 5 \mathrm{n}){ }^{245}$ Es reaction (star symbols) and the SPIT prediction ( $\mathrm{x}$ symbols [SIK67]) for the same reaction. 


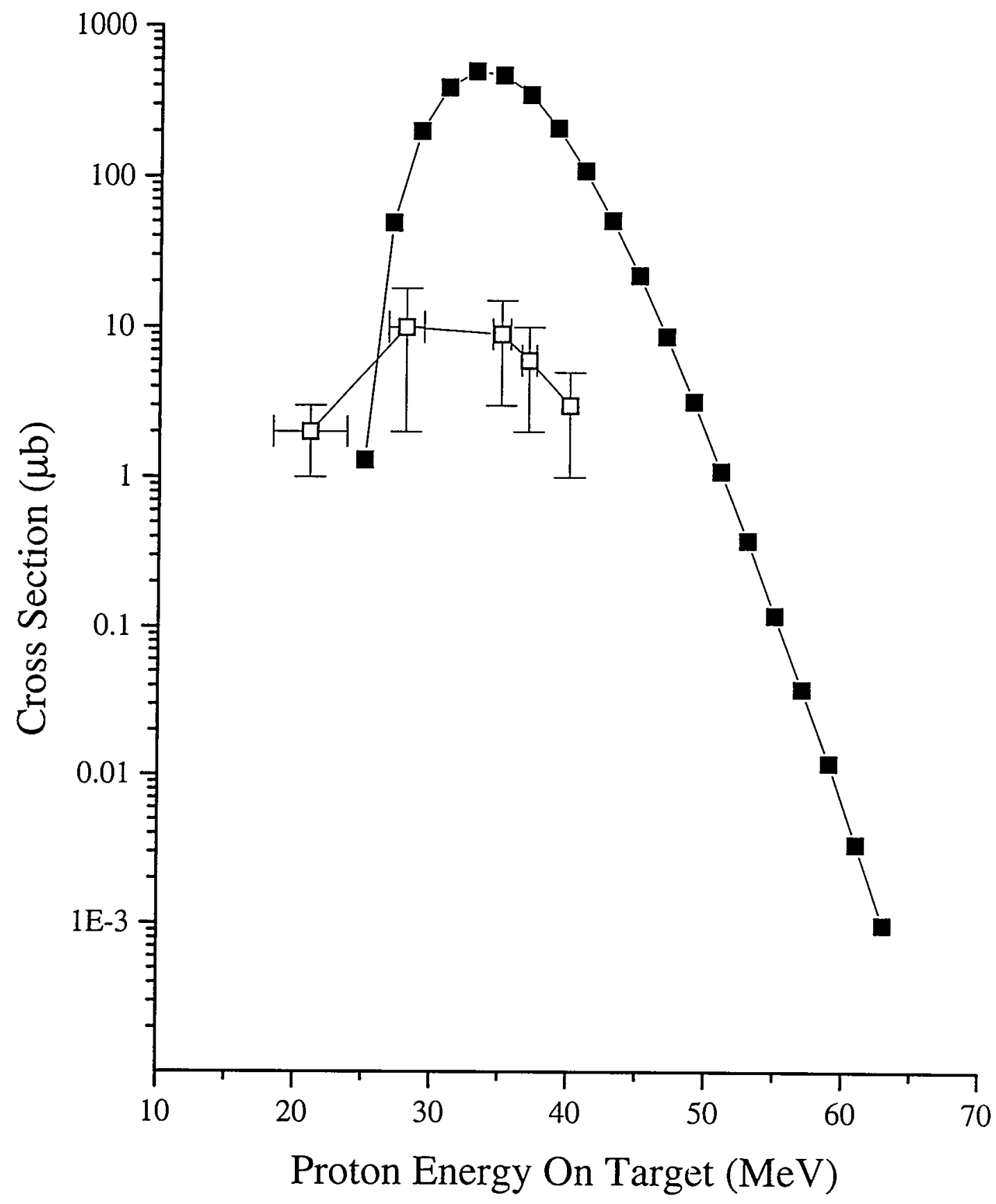

Figure 6.27: Experimentally determined cross sections for the ${ }^{249} \mathrm{Cf}(\mathrm{p}, 4 \mathrm{n}){ }^{246}$ Es reaction (open squares) and the SPIT prediction (closed squares [SIK67]) for the same reaction. 


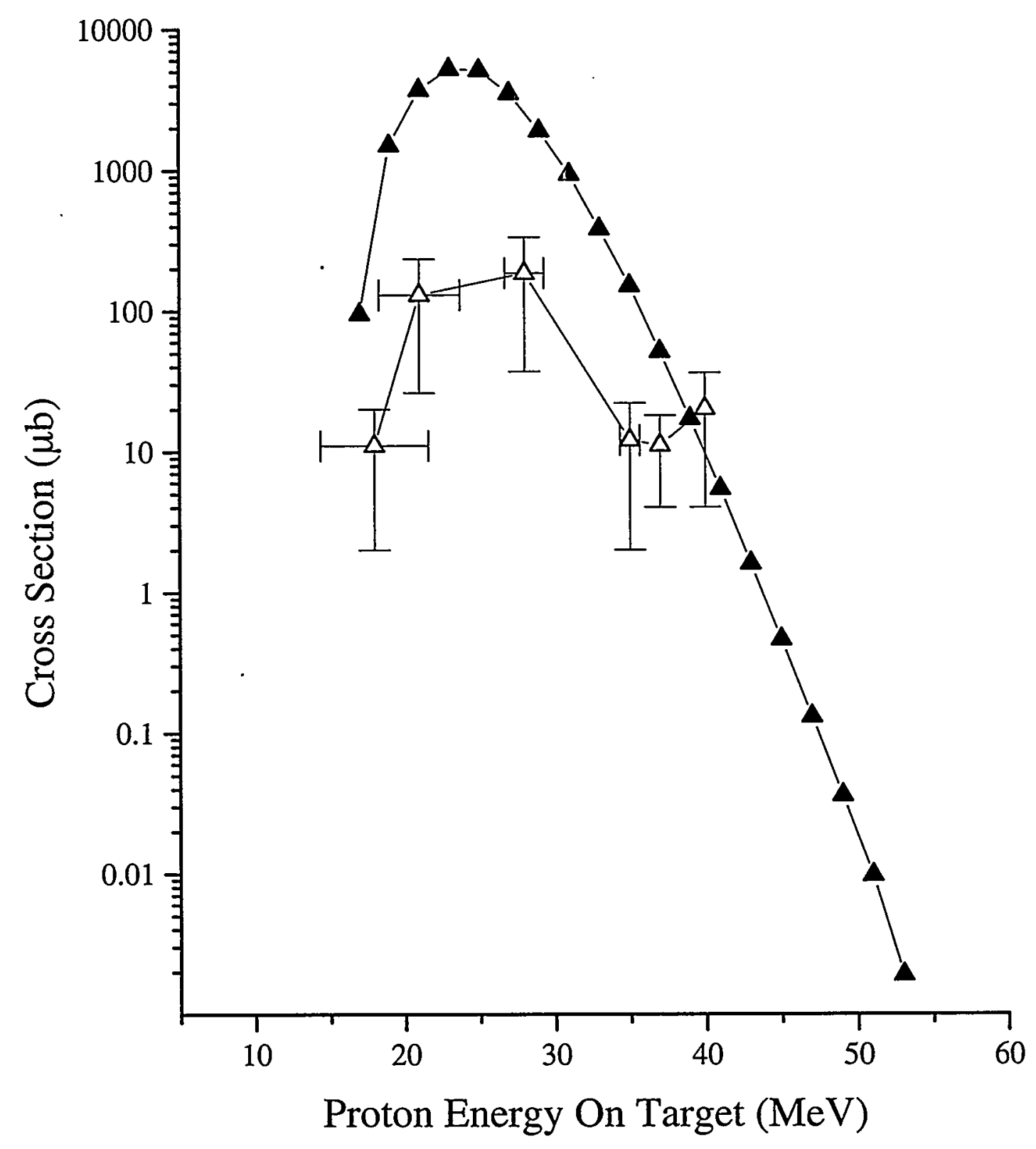

Figure 6.28: Experimentally determined cross sections for the ${ }^{249} \mathrm{Cf}(\mathrm{p}, 3 \mathrm{n}){ }^{247}$ Es reaction (open triangles) and the SPIT predictions (closed triangles [SIK67]) for the same reaction. 


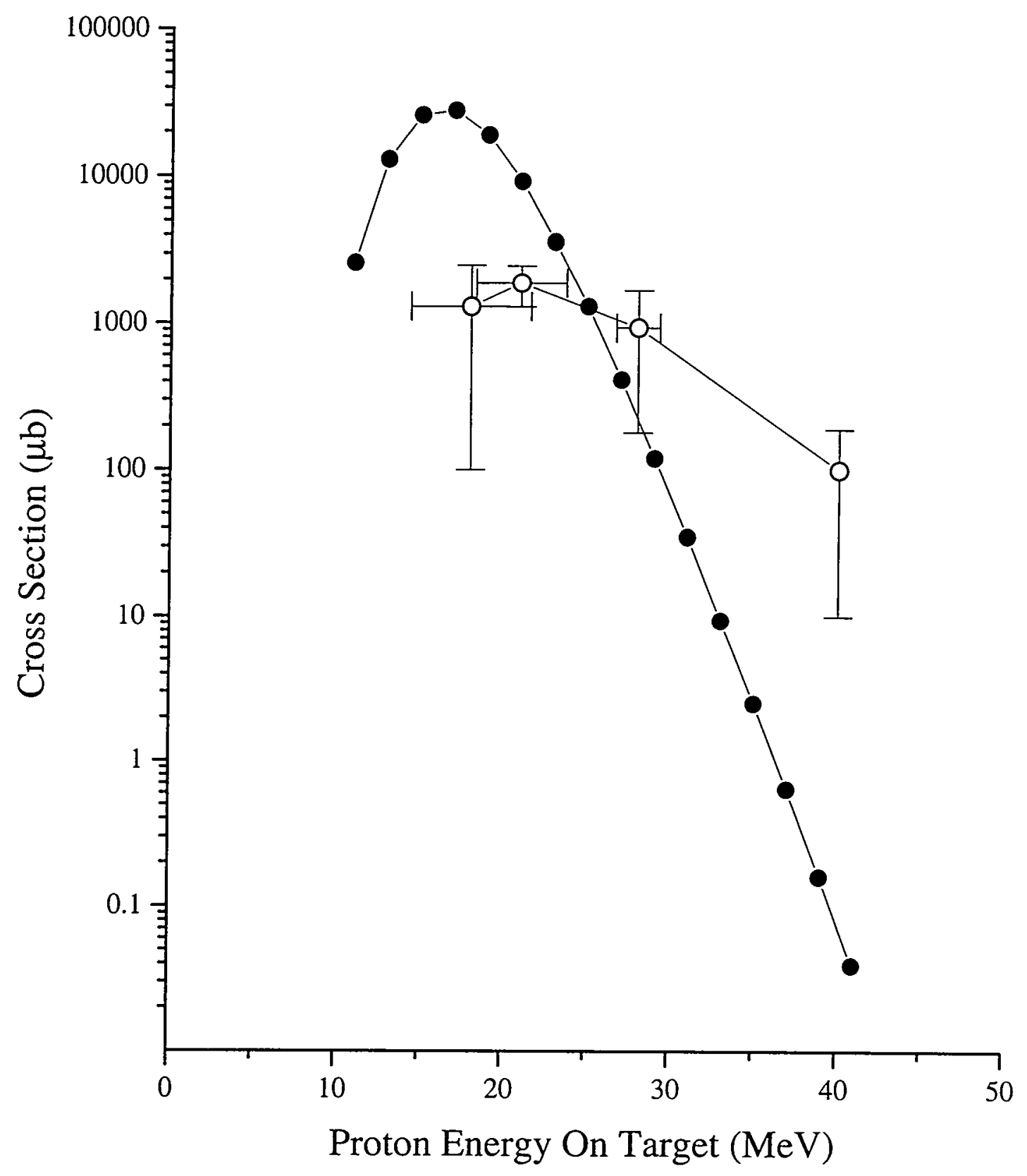

Figure 6.29: Experimentally determined cross sections for the ${ }^{249} \mathrm{Cf}(\mathrm{p}, 2 \mathrm{n}){ }^{248} \mathrm{Es}$ reaction (open circles) and the SPIT predictions (closed circles [SIK67]) for the same reaction. 


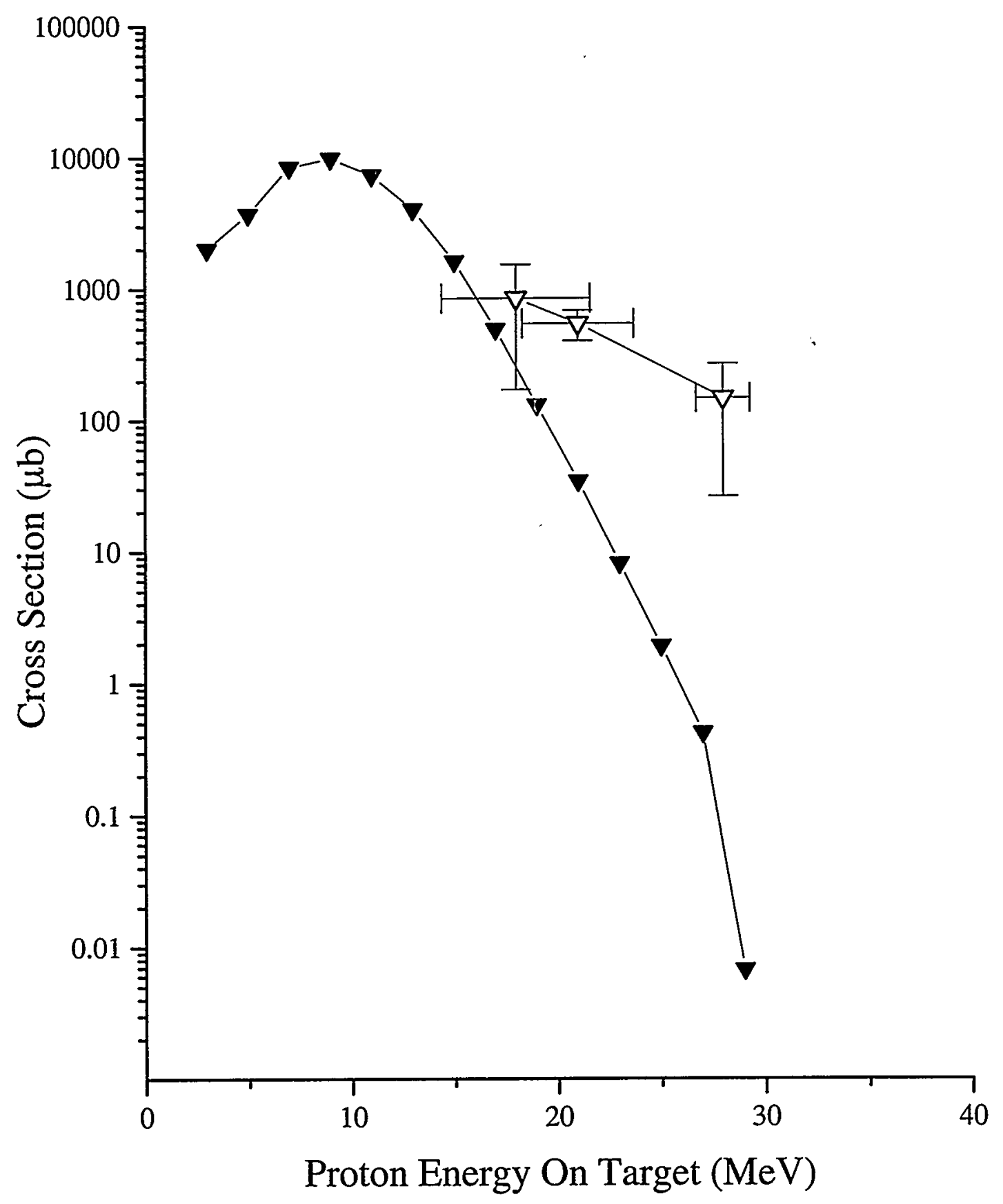

Figure 6.30: Experimentally determined cross sections for the ${ }^{249} \mathrm{Cf}(\mathrm{p}, 1 \mathrm{n}){ }^{249} \mathrm{Es}$ reaction (open triangles) and the SPIT predictions (closed triangles [SIK67]) for the same reaction. 


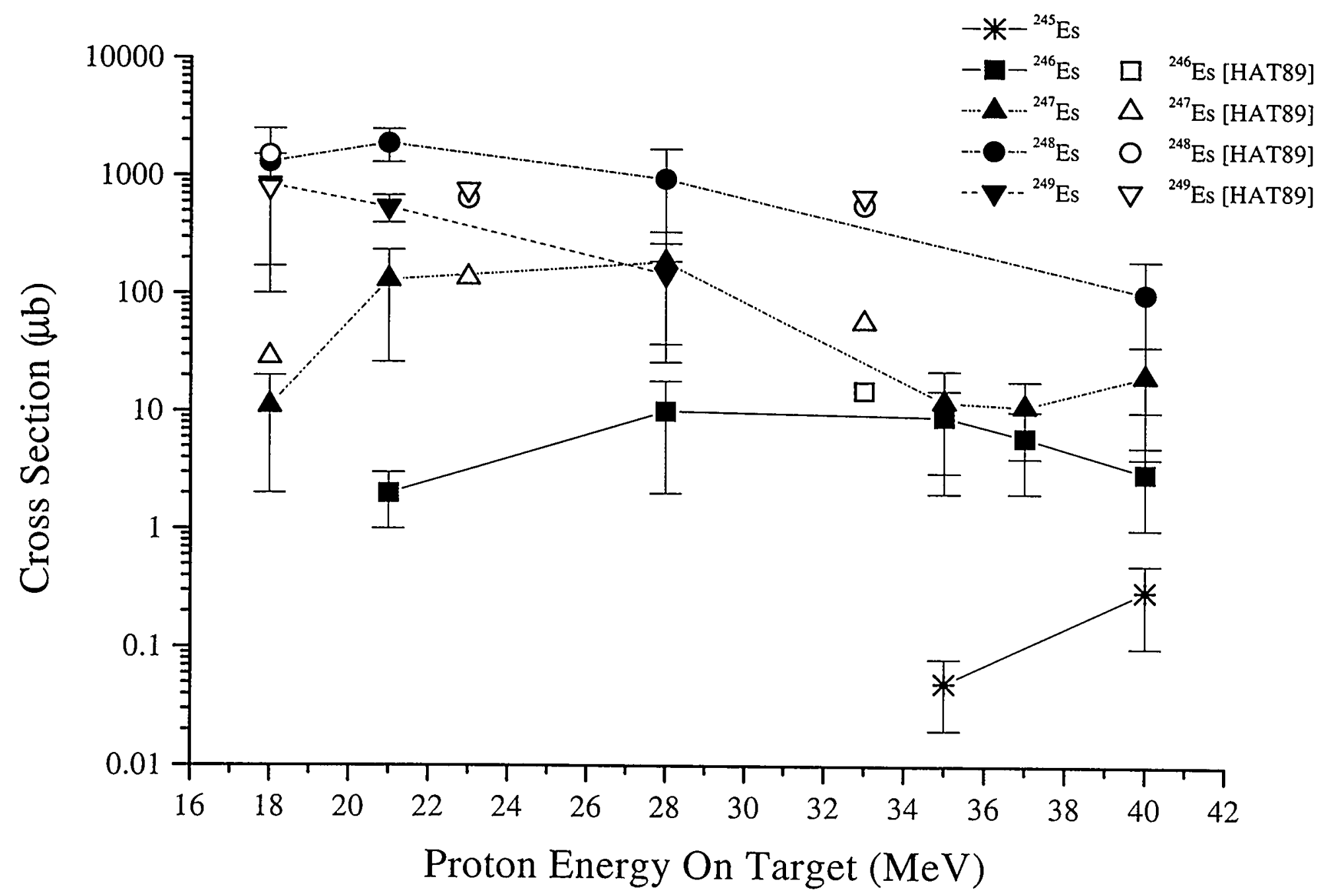

Figure 6.31: Experimentally determined cross sections for the ${ }^{249} \mathrm{Cf}(\mathrm{p}, \mathrm{xn}){ }^{250-\mathrm{x}}$ Es reactions. For comparison, the cross sections reported by Hatsukawa et al. [HAT89] are also shown as open symbols. The uncertainties in beam energy have been omitted for clarity. Refer to Table 6.4 for the energy losses of the proton beams. 


\subsubsection{The SPIT Code for Calculation of Cross Sections}

\subsubsection{The Model}

The compound nucleus model, briefly mentioned in Chapter 1 , is the basis of the SPIT cross section prediction code [SIK67]. In the model, it is assumed that the incident particle enters the target, fusing completely with it to form the compound nucleus. The kinetic energy of the incident particle is then distributed randomly among a sufficiently large number of nucleons, forming an excited, quasi-stationary state [FRI81]. The compound nucleus will deexcite either via fission or by the evaporation of nucleons. As discussed in Chapter 1, once the compound-nucleus is formed,-it is assumed to completely forget its method of production, and the probability of decay into a specific set of final products depends only on the total energy and angular momentum of the system. Detailed reviews of the compound nucleus model have been published [HOD67, THO68], and the reader is referred to them for more detailed information concerning the theory behind the model.

The total reaction cross section $(\sigma)$ calculated by SPIT using the compound nucleus model can be expressed by equation 6.4 :

$$
\sigma=\sigma_{\mathrm{CN}} \mathrm{W}_{\text {prod }}
$$

where $\sigma_{\mathrm{CN}}$ is the cross section for the formation of the compound nucleus $(\mathrm{CN})$ and $\mathrm{W}_{\text {prod }}$ is the probability that the $\mathrm{CN}$ will decay into the desired products. Fission or neutron emission is the most probable mode of decay of the $\mathrm{CN}$ so that all other decay modes can be neglected. The probability that the $\mathrm{CN}$ will decay via emission of a particular number of neutrons can be expressed in terms of the partial widths for decay by fission and neutron emission, 


$$
\mathrm{W}_{\text {prod }}=\prod_{\mathrm{i}=1}^{\mathrm{x}} \frac{\Gamma_{\mathrm{n}}}{\left(\Gamma_{\mathrm{n}}+\Gamma_{\mathrm{f}}\right)_{\mathrm{i}}}
$$

where $\Gamma_{\mathrm{n}}$ is the partial width for decay via neutron emission, $\Gamma_{\mathrm{f}}$ is the partial width for decay by fission and $x$ is the total number of neutrons to be emitted in the formation of the final product [SIK68A]. The cross section for formation of the $\mathrm{CN}$ is given below in the final expression for $\sigma$ :

$$
\sigma=\pi \lambda_{\mathrm{tp}}^{2} \frac{2 \mathrm{I}_{\mathrm{c}}+1}{\left(2 \mathrm{I}_{\mathrm{t}}+1\right)\left(2 \mathrm{I}_{\mathrm{p}}+1\right)} \frac{\Gamma_{\mathrm{tp}} \Gamma}{\left(\mathrm{E}-\mathrm{E}_{\mathrm{o}}\right)^{2}+(\Gamma / 2)^{2}} \mathrm{~W}_{\mathrm{prod}}
$$

where $\lambda_{t p}$ is the reduced DeBroglie wavelength for the entrance channel, $I_{c}, I_{t}$ and $I_{p}$ are the nuclear spins of the $\mathrm{CN}$, target and projectile, respectively, $\mathrm{E}_{\mathrm{o}}$ is the center of mass energy of the target plus projectile system, $\mathrm{E}$ is the excitation energy of the $\mathrm{CN}, \Gamma_{\mathrm{tp}}$ is the partial width for decay into the entrance channel and $\Gamma$ is the total width of the system including all exit channels. The meaning of "decay width" $(\Gamma)$ lies in the fact that $\Gamma_{\mathrm{j}} / \hbar$ is the probability per unit time that the $\mathrm{CN}$ will decay into channel $\mathrm{j}$, where $\mathrm{j}$ could be neutron emission, fission, etc. [FRI81]. In the code, it is assumed that $\Gamma_{\mathrm{n}} / \Gamma_{\mathrm{f}}$ is independent of either the bombarding energy or the excitation energy of the $\mathrm{CN}$ [SIK68A]. Therefore, the shape of the excitation function predicted by SPIT depends mostly on the $\sigma_{\mathrm{CN}}$ term.

$\sigma_{\mathrm{CN}}$ depends on the transmission of the projectile through the potential between the interacting nuclei [SIK68A]. Therefore, the form of the potential energy term, V(r), directly affects the shape of the excitation function predicted by SPIT. SPIT uses a 
potential combining the Bondorf, Sobel and Sperber (BSS) Coulomb potential [BON74] and the Bass proximity potential [BAS74]. The BSS potential has the following form:

$$
\begin{gathered}
V_{B S S}(r)=\frac{Z_{p} Z_{t} e^{2}}{r} \quad \text { for } r \geq R_{p}+R_{t}=R_{c} \\
V_{o}-K r^{n} \quad \text { for } r<R_{c}
\end{gathered}
$$

where $Z_{p}$ and $Z_{t}$ are the atomic numbers of the projectile and target, respectively, e is the electronic charge, $R_{p}, R_{t}$ and $R_{c}$ are the radii of the projectile, target and compound nucleus, respectively and $V_{o}, K$ and $n$ are given by the following equations:

$$
\begin{aligned}
& V_{o}=0.6 e^{2}\left[\frac{\left(Z_{t}+Z_{p}\right)^{2}}{\left(R_{t}^{1 / 3}+R_{p}^{1 / 3}\right)^{3}}-\frac{Z_{t}^{2}}{R_{t}}-\frac{Z_{p}^{2}}{R_{p}}\right] \\
& K=\left(V_{o}-e^{2} Z_{p} Z_{t} / R_{c}\right) / R_{c} \\
& n=\frac{e^{2} Z_{p} Z_{t}}{R_{c}^{2} K}
\end{aligned}
$$

The Bass proximity potential has the form shown in equation 6.11 :

$$
\mathrm{V}_{\text {Bass }}(\mathrm{r})=\mathrm{V}_{\mathrm{BSS}}+\frac{\hbar^{2} \mathrm{~L}^{2}}{2 \mu \mathrm{r}^{2}}-\mathrm{a}_{\mathrm{s}} \mathrm{A}_{\mathrm{p}}^{1 / 3} \mathrm{~A}_{\mathrm{t}}^{1 / 3}\left(\mathrm{~d} / \mathrm{R}_{\mathrm{c}}\right) \exp \left[-\left(\mathrm{r}-\mathrm{R}_{\mathrm{c}}\right) / \mathrm{d}\right]
$$

where $L$ is the total angular momentum of the system, $\mu$ is the reduced mass of the system, $A_{p}$ and $A_{t}$ are the mass numbers of the projectile and target, respectively, $d$ is a range parameter and $a_{s}$ is the surface term in the liquid drop mass formula. Other cross section prediction codes use different forms for the nuclear potential, but the combination of the BSS and Bass formulas may provide a more realistic approximation of the 
internucleus potential, resulting in a better approximation to the shape of the excitation function.

Our research group has used the SPIT code for many years as the first step in evaluating the feasibility of nuclear reactions. The code was developed specifically for compound nuclei that fell within the actinide and transactinide regions of the table of isotopes. It was originally chosen by our research group because fission competition in the $\mathrm{CN}$ was treated explicitly using the empirical $\Gamma_{\mathrm{n}} / \Gamma_{\mathrm{f}}$ formula of Sikkeland et al. [SIK68A]. Other codes did not treat fission competition correctly or simply did not address it. Even though we have found SPIT to be reliable in estimating cross sections, it is still necessary to know how well the code is reproducing experimental data for future evaluations.

\subsubsection{Comparison With Experimental Data}

Throughout Chapter 6, the predictions of the SPIT code have been compared to cross sections calculated from experimental data. We can look at these comparisons in more detail in order to determine the relative accuracy of SPIT for predicting reaction cross sections. Figures $6.17,6.26,6.27,6.28,6.29$ and 6.30 show comparisons of the cross sections measured in this dissertation with the corresponding ones predicted by SPIT.

To begin, we will consider reactions with heavy beams, such as ${ }^{14} \mathrm{~N},{ }^{15} \mathrm{~N}$ and ${ }^{12} \mathrm{C}$. It was discussed in section 6.2.1 that fission data from the ${ }^{233} \mathrm{U}\left({ }^{14} \mathrm{~N}, 5 \mathrm{n}\right){ }^{242}$ Es reaction matched the shape of the excitation function predicted by SPIT (Figure 4.5). The number of coincident fission events from the ECDF of ${ }^{242}$ Es was used as an indication of the 
number of ${ }^{242}$ Es nuclei produced at each projectile energy. The peak energy was also found to be very close to the predicted value of $85 \mathrm{MeV}$. The only possible difference between the code and the data was the shape of the function at lower energies. Figure 4.5 shows a very steep decrease in the predicted production cross sections at lower energies. Our results at $80 \mathrm{MeV}$ and $87 \mathrm{MeV}$ were very close in magnitude, indicating that the low energy part of the function may have a more gradual slope than the one seen in Figure 4.5. Since our results were only based on fission events, we could not determine whether the magnitudes of the predicted cross sections were accurate, but it appeared that SPIT had correctly predicted the shape and peak of the excitation function for the $5 n$ exit channel. Another example of a $5 n$ exit channel reaction was the ${ }^{237} \mathrm{~Np}\left({ }^{12} \mathrm{C}, 5 \mathrm{n}\right){ }^{244} \mathrm{Es}$ reaction used in the study of ECDF in ${ }^{244} \mathrm{Es}$. At $81 \mathrm{MeV}$, the production cross section for this reaction was measured to be $0.31 \pm 0.12 \mathrm{nb}$, compared to the $1.5 \mu \mathrm{b}$ predicted by SPIT. The predicted value was approximately five times larger than the experimental value. It was discussed in section 6.1 that previous experimental data had shown that SPIT was usually correct within a factor of five to the actual cross sections for $5 \mathrm{n}$ exit channel reactions with heavy projectiles. This ${ }^{244}$ Es result seems to further confirm this fact. From the examples presented in section 6.1 and the ${ }^{242}$ Es and ${ }^{244}$ Es results presented here, it appears that SPIT in general has relatively good success in predicting the shape and magnitude of the excitation function for $5 \mathrm{n}$ reactions with heavy projectiles.

Figure 6.17 shows the experimental results and SPIT prediction for the ${ }^{233} \mathrm{U}\left({ }^{14} \mathrm{~N}, 4 \mathrm{n}\right){ }^{243} \mathrm{Es}$ reaction. Both of the experimental cross sections are within a factor of three to the predicted values. The agreement here is actually quite good. The predicted function has a very steep decrease in cross section at low energies. The experimental 
data indicates that there is instead a more gradual decrease in cross section, but more low energy values need to be measured to determine whether the predicted shape of the excitation function is correct. For both the $4 \mathrm{n}$ and $5 \mathrm{n}$ reactions, it seems that SPIT does a decent job of predicting the magnitudes of the production cross sections around the peak energy of the excitation function. SPIT could therefore be used to get an estimate of the peak energy and the relative magnitudes of cross sections around that energy.

SPIT does not do quite as good a job, however, in predicting cross sections for reactions involving light projectiles, such as protons. Figure 6.26 shows the cross sections measured for the ${ }^{249} \mathrm{Cf}(\mathrm{p}, 5 \mathrm{n}){ }^{245}$ Es reaction and the corresponding predictions. At both $35 \mathrm{MeV}$ and $40 \mathrm{MeV}$, SPIT did a terrible job in predicting the magnitude of the cross section for the $5 \mathrm{n}$ reaction. The predictions are approximately two orders of magnitude larger than what was measured experimentally. Unlike the $5 \mathrm{n}$ reactions involving heavy beams, the $5 \mathrm{n}$ reaction with protons was not predicted correctly at all. The shape of the excitation function may have been predicted with relative accuracy, but with only two data points to compare it to, it is difficult to make a definite conclusion.

The results from the ${ }^{249} \mathrm{Cf}(\mathrm{p}, 4 \mathrm{n}){ }^{246}$ Es reaction are shown in Figure 6.27. At lower energies, SPIT did a better job in predicting the magnitude of cross sections than it did for the $5 \mathrm{n}$ exit channel. For instance, the value measured at $28 \mathrm{MeV}$ was only a factor of 10 or so lower than the predicted value. Still, there was not as good agreement as in the reactions with heavy projectiles, but the $4 n$ predictions are much better than those for the $5 \mathrm{n}$ reaction. At higher energies the agreement between experiment and prediction breaks down, and once again the predicted values are almost two orders of magnitude higher than what was measured. Ironically, it would appear that SPIT actually did a relatively 
good job in predicting the shape of the excitation function, as the slopes of the actual . function and the predicted one seem to be nearly identical. Whereas with heavy beams the magnitudes of the cross sections were relatively accurate, with light beams it seems that the shapes are predicted with relative accuracy, but the predicted cross sections are orders of magnitude larger than the actual values.

This trend is also seen in the ${ }^{249} \mathrm{Cf}(\mathrm{p}, 3 \mathrm{n}){ }^{247}$ Es reaction shown in Figure 6.28. At higher projectile energies, the predicted cross sections are actually very close to the experimental ones, within a factor of three. However, agreement is terrible at lower energies, where the predictions are orders of magnitude higher than the actual cross sections. The shape of the excitation function, however, was predicted correctly. Our results for the $3 n$ and $4 n$ reactions show that SPIT predicts the shape of these light projectile excitation functions, but can not predict the absolute magnitudes of the cross sections. In these cases, SPIT could be used for predicting the peak energy of the function, but not the magnitude of the cross section associated with it.

The results for the ${ }^{249} \mathrm{Cf}(\mathrm{p}, 2 \mathrm{n}){ }^{248} \mathrm{Es}$ and ${ }^{249} \mathrm{Cf}(\mathrm{p}, 1 \mathrm{n}){ }^{249} \mathrm{Es}$ reactions are shown in Figures 6.29 and 6.30, respectively. Both figures look very similar, and show that SPIT incorrectly predicted the shape of the excitation function in each case, especially at higher energies. Whereas SPIT predicted a sharply decreasing function with increasing energy, the actual functions appear to have a much more gradual shape, decreasing more slowly with energy. Strangely enough, it would seem that the magnitudes of the predicted cross sections at lower energies are pretty accurate, less than an order of magnitude off from the real values. It appears that SPIT can only predict either the magnitudes of the cross sections or the shape of the excitation function with relative accuracy, but not both 
simultaneously for reactions with light projectiles. For the $1 \mathrm{n}$ and $2 \mathrm{n}$ reactions, the magnitudes were close, but the shapes were incorrect. The opposite effect was seen for the $3 n, 4 n$ and $5 n$ reactions, where the magnitudes could not be predicted by the code, but the shapes of the functions were almost perfect. For light projectile reactions, therefore, SPIT should be avoided for predicting cross sections. One could not be sure whether the code was giving the correct shape or the correct magnitudes. It should, however, be recommended for heavy beams, as the magnitudes were all very close to the actual values. There are other cross section codes that are used at various institutions, and perhaps one of these would be better suited for light beams.

There is a possible explanation as to the differences between the predicted and experimental excitation functions, especially for the $1 \mathrm{n}$ and $2 \mathrm{n}$ light ion reactions. The SPIT code works on the assumption that after the $\mathrm{CN}$ is formed, it attains statistical equilibrium prior to deexcitation [BLA75]. This means that the subsequent decay of the relatively long-lived $\mathrm{CN}$ can be treated by a statistical approach. The shape of an excitation function dominated by this equilibrium assumption would look like the predictions made by the SPIT code shown throughout this dissertation, namely an inverted parabolic function. However, previous excitation function experiments have shown that in some cases, the high energy part of the function does not decrease so sharply, but instead levels off into an almost constant-like function [BLA75]. This high energy phenomenon has been attributed to the emission of particles from the $\mathrm{CN}$ before it reaches statistical equilibrium, otherwise known as preequilibrium decay [BLA75]. In Figures 6.29 and 6.30, it appears that the excitation functions for the $1 \mathrm{n}$ and $2 \mathrm{n}$ exit channels might be showing this preequilibrium decay, as the functions appear to level off 
at higher energies. Looking at Figure 6.28 , it seems that the $3 \mathrm{n}$ reaction might also be experiencing preequilibirum decay, as the function appears to start leveling off at higher energies. Since the model used by the SPIT code does not take this form of deexcitation into account, it might explain why the predicted shapes of the functions for the $1 \mathrm{n}$ and $2 \mathrm{n}$ reactions were so different than what was seen experimentally. For a more detailed discussion of preequilibrium decay, please see [BLA75] and the references therein.

\subsubsection{Another Example of a Cross Section Code}

There are several other cross section prediction codes in circulation at other laboratories and universities. While our group has predominantly used the SPIT code for predicting cross sections, in order to truly evaluate its performance we need to look into other codes and see how well they predict the magnitudes and shapes of excitation functions. It seems that each code is suited to a particular region of the chart of the nuclides or to a specific type of reaction, making it difficult to decide which code would be best for our purposes. It would be useful to find a code that was better for predicting cross sections from reactions using light beams. One possibility for this is the ALICE code [BLA82], which is in circulation at Lawrence Livermore National Laboratory. ALICE does include a factor that takes preequilibrium decay into account, so the shapes of the excitation functions, especially for reactions involving light projectiles, may be predicted with more accuracy than SPIT. The code uses a Weisskopf-Ewing evaporation calculation [WEI40], the Bohr-Wheeler transition state model for fission [BOH39] and the hybrid/geometry dependent hybrid model for preequilibrium decay [BLA71, BLA72]. 
For more information about these calculations, the reader is referred to the references given here.

One feature of the ALICE code is that the user must enter several variables that are normally treated as constants in the SPIT code. What this means is that one must find experimental cross section data for the region of interest, and then find the correct variables that enable ALICE to replicate these values. Once the variables have been determined, the code can then be used for unknown systems, but only for that particular region. If one tries to use the same variables for all reactions, there is no consistent replication of experimental cross sections. This limits the code's usefulness, as it becomes specific only to one particular region of nuclides. In this sense, SPIT is more useful, as it covers a much broader range of nuclear reactions. To be certain, no one code is perfect for all situations, but perhaps a combination of different codes would sufficiently cover most reactions used for isotope production.

In the ALICE code the user must set a variable that scales the fission barrier. By varying this parameter, it was found that a setting between 2.0 and 3.0 worked best for einsteinium compound nuclei. The first reactions investigated using ALICE were the ${ }^{233} \mathrm{U}\left({ }^{14} \mathrm{~N}, \mathrm{xn}\right){ }^{247-\mathrm{x}} \mathrm{Es}$ reactions at $80 \mathrm{MeV}$ and $87 \mathrm{MeV}$. Figure 6.17 shows the experimentally determined $4 \mathrm{n}$ cross sections and the corresponding SPIT predictions. The ALICE predictions were closest to the experimental values when a fission barrier scaling factor of 2.6 was used. At a projectile energy of $80 \mathrm{MeV}$, the ${ }^{243}$ Es cross section was predicted to be $49 \mathrm{nb}$, which is in good agreement with the experimental value of 33 $\pm 17 \mathrm{nb}$. However, the $87 \mathrm{MeV}$ prediction was only $1.6 \mathrm{nb}$, over an order of magnitude smaller than the experimentally determined value of $48 \pm 25 \mathrm{nb}$. When different fission 
barrier scaling factors were tried, the cross section at $87 \mathrm{MeV}$ was always an order of magnitude less than the value at $80 \mathrm{MeV}$. SPIT predictions and the experimental values were the same order of magnitude at both projectile energies. For these reactions, it appears that SPIT does a better job of predicting the magnitudes of the cross sections than ALICE does.

A scaling factor of 2.5 was then used for the ${ }^{233} \mathrm{U}\left({ }^{15} \mathrm{~N}, \mathrm{xn}\right)^{248-\mathrm{x}} \mathrm{Es}$ reactions at projectile energies of $80 \mathrm{MeV}$ and $86 \mathrm{MeV}$. At $80 \mathrm{MeV}$, the predicted cross section for the $3 \mathrm{n}$ reaction was $5.3 \mathrm{nb}$, which does not agree at all with the $60 \pm 23-\mathrm{nb}$ cross section measured experimentally for ${ }^{245}$ Es. However, the code did a little better at $86 \mathrm{MeV}$, where it predicted a cross section of $88 \mathrm{nb}$ for ${ }^{244} \mathrm{Es}$, which is actually quite close to the experimental value of $73 \pm 54 \mathrm{nb}$. Once again, the code was able to predict one of the cross sections correctly, but was over an order of magnitude smaller for the other. Without any experimental data to compare the predictions to, it would be very difficult to use ALICE to predict cross sections for this type of reaction, because it would be impossible to tell whether the code was predicting the correct cross section or was in fact an order of magnitude off. Previous data have shown that for reactions involving heavy beams, the SPIT code is a better choice for predicting the magnitudes of cross sections.

Finally, the ${ }^{249} \mathrm{Cf}(\mathrm{p}, \mathrm{xn})^{250-\mathrm{x}}$ Es reactions were investigated using a scaling factor of 2.4. Figures 6.26 through 6.30 show the experimentally determined cross sections as well as the corresponding SPIT predictions, and Table 7.2 lists all of the cross sections measured as part of this dissertation, along with the SPIT and ALICE predictions for each case. The reader is referred to that table for the specific ALICE predictions for these isotopes, but some general trends of the ability of the ALICE code to predict data will be 
mentioned here. For ${ }^{245}$ Es, the predicted cross sections are both too large by at least an order of magnitude. At lower energies, the cross sections for ${ }^{246}$ Es are actually predicted correctly within a factor of two. But at proton energies of $30 \mathrm{MeV}$ or greater, the agreement really breaks down and the predicted ${ }^{246}$ Es cross sections are one or more orders of magnitude too large. The ALICE values for ${ }^{247}$ Es have better success, as the cross sections agree relatively well with the experimental values. Decent agreement between ALICE predictions and experiment was also observed for ${ }^{248}$ Es and ${ }^{249}$ Es. ALICE apparently does a good job of predicting cross sections for the heavier einsteinium isotopes, but breaks down when predicting cross sections for the lighter nuclides. It is not clear whether the predicted values begin to disagree with experiment after the evaporation of a certain number of neutrons, or whether a different scaling factor needs to be used for the heavier nuclides. In general, ALICE does do a better job in predicting cross sections for reactions involving light beams, but one must know the correct scaling factor to use ahead of time. If the wrong scaling factor is used, the predicted cross sections are incorrect by several orders of magnitude. This limits the usefulness of the code. If one were to use ALICE to predict relative cross sections without any a priori knowledge of what scaling factor to use, the user would undoubtedly be given the wrong information. In general, ALICE could be used for light beams, but only if the scaling factor could be determined from previously reported data. For heavy beams, SPIT is by far the better choice. 


\section{Summary}

\subsection{ECDF of ${ }^{242} \mathrm{Es}$}

${ }^{242}$ Es was produced via the ${ }^{233} \mathrm{U}\left({ }^{14} \mathrm{~N}, 5 \mathrm{n}\right){ }^{242}$ Es reaction using a single target with projectile energies of $84 \mathrm{MeV}, 87 \mathrm{MeV}$ and $89 \mathrm{MeV}$ entering the target. A total of 48 coincident fission pairs were detected over the course of three experiments. In the ECDF process the fission is very fast compared to the initial EC-decay so the fission events decay with the half-life of the EC-parent. Based on the decay of the 48 fission events, a half-life of $11 \pm 3 \mathrm{~s}$ was determined for ${ }^{242} \mathrm{Es}$. Our value for the half-life is consistent with the previous value of $16_{-4}^{+6} \mathrm{~s}$ reported by Ninov et al. [NIN96], but our value is based on a larger number of ${ }^{242}$ Es events so the statistical error is smaller. This half-life confirms the identity of the EC-precursor as ${ }^{242}$ Es since there are no spontaneous fission isotopes in this region that could have contributed to the number of fission events detected. Analysis of the kinetic energies of the coincident fission events showed a highly asymmetric mass distribution, with mass peaks at 104 and 138 . The average and most probable pre-neutron emission TKE values were $183 \pm 18 \mathrm{MeV}$ and $182 \pm 20 \mathrm{MeV}$, respectively. From comparison with the static fission model of Wilkins et al. [WIL76] and systematics, the heavy fragment is probably nearly spherical while the light fragment is highly deformed. The $\mathrm{P}_{\mathrm{DF}}$ was measured to be $0.006 \pm 0.002$ and is the largest $\mathrm{P}_{\mathrm{DF}}$ ever measured, which is expected based on the larger $\mathrm{QEC}_{\mathrm{EC}}$ of ${ }^{242} \mathrm{Es}$.

A lower limit of $22 \mathrm{nb}$ was measured for the production cross section of the ${ }^{233} \mathrm{U}\left({ }^{14} \mathrm{~N}, 5 \mathrm{n}\right){ }^{242}$ Es reaction at $87 \mathrm{MeV}$ assuming a $100 \%$ EC-branch in ${ }^{242}$ Es. We also measured an upper limit of 0.25 for the $\alpha$-branch and a lower limit of 0.75 for the EC- 
branch of ${ }^{242}$ Es. Using the single-particle Nilsson diagrams [FIR96] with a predicted nuclear deformation $(\beta)$ of 0.208 for ${ }^{242}$ Es [MÖL95], we were able to conclude that the ground state to ground state ${ }^{242} \mathrm{Es}$ to ${ }^{242} \mathrm{Cf} \mathrm{EC}$-transition is most likely an allowed transition. Assuming that our measured cross section is within a factor of five to the actual value and using our measured limit of the EC-branch, we can reason that the log $f t$ value falls between 4.5 and 4.7 [FIR96], indicative of an allowed transition. If the ECbranch of ${ }^{242} E s$ is measured at some future time, the production cross section and $\log f t$ can be more accurately determined.

\subsection{ECDF of ${ }^{244} \mathrm{Es}$}

${ }^{244}$ Es was produced via the ${ }^{233} \mathrm{U}\left({ }^{15} \mathrm{~N}, 4 \mathrm{n}\right){ }^{244}$ Es reaction using a single target at energies of $81 \mathrm{MeV}$ and $86 \mathrm{MeV}$ at the start of the target. Only two coincident fission events were detected over the course of the experiment. Based on the $\alpha$-decay of ${ }^{244} \mathrm{Es}$, a production cross section of $73 \pm 54 \mathrm{nb}$ was determined for the ${ }^{233} \mathrm{U}\left({ }^{15} \mathrm{~N}, 4 \mathrm{n}\right){ }^{244}$ Es reaction at $86 \mathrm{MeV}$, which was much lower than we originally expected, and accounts for the low number of fission events we detected.

${ }^{244} \mathrm{Es}$ was then produced using the ${ }^{237} \mathrm{~Np}\left({ }^{12} \mathrm{C}, 5 \mathrm{n}\right){ }^{244} \mathrm{Es}$ reaction with a single target at $81 \mathrm{MeV}$. A total of 16 coincident fission events were detected over the course of the experiment. The fission events had a half-life of $31 \pm 10 \mathrm{~s}$, close to the previously reported value of $37 \mathrm{~s}$ for ${ }^{244} \mathrm{Es}$. This half-life confirms the identity of the EC-parent as ${ }^{244}$ Es. There are no spontaneous fission isotopes in this region that could have contributed to the number of events detected. Analysis of the kinetic energies of the coincident fission events showed a highly asymmetric mass distribution with mass peaks at 103 and 
141. The average and most probable pre-neutron emission TKE values were $186 \pm 19$ $\mathrm{MeV}$ and $193 \pm 20 \mathrm{MeV}$, respectively. The difference between the average and most probable TKE values is due to poor sample quality from thick $\mathrm{KCl}$ deposits on the foils, resulting in degradation of fission fragment energy. From the static fission model of Wilkins et al. [WIL76] the heavy fragment is most likely nearly spherical and the light fragment is highly deformed. The $P_{D F}$ was measured to be $(1.2 \pm 0.4) \times 10^{-4}$. A production cross section of $0.31 \pm 0.12 \mu \mathrm{b}$ was calculated for the ${ }^{237} \mathrm{~Np}\left({ }^{12} \mathrm{C}, 5 \mathrm{n}\right){ }^{244} \mathrm{Es}$ reaction at $81 \mathrm{MeV}$. Using the single particle Nilsson diagrams in [FIR96] and a predicted $\beta$ value of 0.217 for ${ }^{244} E s$ [MÖL95], a $\log f t$ of 6.2 was calculated for the ground state to ground state ${ }^{244} \mathrm{Es}$ to ${ }^{244} \mathrm{Cf}$ EC-transition, which is indicative of a firstforbidden transition.

\subsection{Fission Properties From ECDF Studies}

Figure 7.1 shows the average or most probable TKE values versus $Z^{2} / A^{1 / 3}$ for all known cases of spontaneous or delayed fission, including the average values for ${ }^{242} \mathrm{Cf}$ and ${ }^{244} \mathrm{Cf}$, along with the empirical fits of Viola et al. [VIO85] and Unik et al. [UNI73]. $\mathrm{Z}^{2} / \mathrm{A}^{1 / 3}$ comes from the Coulomb repulsion term in the liquid drop mass formula [KRA88], and is a measure of the tendency of a nucleus to break apart due to the Coulomb repulsion from the protons. The errors are not plotted in Figure 7.1 for clarity. Although it appears that the delayed fission cases consistently have lower TKE values than the spontaneously fissioning isotopes, the errors on the values for ${ }^{242} \mathrm{Cf}$ and ${ }^{244} \mathrm{Cf}$ are so large that it leaves some uncertainty as to whether these nuclides are following the trend observed in earlier ECDF studies. However, the errors associated with the 


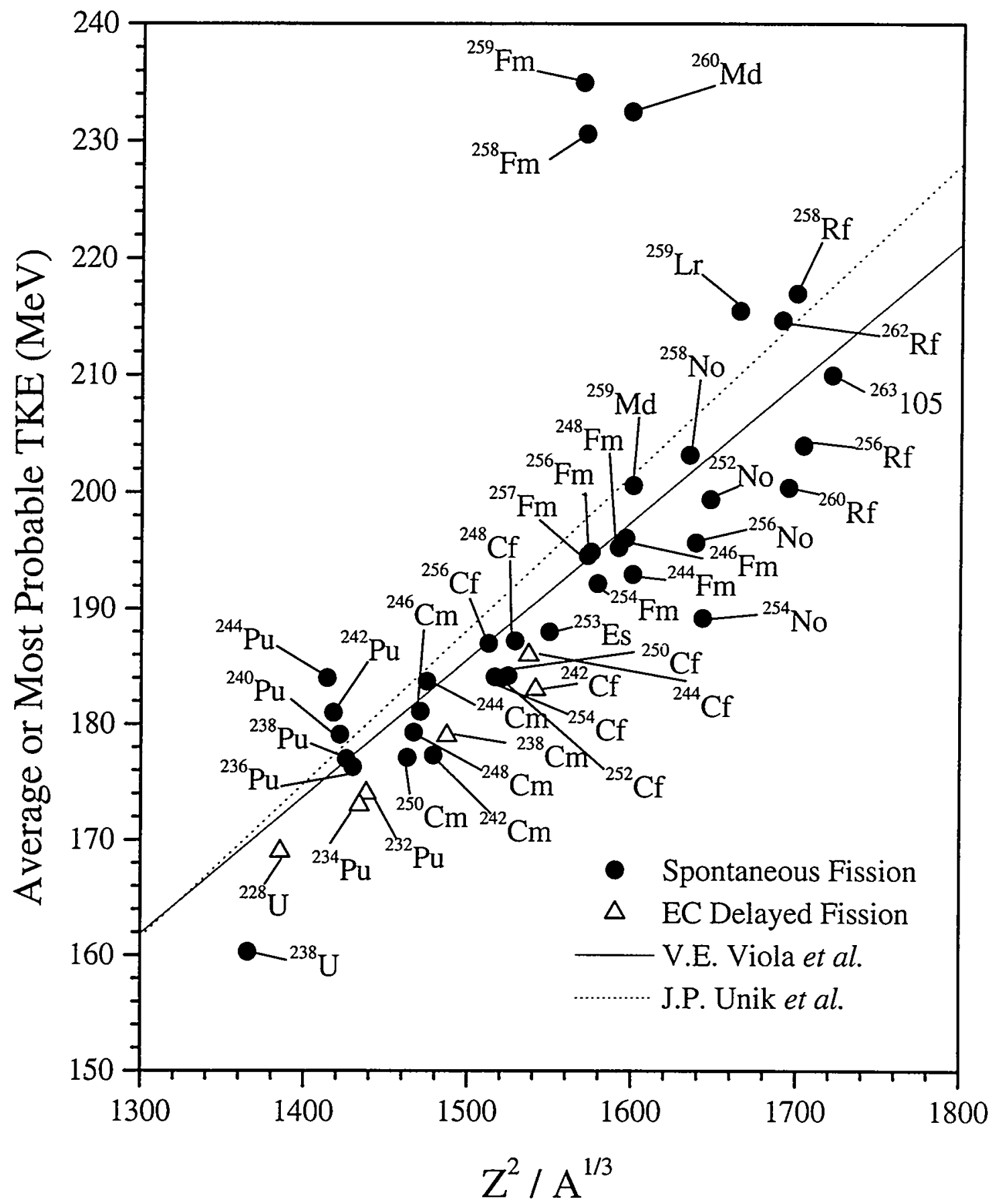

Figure 7.1: The average or most probable TKE vs $Z^{2} / A^{1 / 3}$ for known cases of spontaneous or delayed fission [LAN98]. The solid line is the linear fit of Viola et al. [VIO85] and the dashed line is from Unik et al. [UNI73]. All of the average TKE values have been corrected to be consistent with the calibration parameters of Weissenberger et al. [WEI86]. In the case of delayed fission the $\mathrm{Z}^{2} / \mathrm{A}^{1 / 3}$ of the fissioning daughter is used. 
previously reported TKE values from ECDF experiments are relatively small, approximately $5 \mathrm{MeV}$ [KRE94, KRE94A, HAL90, HAL90A]. Even though our statistical errors are large, the smaller errors of the previous data points imply that the trend of lower TKE values in ECDF is consistent and continues through the einsteinium region. It would be beneficial to make further measurements of the average TKE values of ${ }^{242} \mathrm{Cf}$ and ${ }^{244} \mathrm{Cf}$ in the future to lower the statistical errors of these values and confirm that this trend in TKE applies to these nuclides as well. This trend, if it can be confirmed, is due to the extra excitation energy inherent in the delayed fission process: ECDF can impart excitation energy to the fissioning species up to the entire $\mathrm{Q}_{\mathrm{EC}}$ [KRE93]. This means that up to $5 \mathrm{MeV}$ of energy can be imparted to the EC-daughter before it undergoes fission. Excitation energy tends to wash out shell effects in fission fragments, which are normally very strong in the case of spontaneous fission. In delayed fission the shell effects would be weaker causing the heavy fragments located around the $\mathrm{N}=82$ spherical shell to be more deformed than they would be in the case of spontaneous fission, where there is either one spherical fragment and one highly deformed fragment or two nearly spherical fragments as for ${ }^{258,259} \mathrm{Fm}$ and ${ }^{260} \mathrm{Md}$. Weaker shell effects would cause one slightly deformed fragment and one highly deformed fragment in ECDF, resulting in a lower overall TKE than if the heavy fragment were more spherical.

\subsection{ECDF of ${ }^{246}$ Es and ${ }^{248} \mathrm{Es}$}

${ }^{246} \mathrm{Es}$ and ${ }^{248} \mathrm{Es}$ were both produced via the ${ }^{249} \mathrm{Cf}(\mathrm{p}, \mathrm{xn})^{250-\mathrm{x}}$ Es reaction at projectile energies of 37 and $18 \mathrm{MeV}$, respectively. Nineteen ${ }^{249} \mathrm{Cf}$ targets were bombarded simultaneously in the LIM target system. At $37 \mathrm{MeV}$, one coincident fission event was 
detected. This was not a background event because background measurements taken before the experiment showed a background fission rate of less than one per day. The event was attributed to ${ }^{246}$ Es since no evidence of ${ }^{248}$ Es (the only other ECDF isotope produced in this reaction) was observed in the $\alpha$-spectra. Based on an initial ${ }^{246}$ Es $\alpha$ activity of 654 counts/min and the known EC-branch of $90 \%$, a $P_{D F}$ of $(3.7 \pm 3.7) \times 10^{-5}$ was calculated for ${ }^{246}$ Es. This value really represents an upper limit of the $P_{D F}$ because it is still possible that the one fission event observed could be attributed to background, even though background measurements indicated otherwise. Using the single particle Nilsson diagrams in [FIR96] and a predicted $\beta$ of 0.217 for ${ }^{246}$ Es [MÖL95], a $\log f t$ of 5.9 was determined for the ground state to ground state ${ }^{246} \mathrm{Es}$ to ${ }^{246} \mathrm{Cf}$ EC-transition. This transition is an isospin forbidden transition, which typically has a $\log f t$ of 6.5 or greater. Either the $\beta$ predicted for ${ }^{246} \mathrm{Es}$ is incorrect, or this particular transition is faster than other isospin forbidden transitions.

Four coincident fission events were detected at a projectile energy of $18 \mathrm{MeV}$. No ${ }^{246}$ Es was produced at this energy, so the fission events must have come from the ECDF of ${ }^{248}$ Es. Based on an initial ${ }^{248}$ Es $\alpha$-activity of 423 counts/min and its known EC branch of $99.7 \%$, a $P_{D F}$ of $(3.5 \pm 1.8) \times 10^{-6}$ was determined for ${ }^{248}$ Es. Based on the single particle Nilsson diagrams [FIR96] and a predicted $\beta$ of 0.217 [MÖL95], the $\log f t$ of the ground state to ground state ${ }^{248} \mathrm{Es}$ to ${ }^{248} \mathrm{Cf}$ EC-transition is 6.1 , indicative of a firstforbidden transition. At both beam energies, we had expected to see thousands of fission events, assuming a gas-jet yield of approximately $50 \%$. Since the transport efficiency was the only unknown, the low number of fission events meant that the yield was actually 
much less than the previously reported value of $50 \%$ and subsequent tests of the LIM system were performed.

\section{5 $\mathrm{P}_{\mathrm{DF}}$ and $\log f t$ Values}

Figure 7.2 shows all of the $P_{D F}$ values measured by our research group versus $\mathrm{Q}_{\mathrm{EC}}$. In Chapter 2 it was shown that the $\mathrm{P}_{\mathrm{DF}}$ is directly influenced by the $\mathrm{Q}_{\mathrm{EC}}$, and the probability should increase with increasing Q-value. This is confirmed in Figure 7.2. There appears to be an exponential relationship between the $P_{D F}$ and $Q_{E C}$. This strong dependence indicates that the $\mathrm{Q}_{\mathrm{EC}}$ is the major parameter influencing the magnitude of the $\mathrm{P}_{\mathrm{DF}}$. The other factor that would influence the $\mathrm{P}_{\mathrm{DF}}$ is the fission barrier height. It has been shown, however, that the heights of the fission barriers do not vary greatly with neutron number for nuclides in the region where ECDF occurs [BRI80]. Therefore, the $\mathrm{P}_{\mathrm{DF}}$ must have a large dependence on the $\mathrm{Q}_{\mathrm{EC}}$, since the fission barriers do not change enough to account for the large exponential dependence observed in Figure 7.2. Also, it was noted that our value for the $\mathrm{P}_{\mathrm{DF}}$ of ${ }^{248} \mathrm{Es}$ was an order of magnitude larger than the previously reported value of $3 \times 10^{-7}$ by Gangrskii et al. [GAN80]. As discussed in Chapter 3, Gangrskii et al. did not include any errors on their reported $\mathrm{P}_{\mathrm{DF}}$ values. Also, they measured fission fragments with a separate detection system than the one that was used to measure $\alpha$-particles of the californium EC-daughters. Each method of detection has errors associated with it that must be included in the final $\mathrm{P}_{\mathrm{DF}}$ value since fission events and $\alpha$-particles were not measured simultaneously. Since Gangrskii et al. did not include any kind of relative error on their value for the $\mathrm{P}_{\mathrm{DF}}$ of ${ }^{248} \mathrm{Es}$, it is impossible to compare our value with theirs in any meaningful way. 


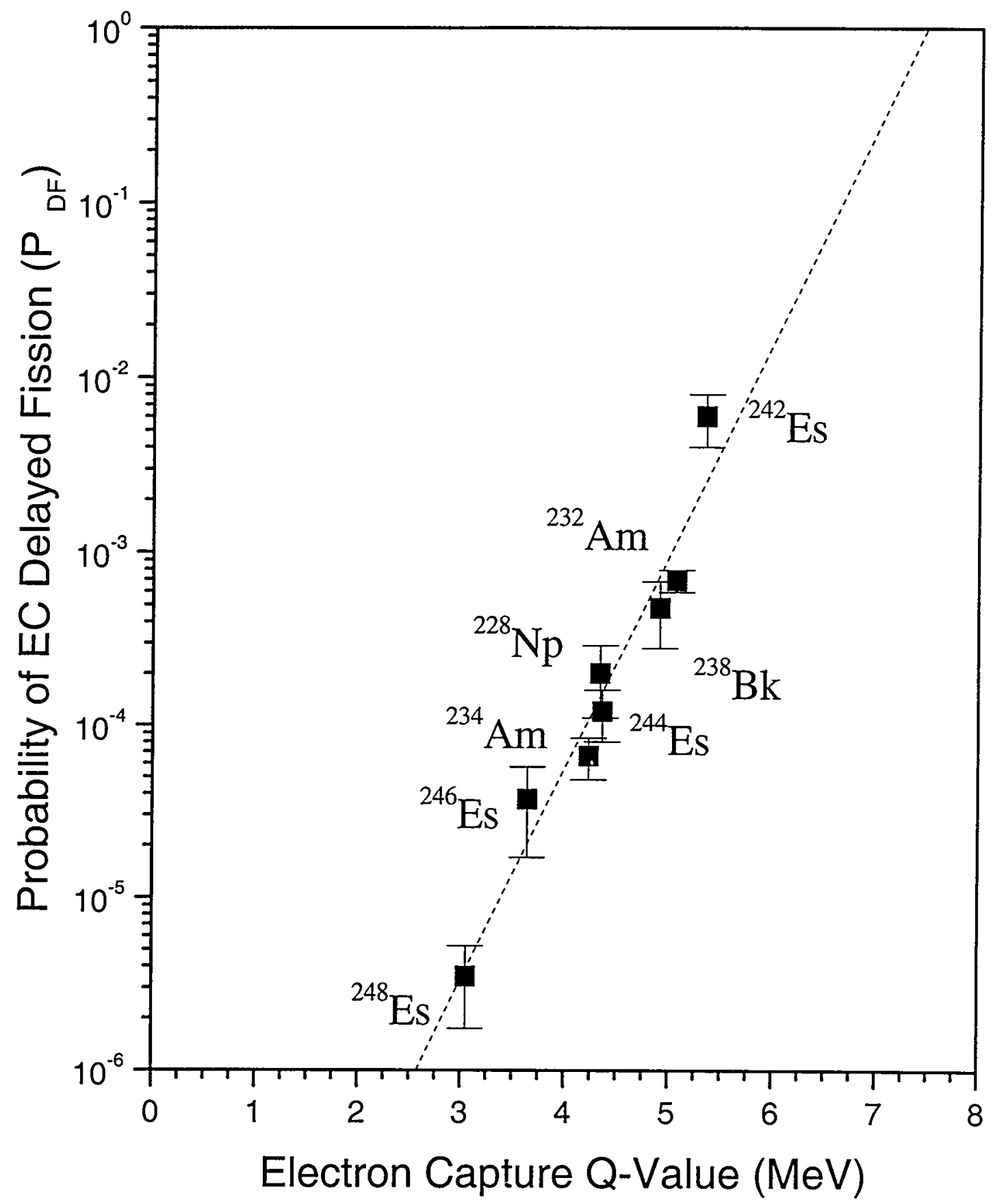

Figure 7.2: Plot of the probability of delayed fission versus $\mathrm{Q}_{\mathrm{EC}}$ for nuclides studied by our research group. The values for ${ }^{232} \mathrm{Am}$ and ${ }^{234} \mathrm{Am}$ are from [HAL90, HAL90A] ${ }^{228} \mathrm{~Np}$ is from [KRE94A] and ${ }^{238} \mathrm{Bk}$ is from [KRE94]. The value for ${ }^{246} \mathrm{Es}$ could be interpreted as an upper limit based on considerations given in the text. 
Table 7.1 gives a summary of the single particle levels and $\log f t$ values that were calculated in this dissertation. The proton and neutron level assignments were all determined using the single particle Nilsson diagrams [FIR96] with predicted nuclear deformations ( $\beta$ ) given by Möller, et al. [MÖL95]. All of the $\log f t$ values quoted in this dissertation were calculated assuming that the EC-transitions were from the ground state of the parent to the ground state of the daughter. If an EC-decay were to populate an excited state in the daughter, then the $\log f t$ values presented here would not apply to the given transition.

Table 7.1. Summary of single particle level assignments and calculated $\log f t$ values assuming ground state to ground state EC-transitions.

\begin{tabular}{|cccccccc|}
\hline Nuclide & $\beta^{\mathrm{a}}$ & $\begin{array}{c}\text { Proton } \\
\text { assignment }\end{array}$ & $\begin{array}{c}\text { Neutron } \\
\text { assignment }\end{array}$ & $\begin{array}{c}\text { Nuclear } \\
\text { spin and } \\
\text { parity }\end{array}$ & $\begin{array}{c}\Delta \mathrm{J}^{\pi} \text { of } \\
\text { transition }\end{array}$ & $\begin{array}{c}\text { Transition } \\
\text { type }\end{array}$ & $\log f t$ \\
\hline${ }^{242}$ Es & 0.208 & $7 / 2^{+}[633]$ & $5 / 2^{+}[622]$ & $1^{+}$ & $1^{\text {no }}$ & Allowed & $\geq 4.5$ \\
\hline${ }^{244}$ Es & 0.217 & $7 / 2^{+}[633]$ & $7 / 2[743]$ & $0^{-}$ & $0^{\text {yes }}$ & $\begin{array}{c}\text { First- } \\
\text { forbidden }\end{array}$ & 6.2 \\
\hline${ }^{246}$ Es & 0.217 & $7 / 2^{+}[633]$ & $7 / 2^{+}[624]$ & $0^{+}$ & $0^{\text {no }}$ & $\begin{array}{c}\text { Isospin } \\
\text { forbidden }\end{array}$ & 5.9 \\
\hline${ }^{248}$ Es & 0.217 & $7 / 2^{+}[633]$ & $9 / 2[734]$ & $1^{-}$ & $1^{\text {yes }}$ & $\begin{array}{c}\text { First- } \\
\text { forbidden }\end{array}$ & 6.1 \\
\hline
\end{tabular}

apredicted nuclear deformation values are from [MÖL95].

\subsection{Excitation Functions}

In the ${ }^{246}$ Es and ${ }^{248}$ Es ECDF experiments we had originally expected to see thousands of fission events per day, but instead only saw a few events over the course of the whole experiment. The only uncertainty was the yield of the LIM system. During the subsequent test of the LIM transport system, $11{ }^{233} \mathrm{U}$ targets were irradiated with $50-\mathrm{MeV}$ protons to make ${ }^{229} \mathrm{~Np}$. By changing the target configurations and measuring the $\alpha$ particle rate, it was noted that not all 11 targets were contributing to the overall yield of 
the system. On going from one target to $1 !$ targets, the $\alpha$-rate only increased by a factor of six instead of the factor of 11 we has expected based on previous results. It was thought that either the $\mathrm{KCl}$ aerosols were depositing on the target holders instead of entering the gas-jet, or thermal motion was causing the reaction products to come back into contact with the target material before they had a chance to attach themselves to $\mathrm{KCl}$ aerosols. The latter effect would be due to the small amounts of recoil energy imparted to the compound nuclei by the proton beam, resulting in very small recoil ranges of the reaction products. If the recoil range is too small, the reaction products will not make it into the stream of the gas-jet, and could instead move back toward the target. Further evidence of this latter effect was observed later.

A gold catcher foil was then placed behind one ${ }^{249} \mathrm{Cf}$ target, which was irradiated with $21-\mathrm{MeV}$ protons. Based on the amounts of ${ }^{248}$ Es and ${ }^{249}$ Es measured in the catcher foil, and by comparing these numbers to what was observed during the excitation function experiment, an overall LIM transport efficiency of only 5-15\% was measured. There seemed to be a slight variation of yield with half-life, indicating that the amount of time needed for reaction products to diffuse out of the target toward the gas-jet was very long. There was also a variation in yield with beam energy. At higher energies, the LIM system had a yield closer to $50 \%$. More energy is imparted to the compound nucleus when the projectile energy is larger, resulting in a larger recoil range. The reaction products, therefore, have a better chance of recoiling out of the target and making it to the gas-jet, increasing the efficiency of the system. At lower beam energies, the recoil range is too low for the reaction products to make it out of the target, and nuclides must rely on thermal motion to bring them out of the target material and into contact with $\mathrm{KCl}$ 
aerosols. That is probably why longer-lived ${ }^{249}$ Es seemed to have a slightly higher yield than ${ }^{248}$ Es. Neither nuclide had a large enough recoil range to recoil out of the targets, but because ${ }^{249}$ Es was longer-lived, over time, more of its nuclei had a chance to diffuse out, increasing its yield over that of ${ }^{248} \mathrm{Es}$. These results do not contradict the previously reported LIM yields of 50\%-95\% of Hall [HAL89]. Those experiments typically used $\alpha$ beams at higher energies than the experiments discussed in this dissertation, resulting in larger reaction product yields than we measured. Our results add to our overall understanding of how the LIM system works.

Excitation function experiments were performed for the ${ }^{233} \mathrm{U}\left({ }^{14} \mathrm{~N}, \mathrm{xn}\right)^{247-\mathrm{x}} \mathrm{Es}$, ${ }^{233} \mathrm{U}\left({ }^{15} \mathrm{~N}, \mathrm{xn}\right){ }^{248-\mathrm{x}} \mathrm{Es}$ and ${ }^{249} \mathrm{Cf}(\mathrm{p}, \mathrm{xn}){ }^{250-\mathrm{x}}$ Es reactions. The ${ }^{14} \mathrm{~N}$ projectiles were at energies of $80 \mathrm{MeV}, 87 \mathrm{MeV}, 93 \mathrm{MeV}$ and $100 \mathrm{MeV}$ (on target), and the ${ }^{15} \mathrm{~N}$ beams were at energies of $80 \mathrm{MeV}, 85 \mathrm{MeV}, 93 \mathrm{MeV}$ and $100 \mathrm{MeV}$ (on target.) Fission data from the experiment using ${ }^{14} \mathrm{~N}$ projectiles showed that the amount of ${ }^{242}$ Es produced went in the following order: $87 \mathrm{MeV}>80 \mathrm{MeV}>93 \mathrm{MeV}>100 \mathrm{MeV}$. These results matched the predictions of the SPIT [SIK67] cross section prediction code. The cross sections calculated from these reactions are given in Table 7.2.

The ${ }^{249} \mathrm{Cf}(\mathrm{p}, \mathrm{xn})^{250-\mathrm{x}} \mathrm{Es}$ reactions were investigated at $21 \mathrm{MeV}, 28 \mathrm{MeV}, 35 \mathrm{MeV}$ and $40 \mathrm{MeV}$ (on target). Several different einsteinium isotopes were produced at each energy, and a combination of on-line counting and chemical separations was used to measure their production cross sections. Based on the tests of the LIM transport system, a gas-jet efficiency of $50 \pm 40 \%$ was used with proton energies of $35 \mathrm{MeV}$ and $40 \mathrm{MeV}$, 
Table 7.2. Experimentally determined cross sections for the production of various einsteinium isotopes.

\begin{tabular}{|c|c|c|c|c|c|}
\hline Nuclide & $\begin{array}{c}\text { Production } \\
\text { reaction }\end{array}$ & $\begin{array}{c}\text { Beam energy } \\
\text { on target } \\
(\mathrm{MeV})\end{array}$ & Cross section & $\begin{array}{c}\text { SPIT } \\
\text { prediction }\end{array}$ & $\begin{array}{c}\text { ALICE } \\
\text { prediction }^{b}\end{array}$ \\
\hline$\overline{{ }^{242} \mathrm{Es}}$ & ${ }^{233} U\left({ }^{14} N, 5 n\right){ }^{242} E s$ & $87 \pm 0.5^{c}$ & $\geq 22 n b^{a}$ & $32 \mathrm{nb}$ & $0.20 \mu \mathrm{b}$ \\
\hline${ }^{243} \mathrm{Es}$ & ${ }^{233} \mathrm{U}\left({ }^{14} \mathrm{~N}, 4 \mathrm{n}\right){ }^{243} \mathrm{Es}$ & $80 \pm 0.5^{c}$ & $33 \pm 17 \mathrm{nb}$ & $73 \mathrm{nb}$ & $49 \mathrm{nb}$ \\
\hline${ }^{243}$ Es & ${ }^{233} \mathrm{U}\left({ }^{14} \mathrm{~N}, 4 \mathrm{n}\right){ }^{243} \mathrm{Es}$ & $87 \pm 0.5^{c}$ & $48 \pm 25 \mathrm{nb}$ & $19 \mathrm{nb}$ & $1.6 \mathrm{nb}$ \\
\hline${ }^{243} \mathrm{Es}$ & ${ }^{233} U\left({ }^{14} \mathrm{~N}, 4 \mathrm{n}\right){ }^{243} \mathrm{Es}$ & $93 \pm 0.5^{c}$ & $\leq 20 \mathrm{nb}^{\mathrm{d}}$ & $2.8 \mathrm{nb}$ & $0.05 \mathrm{nb}$ \\
\hline${ }^{243} \mathrm{Es}$ & ${ }^{233} \mathrm{U}\left({ }^{14} \mathrm{~N}, 4 \mathrm{n}\right){ }^{243} \mathrm{Es}$ & $100.0 \pm 0.5^{c}$ & $\leq 15 \mathrm{nb}^{\mathrm{d}}$ & $0.10 \mathrm{nb}$ & $0.001 \mathrm{nb}$ \\
\hline${ }^{244} \mathrm{Es}$ & ${ }^{233} \mathrm{U}\left({ }^{15} \mathrm{~N}, 4 \mathrm{n}\right){ }^{244} \mathrm{Es}$ & $86 \pm 0.5^{c}$ & $73 \pm 54 \mathrm{nb}$ & $0.65 \mu \mathrm{b}$ & $88 \mathrm{nb}$ \\
\hline${ }^{244}$ Es & ${ }^{237} \mathrm{~Np}\left({ }^{12} \mathrm{C}, 5 n\right){ }^{244} \mathrm{Es}$ & $81 \pm 0.5^{c}$ & $0.31 \pm 0.12 \mu \mathrm{b}$ & $1.6 \mu b$ & $0.52 \mu \mathrm{b}$ \\
\hline${ }^{245} \mathrm{Es}$ & ${ }^{233} \mathrm{U}\left({ }^{15} \mathrm{~N}, 3 \mathrm{n}\right){ }^{245} \mathrm{Es}$ & $80 \pm 0.5^{c}$ & $60 \pm 23 \mathrm{nb}$ & $0.22 \mu b$ & $2.7 \mathrm{nb}$ \\
\hline${ }^{245} \mathrm{Es}$ & ${ }^{249} \mathrm{Cf}(\mathrm{p}, 5 \mathrm{n}){ }^{245} \mathrm{Es}$ & 35 & $0.05 \pm 0.04 \mu \mathrm{b}$ & $3 \mu b$ & $0.57 \mu \mathrm{b}$ \\
\hline${ }^{245}$ Es & ${ }^{249} \mathrm{Cf}(\mathrm{p}, 5 \mathrm{n}){ }^{245}$ Es & 40 & $0.3 \pm 0.2 \mu \mathrm{b}$ & $30 \mu \mathrm{b}$ & $16 \mu \mathrm{b}$ \\
\hline${ }^{246} \mathrm{Es}$ & ${ }^{249} \mathrm{Cf}(\mathrm{p}, 4 n){ }^{246} \mathrm{Es}$ & $21 \pm 1.4$ & $2 \pm 1 \mu b$ & 0 & 0 \\
\hline${ }^{246}$ Es & ${ }^{249} \mathrm{Cf}(\mathrm{p}, 4 \mathrm{n}){ }^{246} \mathrm{Es}$ & 28 & $10 \pm 8 \mu \mathrm{b}$ & $0.20 \mathrm{mb}$ & $26 \mu \mathrm{b}$ \\
\hline${ }^{246} \mathrm{Es}$ & ${ }^{249} \mathrm{Cf}(\mathrm{p}, 4 \mathrm{n}){ }^{246} \mathrm{Es}$ & 35 & $9 \pm 6 \mu \mathrm{b}$ & $0.47 \mathrm{mb}$ & $0.14 \mathrm{mb}$ \\
\hline${ }^{246}$ Es & ${ }^{249} \mathrm{Cf}(\mathrm{p}, 4 \mathrm{n}){ }^{246} \mathrm{Es}$ & 37 & $6 \pm 4 \mu b$ & $0.35 \mathrm{mb}$ & $76 \mu \mathrm{b}$ \\
\hline${ }^{246}$ Es & ${ }^{249} \mathrm{Cf}(\mathrm{p}, 4 \mathrm{n}){ }^{246} \mathrm{Es}$ & 40 & $3 \pm 2 \mu b$ & $0.11 \mathrm{mb}$ & $23 \mu \mathrm{b}$ \\
\hline${ }^{247}$ Es & ${ }^{249} \mathrm{Cf}(\mathrm{p}, 3 \mathrm{n}){ }^{247} \mathrm{Es}$ & $18 \pm 1.4$ & $11 \pm 9 \mu \mathrm{b}$ & $1.5 \mathrm{mb}$ & $1.6 \mu \mathrm{b}$ \\
\hline${ }^{247}$ Es & ${ }^{249} \mathrm{Cf}(\mathrm{p}, 3 \mathrm{n}){ }^{247} \mathrm{Es}$ & $21 \pm 1.4$ & $0.13 \pm 0.10 \mathrm{mb}$ & $3.7 \mathrm{mb}$ & $0.20 \mathrm{mb}$ \\
\hline${ }^{247}$ Es & ${ }^{249} \mathrm{Cf}(\mathrm{p}, 3 \mathrm{n}){ }^{247} \mathrm{Es}$ & 28 & $0.19 \pm 0.15 \mathrm{mb}$ & $1.9 \mathrm{mb}$ & $63 \mu b$ \\
\hline${ }^{247}$ Es & ${ }^{249} \mathrm{Cf}(\mathrm{p}, 3 \mathrm{n}){ }^{247} \mathrm{Es}$ & 35 & $12 \pm 10 \mu \mathrm{b}$ & $0.15 \mathrm{mb}$ & $6.4 \mu \mathrm{b}$ \\
\hline${ }^{247}$ Es & ${ }^{249} \mathrm{Cf}(\mathrm{p}, 3 \mathrm{n}){ }^{247} \mathrm{Es}$ & 37 & $11 \pm 7 \mu \mathrm{b}$ & $51 \mu b$ & $4.8 \mu \mathrm{b}$ \\
\hline${ }^{247}$ Es & ${ }^{249} \mathrm{Cf}(\mathrm{p}, 3 \mathrm{n}){ }^{247} \mathrm{Es}$ & 40 & $20 \pm 16 \mu b$ & $5.4 \mu \mathrm{b}$ & $5.9 \mu \mathrm{b}$ \\
\hline${ }^{248}$ Es & ${ }^{249} \mathrm{Cf}(\mathrm{p}, 2 \mathrm{n}){ }^{248} \mathrm{Es}$ & $18 \pm 1.4$ & $1.3 \pm 1.2 \mathrm{mb}$ & $19 \mathrm{mb}$ & $1.9 \mathrm{mb}$ \\
\hline${ }^{248}$ Es & ${ }^{249} \mathrm{Cf}(\mathrm{p}, 2 \mathrm{n}){ }^{248} \mathrm{Es}$ & $21 \pm 1.4$ & $1.9 \pm 0.6 \mathrm{mb}$ & $9.2 \mathrm{mb}$ & $0.56 \mathrm{mb}$ \\
\hline${ }^{248}$ Es & ${ }^{249} \mathrm{Cf}(\mathrm{p}, 2 \mathrm{n}){ }^{248} \mathrm{Es}$ & 28 & $0.94 \pm 0.75 \mathrm{mb}$ & $0.12 \mathrm{mb}$ & $0.17 \mathrm{mb}$ \\
\hline${ }^{248} \mathrm{Es}$ & ${ }^{249} \mathrm{Cf}(\mathrm{p}, 2 \mathrm{2n})^{248} \mathrm{Es}$ & 40 & $0.10 \pm 0.09 \mathrm{mb}$ & $0.04 \mu b$ & $63 \mu \mathrm{b}$ \\
\hline${ }^{249}$ Es & ${ }^{249} \mathrm{Cf}(\mathrm{p}, 1 \mathrm{ln}){ }^{249} \mathrm{Es}$ & $18 \pm 1.4$ & $0.84 \pm 0.67 \mathrm{mb}$ & $0.13 \mathrm{mb}$ & $2.5 \mathrm{mb}$ \\
\hline${ }^{249}$ Es & ${ }^{249} \mathrm{Cf}(\mathrm{p}, \mathrm{ln}){ }^{249} \mathrm{Es}$ & $21 \pm 1.4$ & $0.54 \pm 0.14 \mathrm{mb}$ & $34 \mu \mathrm{b}$ & $1.8 \mathrm{mb}$ \\
\hline${ }^{249}$ Es & ${ }^{249} \mathrm{Cf}(\mathrm{p}, 1 \mathrm{n}){ }^{249} \mathrm{Es}$ & 28 & $0.15 \pm 0.12 \mathrm{mb}$ & $6.7 \mathrm{nb}$ & $0.85 \mathrm{mb}$ \\
\hline
\end{tabular}

${ }^{2}$ Calculated assuming a $100 \%$ EC branch in ${ }^{242} \mathrm{Es}$, and thus represents a lower limit for the cross section.

${ }^{\mathrm{b}}$ The following fission barrier scaling factors were used with the ALICE code: 2.6 for the ${ }^{233} \mathrm{U}\left({ }^{14} \mathrm{~N}, \mathrm{xn}\right){ }^{247-\mathrm{x}}$ Es reactions, 2.5 for the ${ }^{233} \mathrm{U}\left({ }^{15} \mathrm{~N}, \mathrm{xn}\right){ }^{248-\mathrm{x}} \mathrm{Es}$ and ${ }^{237} \mathrm{~Np}\left({ }^{12} \mathrm{C}, \mathrm{xn}\right){ }^{249-\mathrm{x}} \mathrm{Es}$ reactions, and 2.4 for the ${ }^{249} \mathrm{Cf}(\mathrm{p}, \mathrm{xn})^{250-\mathrm{x}}$ Es reactions.

${ }^{c}$ Determined using a single target configuration (Figure 4.1.) The other reactions were determined using 19 targets in the LIM target chamber (Figure 4.2.)

${ }^{\mathrm{d}}$ These values represent upper limits only. 
and an efficiency of $5 \pm 4 \%$ was used for the $21 \mathrm{MeV}$ and $28 \mathrm{MeV}$ beam energies.

Chemical separation efficiencies were determined by comparing the amount of ${ }^{249} \mathrm{Cf}$ in the chemically processed sample to that of a single MG collection, correcting for the number of foils. All of the cross sections calculated during the excitation function experiment are presented in Table 7.2. Our results matched those previously reported by Hatsukawa et al. [HAT89] within error.

In reactions with heavy beams (such as ${ }^{14} \mathrm{~N}$ ), the SPIT code was able to predict the magnitude and energy of the peak of the excitation function with relative accuracy. The shapes of the predicted functions, especially at lower energies, were too steep when compared to actual data. Around the peak of the function, however, SPIT is usually correct to within a factor of five, and is a good method for estimating what beam energy should be used for producing a particular isotope in a reaction.

With light beams such as protons, SPIT does not do as good a job in predicting excitation functions. For the $3 n, 4 n$ and $5 n$ reactions, the shapes of the predicted functions are an almost perfect match to actual data. The magnitudes of the predicted cross sections, however, are orders of magnitude too high. The opposite trend was observed for the $1 n$ and $2 n$ reactions. In those cases, the shapes of the predicted functions were not even close to the actual data, but the predicted magnitudes were not too far off. One reason why SPIT may not have been able to predict the shapes of these functions was that the model the code uses does not take the preequilibrium emission of particles from the compound nucleus into account. If a factor was included in the code to account for this phenomenon, then it might do a better job in predicting the shapes of the $1 \mathrm{n}$ and $2 n$ functions. Overall, SPIT should be avoided when dealing with light projectile 
reactions, because one could never be sure whether the code was predicting the correct shape or the correct cross sections. The ALICE code seems to be a better choice for the prediction of light projectile excitation function reactions, but the user must know a priori which fission barrier scaling factor to include with the code. If the wrong scaling factor is used, then the predicted cross sections are several orders of magnitude off from the actual values. 


\section{Conclusions and Future Research}

\subsection{Conclusions}

The TKE values determined in the ECDF of ${ }^{242} \mathrm{Es}$ and ${ }^{244} \mathrm{Es}$ indicate that the excitation energy imparted to the fissioning nucleus from the initial EC-decay tends to wash out shell effects in the fission fragments. This in turn leads to more deformed fission fragments and an overall lower TKE than in cases of spontaneous fission. This trend has also been observed in previous ECDF studies.

The $P_{D F}$ values for ${ }^{242} \mathrm{Es},{ }^{244} \mathrm{Es},{ }^{246} \mathrm{Es}$ and ${ }^{248} \mathrm{Es}$ increase exponentially as the $\mathrm{Q}_{\mathrm{EC}}$ values of the nuclides increase. The equation for $\mathrm{P}_{\mathrm{DF}}$ given in Chapter 2 has an exponential dependence on the difference between fission barrier height and $\mathrm{Q}_{\mathrm{EC}}$. Since the $P_{D F}$ versus $Q_{E C}$ relationship shown in Figure 7.2 is monotonic, that implies that the fission barrier heights do not vary much from one nuclide to the other, and therefore the $\mathrm{Q}_{\mathrm{EC}}$ must be the dominant factor influencing the magnitude of the $\mathrm{P}_{\mathrm{DF}}$.

The LIM gas-jet transport system was found to have a very low yield when used with reactions involving light projectiles. This problem is exacerbated when low-energy projectiles are used. To improve the yield of the LIM system with low energy projectiles, improvements must be made to the design of the LIM chamber itself. For instance, one design was tried where instead of using one gas-jet that swept reaction products from all of the targets, each target had its own individual gas-jet stream flowing in front of it. Several small capillaries were bundled together and entered the LIM chamber from below the targets. The individual capillaries were then fanned out so that a separate capillary was positioned in front of each target. After collecting reaction products, the gas-jet 
streams from each capillary were collected at the top of the LIM chamber. In this configuration, each target was swept with a separate gas-jet. Using this new construction, the yield of the LIM system went up by approximately 39\%. This was not as large an increase as we had hoped for, but it demonstrates that the yield of the LIM system can potentially be improved with a new design.

Comparison of experimentally determined cross sections from the ${ }^{233} \mathrm{U}\left({ }^{14} \mathrm{~N}, \mathrm{xn}\right){ }^{247-\mathrm{x}} \mathrm{Es},{ }^{233} \mathrm{U}\left({ }^{15} \mathrm{~N}, \mathrm{xn}\right){ }^{248-\mathrm{x}} \mathrm{Es}$ and ${ }^{237} \mathrm{~Np}\left({ }^{12} \mathrm{C}, \mathrm{xn}\right){ }^{239-\mathrm{x}}$ Es reactions to predictions made by the SPIT code illustrate that SPIT is able to predict the magnitudes of these cross sections relatively well, usually within a factor of five. The shapes of the functions are predicted correctly in the region surrounding the peak energy, but this agreement breaks down at low energies.

Results from the ${ }^{249} \mathrm{Cf}(\mathrm{p}, \mathrm{xn})^{250-\mathrm{x}}$ Es excitation function experiment show that SPIT is unable to predict both the magnitudes and shapes of excitation functions involving light projectiles. It can predict one or the other with relative accuracy, but not both simultaneously. The shapes of the functions predicted for the $1 \mathrm{n}$ and $2 \mathrm{n}$ reactions are incorrect because the preequilibrium emission of particles from the compound nucleus is not taken into account by the SPIT code. If this type of correction were included in SPIT, the predicted excitation functions for light projectile reactions would probably agree better with experimental data.

The ALICE code seems to be a better choice for predicting cross sections for reactions involving light beams. This code includes a factor that takes preequilibrium decay into account. However, the user must enter the correct fission barrier scaling factor into the code, or else the magnitudes of the predicted excitation functions will be off by 
several orders of magnitude. If this term is not known prior to using ALICE, then the correct cross sections cannot be calculated by the code.

\subsection{Future Research}

The study of ECDF has permitted the investigation of fission properties of neutron deficient actinides that would be impossible otherwise. As one goes up in neutron number while maintaining the same $\mathrm{Z}$, the $\mathrm{Q}_{\mathrm{EC}}$ values decrease, as seen in Figure 7.2. ${ }^{250} \mathrm{Es}$ has a $\mathrm{QEC}$ of only $1.84 \mathrm{MeV}$ [MÖL95], making it an unlikely candidate for $E C D F$, as our systematics indicate that its $P_{D F}$ would be $10^{-7}$. Even though there may be no more einsteinium isotopes that undergo ECDF, there are other nuclides that have relatively large $\mathrm{Q}_{\mathrm{EC}}$ values, and therefore may have measurable delayed fission branches.

The next step for ECDF would be the investigation of mendelevium (Md, $\mathrm{Z}=101$ ) isotopes. ECDF has been reported in ${ }^{250} \mathrm{Md}$ [GAN80], and it is possible that other isotopes may undergo delayed fission as well. Looking at Figure 7.2, it appears that the $\mathrm{P}_{\mathrm{DF}}$ function may still be increasing with $\mathrm{Q}_{\mathrm{EC}}$. The study of ECDF must be pushed to nuclides with larger Q-values to determine whether this function is continuing to increase, or if it will level off to some maximum $P_{D F}$ value. The study of mendelevium isotopes would add more data points to Figure 7.2, and help determine the shape of the function at larger $\mathrm{QEC}_{\mathrm{EC}}$ values.

Additional measurements of the TKE values of ${ }^{242} \mathrm{Cf}$ and ${ }^{244} \mathrm{Cf}$ should also be made. Because of the low number of fission events detected for both nuclides, the statistical errors of their corresponding TKE values are rather large. If the counting statistics of these measurements were improved in future experiments, it would help to 
confirm the trend of lower TKE values in cases of ECDF seen in Figure 7.1. The large errors currently associated with the TKE values of ${ }^{242} \mathrm{Cf}$ and ${ }^{244} \mathrm{Cf}$ creates some uncertainty as to whether or not these nuclides are following the same trend that was observed in measurements of other nuclides.

Another experiment for the future would be the determination of the $\alpha-$ to ECbranching ratio in ${ }^{242}$ Es. ${ }^{242}$ Es is interesting because of its large $\mathrm{QEC}_{E C}$ and $\mathrm{P}_{\mathrm{DF}}$ values. The half-life has been determined [SHA99] and the $\alpha$-decay properties have been reported as well [NIN96]. If the EC-branch could be measured, the production cross section of the ${ }^{233} \mathrm{U}\left({ }^{14} \mathrm{~N}, 5 \mathrm{n}\right){ }^{242}$ Es reaction could be determined, as well as the $\log f t$ of the EC-transition. From our data we were only able to set limits on the $\alpha$ - and EC-branches (see section 6.1.1.2).

Because so few fission events were detected during the ${ }^{246}$ Es and ${ }^{248}$ Es ECDF experiments, it would be worth while to confirm their $\mathrm{P}_{\mathrm{DF}}$ values. One way to do that would be to use the $\mathrm{x}$-ray/fission coincidence measurement setup described in Chapter 5 . The number of $\mathrm{K} \mathrm{x}$-rays coming from the initial EC-decay could be compared to the total number of fission events detected. This was attempted previously, but the low yield of the LIM system meant that not enough einsteinium activity was produced, making the $\mathrm{x}$ rays impossible to detect over the large ${ }^{249} \mathrm{Cf}$ background. Two improvements to the experiment would be needed. First of all, the efficiency of the LIM system would need improving so that the overall yield was closer to $70 \%$. Either a new target chamber or a new gas-jet delivery system would have to be constructed so that the low recoil ranges of the compound nuclei will not decrease the yield of the system. Second, the einsteinium produced in the reaction would need to be separated from the californium background. 
This could be done with a cation exchange column using $\alpha$-hydroxyisobutyrate ( $\alpha$-HIB) as the eluting agent [CHO56, SMI56]. $\alpha$-HIB is a complexing agent that is sensitive to the differences in size between the actinides. The actinides are eluted in reverse order of their atomic numbers, so that einsteinium would be eluted before californium. By choosing the correct $\mathrm{pH}$, einsteinium could be removed from the column, leaving the californium behind. This would remove the background activity, allowing for the detection of the $\mathrm{x}$-rays.

Due to limited available time at the 88-Inch Cyclotron at LBNL, the excitation function experiments were only done over small energy ranges. 'Hopefully, these functions can be extended further in both directions. It would be especially interesting to look at higher projectile energies to see if preequilibrium decay of the compound nucleus occurs during all reactions. It was plainly evident in the $1 \mathrm{n}$ and $2 \mathrm{n}$ exit channels of the ${ }^{249} \mathrm{Cf}(\mathrm{p}, \mathrm{xn}){ }^{250-\mathrm{x}}$ Es reactions, and there was evidence of it in the $3 \mathrm{n}$ reaction as well. By extending all of the excitation functions, experimentalists will have more information about the shapes and magnitudes of the functions, and it may prove useful to someone who wishes to use one of these reactions in the future.

Finally, future investigations into the SPIT code, the ALICE code and other cross section codes should be made to evaluate their accuracy. A preequilibrium factor should be included in the SPIT code, which would make the shapes of the predicted excitation functions more accurate. Also, fission barrier scaling factors for the ALICE code should be determined for a variety of compound nuclei. To be certain; no one code is perfect for all situations, but perhaps a combination of different codes would sufficiently cover most reactions used for isotope production. 


\section{References}

[AUM74] D. C. Aumann and G. Müllen, Nucl. Instr. Meth. 115, 75 (1974).

[BAS74] R. Bass, Nucl. Phys. A 231, 45 (1974).

[BER69] É. E. Berlovich and Yu. P. Novikov, Dok. Akad. Nauk SSSR 185, 1025 (1969) [Sov. Phys. -Doklady 14, 349 (1969).]

[BLA75] M. Blann, Ann. Rev. Nucl. Part. Sci. 25, 123 (19750.

[BLA82] M. Blann and J. Bisplinghoff, Lawrence Livermore National Laboratory Report UCID-19614 (1982).

[BON74] J. P. Bondorf, M. I. Sobel and D. Sperber, Phys. Reports 15(2), 83 (1974).

[BRI80] H. C. Britt, E. Cheifetz, D. C. Hoffman, J. B. Wilhelmy, R. J. Dupzyk and R. W. Lougheed, Phys. Rev. C 21(2), 761 (1980).

[BUR57] E. M. Burbidge, G. R. Burbidge, W. A. Fowler and F. Hoyle, Rev. Mod. Phys. 29, 547 (1957).

[CHO56] G. R. Choppin and R. J. Silva, J. Inor. Nucl. Chem. 3, 153 (1956).

[DUK70] C. L. Duke, P. G. Hansen, O. B. Nielsen and G. Rudstam, Nucl. Phys. A 151,609 (1970).

[ESK70] P. Eskola, Lawrence Radiation Laboratory Nuclear Chemistry Annual Report UCRL-2-426 (1970).

[ESK73] P. Eskola, Phys. Rev. C 7(1), 280 (1973).

[ESK73A] P. Eskola, K. Eskola, M. Nurmia and A. Ghiorso, Phys. Fenn. 8, 357 (1973). 
[FIR96] R. B. Firestone and V. S. Shirley, Editors, Table of Isotopes, $8^{\text {th }}$ ed. (Wiley Interscience, New York, 1996).

[FRI81] G. Friedlander, J. W. Kennedy, E. S. Macias and J. M. Miller, Nuclear and Radiochemistry, $3^{\text {rd }}$ ed. (John Wiley and Sons, New York, 1981).

[GAN80] Y. P. Gangrskii, M. B. Miller, L. V. Mikhailov and I. F. Kharisov, Sov. J. Nucl. Phys. 31(2), 162 (1980).

[GHI54] A. Ghiorso, G. B. Rossi, B. G. Harvey and S. G. Thompson, Phys. Rev. 93, 257 (1954).

[GHI55] A. Ghiorso, S. G. Thompson, G. H. Higgins, G. T. Seaborg, M. H. Studier, P. R. Fields, S. M. Fried, H. Diamond, J. F. Mech, G. L. Pyle, J. R. Huizenga, A. Hirsch, W. M. Manning, C. I. Browne, H. L. Smith and R. W. Spence, Phys. Rev. 99, 1048 (1955).

[GIN62] J. E. Gindler, The Radiochemistry of Uranium, Subcommittee on Radiochemistry, National Academy of Sciences - National Research Council (1962).

[GRE91] K. E. Gregorich, Nucl. Instr. Meth. A 302, 135 (1991).

[GRE96] K. E. Gregorich, private communication (1996).

[GRE96A] K. E. Gregorich, private communication (1996).

[GRE98] K. E. Gregorich, private communication (1998).

[HAB78] D. Habs, H. Klewe-Nebenius, V. Metag, B. Neumann and H. J. Specht, Z. Physik A 285, 53 (1978).

[HAL89] H. L. Hall, Ph.D. Thesis, Lawrence Berkeley Laboratory Report LBL27878 (1989). 
[HAL89A] H. L. Hall, M. J. Nurmia and D. C. Hoffman, Nucl. Instr. Meth. A 276, 649 (1989).

[HAL89B] H. L. Hall, K. E. Gregorich, R. A. Henderson, C. M. Gannett, R. B.

Chadwick, J. D. Leyba, K. R. Czerwinski, B. Kadkhodayan, S. A. Kreek, D. M. Lee, M. J. Nurmia and D. C. Hoffman, Phys. Rev. Lett. 63, 2548 (1989).

[HAL89C] H. L. Hall, K. E. Gregorich, R. A. Henderson, D. M. Lee, D. C. Hoffman, M. E. Bunker, M. M. Fowler, P. Lysaght, J. W. Starner and J. B. Wilhelmy, Phys. Rev. C 39, 1866 (1989).

[HAL90] H. L. Hall, K. E. Gregorich, N. J. Hannink, R. A. Henderson, C. M. Gannett, R. B. Chadwick, J. D. Leyba, K. R. Czerwinski, B. Kadkhodayan, S. A. Kreek, D. M. Lee, M. J. Nurmia, D. C. Hoffman, C. E. A. Palmer and P. A. Baisden, Phys. Rev. C 42, 618 (1990).

[HAL90A] H. L. Hall, K. E. Gregorich, R. A. Henderson, C. M. Gannett, R. B. Chadwick, J. D. Leyba, K. R. Czerwinski, B. Kadkhodayan, S. A. Kreek, D. M. Lee, M. J. Nurmia, D. C. Hoffman, C. E. A. Palmer and P. A. Baisden, Phys. Rev. C 41, 1480 (1990).

[HAT89] Y. Hatsukawa, T. Ontsuki, K. Sueki, H. Nakahara, I. Kohno, M. Magara, N. Shinohara, H. L. Hall, R. A. Henderson, C. M. Gannett, J. D. Leyba, R. B. Chadwick, K. E. Gregorich, D. M. Lee, M. J. Nurmia and D. C. Hoffman, Nucl. Phys. A 500, 90 (1989).

[HEN90] R. A. Henderson, Ph.D. Thesis, Lawrence Berkeley Laboratory Report LBL-29568 (1990). 
[HIG60] G. H. Higgins, The Radiochemistry of the Transcurium Elements, Subcommittee on Radiochemistry, National Academy of Sciences National Research Council (1960).

[HIL53] D. L. Hill and J. A. Wheeler, Phys. Rev. 89, 1102 (1953).

[HIN85] R. Hingmann, W. Kühn, V. Metag, R. Novotny, A. Ruckelshausen, H. Ströher, F. Heßberger, S. Hofmann, G. Münzenberg and W. Reisdorf, Gesellschaft für Schwerionenforschung, Darmstadt, Report No. GSI 85-1, 88 (1985).

[HOD67] P. E. Hodgson, Ann. Rev. Nucl. Sci. 17,1 (1967).

[HOF94] S. Hofmann, V. Ninov, F. P. Heßberger, H. Folger, G. Münzenberg, H. J. Schött, P. Armbruster, A. N. Andreyev, A. G. Popeko, A. V. Yeremin, M. E. Leino, R. Janik, S. Saro and M. Veselsky, Gesellschaft für Schwerionenforschung, Darmstadt, Report No. GSI 94-1 (1994).

[HOFF86] R. W. Hoff in Weak and Electromagnetic Interactions in Nuclei, H. V. Klapdor, Editor (Springer-Verlag, Heidelberg, 1986).

[HOFF88] R. W. Hoff, Inst. Phys. Conf. Ser. No. 88; J. Phys. G: Nucl. Phys. 14 Suppl., S343 (1986).

[HOFFM74] D. C. Hoffman and M. M. Hoffman, Annu. Rev. Nucl. Sci. 24, 151 (1974).

[HOFFM89] D. C. Hoffman and L. P. Somerville in Charged Particle Emission from Nuclei, D. N. Poenaru and M. Ivascu, Editors (Chemical Rubber Company, Florida, 1989), Vol. III. 
[HOFFM90] D. C. Hoffman, D. M. Lee, K. E. Gregorich, M. J. Nurmia, R. B.

Chadwick, K. B. Chen, K. R. Czerwinski, C. M. Gannett, H. L. hall, R.

Henderson, B. Kadkhodayan, S. A. Kreek and J. D. Leyba, Phys. Rev. C 41, $631(1990)$.

[HOR75] P. Hornshøj, B. R. Erdal, P. G. Hansen, B. Jonson, K. Aleklett and G. Nyman, Nucl. Phys. A 239, 15 (1975).

[ITK88] M. G. Itkis, V. N. Okolvich, A. Yu. Rusanov and G. N. Smirenkin, Sov. J. Part. Nucl. 19(4), 301 (1988).

[JEN58] R. C. Jensen and A. W. Fairhall, Phys. Rev. 109, 942 (1958).

[KLA79] H. V. Klapdor, C. -O. Wene, I. N. Isosimov and Yu. W. Naumow, Z. Physik A 292, 249 (1979).

[KLA81] H. V. Klapdor, T. Oda, J. Metzinger, W. Hillebrandt and F. K. Thielemann, Z. Physik A 299, 213 (1981).

[KOD75] T. Kodama and K. Takahashi, Nucl. Phys. A 239, 489 (1975).

[KON69] E. Konecny, W. Nörenberg and H. W. Schmitt, Nucl. Phys. A 139, 513 (1969).

[KRA73] K. -L. Kratz and G. Herrmann, Z. Physik 263, 435 (1973).

[KRA76] K. -L. Kratz, W. Rudolph, H. Ohm, H. Franz, G. Herrmann, C. Ristori, J. Crancon, M. Asghar, G. I. Crawford, F. M. Nun and S. G. Prussin, Phys. Lett. 65B, 231 (1976).

[KRA79] K. -L. Kratz, W. Rudolph, H. Ohm, H. Franz, M. Zendel, G. Herrmann, S. G. Prussin, F. M. Nun, A. A. Shihab-Eldin, D. R. Slaughter, W. Halverson and H. V. Klapdor, Nucl. Phys. A 317, 335 (1979). 
[KRA88] K. S. Krane, Introductory Nuclear Physics (John Wiley and Sons, New York, 1988).

[KRE93] S. A. Kreek, Ph.D. Thesis, Lawrence Berkeley Laboratory Report LBL33766 (1993).

[KRE94] S. A. Kreek, H. L. Hall, K. E. Gregorich, R. A. Henderson, J. D. Leyba, K. R. Czerwinski, B. Kadkhodayan, M. P. Neu, C. D. Kacher, T. M. Hamilton, M. R. Lane, E. R. Sylwester, A. Türler, D. M. Lee, M. J. Nurmia and D. C. Hoffman, Phys. Rev. C 49, 1859 (1994).

[KRE94A] S. A. Kreek, H. L. Hall, K. E. Gregorich, R. A. Henderson, J. D. Leyba, K. R. Czerwinski, B. Kadkhodayan, M. P. Neu, C. D. Kacher, T. M. Hamilton, M. R. Lane, E. R. Sylwester, A. Türler, D. M. Lee, M. J. Nurmia and D. C. Hoffman, Phys. Rev. C 50, 2288 (1994).

[KUZ66] V. I. Kuznetsov, N. K. Skobelev and G. N. Flerov, Yad. Fiz. 4, 279 (1966) [Sov. J. Nucl. Phys. 4, 202 (1967)]

[KUZ67] V. I. Kuznetsov, N. K. Skobelev and G. N. Flerov, Yad. Fiz. 5, 271 (1967) [Sov. J. Nucl. Phys. 5, 191 (1967)]

[LAN98] M. R. Lane, K. E. Gregorich, D. M. Lee, B. Wierczinski, C. A. McGrath, M. B. Hendricks, D. A. Shaughnessy, D. A. Strellis, E. R. Sylwester, P. A. Wilk and D. C. Hoffman, Phys. Rev. C 58, 3413 (1998).

[LAU98] C. A. Laue, private communication (1998).

[LEE96] D. M. Lee, private communication (1996).

[LEY90] J. D. Leyba, Ph.D. Thesis, Lawrence Berkeley Laboratory Report LBL29540 (1990). 
[MEY86] B. S. Meyer and D. N. Schramm, Astrophys. J. 311, 406 (1986).

[MEY89] B. S. Meyer, W. M. Howard, G. J. Matthews, K. Takahashi, P. Möller and G. Leander, Phys. Rev. C 39, 1876 (1989).

[MIK67] V. L. Mikheev, V. I. Ilyushchenko and M. B. Miller, Sov. J. Nucl. Phys. 5(1), 35 (1967).

[MÖL95] P. Möller, J. R. Nix and W. J. Swiatecki, Atomic Data Nucl. Data Tables 59, 185 (1995).

[MÜL75] G. Müllen and D. C. Aumann, Nucl. Instr. Meth. 128, 425 (1975).

[NIN96] V. Ninov, F. P. Heßberger, S. Hofmann, H. Folger, G. Münzenberg, P. Armbruster, A. V. Yeremin, A. G. Popeko, M. Leino and S. Saro, Z. Physik A 356(1), 11 (1996).

[NOR70] L. C. Northcliffe and R. F. Schilling, Nucl. Data Tables A7, 233 (1970).

[NUR67] M. J. Nurmia, T. Sikkeland, R. Silva and A. Ghiorso, Phys. Lett. B26, 78 (1967).

[PAP72] A. C. Pappas and T. Sverdrup, Nucl. Phys. A 188, 48 (1972).

[PEP57] D. F. Peppard, S. W. Moline and G. W. Masm, J. Inorg. Nucl. Chem. 4, 344 (1957).

[POE89] D. N. Poenaru, M. S. Ivaşcu and D. Mazilu in Charged Particle Emission from Nuclei, D. N. Poenaru and M. Ivaşcu, Editors (Chemical Rubber Company, Florida, 1989), Vol. III.

[POS61] A. M. Poskanzer and B. M. Foreman Jr., J. Inorg. Nucl. Chem. 16, 323 (1961). 
[RAT91] W. Rathbun, Lawrence Berkeley National Laboratory Report LBL-29734 (1991).

[SCH65] H. W. Schmitt, W. E. Kiker and C. W. Williams, Phys. Rev. 137, B837 (1965).

[SEA90] G. T. Seaborg and W. D. Loveland, The Elements Beyond Uranium (John Wiley and Sons, New York, 1990).

[SHA77] S. Shalev and G. Rudstam, Nucl. Phys. A 275, 76 (1977).

[SHA99] D. A. Shaughnessy, J. L. Adams, K. E. Gregorich, M. R. Lane, C. A. Laue, D. M. Lee, C. A. McGrath, J. B. Patin, D. A. Strellis, E. R. Sylwester, P. A. Wilk and D. C. Hoffman, Phys. Rev. C (in press, 1999).

[SIK67] T. Sikkeland, Ark Fys. 36, 539 (1967); J. Alonso in Gmelin Handbook of Inorganic Chemistry, R. Warncke, Editor (Springer-Verlag, New York, 1974), Vol. 7b, p. 28.

[SIK68] T. Sikkeland, J. Maly and D. F. Lebeck, Phys. Rev. 169, 1000 (1968).

[SIK68A] T. Sikkeland, A. Ghiorso and M. J. Nurmia, Phys. Rev. 172, 1232 (1968).

[SKO72] N. K. Skobelev, Sov. J. Nucl. Phys. 15(3), 249 (1972).

[SMI56] H. L. Smith and D. C. Hoffman, J. Inor. Nucl. Chem. 3, 243 (1956).

[STA92] A. Staudt and H. V. Klapdor-Kleingrothaus, Nucl. Phys. A 549, 254 (1992).

[STR99] D. A. Strellis, private communication (1999).

[SWI83] W. J. Swiatecki, Aust. J. Phys. 36, 641 (1983).

[THE83] F. -K. Theilemann, J. Metzinger and H. V. Klapdor, Astron. and Astrophys. 123, 162 (1983). 
[THO68] T. D. Thomas, Ann. Rev. Nucl. Sci. 18, 343 (1968).

[UNI73] J. P. Unik, J. E. Gindler, L. E. Glendenin, K. F. Flynn, A. Gorski and R. K. Sjoblom, Proceedings of the Third International IAEA Symposium on the Physics and Chemistry of Fission, Rochester, 1973 (IAEA, Vienna, 1974), Vol. II, p. 19.

[VAN73] R. Vandenbosch and J. R. Huizenga, Nuclear Fission (Academic Press, New York, 1973).

[VIO85] V. E. Viola, K. Kwiatkowski and M. Walker, Phys. Rev. C 31, 1550 (1985).

[WEI86] E. Weissenberger, P. Geltenbort, A. Oed, F. Gönnenwein and H. Faust, Nucl. Instr. Meth. A 248, 506 (1986).

[WEN74] C. -O. Wene and S. A. E. Johansson, Phys. Scripta 10A, 156 (1974).

[WEN75] C. -O. Wene, Astron. and Astrophys. 44, 233 (1975).

[WIL76] B. D. Wilkins, E. P. Steinberg and R. R. Chasman, Phys. Rev. C 14(5), 1832 (1976).

[WIL97] P. A. Wilk, K. E. Gregorich, M. B. Hendricks, M. R. Lane, D. M. Lee, C. A. McGrath, D. A. Shaughnessy, D. A. Strellis, E. R. Sylwester and D. C. Hoffman, Phys. Rev. C 56, 1626 (1997).

[YOK83] K. Yokoi, K. Takahashi and M. Arnould, Astron. and Astrophys. 117, 65 (1983). 